Effector-mediated microbiome manjipulation by the soil-borne fungal plant pathogen Verticilliums dafliljae

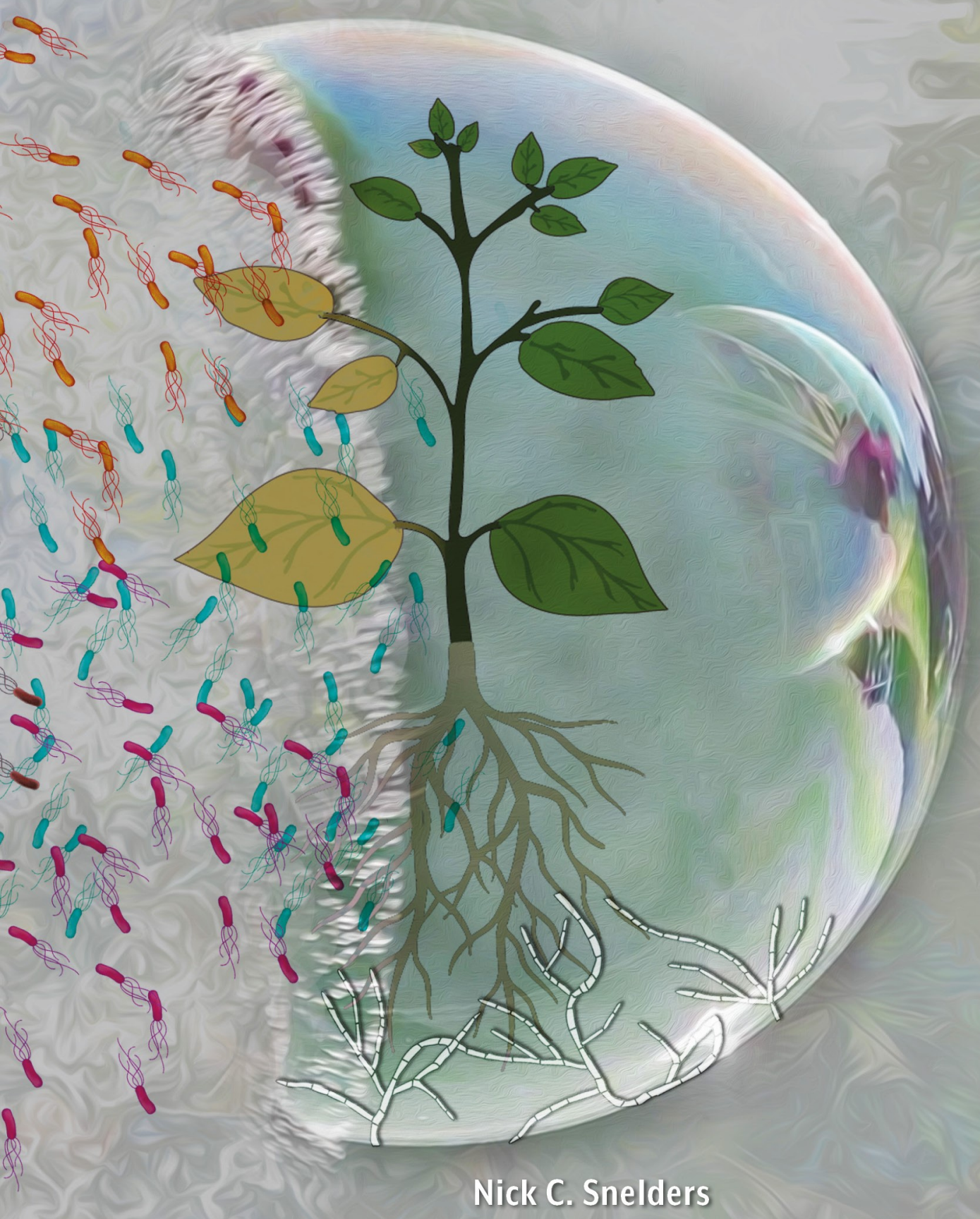




\section{Propositions}

1. Plant pathogens utilize effector proteins to manipulate host microbiomes (this thesis)

2. Plant natriuretic peptides are lysozymes (this thesis)

3. Probiotic synthetic communities are the performance-enhancing drugs of the future for professional athletes

4. The use of patient-derived kidney organoids for initial drug screening will spur the design of personalized therapies to treat kidney disease

5. The fastest way for a wet-lab scientist to positively impact our planet is to cut the use of plastic disposables in the lab

6. For cycling scientists, Hirsch-indices should remain subordinate to Eddington numbers

7. In our increasingly polarized world, provocative society-related propositions have become obsolete

Propositions belonging to the thesis, entitled

Effector-mediated microbiome manipulation by the soil-borne fungal plant pathogen Verticillium dahliae

Nick C. Snelders

Wageningen, 4 December 2020 
Effector-mediated microbiome manipulation by the soil-borne fungal plant pathogen Verticillium dahliae

Nick C. Snelders 


\section{Thesis committee}

\section{Promotor}

Prof. Dr B.P.H.J. Thomma

Professor of Phytopathology

Wageningen University \& Research

\section{Other members}

Prof. Dr J. van der Oost, Wageningen University and Research

Prof. Dr L. Mommer, Wageningen University and Research

Prof. Dr C.M.J. Pieterse, Utrecht University

Prof. Dr E.H. Stukenbrock, Christian-Albrechts University of Kiel, Germany

This research was conducted under the auspices of the Graduate School Experimental Plant Sciences. 


\title{
Effector-mediated microbiome manipulation by the soil-borne fungal plant pathogen Verticillium dahliae
}

\author{
Nick C. Snelders
}

Thesis

submitted in fulfilment of the requirements for the degree of doctor at Wageningen University

by the authority of the Rector Magnificus,

Prof. Dr A.P.J. Mol,

in the presence of the

Thesis Committee appointed by the Academic Board

to be defended in public

on Friday 4 December 2020

at 1:30 p.m. in the Aula. 
Nick C. Snelders

Effector-mediated microbiome manipulation by the soil-borne fungal plant pathogen Verticillium dahliae, 174 pages.

PhD thesis, Wageningen University, Wageningen, the Netherlands (2020)

With references, with summary in English

ISBN : 978-94-6395-556-0

DOI: https://doi.org/10.18174/531764 


\section{Table of contents}

$\begin{array}{lll}\text { Chapter } 1 \text { General introduction } & 7\end{array}$

Chapter 2 Plant pathogen effector proteins as manipulators of host

microbiomes?

Chapter 3 Microbiome manipulation by a soil-borne fungal plant pathogen using effector proteins

Chapter 4 Teichoic acids protect Bacillus subtilis against the antimicrobial Verticillium dahliae effector VdAve1

Chapter 5 Functional characterization uncovers the antimicrobial Verticillium dahliae effector VdAve1 as a novel type of lysozyme

Chapter 6 A Verticillium dahliae defensin-like effector protein promotes microsclerotia formation and survival after host decay

Chapter 7 General discussion

References

Summary

Acknowledgements

About the author

List of publications 



\section{Chapter 1}

\section{General introduction}

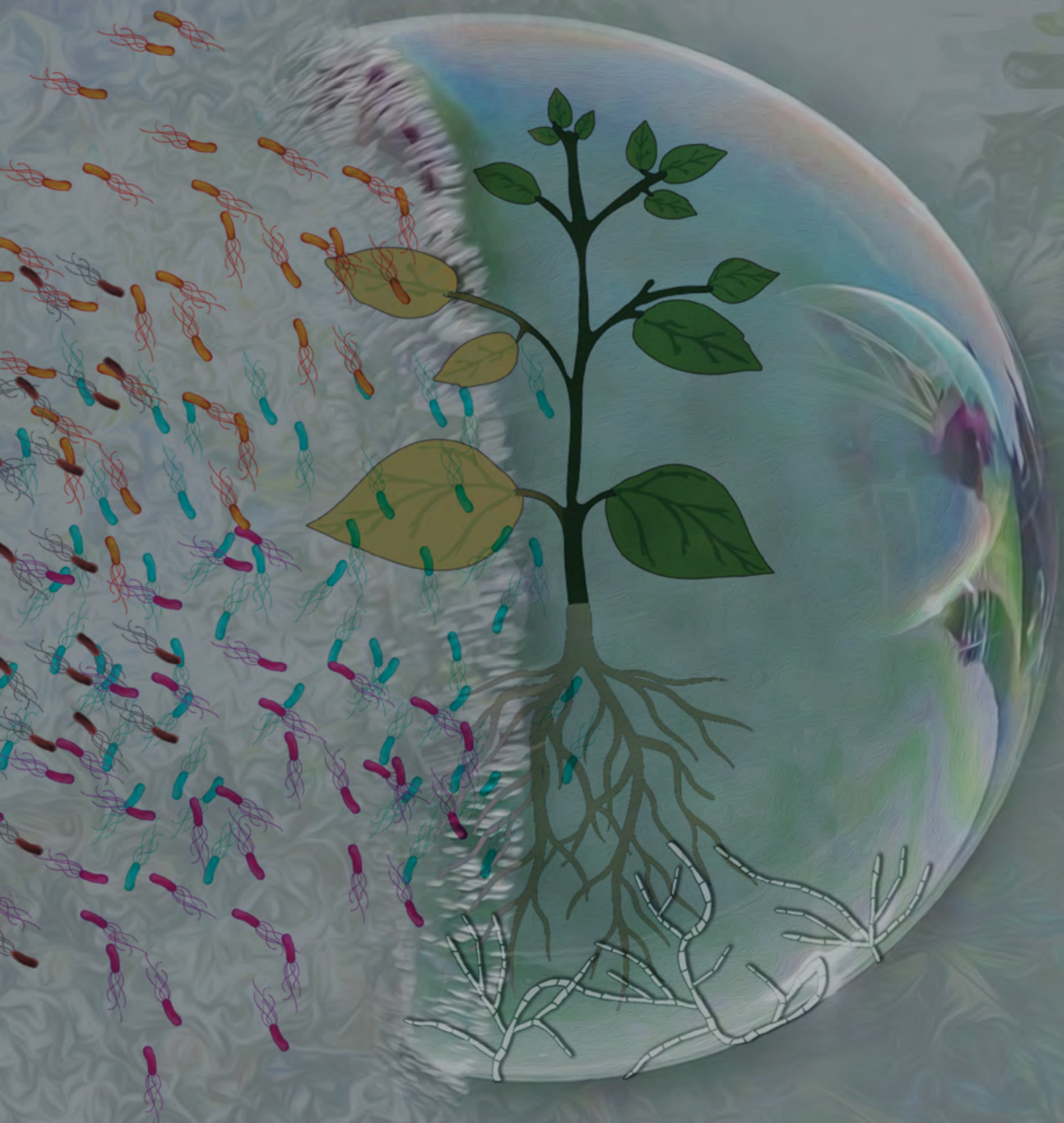




\section{The exogenous and endogenous layers of the plant immune system}

The totality of microbes a plant associates with, its so-called microbiota, encompasses a diverse group of microbes that establish a broad spectrum of symbiotic relationships with their host, ranging from commensalistic through endophytic to mutualistic and pathogenic. To survey their microbiota for the presence of potentially pathogenic invaders, plants have evolved complex immune systems comprising various types of receptors that betray pathogen invasion and induce immune responses ${ }^{1}$. Since nearly 15 years, the interaction between plant pathogens and their hosts is often described using the so-called "zigzag" model2. This model postulates that plants utilize cell surface-localized pattern recognition receptors (PRRs) to detect general elicitors, socalled microbe-associated molecular patterns (MAMPs), in order to mount a broadspectrum immune response that is also known as MAMP-triggered immunity (MTI). The essential addition of the zigzag model over previous models is the notion that successful plant pathogens evolved to secrete "effector" proteins that suppress MTI, leading to effector-triggered susceptibility (ETS). The model furthermore states that, in turn, plants evolved to recognize such effectors, or their activities, through cytoplasmic resistance (R) proteins that activate effector-triggered immunity (ETI) to restrict pathogen invasion. Plant pathogens can overcome ETI again through loss or mutation of the effector proteins that have become recognized, or through active suppression of the ETI response using novel effectors. These processes describe the continued molecular arms race between plant pathogens and their hosts.

Following its introduction, continuing research in the field of plant-microbe interactions has gradually unveiled several limitations of the zigzag model ${ }^{3,4}$. Consequently, the "Invasion Model" was drafted to account for discrepancies of the zigzag model, such as the strict separation of MTI and ETI in time and space, as well as the paradigm that MAMPs and effectors should be considered mutually exclusive entities ${ }^{5}$. Instead, the Invasion Model states that plants exploit various types of receptors, collectively termed invasion pattern receptors (IPRs), to detect any type of molecule that reliably betrays microbial ingress, either microbial-derived or modified-self, collectively termed invasion patterns (IPs), to induce the most appropriate immune responses. The Invasion Model is applicable to a wide variety of symbiotic microbes, including non-pathogenic endophytes and mutualists that either utilize or suppress IP-triggered responses (IPTR) to establish symbioses.

Plant microbiota comprise a wide variety of microbes. These microbes, together with their genomes and the environment are typically referred to as the so-called plant microbiome ${ }^{6}$. Importantly, the plant microbiome is a key determinant for plant health. Above-ground as well as below-ground plant organs are colonized by beneficial microbes that are able to alleviate (a)biotic stresses ${ }^{7-11}$. Especially the role of the root microbiome in plant health has been well described ${ }^{10}$. The microbial communities 
in the soil represent an enormous pool of biological diversity, and although soil is considered to be microbe-rich, microbial proliferation is still limited by the relatively poor nutrient availability in bulk soil ${ }^{12}$. Plants release a significant amount of their photosynthetically fixed carbon into the rhizosphere, the narrow zone of soil in close proximity to their roots. These nutrient-rich root exudates attract microbes, which leads to an increased microbial density in the rhizosphere when compared with the bulk soil ${ }^{13,14}$. This phenomenon, known as the rhizosphere effect, illustrates the intimate relationship between plants and their associated root microbiota ${ }^{10}$. Plants are able to define the composition of their root microbiome and to specifically stimulate the growth of beneficial microbes through the secretion of root exudates ${ }^{10,15-17}$. The microbes that are selected for in this manner help plants to mitigate abiotic stresses, such as drought, nutrient deficiencies and toxic compounds $9,11,18$. Moreover, beneficial root-associated microbes play important roles in the suppression of microbial plant pathogens. Importantly, plants exploit root exudates to actively recruit beneficial microbes upon pathogen attack to suppress disease ${ }^{10,15,19-21}$. This disease suppression often involves direct antibiosis through the secretion of various types of molecules, including hydrolytic enzymes, antimicrobial proteins and secondary metabolites, but beneficial rhizobacteria can also indirectly affect above-ground as well as belowground plant pathogens through the induction of systemic immune responses ${ }^{22,23}$. Consequently, beneficial microbiomes can be seen as an inherent, exogenous, layer of defense that complements the endogenous plant innate immune system to restrict pathogen invasion ${ }^{24}$.

\section{Plant pathogen effector molecules}

As recognized in the zigzag model described above, plant pathogens have evolved various mechanisms to overcome the innate immune systems of their hosts, which typically involve the secretion of effector molecules ${ }^{25,26}$. Initially, the term effector was exclusively assigned to pathogen-encoded small cysteine-rich in planta-secreted proteins that were thought to be involved in the suppression of immune responses. Ongoing research, however, revealed that non-proteinaceous molecules, such as secondary metabolites and small RNAs, can fulfil typical effector functions during host colonization as well, and therefore should also be considered part of microbial effector repertoires ${ }^{27,28}$. Additionally, it has become clear that effector molecules exert many other activities that, for instance, also involve self-protection from host-secreted antimicrobial compounds, manipulation of host metabolism to liberate nutrients, or perturbation of pathogen recognition by the host ${ }^{29,30}$. Importantly, whereas effectors were initially considered to be pathogen-specific molecules, it is increasingly recognized that effector molecules are not exclusively secreted by plant pathogens to promote host colonization, but also by other types of plant-associated microbes such 
as endophytes and mutualists that were originally hypothesized not to trigger host immune responses ${ }^{31-33}$.

Lysin motif (LysM) effectors represent a well-studied family of secreted chitin-binding proteins that are widely distributed in the fungal kingdom ${ }^{34,35}$. Accordingly, LysM effectors have been identified in numerous plant-associated fungi with lifestyles ranging from pathogenic to mutualistic ${ }^{34}$. For many of these fungi, LysM effectors fulfill crucial roles in the deregulation of immune responses and colonization of their hosts ${ }^{30,32,36,37}$. Importantly, however, LysM effectors are not confined to plantassociated fungi, as numerous genes encoding LysM effectors have been identified in the genomes of free-living saprophytic fungi and (opportunistic) animal pathogens ${ }^{34}$. An even more broadly distributed group of effector proteins is the family of the Necrosis- and ethylene-inducing-like proteins (NLPs) secreted by bacteria, fungi and oomycetes ${ }^{38-40}$. Thus far, NLPs have predominantly been characterized as phytotoxic virulence factors encoded by plant pathogenic microbes. However, it is important to note that not all NLPs secreted by plant pathogens exert phytotoxic activity, and that NLPs also occur in non-pathogenic species ${ }^{40}$.

The existence of effector protein families that are conserved across pathogenic and non-pathogenic microbes inhabiting a diversity of niches suggests that effectors are broadly applied, and hints towards effector functions beyond host-microbe interactions. Like free-living saprophytes that thrive on decaying organic matter in the soil, many plant pathogens also undergo saprophytic life stages outside their hosts as part of their life cycle. Environments in which such saprotrophism occurs are generally characterized by rich microbiota, meaning that the saprotroph is exposed to a wealth of microbes. Consequently, pathogenic as well as non-pathogenic microbes can be anticipated to secrete molecules that help to establish themselves in competitive microbial communities. Accordingly, it has previously been hypothesized that microbes exploit effector molecules to shape the composition of local microbiomes by influencing co-inhabiting microbes, either positively or negatively, to facilitate niche colonization ${ }^{25}$. Building on this hypothesis, in my thesis, effectors are provocatively defined as microbially secreted molecules that act in niche colonization.

Considering the positive impact of plant-associated microbial communities on plant health, host colonization by pathogenic microbes likely does not exclusively result from successful host manipulation. Rather, disease establishment by a plant pathogen is more likely to be the outcome of successful interactions with its host as well as the associated microbiota, collectively termed the plant holobiont. It is relevant to realize, however, that pathogen-secreted effectors are almost exclusively studied in the context of binary plant-pathogen interactions, while their effects on other microbes in the context of the plant holobiont have largely been overlooked or ignored. Since plants assemble beneficial microbiomes that serve as an exogenous layer of the plant immune system, microbial plant pathogens can be 
anticipated to target these microbiomes to compromise plant health and establish disease. Arguably, secreted effector molecules can represent excellent tools for such microbiome manipulation. Consequently, I hypothesize that microbial plant pathogens exploit effector proteins to promote disease establishment through the manipulation of host microbiomes.

Thus far, the role of plant pathogen effector proteins in manipulation of hostassociated microbial communities, as well as the microbiota encountered during free-living life stages outside the host, has remained largely unexplored. However, particular pathogen-secreted effector proteins have been found to display antimicrobial activity in vitro, although the actual contribution of those proteins to host colonization through enhanced microbial competition has remained undemonstrated ${ }^{41,42}$. For instance, the ribonuclease effector $Z t 6$, secreted by the wheat pathogen Zymoseptoria tritici, was shown to display both phytotoxic as well as antimicrobial activity when applied exogenously to plant leaves and microbes, respectively ${ }^{42}$. However, targeted deletion of Zt6 did not result in reduced wheat colonization, and particularly the biological significance of the microbial growth inhibition has remained unclear. In addition to proteins that might offensively target microbial co-inhabitants, pathogen effectors may also act in self-defense against antimicrobial compounds secreted by microbial competitors. Along these lines, it was previously hypothesized that pathogenic fungi not only secrete their chitin-binding LysM effector proteins to shield their hyphae from hydrolytic enzymes released by plants, but also from such enzymes that are secreted by mycoparasites ${ }^{43}$. Nevertheless, experimental evidence for this hypothesis, as well as the direct involvement of any other plant pathogen effector protein in microbial interactions, is still lacking.

\section{Study system: the soil-borne fungal pathogen Verticillium dahliae}

The Ascomycete fungal genus Verticillium consists of ten soil-borne species that are presumed to be asexual and that are engaged in a range of lifestyles ${ }^{44}$. $V$. tricorpus, $V$. zaregamsianum, V. nubilum, V. isaacii and V. klebahnii are considered to be free-living saprophytes that incidentally cause disease as opportunistic pathogens on weakened plants, but typically thrive on decaying organic matter in the soil44-46. The remaining species, V. dahliae, V. longisporum, V. albo-atrum, V. alfalfae and V. nonalfalfae, are xylem-invading plant pathogens ${ }^{47-51} . V$. dahliae is the most notorious plant pathogen of the Verticillium genus as it causes disease on hundreds of plant species ${ }^{52}$. Important $V$. dahliae hosts, amongst many others, include the solanaceous crops tomato, potato, bell pepper and eggplant, the cucurbit crops watermelon and cucumber, but also other crops such as sugar beet, sunflower, cotton and even woody species including olive, apricot and cocoa. The other pathogenic Verticillium species have considerably 
narrower host ranges. $V$. longisporum generally infects brassicaceous plants and is infamous for its ability to cause Verticillium stem striping on oilseed rape ${ }^{49}$. V. alboatrum and $V$. nonalfalfae cause Verticillium wilt in potato and hop, respectively, while $V$. alfalfae so far has only been reported to infect alfalfa ${ }^{50,52}$.

Verticillium spp. survive in the soil through persistent melanized resting structures, including microsclerotia, resting mycelium and chlamydospores, that offer protection against (a)biotic stresses and survive in the soil for many years ${ }^{48,53} . V$. dahliae forms microsclerotia, which represent the primary inoculum source in nature. $V$. dahliae microsclerotia germinate upon stimulation by carbon- and nitrogen-rich exudates released by plant roots in close proximity to the fungus ${ }^{54,55}$. Following subsequent saprophytic growth, V. dahliae penetrates the roots, grows through the cortex, and enters the xylem vessels of its hosts where condiospores are produced (Fig 1.). These conidiospores are transported to distal tissues with the sap stream and germinate once they get trapped ${ }^{48}$. Upon germination, the fungus can penetrate cell walls, enter new xylem vessels, and sporulate again. This systemic colonization leads to chlorosis, necrosis and wilting of the host. During plant senescence, V. dahliae enters a saprophytic stage during which it emerges from the xylem vessels to colonize decaying host tissues where it produces new microsclerotia that are released into the soil upon tissue decomposition ${ }^{48}$.

Due to its broad host range and the production of persistent microsclerotia, $V$. dahliae is difficult to control by means of crop rotation ${ }^{56}$. Moreover, since a significant part of the life cycle takes place in the vascular system, the possibilities for disease control using chemicals are limited as fungicides generally cannot affect the fungus once in the xylem ${ }^{48}$. Furthermore, only few genetic resistance sources to Verticillium wilt disease have been identified, which greatly limits the possibilities to constrain $V$. dahliae through resistance breeding. In tomato, the Ve locus was described to provide resistance against $V$. dahliae and has been introgressed into most commercial tomato cultivars since the $1950 \mathrm{~s}^{57,58}$. The Ve locus contains two closely linked genes, Ve1 and $V e 2$, that both encode extracellular leucine rich repeat receptor-like proteins (eLRRRLPs), of which only Ve1 was identified to confer $V$. dahliae resistance ${ }^{59}$. However, shortly after its deployment, resistance-breaking $V$. dahliae isolates appeared that were assigned to race 2 of the pathogen, while isolates that were contained by the Ve locus were assigned to race $1^{60}$.

Through comparative population genomics, the effector gene Ave1 (Avirulence on Ve1 tomato) was identified within the highly dynamic lineage-specific (LS) regions of $V$. dahliae race 1 strains, and was uncovered as the gene encoding the invasion pattern that is responsible for activating Ve1-mediated resistance ${ }^{61} . V$. dahliae LS regions are enriched for in planta-induced effector genes, several of which have been shown to promote host colonization ${ }^{36,62}$. Accordingly, functional analyses revealed that VdAve1 acts as a major virulence factor on plants lacking the Ve1 immune receptor ${ }^{61}$. 
So far, functional Ve1 homologs have only been described for a small number of $V$. dahliae host plants besides tomato ${ }^{63}$. Moreover, the introduction of resistant tomato varieties carrying Ve 1 was rapidly succeeded by an increased incidence of Verticillium wilt caused by resistance-breaking race 2 strains that lack Ave 160,64. Consequently, Ve1 does not provide a broadly-applicable solution to Verticillium wilt diseases. Only recently, decades after the description of the first $V$. dahliae race 2 strains, a single dominant locus, denoted $V 2$, was identified to mediate race 2 resistance ${ }^{65}$. Unfortunately, however, pathogenicity assays conducted using race 2-resistant rootstocks immediately resulted in the identification of resistance-breaking $V$. dahliae isolates, that are now designated as race $3^{65}$. Recently, comparative genomics between race 2 and race 3 strains uncovered an effector gene located in a race 2 -specific sequence as the gene encoding the effector protein responsible for the V2mediated resistance, which was termed $\mathrm{Av}^{66}$.

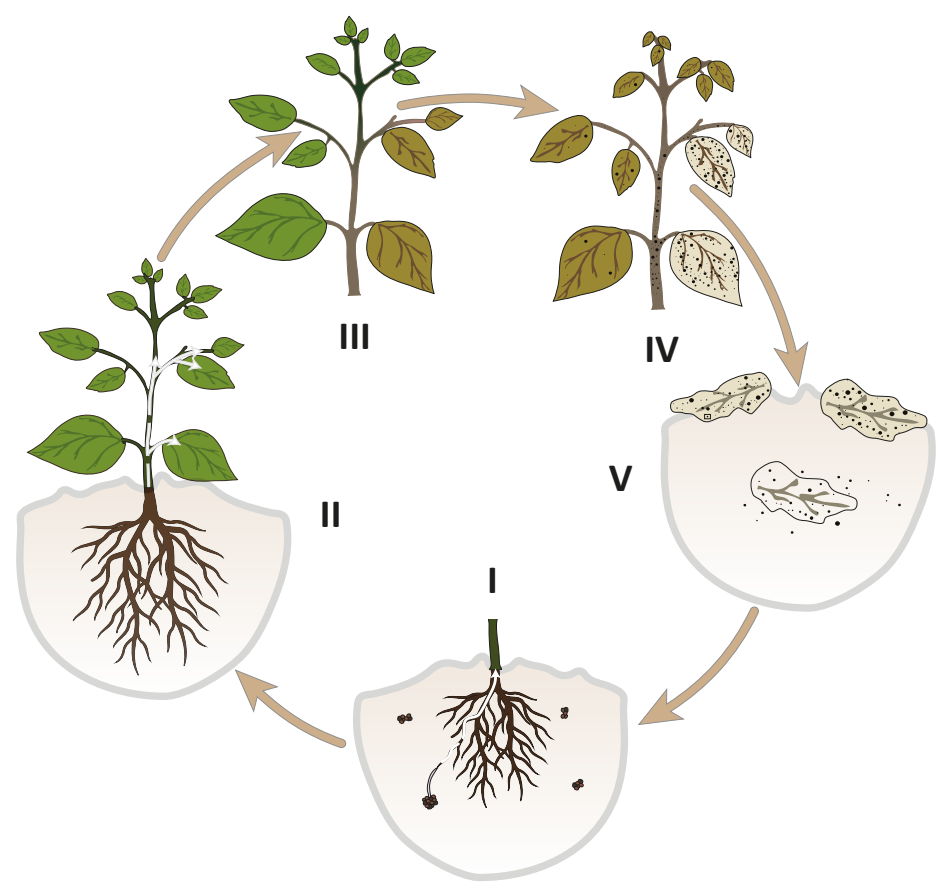

FIGURE 1 | Life cycle of the soil-borne broad host-range fungal plant pathogen Verticillium dahliae. The fungus survives in the soil through multicellular melanized resting structures named microsclerotia. Germination of these microsclerotia is stimulated by plant root exudates, after which the emerging hyphae grow saprophytically through the soil and rhizosphere to penetrate plant roots (I). Next, the fungus crosses the root cortex and enters the xylem vessels where sporulation occurs (II). The conidiospores are transported with the sap stream in the vasculature to distal plant tissues. Once conidiospores get trapped germination occurs, after which the fungus penetrates into new xylem vessels where sporulation re-occurs. This systemic colonization is accompanied by typical Verticillium wilt symptoms, including chlorosis, necrosis, and wilting (III). Once the tissue starts to senesce, V. dahliae emerges from the vasculature to colonize decaying host tissues where new microsclerotia are produced (IV). These microsclerotia are released into the soil upon tissue decomposition (V). 
Besides genetic resistance, biological control strategies to suppress $V$. dahliae and other pathogenic Verticillium spp. using antagonistic microbes have been explored extensively ${ }^{67}$. The identification of putative biological control agents to suppress $V$. dahliae is often initiated by the isolation of microbes from the endophytic compartment of corresponding host plants 67,68 , which are subsequently screened for their ability to inhibit the fungus in vitro or in planta. Such approaches have led to the identification of numerous microbial isolates displaying activities that could potentially affect $V$. dahliae during different life stages. For instance, various microbial antagonists have been reported to inhibit germination of $V$. dahliae microsclerotia or to outcompete Verticillium spp. for colonization of the host endosphere ${ }^{69,70}$. However, the majority of these biological control experiments have only been performed in vitro or under controlled greenhouse conditions, and transfer of biocontrol agents to the field has generally turned out less successful. It has become clear that in vitro antagonism typically correlates poorly with in planta activity $^{68}$. Consequently, putative biocontrol agents that display strong antagonism or mycoparasitism in vitro do not necessarily represent the best candidates in the field. Additionally, biocontrol experiments performed under controlled greenhouse conditions often depend on artificial Verticillium inoculation techniques and typically involve the application of a single biocontrol agent in sterilized soil ${ }^{67}$. Consequently, these approaches do not accurately reflect the conditions of Verticillium-infested soils in nature. As a result, the subsequent introduction of promising biocontrol agents in naturally infested soils often does not consistently suppress disease, as microbial antagonists have to establish themselves in a complex microbial community, which renders them less effective. Moreover, it can be anticipated that $V$. dahliae, as a soil-borne pathogen, evolved mechanisms to counteract microbial antagonists and competitors to survive in microbe-rich environments such as the soil and the root microbiome. Arguably, such mechanisms may potentially be overlooked under controlled in vitro or greenhouse conditions, while they may play a dominant role in nature.

\section{Thesis outline}

During my doctoral research I studied how $V$. dahliae exploits effector proteins to promote niche colonization through manipulation of the different microbiota it encounters during its life cycle.

Chapter 2 provides a literature review in which we provide fuel for the hypothesis that plant pathogens may secrete effector proteins to manipulate the composition of host microbiomes to their advantage in order to promote host colonization. Moreover, we argue that also other plant-associated microbes, such as endophytes and mutualists, may utilize effector proteins to facilitate host colonization. Additionally, we speculate 
that microbiota-manipulating effectors also promote niche colonization by free-living saprophytes or plant-associated microbes in the absence of a host. Finally, we discuss a number of strategies that may aid the identification of host microbiome-manipulating pathogen effector proteins.

In Chapter $\mathbf{3}$ we provide proof for the hypothesis that plant pathogens exploit effector proteins for the manipulation of host microbiomes. We show that the previously identified $V$. dahliae effector protein $V d A v e 1$ exerts antibacterial activity and facilitates host colonization through the manipulation of host microbiomes by suppressing bacterial antagonists of V. dahliae. Moreover, we show that VdAve1, and a newly identified antimicrobial effector protein named VdAMP2, are exploited for microbiome manipulation in the soil.

Antimicrobial activity assays performed in Chapter 3 revealed that VdAve1 affects various bacterial species, including the Gram-positive bacterium Bacillus subtilis. Using a combination of microscopic, transcriptomic and forward genetic analyses performed on B. subtilis, we describe in Chapter 4 that VdAve1 is a cell envelopeactive antimicrobial protein. Moreover, we provide evidence that points towards a lysozyme-like activity of the effector protein and we reveal an important role for teichoic acids in protection against VdAve1.

Many lysozymes affect bacteria through two distinct mechanisms. They hydrolyze the peptidoglycan in bacterial cell walls, and they simultaneously act as cationic antimicrobial proteins that perturb cell membranes. Chapter 5 addresses the hypothesis that $\mathrm{VdAve} 1$ exerts a lysozyme-like activity by assessing its potential to hydrolyze peptidoglycan as well as its ability to act as a non-enzymatic membranedisrupting cationic antimicrobial protein.

Functional characterization of $\mathrm{VdAve} 1$ and $\mathrm{VdAMP} 2$ revealed that $V$. dahliae dedicates part of its effector repertoire to microbiome manipulation and that microbiome-manipulating effectors can act during various stages of its life cycle. In Chapter 6 we describe the identification and characterization of a third antimicrobial $V$. dahliae effector, VdAMP3, and investigate its role during advanced $V$. dahliae infection stages.

Finally, Chapter $\mathbf{7}$ discusses the most important findings of this thesis. Moreover, it provides an outlook for the anticipated roles of microbiome-manipulating effectors in a broader context. 



\section{Chapter 2}

\section{Plant pathogen effector proteins as manipulators of host microbiomes?}

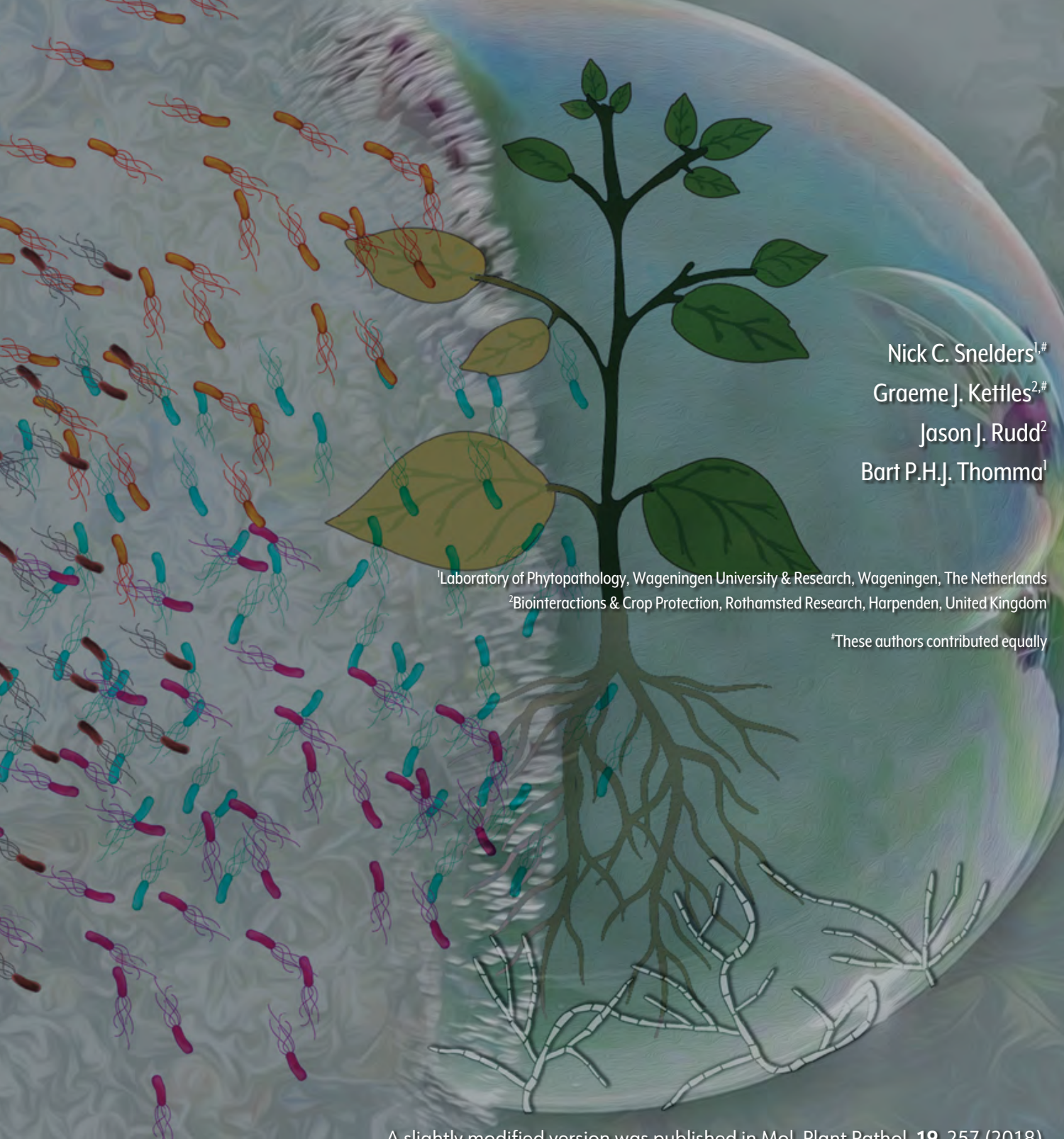

A slightly modified version was published in Mol. Plant Pathol. 19, 257 (2018). doi: $10.1111 / \mathrm{mpp} .12628$ 


\section{Plant pathogen effector proteins as manipulators of host microbiomes?}

To understand the mechanisms underlying disease development in plants, molecular plant pathology research has mostly focused on the characterization of direct interactions between plant pathogens and their hosts. Collectively, this research has demonstrated that plants sense microbial invaders using various types of receptors (recently coined as 'invasion pattern receptors', IPRs) that sense microbial invasion and activate defense responses upon recognition of various molecular patterns that betray microbial invasion (recently coined as 'invasion patterns', IPs) ${ }^{5}$. While these IPRs comprise cell surface-localized as well as intracellular receptors, IPs comprise microbeassociated molecular patterns (MAMPs) and other microbially secreted components, as well as host-derived damage-associated molecular patterns (DAMPs) ${ }^{5}$.

In order to successfully colonize their hosts and subvert immune responses, plant pathogens secrete molecules, so-called effectors, during attempted host ingress 5,25 . According to the initial, narrowest, definitions, effectors are small, cysteine-rich proteins that function through the manipulation of plant immune responses. However, ongoing research has revealed that effectors may have other functions as well, such as roles in pathogen self-defense or liberation of nutrients from host tissues ${ }^{25,71}$. Moreover, it is generally appreciated that other types of microbially secreted molecules, such as secondary metabolites and small RNAs ${ }^{72}$, may exert prototypical effector functions. Furthermore, it is accepted that effectors are not exclusively secreted by pathogens, as homologous molecules are employed by other types of symbiotic organisms, such as endophytes and mutualists, and even by saprophytes ${ }^{25}$. Consequently, more recently, it has been proposed that rather than being small, cysteine-rich proteins that function through the manipulation of plant immune responses, effectors should be defined as microbially secreted molecules that contribute to niche colonization ${ }^{25}$.

Similar to other higher organisms, plants associate with a plethora of microbes that collectively form its microbiota. The phyllosphere comprises all aerial parts of the plant and is commonly colonized by diverse microbial communities ${ }^{7}$. However, the most extensive microbial host colonization occurs below ground. The soil is a hotspot of microbial life, as microbial communities generally display great diversity and reach high densities. In particular, the narrow zone in close proximity to the roots, also known as the rhizosphere, is extremely microbe-rich as it attracts microbes from the surrounding soil and allows them to thrive on plant-derived root exudates ${ }^{13}$. Over recent years, the plant microbiota has gained increasing attention. Metagenomic studies have greatly enriched our knowledge of the composition of plant microbiomes, i.e. the microbes and their genomes in that environment ${ }^{6}$, and have led to its recognition as a key factor for plant health ${ }^{10}$. The role of the rhizosphere microbiome in disease suppression has been particularly well described. It is currently generally appreciated that plants exploit root exudates to increase microbial activity on pathogen attack, 
and specifically attract beneficial microbes from the very diverse microbial community residing in the bulk soil ${ }^{10}$. Consequently, plants select microbial communities around their roots that function as an additional layer of defense. One of the best-studied examples is the reduced incidence and severity of take-all disease caused by the fungus Gaeumannomyces graminis var. tritici which typically follows a severe disease outbreak in a monoculture of wheat or barley. This phenomenon is known as the socalled 'take-all decline' and is associated with the elevated presence of antagonistic Pseudomonas spp. that suppress the soil-borne fungal pathogen.

Like all microbes, plant pathogens are under strong selective pressure exerted by coinhabiting microorganisms. These microbiota members influence each other, both positively and negatively, through secreted molecules. A significant part of these molecules function through their antimicrobial activity and involve hydrolytic enzymes, antibiotics, toxins and volatiles ${ }^{22}$. In addition, microbes strongly compete with each other for nutrients and essential elements. Importantly, these processes often involve secreted molecules. Siderophores and haemophores are well-studied molecules secreted by plants and soil microbes to scavenge metal ions and facilitate their uptake 22 . Obviously, the above-mentioned antibiosis and competition for nutrients also impact microbial plant pathogens, and represent two important factors in disease suppression. However, other mechanisms responsible for disease suppression have also been reported. For example, beneficial rhizobacteria indirectly affect pathogens through the induction of systemic resistance in plants. Interestingly, rhizobacteria do so through various mechanisms, including the secretion of particular volatiles, antibiotics and siderophores that prime the plant's immune system for pathogen attack ${ }^{22}$.

To date, the study of the plant microbiome and biocontrol has exclusively investigated the influence of microbial communities on plant pathogens and host defense activation. However, the manipulation of these communities by plant pathogens in return, during host colonization to promote this process, as well as during free-living life stages outside the host, remains unexplored. Arguably, effector proteins may act as exquisite tools for the interaction with other microbes. This hypothesis may be supported by observations that, across numerous pathosystems, and despite significant effort, the functions of many effectors in terms of host plant manipulation remain unknown. Although this may derive from overlapping effector functionalities with plant targets, it may also be that some secreted protein effectors might instead be targeting the local microbial community. In addition to during host colonization, microbiota-manipulating effectors may also be important for saprophytic survival during free-living life stages outside the host. Arguably, non-pathogenic saprophytes may employ similar molecules to sustain themselves in the presence of other microbes, whereas endophytes and mutualists can be anticipated to secrete similar effectors to outcompete other microbes in the process of host colonization. With this in mind, effector proteins in general could be broadly classified into three groups: 
(1) plant-targeting effectors; (2) multifunctional effectors targeting plants and microbes; and (3) microbe-targeting effectors.

- Group (1) effectors have a role solely in the manipulation of the host organism. This includes pathogen proteins which suppress pathogen-associated molecular pattern-triggered immunity (PTI) and may be recognized in a gene-for-gene manner to induce either host resistance or susceptibility. It also includes effectors demonstrated to have multiple roles in host manipulation, such as the SnTox1 effector from Parastagonospora nodorum ${ }^{73}$.

- Group (2) effectors have roles in the manipulation of both the host and the local microbial community. Such a group is probably dominated by proteins with broad-spectrum activity targeting highly conserved physiological processes functional in both plants and microbes. For instance, effector proteins involved in self-defense towards antimicrobial components, such as hydrolytic enzymes secreted by plant hosts, can also be expected to offer protection against similar components secreted by competing microbes. In addition, plant pathogens can also be anticipated to secrete effector proteins with simultaneous phytotoxic and antimicrobial activity that affect both host and other microbes, such as the recently described Zt6 effector from the wheat pathogen Zymoseptoria tritici ${ }^{42}$.

- Group (3) effectors are highly specialized to target or disrupt processes specific to microbes, and thus may have distinct biochemical properties from those designed to target analogous mechanisms in plants. This group may also include those that act in an indirect fashion, for example by establishing local nutrient deprivation, or by affecting communication between plants and beneficial microbes. Finally, pathogens may secrete effectors to recruit cooperative microbes that offer protection against microbial competitors, or that aid in host colonization. This group of effectors may also play important roles for endophytic and saprophytic species.

Given their potential diversity, the task of identifying effector proteins involved in microbiota manipulation may appear to be daunting. However, expression profiling of genes coding for secreted proteins and direct identification by proteomics approaches of candidates during host colonization have proven to be successful for the identification of host-manipulating (group 1) effectors. Arguably, microbiotamanipulating effectors (groups 2 and 3 ) require different transcriptional triggers compared with the canonical effectors characterized to date, and probably display different transcriptional patterns compared with effectors dedicated purely to host manipulation. For example, elevated expression following complete colonization of the host may be unusual for host-manipulating (group 1) effectors, but may be commonplace for effectors intended to limit nutrient scavenging by competing microbes. Furthermore, distinct transcriptional signatures probably exist within each 
pathogen which may be highly dependent on pathogen lifestyle and exposure to microbial antagonists within a particular niche. Thus, transcriptomic approaches can be exploited to monitor the induction of effector genes under in vitro conditions that mimic microbial encounter. However, great care must be taken in the interpretation of these experiments.

It is possible that effector proteins that are relevant for survival in microbial communities are shared between closely related species that operate in the same niche. Hence, comparative genomics between saprophytic and pathogenic relatives can be used to identify core effector gene catalogs. For the effector categories introduced above, host-manipulating group 1 effectors probably belong to a single or small group of pathogen(s) and play highly specialized roles in the manipulation of perhaps a single (or small number of) host(s). Host- and microbe-targeting group 2 effectors probably exhibit a broader distribution not only amongst plant pathogens, but also amongst non-pathogenic species, because of their ability to influence microbe-microbe interactions. Finally, microbe-targeting group 3 effectors probably display the broadest distribution of all, encompassing plant pathogens, endophytes and saprophytes. To this end, transcriptomic analyses and comparative genomics approaches complement each other and can be used in parallel to identify relevant effector candidates. Subsequently, functional screens aimed to determine their direct effect on other microbes should reveal whether or not the effector candidates have potential microbiota-manipulating abilities. An initial (and potentially overlooked) medium- to high-throughput screen might be to first test whether candidate proteins can be expressed in either prokaryotic or eukaryotic recombinant expression systems. Our recent discovery of the multifunctional Zt6 effector from Z. tritici initially came from our inability to express full-length recombinant protein in either Escherichia coli or Pichia pastoris expression systems, potentially due to toxicity ${ }^{42}$. This contrasts with most other tested $Z$. tritici candidate effectors, which express relatively well in either system, albeit often to different levels. The availability of specialist $E$. coli expression strains (e.g. SHuffle, New England Biolabs, Ipswich, MA, USA), designed to express cysteine-rich eukaryotic proteins with minimal inclusion body formation, makes this toxicity screen viable. Although this type of screen based on negative results is probably not optimal, it could be used as a baseline for then testing the relative ability to generate subtle mutant versions of these proteins for more direct testing, or to test for toxicity responses via other transient expression systems, perhaps even using agroinfiltration as a route to determine whether the toxicity is broad or selective. It may even be possible to recover protein from such a system for direct testing on microbes. In addition, screens can be aimed at the identification of effector candidates involved in the recruitment of cooperative microbes by determining their ability to promote the growth of other microbial species. Finally, irrespective of observations during initial screens, gene functional analysis will be required to validate the relevance of the effectors in the biological context and to confirm their role in microbial interactions. 
Unveiling the roles of plant pathogen effector proteins in the manipulation of microbiota will add to our fundamental understanding of the mechanisms contributing to disease establishment, and could potentially lead to improved disease control methods. Current crop disease control is heavily reliant on the application of synthetic fungicides and bacteriocides. However, pathogen resistance to chemical control has, in some cases, become widespread. In addition, soil-borne pathogens are especially difficult to control because of their persistent resting structures. Therefore, the biocontrol of plant pathogens using antagonistic microbes is an alternative option. Nevertheless, the biocontrol of pathogens is not always consistent and could be improved to become a more reliable disease control method. To this end, the characterization of microbiota-manipulating effectors can contribute to more targeted biocontrol strategies, as it allows for the selection of antagonistic microbes that are insensitive to, or can interfere with, the activities of pathogen effectors. In addition, similar to previously identified effector proteins, plants may have evolved IPRs to recognize microbiota-manipulating effectors and their activities. Microbiota-manipulating effectors thus represent an interesting pool of unexplored avirulence factors for which recognition in particular plant genotypes may exist and may help to identify or engineer novel immune receptors that may contribute to improved pathogen resistance in crops. Finally, given that many microbes secrete antimicrobial molecules, but are themselves immune, what are the mechanisms of self-protection? An understanding of these fundamental aspects of microbe-microbe interactions on plants may provide a future source of targets for intervention and disease control.

\section{Acknowledgements}

Work in the laboratory of B.P.H.J.T. is supported by the Research Council Earth and Life Sciences (ALW) of the Nederlandse Organisatie voor Wetenschappelijk Onderzoek (NWO). G.J.K. and J.J.R. were supported by the strategic grant 'Designing Future Wheat' (grant no. BB/P016855/1) from the Biotechnology and Biological Sciences Research Council of the UK (BBSRC) and by the Rothamsted Institute Fellowship Program. The authors are grateful for the support of the COST action SUSTAIN (FA1208): Pathogen-informed strategies for sustainable broad-spectrum crop resistance. The authors declare no conflicts of interest. 


\section{Chapter 3}

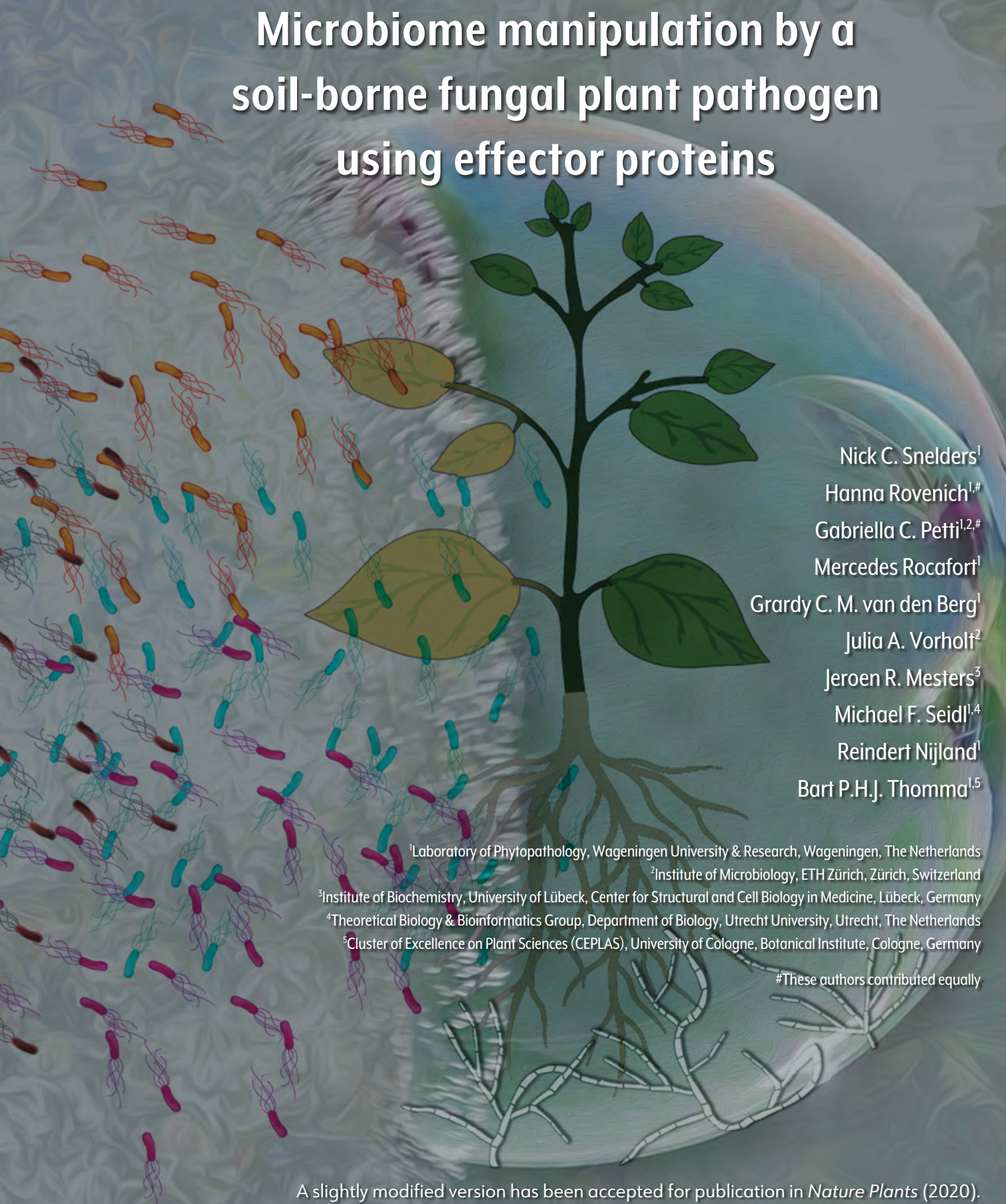




\begin{abstract}
During colonization of their hosts, pathogens secrete effector proteins to promote disease development through various mechanisms. Increasing evidence shows that the host microbiome plays a crucial role in health, and that hosts actively shape their microbiomes to suppress disease. We hypothesized that pathogens evolved to manipulate host microbiomes to their advantage in turn. Here, we show that the previously identified virulence effector $\mathrm{VdAve} 1$, secreted by the fungal plant pathogen Verticillium dahliae, displays antimicrobial activity and facilitates colonization of tomato and cotton through the manipulation of their microbiomes by suppressing antagonistic bacteria. Moreover, we show that $\mathrm{VdAve1}$, and also the newly identified antimicrobial effector VdAMP2, are exploited for microbiome manipulation in the soil environment, where the fungus resides in absence of a host. In conclusion, we demonstrate that a fungal plant pathogen utilizes effector proteins to modulate microbiome compositions inside and outside the host, and propose that pathogen effector catalogs represent an untapped resource for novel antibiotics.
\end{abstract}




\section{Introduction}

To establish disease, pathogenic microbes secrete a wide diversity of effector proteins that facilitate host colonization through a multitude of mechanisms ${ }^{25}$. Typically, pathogen effectors are defined as small cysteine-rich proteins that are secreted upon colonization to manipulate host physiology or to deregulate host immune responses ${ }^{74}$. Consequently, effector proteins are predominantly studied in binary host-microbe interactions, while largely ignoring the biotic context in which these interactions take place. Higher organisms, including plants, associate with a plethora of microbes that form their microbiota, which represents a key determinant for their health ${ }^{14,41,75-77}$. The most extensive microbial colonization of plants occurs at roots, where plants define rhizosphere microbiota compositions through secretion of exudates ${ }^{16,17}$ and specifically attract beneficial microbes to suppress pathogen invasion ${ }^{10,15,20}$. Thus, we hypothesized that plant pathogens evolved mechanisms to counteract this recruitment and modulate host microbiomes, comprising the microbes and their genomes in their environment ${ }^{6}$, for successful infection, possibly through effector proteins ${ }^{25,78}$.

Verticillium dahliae is a soil-borne fungus that causes vascular wilt disease on hundreds of plant species, including numerous $\operatorname{crops}^{47,48}$. V. dahliae survives in the soil through persistent resting structures called microsclerotia that germinate in response to nutrient-rich exudates released by nearby plant roots ${ }^{55}$. Subsequently, emerging hyphae grow through the soil and rhizosphere towards the roots where the fungus penetrates its hosts. Following root penetration, $V$. dahliae invades the xylem where it produces conidiospores that are spread throughout the vasculature by the sap stream. This systemic colonization causes chlorosis and necrosis of plant tissues, which is followed by plant senescence. $V$. dahliae then enters a saprophytic phase, emerges from the vasculature and colonizes the dead plant material where it produces new microsclerotia that are eventually released into the soil upon tissue decomposition.

Using comparative population genomics, we previously identified the $V$. dahliaesecreted small cysteine-rich effector protein Ave 1 that is recognized as an avirulence determinant by tomato plants that carry the corresponding Ve1 immune receptor ${ }^{61}$. However, on host plants lacking Ve1, VdAve1 acts as a virulence effector that promotes fungal colonization and disease development ${ }^{61}$. Interestingly, VdAve 1 is homologous to plant natriuretic peptides (PNPs) that have been identified in numerous plant species, suggesting that $V d A v e 1$ was acquired from plants through horizontal gene transfer ${ }^{61}$. Whereas several of the plant PNPs were shown to act in plant homeostasis and (a)biotic stress responses ${ }^{79,80}$, the mode of action of $\mathrm{VdAve} 1$ to contribute to fungal virulence has remained unknown. 


\section{Results}

Unlike most pathogen effector genes characterized to date, the $V$. dahliae effector gene Ave 1 is not only highly expressed during host colonization ${ }^{61,81}$, but also during growth in vitro and under conditions mimicking soil colonization, suggesting a ubiquitous role throughout the fungal life cycle including life stages outside the host, and thus a role that does not primarily involve targeting host plant physiology (Supplementary Fig. 1). Our attempts to purify VdAve1 upon heterologous expression in Escherichia coli, to facilitate functional characterization, repeatedly failed due to the formation of inclusion bodies (Supplementary Fig. 2a). The inability to obtain soluble protein using heterologous microbial expression systems can be attributed to a multitude of reasons, but is a well-known phenomenon when expressing antimicrobial proteins ${ }^{82}$. Consequently, based on the ubiquitous expression of $V d$ Ave 1 by $V$. dahliae, and our inability to purify soluble VdAve1 following expression in E. coli, we hypothesized that VdAve1 may possess antimicrobial activity.

To obtain functional VdAve1, inclusion bodies were isolated from E. coli cells and denatured using guanidine hydrochloride. Next, VdAve1 was refolded by stepwise dialysis and functionality was confirmed through testing recognition by its immune receptor Ve1 (Supplementary Fig. 2b). To assess the potential antimicrobial activity of VdAve1, we developed an in vitro system in which we incubated a panel of plantassociated bacteria in tomato xylem fluid, to mimic a natural environment in which VdAve1 is secreted, namely tomato xylem vessels, and monitored their growth in presence and absence of the protein. Interestingly, VdAve1 selectively inhibited the growth of plant-associated bacteria (Fig. 1a). Whereas growth of all Gram-positive bacteria tested, namely Arthrobacter sp., Bacillus subtilis, Staphylococcus xylosus and Streptomyces sp., was strongly inhibited, Gram-negative bacteria displayed differential sensitivity to the protein. Intriguingly, this differential sensitivity is not immediately explained by phylogenetic relationships of the tested isolates as even within bacterial orders/families differences are observed. For instance, whereas growth of the burkholderiales species Acidovorax is inhibited by VdAve1, growth of a Ralstonia isolate, which belongs the same order, is not. Similarly, treatment of two closely related rhizobiales, Rhizobium sp. and Agrobacterium tumefaciens, revealed differential sensitivity as VdAve1 affected growth of Rhizobium sp., but not of $A$. tumefaciens. Finally, growth of Pseudomonas corrugata and Serratia sp. was only slightly altered and unaffected, respectively, while growth of both Sphingobacterium sp. and Sphingomonas mali was affected upon exposure to VdAve1. Interestingly, treatment of a panel of fungal species with $\mathrm{VdAve} 1$ revealed no antifungal activity of the effector, whereas treatment of plant protoplasts could not reveal phytotoxic activity, suggesting that VdAve1 exclusively acts on bacteria. (Supplementary Fig. $3 a, b)$. Our initial observations with divergent, randomly chosen, plant-associated bacteria prompted us to further characterize the antimicrobial activity of $\mathrm{VdAve} 1$. 
As a first step in the further characterization of the antimicrobial activity of VdAve1, we aimed to determine whether the effector protein is bacteriostatic or bactericidal by making use of electron microscopy to visualize the effect of protein treatment on bacteria. As a target species the Gram-positive $B$. subtilis was chosen, considering its high sensitivity to $\mathrm{VdAve} 1$ treatment. By testing a concentration series of the $\mathrm{VdAve} 1$ effector protein, the minimum inhibitory concentration (MIC) was determined at $8 \mu \mathrm{M}$ (Supplementary Fig. 3c). However, electron microscopy analysis revealed that subMIC concentrations of VdAve1 already induced blebbing and swelling of bacterial cells, followed by lysis and collapse, indicating that the antimicrobial activity of $\mathrm{VdAve} 1$ might be based on bactericidal activity (Fig. 1b).

To investigate whether the antimicrobial activity that is displayed by VdAve1 is more widely conserved among its homologs, we tested the only homolog that occurs in one of the sister species of the Verticillium genus, namely VnAve1, from the nonpathogenic species $V$. nubilum that displays $90 \%$ amino acid identity (Supplementary Fig. 3d). Interestingly, also this homolog displays antimicrobial activity, but it only inhibits a subset of the bacteria affected by VdAve1, and does not cause $B$. subtilis lysis (Fig. 1). Thus, the 13 amino acid polymorphisms between the two Ave1 homologs are responsible for differences in the activity spectrum. To investigate whether the antimicrobial activity also occurs among plant homologs, or is confined to microbial homologs and involves neofunctionalization after horizontal transfer, the more distant homolog AtPNP-A from Arabidopsis thaliana was tested as well. Intriguingly, AtPNP-A completely arrests $B$. subtilis growth (Supplementary Fig. 3d,e). Collectively, these findings demonstrate that various Ave1 homologs possess antimicrobial activity, yet with divergent activity spectra, and suggest that the antimicrobial activity of $\mathrm{VdAve} 1$ did not result from neofunctionalization following horizontal gene transfer.

Based on the strong but selective bactericidal activity of $\mathrm{VdAve} 1$ in vitro, we hypothesized that $V$. dahliae exploits its effector protein to affect host microbiome compositions through the suppression of other microbes. Therefore, to determine the biological relevance of the observed bactericidal activity, we performed bacterial community analysis based on $16 \mathrm{~S}$ ribosomal DNA profiling of tomato and cotton root microbiomes following infection with wild-type $V$. dahliae or a VdAve1 deletion mutant. Importantly, root microbiome compositions were determined during early $V$. dahliae infection stages, namely at ten days post inoculation when the fungus has just entered xylem vessels and initiated systemic spreading, to minimize indirect shifts in microbial compositions that result from severe disease symptomatology, rather than from direct shifts due to the presence of the effector protein. We did not observe major shifts in overall composition of bacterial phyla (Supplementary Fig. 4a) or total microbial diversity (a-diversity) (Supplementary Fig. 4b) upon $V$. dahliae colonization of tomato and cotton. However, principal coordinate analysis based on Bray-Curtis dissimilarities ( $\beta$-diversity) revealed a clear 
separation of root microbiomes (Fig. 2a) (PERMANOVA, $p<0.01$ for both tomato and cotton). Importantly, the extent of $V$. dahliae colonization does not seem to determine the separation, as clustering of $V$. dahliae genotypes occurs in cotton although VdAve1 deletion hardly affects fungal virulence on this host plant (Fig. 2a).

a
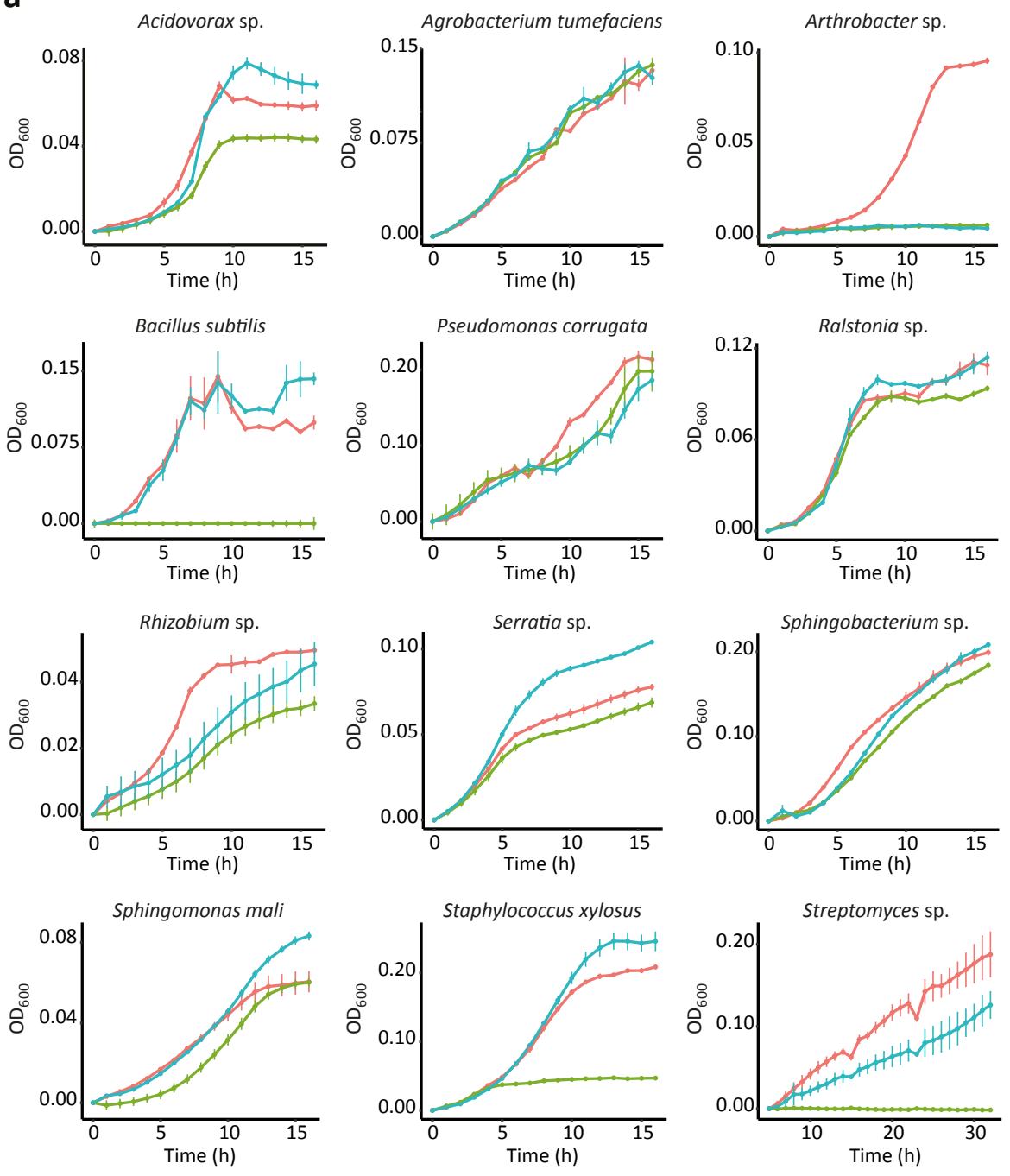

$+\mathrm{MQ}+8 \mu \mathrm{M}$ VdAve1 $+8 \mu \mathrm{M}$ VnAve1 
b

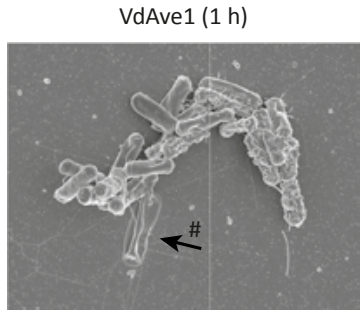

VdAve1 (4 h)

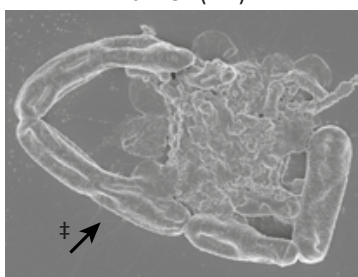

VdAve1 (2 h)

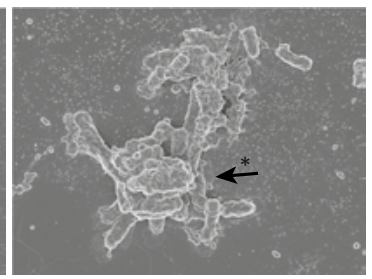

VdAve1 (8 h)

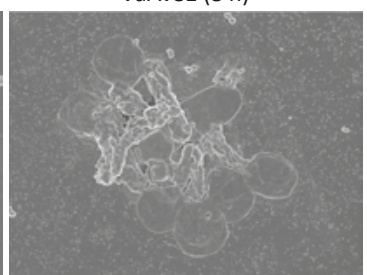

Water

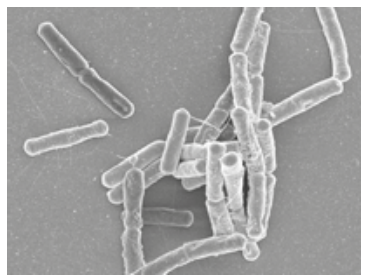

VnAve1 (8h)

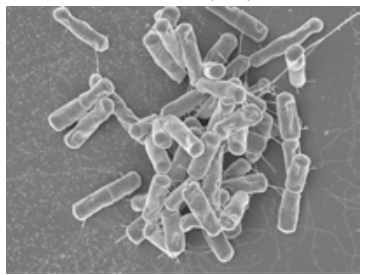

$2 \mu \mathrm{m}$

FIGURE 1 | Antibacterial activity of Verticillium dahliae effector VdAve1. (a) VdAve1 selectively inhibits in vitro growth of plant-associated bacterial isolates in tomato xylem fluid. The close homolog VnAve1 from $V$. nubilum only inhibits a subset of the bacteria affected by VdAve1 and is generally less effective. Ultrapure water $(\mathrm{MQ})$ was used as control. Graphs display the average $\mathrm{OD}_{600}$ of three biological replicates \pm SD. (b) Scanning electron microscopy of $B$. subtilis upon 1, 2, 4 and 8 hours of incubation in tomato xylem fluid showing blebbing $\left({ }^{*}\right)$, swelling ( $\left.¥\right)$ and lysis $(\#)$ with $6.5 \mu \mathrm{M}$ VdAve1 $(0.8 \times \mathrm{MIC})$, but not with water or VnAve1.

Thus, as anticipated based on the potent, yet selective, antimicrobial activity, VdAve 1 secretion by $V$. dahliae sophistically alters root microbiome compositions. However, strikingly, despite the relatively small sample size of our $16 \mathrm{~S}$ rDNA profiling, pairwise bacterial order comparisons upon colonization by wild-type $V$. dahliae and the VdAve1 deletion mutant revealed differential abundances of Sphingomonadales, Bdellovibrionales and Ktedonobacterales for tomato (Fig. 2b) (Supplementary Table 1). The finding that Sphingomonadales are repressed in the presence of VdAve1 suggests that this taxon is the most sensitive to VdAve1 activity. A similar comparison for cotton did not immediately reveal any differentially abundant orders, but agglomeration of amplicon sequence variants (ASVs) based on phylogenetic relatedness (patristic distance $<0.1$ ) revealed eight differentially abundant taxa, including a taxon of the Sphingomonadaceae family (Fig. 2b) (Supplementary Table 2). Interestingly, although this taxon only represents a small proportion of all Sphingomonadaceae in the cotton root microbiomes, it is exclusively and consistently found in the microbiomes of roots infected by the VdAve 1 deletion mutant, and completely absent upon infection with wild-type $V$. dahliae, again pointing towards the particular sensitivity of this taxon to VdAve1. Moreover, pairwise comparisons following the combination of tomato and cotton samples based on infection by the 
different $V$. dahliae genotypes, to identify differentially abundant bacterial orders that potentially remained unnoticed due to the limited sample size, again only revealed differential abundance of Sphingomonadales $(p<0.01$; Supplementary Fig. 4c) (Supplementary Table 3). Given the fact that secretion of VdAve1 by V. dahliae during colonization of both tomato and cotton leads to a reduction of Sphingomonadales in the corresponding root microbiomes, we anticipated a broad efficacy of $\mathrm{VdAve} 1$ on bacteria within this order. Thus, to identify Sphingomonadales genera that are most sensitive to VdAve1, we identified ASVs with increased average relative abundance in the microbiomes with the $V d A v e 1$ deletion mutant when compared with wild-type V. dahliae, revealing Sphingomonas, Novosphingobium, Sphingopyxis and Sphingobium that are commonly referred to as Sphingomonads (Fig. 2c) $)^{83,84}$.

To test whether $\mathrm{VdAve} 1$ is indeed able to directly manipulate microbial communities, and to confirm that root microbiome compositional changes are not due to indirect effects through host manipulation, we treated a synthetic community comprising plant-associated bacteria with purified $\operatorname{VdAve} 1$ and determined changes in the microbial communities using $16 \mathrm{~S}$ ribosomal DNA profiling. As anticipated, VdAve1 clearly impacted the composition of the microbial communities (Fig. 2d) (Supplementary Fig. 5). Interestingly, pairwise differential abundance analyses of bacterial orders again revealed a significant repression of Sphingomonadales in the presence of VdAve1 (adjusted p<0.05) (Fig. 2e) (Supplementary Table 4). Collectively, these findings confirm the ability of VdAve1 to directly manipulate microbiome compositions, and further substantiate the previously observed impact on Sphingomonadales in planta as a direct consequence of VdAve1 activity.

Transcriptional analysis revealed that VdAve 1 is not only highly expressed in planta, but also during growth conditions mimicking soil colonization, suggesting that the antimicrobial activity of $\mathrm{VdAve} 1$ also facilitates $V$. dahliae niche colonization outside the host. Indeed, $V$. dahliae colonization assays performed in soil, or in MS medium supplemented with soil, demonstrated that VdAve 1 contributes to $V$. dahliae fitness (i.e. biomass accumulation) in the presence of soil microbiota (Fig. 2f). Importantly, no such contribution was detected in the absence of soil microbes, indicating that niche colonization promoted by $\operatorname{VdAve} 1$ is based on its antimicrobial activity. Accordingly, as inferred from principal coordinate analysis based on Bray-Curtis dissimilarities of the microbiomes in the MS medium, secretion of VdAve1 indeed affected community structures, an effect that could be detected less clearly in soil, arguably due to much stronger dilution effects (Fig. 2g) (Supplementary Fig. 5).

To confirm the anticipated sensitivity, a panel of plant-associated Sphingomonads was incubated with VdAve1 in vitro 8,85 . In accordance with the previously observed effect on S. mali (Fig. 1a), treatment with VdAve1 was found to also inhibit growth 
of Sphingobium, Novosphingobium, Sphingopyxis and two other Sphingomonas species (Fig. 3a), indicating a broad sensitivity among the Sphingomonads. Given the selective efficacy of VdAve1 and the strong effect on Sphingomonads in the tomato and cotton microbiota, we hypothesized that these bacteria may act as antagonists and negatively affect $V$. dahliae growth in the absence of VdAve1. Indeed, co-cultivation of $V$. dahliae with Novosphingobium sp. A, as well as with $S$. macrogoltabida, resulted in reduced fungal biomass of the $V d A v e 1$ deletion mutant when compared with the $V$. dahliae wild-type that secretes VdAve1 under these conditions, revealing that Sphingomonads comprise antagonists of $V$. dahliae, and explaining the importance of their inhibition by VdAve1 (Fig. 3b). Importantly, cocultivation with VdAve1-insensitive Agrobacterium, Pseudomonas and Ralstonia isolates did not affect biomass accumulation of the VdAve 1 deletion mutant when compared with the wild-type, indicating that the detected differences in the presence of the Sphingomonads are indeed VdAve1 dependent (Supplementary Fig. 6a). Accordingly, pre-treatment of surface-sterilized tomato seeds with Ralstonia sp. did not impact Verticillium wilt disease development. In contrast, and in line with previously described observations of plant protective activities of Sphingomonad strains ${ }^{8}$, pre-treatment of surface-sterilized tomato seeds with S. macrogoltabida negatively affected Verticillium wilt disease development as confirmed through biomass quantification of wild-type $V$. dahliae in the presence and the absence of the bacterium (Fig. 3c,d) (Supplementary Fig. 7). Importantly, quantification of S. macrogoltabida in the presence of wild-type $V$. dahliae and the VdAve1 deletion mutant using $16 \mathrm{~S}$ rDNA profiling and real-time PCR revealed that VdAve1 secretion significantly impacts $S$. macrogoltabida proliferation to counter its protective effect (Fig. $3 \mathrm{e}-\mathrm{g}$ ). Notably, this observation is not an indirect effect of differential host colonization by wild-type $V$. dahliae and the $V d A v e 1$ deletion mutant, as selection of tomato plants with equal levels of $V$. dahliae biomass (Fig. $3 d$, data points highlighted in red), reveals similarly impaired $S$. macrogoltabida proliferation in the presence of VdAve1 (Fig. 3g). Thus, these data underpin the hypothesis that $V$. dahliae secretes the VdAve1 effector to target antagonistic bacteria, including Sphingomonadales, during host colonization, although it needs to be acknowledged that ideally our hypothesis would be tested in experiments based on V. dahliae inoculation of germfree tomato plants, a system we have not managed to establish thus far. 

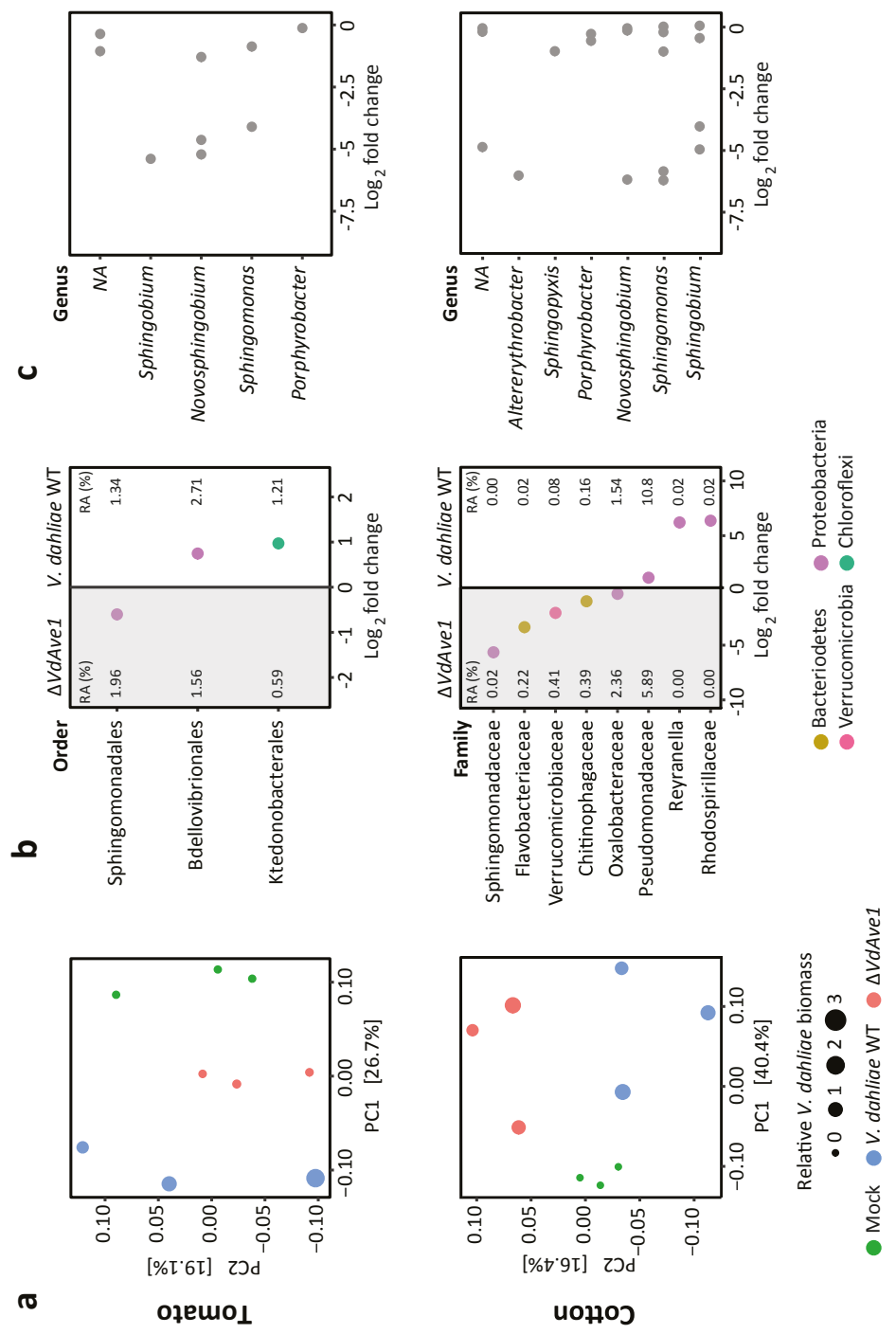


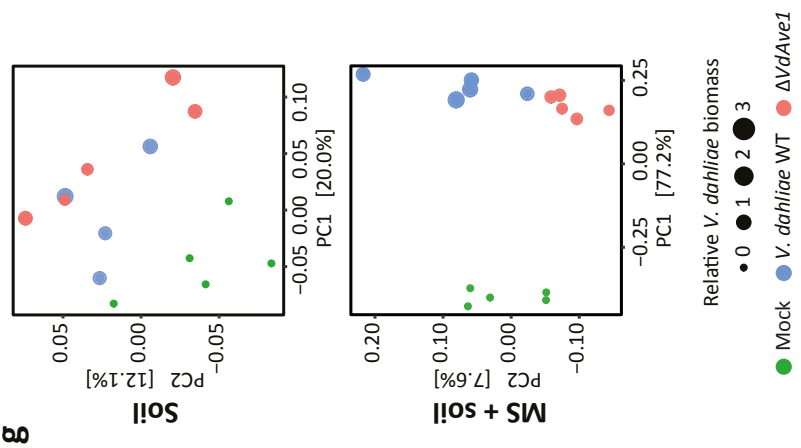

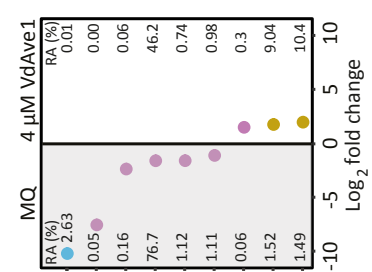
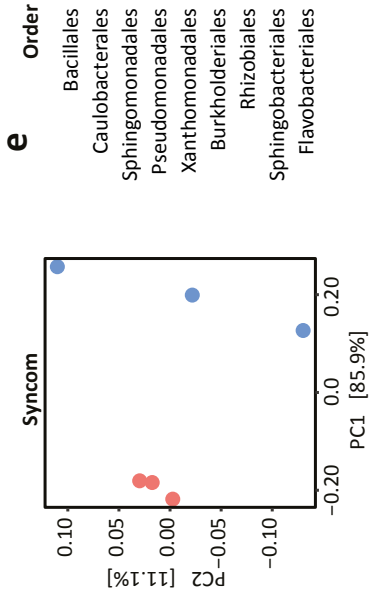

bo

I!OS
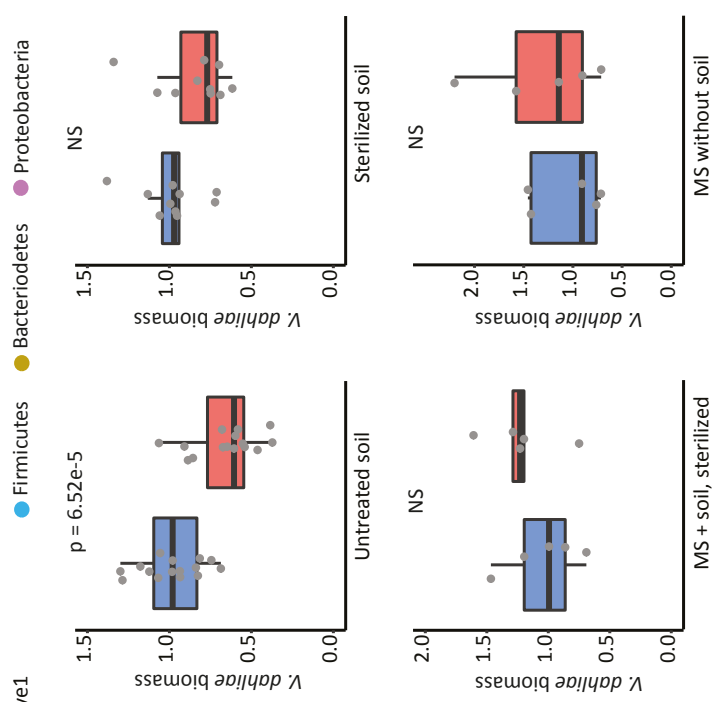

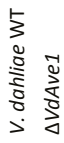

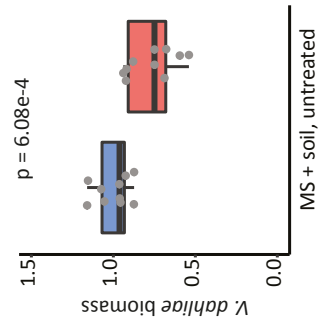

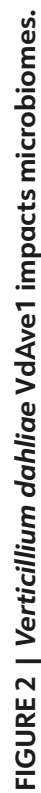


FIGURE 2 | Verticillium dahliae VdAve1 impacts microbiomes. (a) Principal coordinate analysis based on Bray-Curtis dissimilarities reveals separation of root microbiome compositions ten days after inoculation with wild-type $V$. dahliae and a VdAve1 deletion mutant (PERMANOVA, $p<0.01, N=3$ ). Roots with rhizosphere soil from three tomato or two cotton plants were pooled to form a single biological replicate. (b) Differential abundance analysis of bacterial orders (tomato) and upon agglomeration of amplicon sequence variants (patristic distance $<0.1$ ) (cotton) through pairwise comparison between root microbiomes colonized by wild-type $V$. dahliae and a VdAve1 deletion mutant (Wald test, $\mathrm{p}<0.01$ ). The average relative abundance (RA) of the differentially abundant taxa is indicated as a percentage of the total bacterial community in the corresponding root microbiome. (c) Sphingomonads (Sphingomonas, Novosphingobium, Sphingopyxis, and Sphingobium) are repressed by VdAve1. Dots represent single amplicon sequence variants with increased abundance (average of 3 samples) in root microbiomes upon colonization by the VdAve1 deletion mutant when compared with wild-type $V$. dahliae. (d) Principal coordinate analysis based on Bray-Curtis dissimilarities reveals separation of synthetic community compositions based on treatment with $4 \mu \mathrm{M}$ purified VdAve1 (N=3). (e) Differential abundance analysis of bacterial orders upon agglomeration of amplicon sequence variants through pairwise comparison of the synthetic communities treated with demineralized water or $4 \mu \mathrm{M} \mathrm{VdAve1}$ (Wald test, adjusted $\mathrm{p}<0.05$ ). (f) VdAve1 contributes to soil colonization. $V$. dahliae biomass in soil samples $(\mathrm{N}=15)$ or liquid MS medium supplemented with soil $(N=10)$ was determined by real-time PCR seven or three days after inoculation with wild-type $V$. dahliae (WT) and the VdAve 1 deletion mutant, respectively. P-values indicate statistically significant differences according to unpaired two-sided student's t-test. VdAve1 does not contribute to $\mathrm{V}$. dahliae colonization in sterile soil ( $N=10)$ and sterile MS medium with or without soil $(\mathrm{N}=5)$. (g) Principal coordinate analysis based on Bray-Curtis dissimilarities reveals clear separation of microbial community compositions in MS medium supplemented with soil (PERMANOVA, $\mathrm{p}<0.01$ ), but not in soil. $\mathrm{N}=5$ for all experimental conditions except for soil with $V$. dahliae WT for which $N=4$.

Our observation that $V$. dahliae secretes VdAve1 to suppress microbial competitors in the microbiota of its hosts, prompted us to speculate about additional $V$. dahliae effector proteins involved in microbiome manipulation. Therefore, to query for the occurrence of additional effectors that aid in microbial competition, the predicted secretome of $V$. dahliae strain JR2 ${ }^{86}$ was probed for structural homologs of known antimicrobial proteins (AMPs), revealing 10 candidates (Supplementary Table 5). The majority of the identified effectors share typical characteristics with canonical host-targeting effector proteins, such as being small and rich in cysteines. However, based on previously performed RNA sequencing experiments, no expression of any of these candidates could be monitored during colonization of Arabidopsis thaliana, Nicotiana benthamiana or cotton plants (Supplementary Fig. 8)61,81,87,88. Additionally, in vitro cultivation of $V$. dahliae in the presence of $E$. coli, B. subtilis or $T$. viride, or of peptidoglycan to mimic bacterial encounter, did not lead to induction of any of the effector candidate genes (Supplementary Fig. 8). Consequently, we hypothesized that these genes require other environmental triggers to be induced. Indeed, growth in soil extract consistently induced expression of candidate VdAMP2 (Fig. 4a) that shares structural homology (confidence $>90 \%$ ) with amphipathic $\beta$-hairpins of aerolysin-type $\beta$-pore forming toxins ( $\beta$-PFTs) (Supplementary Fig. 9a) ${ }^{89}$. 
a
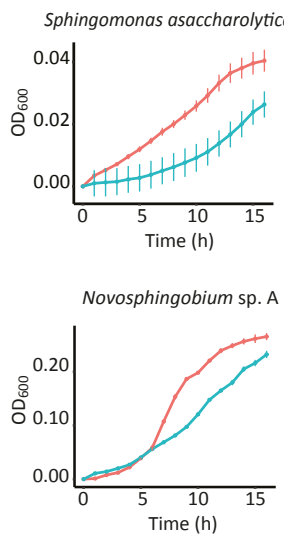

C

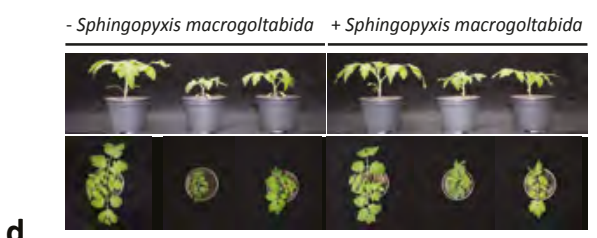

d
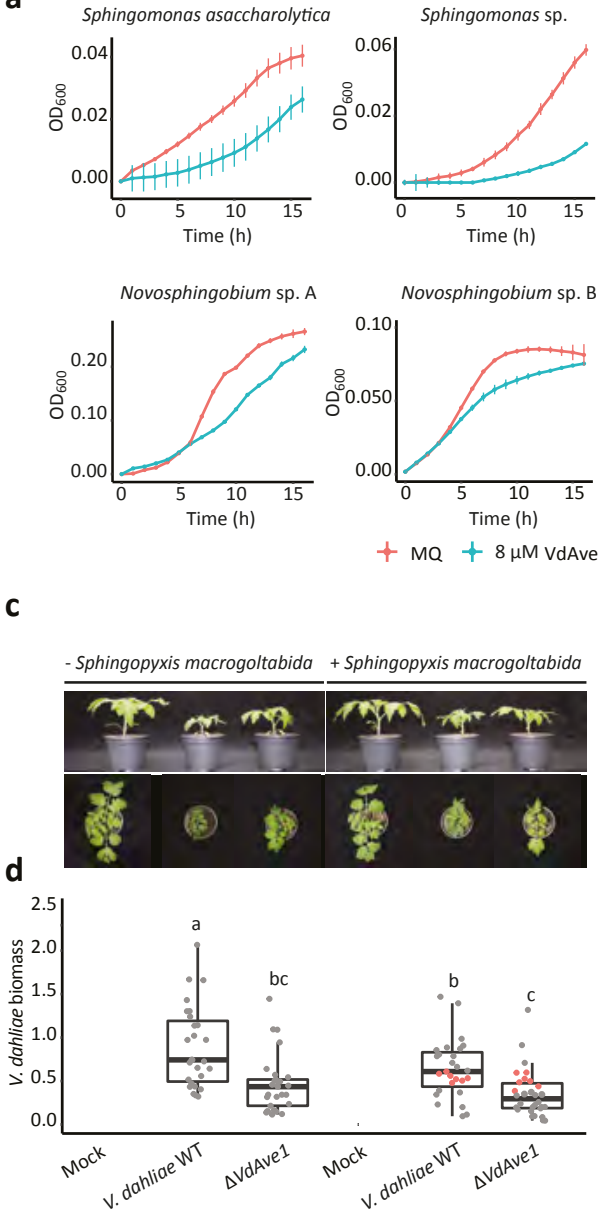

Sphingobium sp.

$+\mathrm{MQ}+8 \mu \mathrm{M}$ VdAve1

e

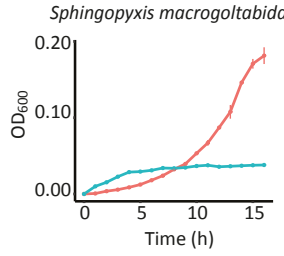

e

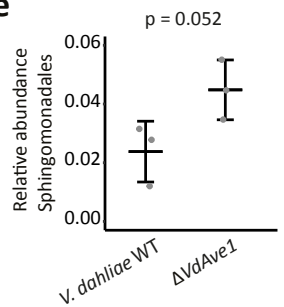

f

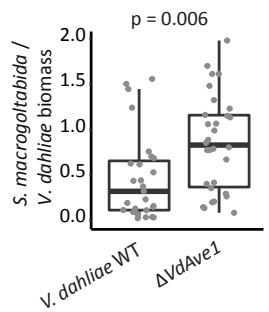

b
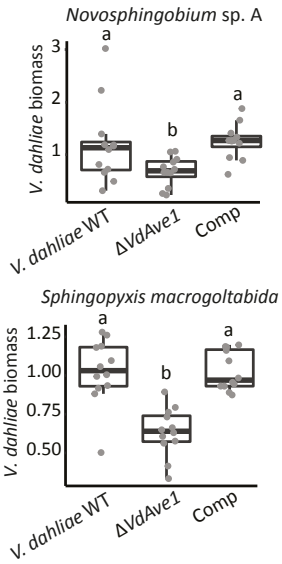

g

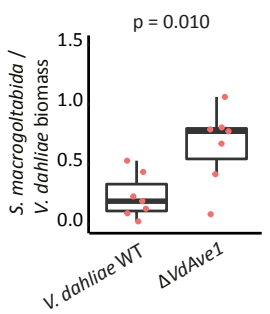

FIGURE 3 | Verticillium dahliae VdAve1 affects antagonistic Sphingomonads. (a) Sphingomonads are inhibited by VdAve1 in tomato xylem fluid. Graphs display the average $\mathrm{OD}_{600}$ of three biological replicates \pm SD. (b) VdAve1 supports $V$. dahliae growth in the presence of Sphingomonads. Biomass of wild-type $V$. dahliae (WT) and the VdAve1 deletion ( $\triangle V d A v e 1)$ and complementation (Comp) mutants was quantified following 48 hours of co-cultivation with Sphingomonads in $0.5 \times$ MS medium $(\mathrm{N}=12)$. Letters represent significant differences (one-way ANOVA and Tukey's post-hoc test; $p<0.05$ for Novosphingobium sp.; $\mathrm{p}<0.0001$ for S. macrogoltabida). (c) Tomato seed treatment with S. macrogoltabida reduces Verticillium wilt symptoms (stunting; 14 days post inoculation). (d) $V$. dahliae biomass in tomato stems determined with real-time PCR. Letters represent significant biomass differences (one-way ANOVA and Tukey's posthoc test; $p<0.05 ; \mathrm{N} \geq 27$ ). Each dot, grey or red, indicates the relative $V$. dahliae biomass in a single tomato plant. (e) Relative abundance of Sphingomonadales according to $16 \mathrm{~S}$ ribosomal DNA profiling of tomato plants pre-treated with S. macrogoltabida and infected with wild-type V. dahliae or the VdAve1 deletion mutant (unpaired two-sided student's t-test; $\mathrm{N}=3$ ). The plot displays the average relative abundance $\pm \mathrm{SD}$. (f) Relative Sphingopyxis biomass in all pre-treated tomato plants infected with wild-type $V$. dahliae or the VdAve 1 deletion mutant, indicated by the grey and red dots in (d) combined, as quantified by real-time PCR (unpaired two-sided student's t-test; N 227 ). (g) Relative Sphingopyxis biomass in pre-treated tomato plants colonized by similar amounts of wild-type $\mathrm{V}$. dahliae or the VdAve 1 deletion mutant, indicated by the red dots (d), as quantified by real-time PCR (unpaired two-sided student's t-test; $N=7$ ). 


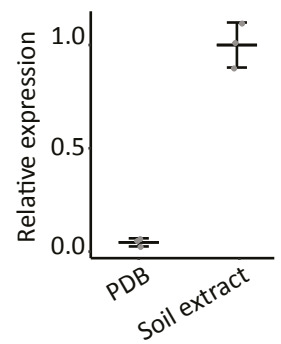

C

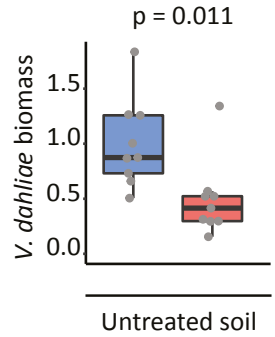

b

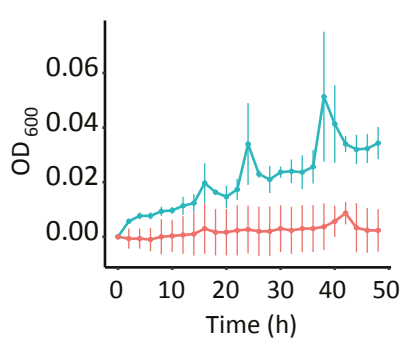

P. corrugata

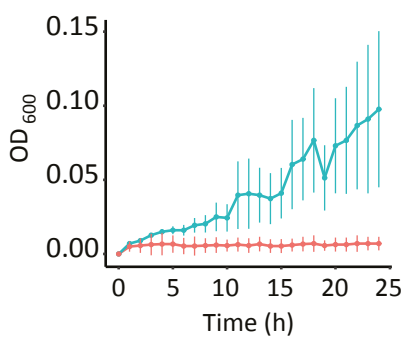

Culture filtrate: $\rightarrow$ - V. dahliae WT $\rightarrow$ - VdAve1::VdAMP2

d

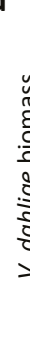

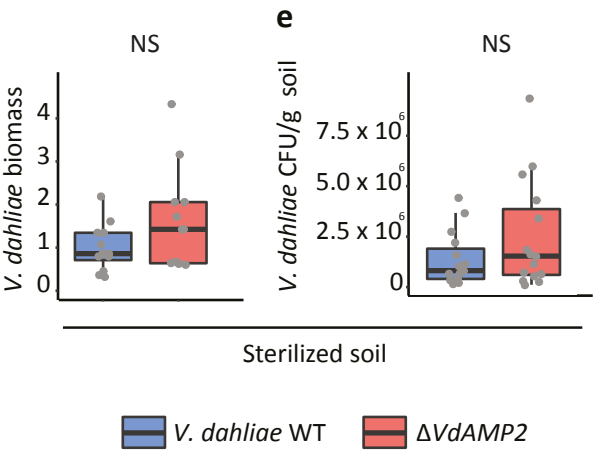

f

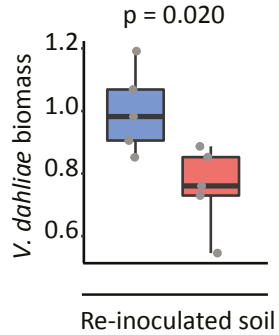

FIGURE 4 | VdAMP2 contributes to Verticillium dahliae soil colonization. (a) V. dahliae VdAMP2 is induced after five days of cultivation in soil extract but not in potato dextrose broth (PDB). The plot displays the average relative expression of three biological replicates \pm SD. (b), Growth of $B$. subtilis and $P$. corrugata in filter-sterilized culture filtrates from wild-type $V$. dahliae and the VdAMP2 expression transformant grown in liquid $0.2 \times \mathrm{PDB}+0.5 \times \mathrm{MS}$ medium. Graphs display the average $\mathrm{OD}_{600}$ of three biological replicates $\pm \mathrm{SD}$. (c), VdAMP2 contributes to soil colonization. $V$. dahliae biomass in soil samples was determined by realtime PCR seven days after inoculation with wild-type $V$. dahliae (WT) and the VdAMP2 deletion mutant ( $\triangle V$ dAMP2) (unpaired two-sided student's t-test; $N=9)$. (d,e) VdAMP2 does not contribute to colonization in sterile soil. Experiment as shown in (c) in sterile soil. $V$. dahliae biomass was quantified with real-time PCR (unpaired two-sided student's t-test; $N=9$ ), (d), and by colony forming unit counts per gram of soil (unpaired two-sided student's t-test; $\mathrm{N}=15)$, (e). (f), Experiment as shown in (d) in sterilized soil supplemented with $10 \%$ fresh potting soil (unpaired two-sided student's t-test; $N=5$ ).

To test for potential antimicrobial activity of VdAMP2, we attempted heterologous production of the effector protein. However, since production in E. coli and Pichia pastoris repeatedly failed, production in $V$. dahliae under control of the VdAve 1 promoter was pursued, resulting in high levels of VdAMP2 expression in vitro (Supplementary Fig. 9b-d). Interestingly, proliferation of B. subtilis and of $P$. corrugata (Fig. 4b), but not of F. oxysporum and of $T$. viride (Supplementary Fig. 9e), was affected by filter-sterilized culture filtrate of the VdAMP2 expression transformant when compared with that of wild-type $V$. dahliae, suggesting that VdAMP2 exerts only antibacterial activity, like VdAve1 although with a different activity spectrum. 
Soil colonization assays using wild-type $V$. dahliae and a VdAMP2 deletion mutant (Supplementary Fig. 9f-h) demonstrated that VdAMP2 contributes to $V$. dahliae fitness in the soil as measured by biomass accumulation (Fig. 4c). Importantly, since this fitness contribution is not observed in sterilized soil, but is regained upon supplementation with fresh potting soil, we conclude that VdAMP2 contributes to $V$. dahliae fitness through its efficacy in microbial competition (Fig. 4d-f). As can be anticipated, the positive effect of VdAMP2 on biomass accumulation in the soil is reflected in disease development when plants are grown on this soil (Supplementary Fig. 10), demonstrating that VdAMP2 positively contributes to virulence of $V$. dahliae in an indirect manner.

\section{Discussion}

Microbial competition occurs in an extremely wide diversity of niches. It is nowadays generally appreciated that a host's microbiome plays a crucial role in its health and, consequently, that hosts actively shape their microbiomes to prevent or suppress disease development. It has also been well established that pathogens secrete effector molecules of various nature during attempted host ingress to promote disease development, many of which target essential components of the host immune system. In our study, we have demonstrated that the fungal broad host-range vascular wilt pathogen $V$. dahliae employs effector proteins that contribute to niche colonization through selective manipulation of local microbiomes, during host-associated as well as during soil-dwelling life stages. Thus, besides the known activities of plant pathogen effector proteins in targeting host physiology, including immune responses, and selfdefense against host-secreted defense molecules, we reveal a novel type of effector activity that involves microbiome manipulation.

A wide array of microbially-secreted molecules has previously been described to fulfill crucial functions in intermicrobial competition, including hydrolytic enzymes, secondary metabolites and antimicrobial proteins. Some Gram-negative bacteria even employ a specialized type VI secretion system (T6SS) to translocate antimicrobial proteins into their microbial competitors ${ }^{90}$. In this manner, Vibrio cholerae, the causal agent of cholera, employs its T6SS to target members of the host commensal microbiota and hereby promotes colonization of the gut ${ }^{91}$. Similarly, the T6SS effector Hyde1 of the phytopathogen Acidovorax citrulli targets plant-associated bacteria in vitro and was speculated to play a role in microbial competition in planta ${ }^{41}$. This T6SS is analogous to the type III secretion system (T3SS) of Gram-negative bacteria that acts as a needle-like structure to directly inject effector proteins into host cells to promote disease ${ }^{92}$. Similar secretion machinery intended for host-microbe or microbe-microbe interactions has not been described for fungi and other filamentous microbes, which instead secrete their effector proteins by extracellular deposition. Consequently, effector molecules 
targeted towards host cells or towards microbial competitors cannot be discriminated based on differential secretion motifs, such as those that determine type III versus type VI secretion in Gram-negatives. Here, we have shown that the pool of effectors secreted by a fungal plant pathogen represents a diverse cocktail comprising proteins involved in the manipulation of the host as well as its microbiome. Consequently, the effectors reported here likely only represent a small proportion of a larger subset of the $V$. dahliae effector repertoire that is intended for microbiome manipulation. For instance, similar effectors might be crucial during advanced infection stages to prevent secondary infections by opportunistic microbes when host defenses are impaired. Additionally, effector proteins can be anticipated to facilitate the survival of the $V$. dahliae resting structures that persist in the microbe-rich soil for years ${ }^{93}$. After all, possibly, fungal effectors with host microbiome-manipulating capacity initially evolved to limit bacterial growth in soil, as the advent of fungi on earth preceded land plant evolution and fungi initially likely co-evolved with bacteria in soil to compete for organic carbon. The discovery of further molecules for microbiome manipulation secreted by $V$. dahliae and other microbes, and unravelling of underlying modes of action, may ultimately lead to the development of novel antibiotics.

\section{Materials and methods}

Xylem fluid isolation. Tomato plants (Solanum lycopersicum cv. Moneymaker) were grown under controlled greenhouse conditions as described previously ${ }^{59}$. The stems of six-week-old plants were cut to allow oozing of the xylem fluid, which was collected on ice with a vacuum pump. The collected xylem fluid was centrifuged for 10 minutes at 20000 x g and filter-sterilized using a $0.2 \mu \mathrm{m}$ filter (Sarstedt, Nümbrecht, Germany). The sterilized xylem fluid was stored at $-20^{\circ} \mathrm{C}$ until use.

Soil extract preparation. To prepare soil extract, 100 grams of dry potting soil (Lentse potgrond, substraat arabidopsis, Lentse Potgrond BV, Katwijk, the Netherlands) was mixed with $500 \mathrm{~mL}$ of demineralized water and autoclaved for 15 minutes at $121^{\circ} \mathrm{C}$. Soil particles were pelleted through centrifugation and the supernatant was collected and stored at $-20^{\circ} \mathrm{C}$ until use.

Gene expression analysis. Total RNA of $V$. dahliae strain JR2 was isolated from tomato roots seven days after root dip inoculation and following five days of in vitro growth in soil extract and potato dextrose broth (PDB) using the Maxwell ${ }^{\oplus} 16$ LEV Plant RNA Kit (Promega, Madison, USA). Real-time PCR was performed as described previously ${ }^{61}$ to determine the expression of effector genes relative to VdGAPDH with primer pairs as shown in Supplementary Table 7.

Production and purification of recombinant effector proteins. The sequences encoding mature VdAve1 and VnAve1 were cloned into pET-15b with an 
$\mathrm{N}$-terminal His 6 tag sequence (Novagen, Madison, WI, USA) (primer sequences, see Supplementary Table 7). The resulting expression vectors were confirmed by sequencing and used to transform E. coli strain BL21. For heterologous protein production, BL21 cells were grown in $1 \times$ YT liquid medium at $37^{\circ} \mathrm{C}$ with constant shaking at $200 \mathrm{rpm}$. Protein production was induced with $1 \mathrm{mM}$ IPTG final concentration when cultures reached an $\mathrm{OD}_{600}=2$ to ensure maximum yields. Following 2 hours of protein production, the bacterial cells were pelleted and snap-frozen in liquid nitrogen and then washed with $100 \mathrm{mM} \mathrm{NaCl}, 1 \mathrm{mM}$ EDTA, and $10 \mathrm{mM}$ Tris at $\mathrm{pH}$ 8.5. Cells were disrupted by stirring for 1 hour in lysis buffer (100 mM Tris, 150 mM NaCl, 10\% glycerol, 6 mg/mL lysozyme (Sigma, St. Louis, MO, USA), $2 \mathrm{mg} / \mathrm{mL}$ deoxycholic acid, $0.06 \mathrm{mg} / \mathrm{mL}$ DNasel, protease inhibitor cocktail (Roche, Mannheim, Germany) at $4^{\circ} \mathrm{C}$. Soluble and insoluble fractions were separated by centrifuging at $20,000 \times g$ for $10 \mathrm{~min}$. The insoluble protein pellets were washed with $10 \mathrm{~mL} 1 \mathrm{M}$ guanidine hydrochloride $(\mathrm{GnHCl}), 10 \mathrm{mM}$ Tris at $\mathrm{pH} 8.0$ and then denatured in $10 \mathrm{~mL} 6 \mathrm{M} \mathrm{GnHCl}, 10 \mathrm{mM} \beta$-mercaptoethanol, $10 \mathrm{mM}$ Tris at $\mathrm{pH}$ 8.0. Samples were incubated for 1 hour at room temperature. Non-denatured debris was pelleted by centrifuging at 20,000 x $g$ for 10 min and discarded. Denaturation was allowed to continue for additional 3-4 hours. Proteins were purified under denaturing conditions by metal affinity chromatography using a column packed with 50\% His60 Ni²+ Superflow Resin (Clontech, Mountain View, CA, USA). The purified effector proteins were dialysed (Spectra/Por ${ }^{\oplus} 3$ Dialysis Membrane, $\mathrm{MWCO}=3.5 \mathrm{kDa}$ ) step-wise against 20 volumes of $0.25 \mathrm{M}$ ammonium sulfate, $0.1 \mathrm{M}$ BisTris, $10 \mathrm{mM}$ reduced glutathione, $2 \mathrm{mM}$ oxidized glutathione, $\mathrm{pH}$ 5.5 with decreasing $\mathrm{GnHCl}$ concentrations for refolding. Each dialysis step was allowed to proceed for at least 24 hours. Finally, proteins were dialysed against demineralized water. Final concentrations were determined using the BioRad Protein Assay (BioRad, Veenendaal, The Netherlands).

Functionality of refolded VdAve1 was confirmed through recognition by the corresponding tomato immune receptor Ve1. To this end, an overnight culture of $A$. tumefaciens strain GV3101 carrying the pSOL2092:Ve1 construct ${ }^{94}$ was harvested by centrifugation and re-suspended to $\mathrm{OD}_{600}=2$ in MMA (2\% sucrose, $0.5 \%$ Murashige \& Skoog salts (Duchefa Biochemie, Haarlem, The Netherlands), 10 mM MES, 200 $\mu \mathrm{M}$ acetosyringone, $\mathrm{pH} 5.6$ ) and infiltrated in the leaves of 5-week-old $N$. tabacum (cv. Petite Havana SR1) plants. After 24 hours, $10 \mu \mathrm{M}$ of purified and refolded $6 x \mathrm{His}-$ VdAve1 was infiltrated in leaf areas expressing Ve1. Photos were taken three days post infiltration of the effector protein.

Generation of $\boldsymbol{V}$. dahliae mutants. To generate the VdAMP2 effector deletion construct, $V d A M P 2$ flanking sequences were amplified using the primers listed in Supplementary Table 7 and cloned into pRF-HU2 ${ }^{95}$. To allow expression of VdAMP2 under control of the $V d A v e 1$ promoter, the coding sequence of $V d A M P 2$ was amplified 
and cloned into pFBT005. All constructs were transformed into $A$. tumefaciens strain AGL1 for V. dahliae transformation as described previously ${ }^{96}$.

V. dahliae culture filtrates. Conidiospores of $V$. dahliae strain JR2 and the VdAMP2 expression transformant were harvested from potato dextrose agar (PDA) and diluted to a final concentration of $10^{4}$ conidiospores $/ \mathrm{mL}$ in $20 \mathrm{~mL}$ of $0.2 x$ PDB supplemented $+0.5 x$ Murashige \& Skoog medium (Duchefa, Haarlem, The Netherlands). Following four days of incubation at $22^{\circ} \mathrm{C}$ and $120 \mathrm{rpm}$, the fungal biomass was pelleted and the remaining supernatants were filter sterilized and stored at $-20^{\circ} \mathrm{C}$ until use.

Bacterial isolates. Bacterial strains Bacillus subtilis AC95, Staphylococcus xylosus M3, Pseudomonas corrugata C26, Streptomyces sp. NE-P-8 and Ralstonia sp. M21 were obtained from our in house endophyte culture collection. Strains used in this study were all isolated from the xylem vessels of tomato cultivars from commercial greenhouses, both from stem and leaf sections. All strains were identified based on their 16S rRNA gene sequence using the primers 27F and 1492R (Supplementary Table 7). 16S amplicons were sequenced by Sanger sequencing at Eurofins (Mix2Seq). The partial $16 \mathrm{~S}$ rRNA gene sequences obtained were evaluated against the $16 \mathrm{~S}$ ribosomal DNA sequence (Bacteria and Archaea) database from NCBI. Bacterial strains Acidovorax sp. (Leaf 73), Arthrobacter sp. (Leaf 69), Rhizobium sp. (Leaf 167), Serratia sp. (Leaf 50), Sphingomonas sp. (Leaf 198), Sphingobium sp. (Leaf 26) and Novosphingobium sp. B (Leaf 2) were obtained from the At-SPHERE collection ${ }^{85}$. Bacterial strains S. mali (DSM 10565) and S. asaccharolytica (DSM 10564) were obtained from the DSMZ culture collection (Braunschweig, Germany). Bacterial strains Novosphingobium sp. A (NCCB 100261), S. macrogoltabida (NCCB 95163), and Sphingobacterium sp. (NCCB 100093) were obtained from the Westerdijk Fungal Biodiversity Institute (Utrecht, The Netherlands).

In vitro microbial growth assays. Bacterial isolates were grown on lysogeny broth agar (LBA) or tryptone soya agar (TSA) at $28^{\circ} \mathrm{C}$. Single colonies were selected and grown overnight at $28^{\circ} \mathrm{C}$ while shaking at $200 \mathrm{rpm}$. Overnight cultures were resuspended to $O D_{600}=0.05$ in xylem fluid supplemented with purified effector proteins or diluted using culture filtrates to $\mathrm{OD}_{600}=0.1$. Additionally, fungal spores were harvested from a PDA plate and suspended in xylem fluid supplemented with purified effector proteins or the $V$. dahliae culture filtrates to a final concentration of $10^{4}$ spores $/ \mathrm{mL}$. $200 \mu \mathrm{L}$ of the microbial suspensions was aliquoted in clear 96 well flat bottom polystyrene tissue culture plates. Plates were incubated in a CLARIOstar ${ }^{\oplus}$ plate reader (BMG LABTECH, Ortenberg, Germany) at $22^{\circ} \mathrm{C}$ with double orbital shaking every 15 minutes (10 seconds at $300 \mathrm{rpm}$ ). The optical density was measured every 15 minutes at $600 \mathrm{~nm}$.

Scanning electron microscopy. Samples for scanning electron microscopy were prepared as described previously with slight modifications ${ }^{97}$. In short, $B$. subtilis strain AC95 was grown overnight in LB and resuspended in xylem fluid to an $\mathrm{OD}_{600}=0.05$. 
Purified effector proteins were added to a final concentration of $6.5 \mu \mathrm{M}(=0.8 \times \mathrm{MIC}$, VdAve1) and bacterial suspensions were incubated for 0, 1, 3 and 7 hours. Next, 20 $\mu \mathrm{L}$ of the bacterial suspensions was transferred to poly-L-lysine coated glass slides (Corning, New York, USA) and incubated for another hour to allow binding of the bacteria. Glass slides were washed using sterile $M Q$ and samples were fixed using $2.5 \%$ glutaraldehyde followed by postfixation in $1 \%$ osmium tetroxide. Samples were dehydrated using an ethanol dehydration series and subjected to critical point drying using a Leica CPD300 (Leica Mikrosysteme GmbH, Vienna, Austria). Finally, the samples were mounted on stubs, coated with $12 \mathrm{~nm}$ of tungsten and visualized in a field emission scanning electron microscope (Magellan 400, FEl, Eindhoven, the Netherlands).

VdAve1 activity assay on cucumber protoplasts. Cucumber protoplasts were obtained by enzymatic digestion of seven-day-old cucumber cotyledons with cellulase and macerozyme, using mannitol as an osmostabilizer. The protoplasts were collected by 1 minute of centrifugation at $100 \times \mathrm{g}$ and carefully resuspended in $1 \mathrm{M}$ sorbitol, 10 $\mathrm{mM}$ MOPS $\mathrm{pH} 6.3$, to a final concentration of $10^{5}-10^{6}$ protoplasts $/ \mathrm{mL}$. Following 30 minutes of incubation, intact protoplasts were quantified for the different treatments using a haemocytometer.

Root microbiome analysis. Tomato and cotton inoculations were performed as described previously ${ }^{59}$. After ten days, plants were carefully uprooted and gently shaken to remove loosely adhering soil from the roots. Next, roots with rhizosphere soil from three tomato or two cotton plants were pooled to form a single biological replicate. Samples were flash-frozen in liquid nitrogen and ground using mortar and pestle. Genomic DNA isolation was performed using the DNeasy PowerSoil Kit (Qiagen, Venlo, The Netherlands). Quality of the DNA samples was checked on a $1.0 \%$ agarose gel. Sequence libraries were prepared following amplification of the V4 region of the bacterial 16S rDNA (515F and 806R), and paired ends (250 bp) were sequenced using the HiSeq2500 sequencing platform (Illumina, San Diego, USA) at the Beijing Genome Institute (BGI, Hong Kong, China).

Sequencing data was processed using R version 3.3.2. as described previously ${ }^{98}$. Briefly, amplicon sequence variants (ASVs) were inferred from quality filtered reads (Phred score $>30$ ) using the DADA2 method ${ }^{99}$. Taxonomy was assigned using the Ribosomal Database Project training set (RDP, version 16) and mitochondria- and chloroplastassigned ASVs were removed. Next, ASV frequencies were transformed according to library size to determine relative abundances. The phyloseq package (version 1.22.3) was used to determine a-diversity (Shannon index) and $\beta$-diversity (BrayCurtis dissimilarity) as described previously ${ }^{98,100}$. Permutational Multivariate Analysis of Variance (PERMANOVA) was performed using the adonis function of vegan package v 2.5-6. Differential abundance analysis was performed using the DESeq2 extension within phylose ${ }^{101}$. To this end, a parametric model was applied to the data and a 
negative binomial Wald test was used to test for differential abundance of bacterial taxa with $p<0.01$ as significance threshold.

Soil colonization assays. Conidiospores of the $V$. dahliae strain JR2 and the mutants were harvested from PDA plate and a total of $10^{6}$ or $10^{7}$ conidiospores were added to $1 \mathrm{gram}$ of potting soil. Samples were incubated at room temperature in the dark for one week. Alternatively, conidiospores were diluted in sterilized (15 minutes at $121^{\circ} \mathrm{C}$ ) and untreated liquid $0.5 \times \mathrm{MS}$ medium supplemented with $3 \%$ sucrose and $2 \%$ potting soil to a final concentration of $10^{6}$ conidiospores $/ \mathrm{ml}$. Samples were incubated at room temperature in the dark for 72 hours Following incubation, DNA was extracted from the samples using the DNeasy PowerSoil Kit (QIAGEN, Venlo, The Netherlands). Microbiome analysis on selected samples was performed as described above. $V$. dahliae biomass was quantified through real-time PCR using $V$. dahliae specific primers targeting the internal transcribed spacer (ITS) region of the ribosomal DNA (Supplementary Table 7). Primers targeting a conserved region of the bacterial 16S rRNA gene were used for sample equilibration.

To allow sample calibration when using sterilized potting soil or MS medium (15 minutes at $121^{\circ} \mathrm{C}$ ), the same amount of fresh potting soil was added to the samples prior to DNA extraction. Additionally, after one week of incubation of $V$. dahliae in the sterilized soil, serial dilutions were made and plated onto PDA to quantify colony forming units.

Treatment of synthetic community with purified VdAve1. A pre-mixed synthetic community comprising a total of 137 bacterial strains from the At-SPHERE collection $^{85}$ was diluted in liquid R2A medium supplemented with demineralized water or $4 \mu \mathrm{MVdAve} 1$ to a concentration of $\mathrm{OD}_{600}=0.002$. The bacterial suspensions were grown overnight at $22^{\circ} \mathrm{C}$ with constant shaking at $120 \mathrm{rpm}$. Next, bacterial cells were pelleted and DNA was extracted from the samples using the DNeasy PowerSoil Kit (QIAGEN, Venlo, The Netherlands). Microbiome analysis was performed as described above using $\mathrm{p}$ adjusted $<0.05$ as significance threshold for the differential abundance analysis.

In vitro competition assay. Conidiospores of V. dahliae strain JR2 and the VdAve 1 deletion and complementation mutants were harvested from a PDA plate using sterile water and diluted to a final concentration of $10^{6}$ conidiospores $/ \mathrm{mL}$ in liquid 0.5x MS (Murashige and Skoog) medium (Duchefa, Haarlem, The Netherlands). Next, overnight cultures of the bacterial isolates were added to the conidiospores to $\mathrm{OD}_{600}=0.05$ and $500 \mu \mathrm{L}$ of the microbial suspensions was aliquoted in clear 12well flat-bottom polystyrene tissue culture plates. Following 48 hours of incubation at room temperature, the microbial cultures were recovered and genomic DNA was isolated using the SmartExtract - DNA Extraction Kit (Eurogentec, Maastricht, The Netherlands). V. dahliae biomass was quantified through real-time PCR using $V$. 
dahliae specific primers targeting the internal transcribed spacer (ITS) region of the ribosomal DNA (Supplementary Table 7).

In planta competition assay. To allow S. macrogoltabida and Ralstonia sp. colonization of in the absence of other microbes, tomato seeds were incubated for five minutes in $2 \%$ sodium hypochlorite to ensure surface sterilization. Next, surface sterilized tomato seeds were washed three times using sterile water and transferred to a sterile Petri dish containing a filter paper pre-moistened with a S. macrogoltabida or Ralstonia sp. suspension in water $\left(\mathrm{OD}_{600}=0.05\right)$. The tomato seeds were allowed to germinate in vitro and eventually transferred to regular potting soil, ten-day-old seedlings were inoculated as described previously ${ }^{59}$. Tomato stems were collected at 14 days post inoculation (dpi) and lyophilized prior to genomic DNA isolation with a CTAB-based extraction buffer (100 mM Tris- $\mathrm{HCl}$ pH 8.0, 20 mM EDTA, $2 \mathrm{M} \mathrm{NaCl}$, $3 \%$ CTAB). V. dahliae biomass was quantified with real-time PCR on the genomic DNA by targeting the internal transcribed spacer (ITS) region of the ribosomal DNA. The tomato rubisco gene was used for sample calibration. S. macrogoltabida biomass was quantified using Sphingopyxis specific primers (Supplementary Table 7 ) and normalized using the V. dahliae ITS. Additionally, the relative abundance of the Sphingomonadales in three representative samples was determined by $16 \mathrm{~S}$ ribosomal DNA profiling as described previously.

Disease assays using $\boldsymbol{V}$. dahliae microsclerotia. $V$. dahliae microsclerotia were produced in a sterile moist medium of vermiculite and maize meal as described previously ${ }^{102}$. After four weeks of incubation, the vermiculite/microsclerotia mixture was dried at room temperature. Next, $150 \mathrm{~mL}$ of the dried mixture was mixed with $1 \mathrm{~L}$ of potting soil (Lentse potgrond, substraat arabidopsis, Lentse Potgrond BV, Katwijk The Netherlands) and Arabidopsis seeds of the Col-0 ecotype were sown at equal distances on top of the mixture. The above-ground parts of the plants were collected at $27 \mathrm{dpi}$ and $V$. dahliae biomass was quantified through real-time PCR using $V$. dahliae specific primers targeting the internal transcribed spacer (ITS) region of the ribosomal DNA. The Arabidopsis rubisco gene was used for sample calibration (Supplementary Table 7). 


\section{Acknowledgements}

The authors thank M. Giesbers from the Wageningen Electron Microscopy Centre for technical assistance. Work in the laboratory of B.P.H.J.T. is supported by the Research Council Earth and Life Sciences (ALW) of the Netherlands Organization of Scientific Research (NWO). BPHJT acknowledges support by the Deutsche Forschungsgemeinschaft (DFG, German Research Foundation) under Germany's Excellence Strategy - EXC 2048/1 - Project ID: 390686111.

\section{Author contributions}

N.C.S., H.R. and B.P.H.J.T. conceived the project. N.C.S., H.R., G.C.P., J.R.M. and B.P.H.J.T. designed the experiments. N.C.S., H.R., G.C.P., M.R.F., G.C.M.B., M.F.S. and R.N. carried out the experiments, N.C.S., H.R., G.C.P., M.R.F., M.F.S., J.A.V., R.N., J.R.M. and B.P.H.J.T. analyzed the data. N.C.S. and B.P.H.J.T. wrote the manuscript. All authors read and approved the final manuscript.

\section{Data and materials availability}

The metagenomics data have been deposited in the European Nucleotide Archive (ENA) under accession number PRJEB34281. 


\section{Supplementary data}

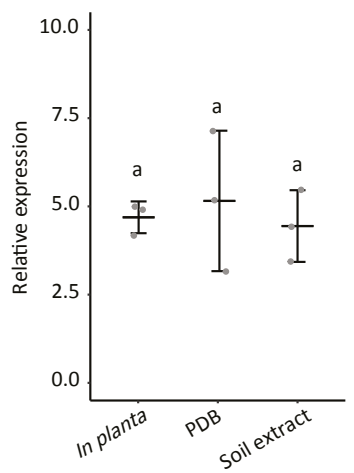

SUPPLEMENTARY FIGURE 1 | The VdAve1 effector gene is ubiquitously expressed by Verticillium dahliae. VdAve1 is among the most highly expressed effector genes in planta ${ }^{61,62,81}$. The graph displays expression of $V d A v e 1$ relative to $V d G A P D H$ during colonization of tomato roots at 7 days post inoculation, growth in potato dextrose broth (PDB) at 5 days of cultivation, or growth in soil extract at 5 days of cultivation. The plot displays the average expression of three biological replicates \pm SD. Letter labels indicate nonsignificant differences (one-way ANOVA and Tukey's post-hoc test; $p<0.05$ ). 


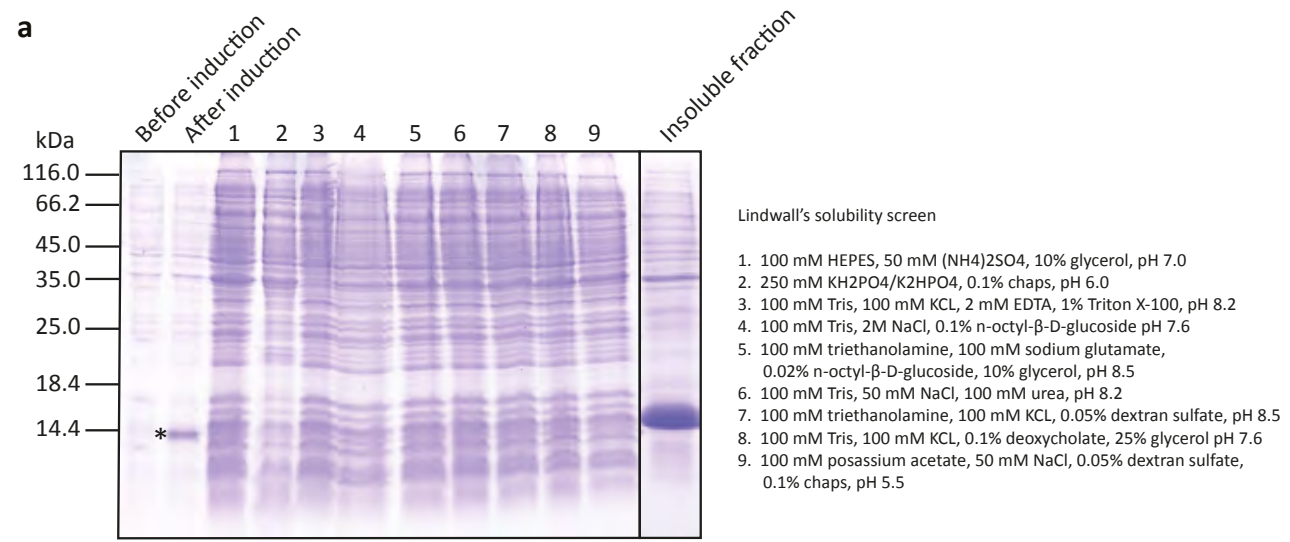

b

$35 \mathrm{~s}:: \mathrm{Ve1}+$

$35 s:: V e 1$

$10 \mu \mathrm{M}$ 6xHis-VdAve

$10 \mu \mathrm{M}$ 6xHis-VdAve1
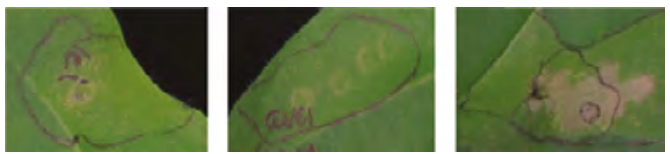

SUPPLEMENTARY FIGURE 2 | Heterologously produced VdAve1 can be isolated from inclusion bodies. (a) E. coli BL21 cells were grown in liquid YT medium and VdAve1 expression was induced using $1 \mathrm{mM}$ IPTG. Following four hours of protein production at $28^{\circ} \mathrm{C}$, the presence of $\mathrm{VdAve} 1$ was confirmed by boiling the cells in $1 \%$ SDS, $2 \mathrm{M}$ urea, $1.25 \% \beta$-mercaptoethanol, $2.5 \%$ glycerol, $15 \mathrm{mM}$ Tris, $\mathrm{pH}$ 6.8. The band representing VdAve1 is indicated with an asterisk; limited solubility of the protein was detected upon sonication of the cells in the corresponding buffers (lanes 1-9). The presence of VdAve1 in the insoluble protein fractions was confirmed following denaturation of the insoluble proteins, indicating the formation of inclusion bodies. (b) VdAve1 purified from the insoluble protein fraction under denaturing conditions was refolded by step-wise dialysis. Functionality of the protein was confirmed by infiltration into $N$. tabacum leaf sections overexpressing the corresponding tomato immune receptor Ve1, resulting in a hypersensitive response at three days post infiltration. 
a
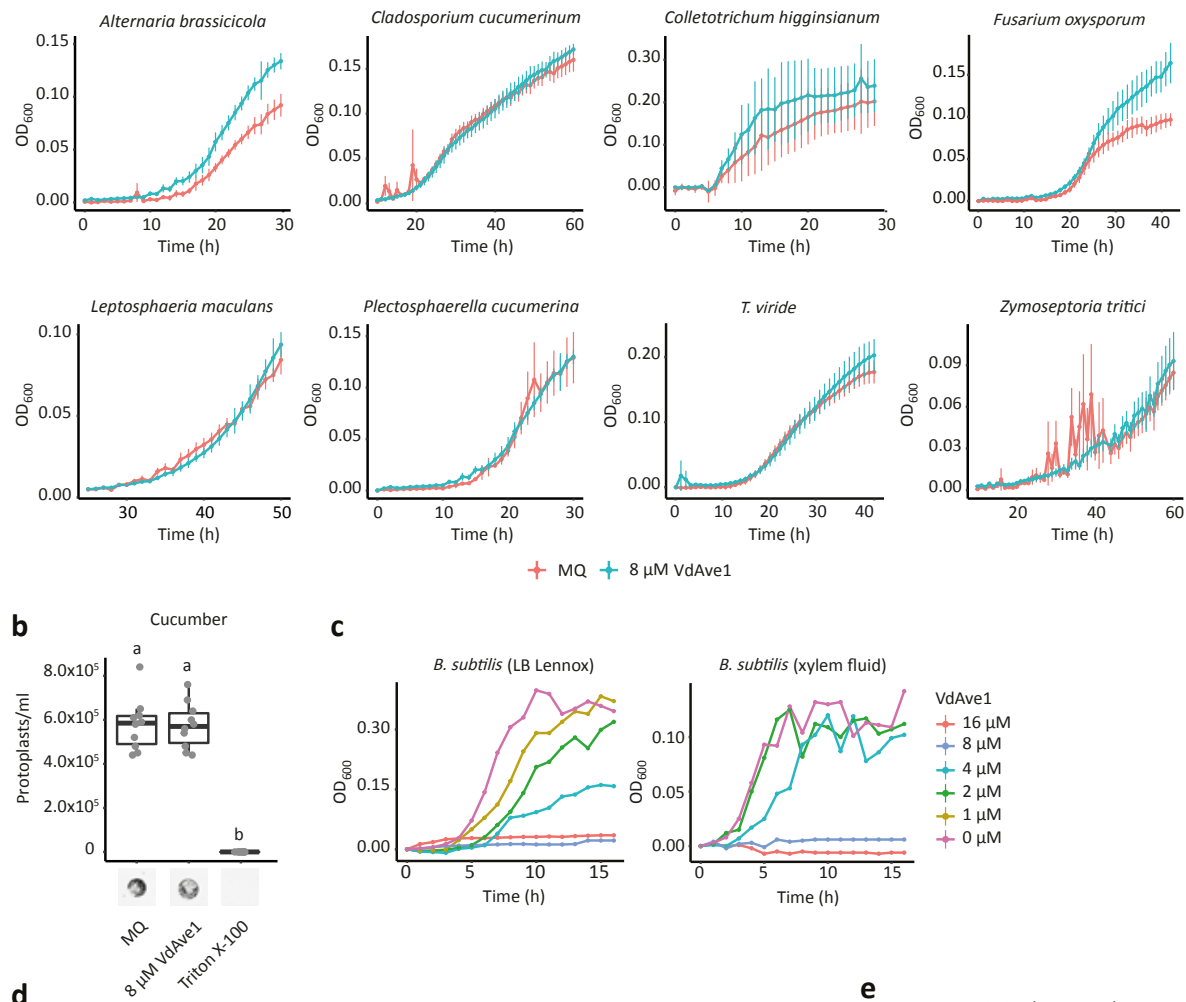

C
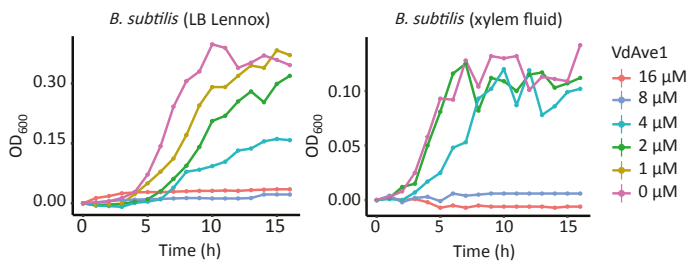

d
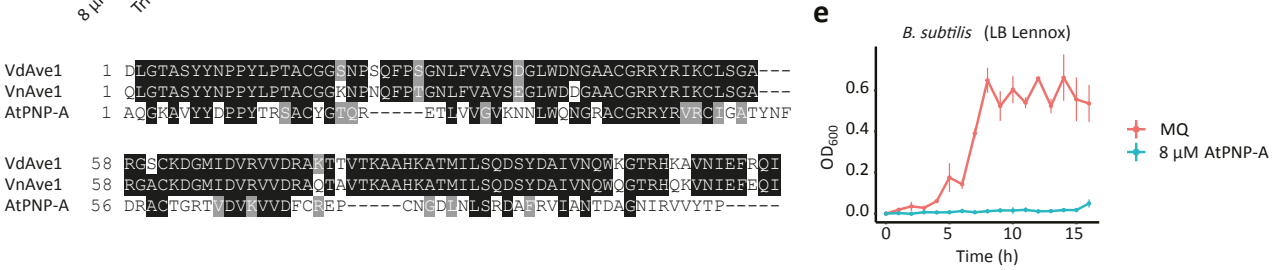

SUPPLEMENTARY FIGURE 3 | VdAve1 displays antibacterial, but not antifungal or phytotoxic, activity. (a) In vitro growth of fungal species in tomato xylem fluid is not inhibited by $8 \mu \mathrm{M}$ VdAve1. Graphs display the average $\mathrm{OD}_{600}$ of three biological replicates $\pm \mathrm{SD}$. (b) VdAve1 does not affect plant protoplasts. Cucumber protoplasts were incubated with $8 \mu \mathrm{M}$ VdAve1. After 30 minutes, the number of protoplasts was quantified using a haemocytometer. Triton $X-100$ and $M Q$ were included as positive and negative control for protoplast disruption, respectively. Representative phenotypes of the protoplasts under the various experimental conditions are displayed under the boxplots (one-way ANOVA and Tukey's post-hoc test; $\mathrm{p}<0.05 ; \mathrm{N}=10$ ). (c) Determination of the minimum inhibitory concentration of VdAve1 on B. subtilis upon overnight incubation in LB Lennox and tomato xylem fluid. No growth was detected upon incubation with 8 $\mu \mathrm{MVdAve} 1$ or more. (d) Multiple sequence alignment of mature VdAve1 with the homologs from V. nubilum and $A$. thaliana. (e) In vitro growth of $B$. subtilis is inhibited by $8 \mu M$ AtPNP-A. Graphs display the average $\mathrm{OD}_{600}$ of three biological replicates $\pm \mathrm{SD}$. 
A
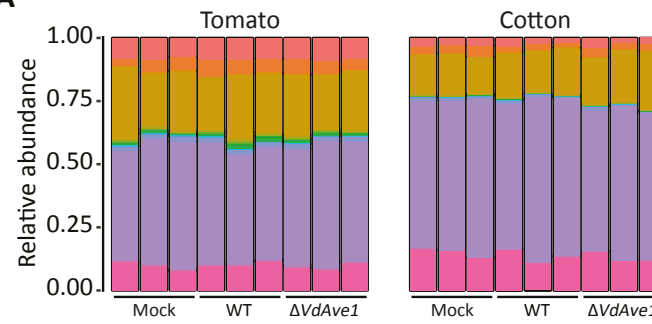

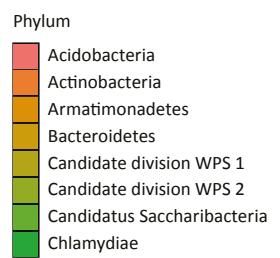

C
B

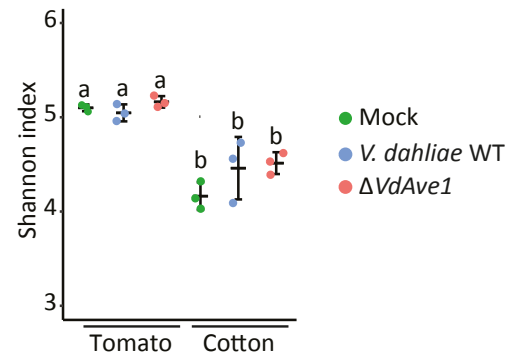

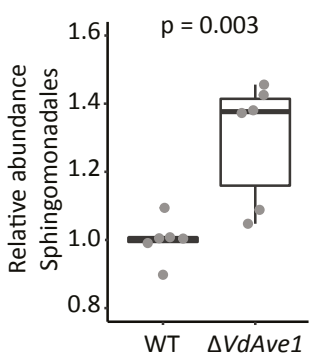

SUPPLEMENTARY FIGURE 4 | Metagenomic characterization of tomato and cotton root microbiomes upon Verticillium dahliae infection. (a) Relative abundance of bacterial phyla in the root microbiomes of tomato and cotton plants ten days after inoculation with wild-type $V$. dahliae (WT) and a VdAve 1 deletion mutant as determined by $16 \mathrm{~S}$ ribosomal DNA profiling. (b) $V$. dahliae inoculation does not change a-diversity of host root microbiomes (one-way ANOVA and Tukey's post-hoc test; $\mathrm{p}<0.05 ; \mathrm{N}=3$ ). The plot displays the average Shannon index \pm SD. (c) Sphingomonadales are significantly enriched in the microbiomes of roots that are colonized by the VdAve 1 deletion mutant. Differential abundance analysis of bacterial orders following combination of tomato and cotton samples based on infection by the different $V$. dahliae genotypes, only revealed the Sphingomonadales as differentially abundant (unpaired two-sided student's t-test, $p<0.01 ; N=6$ ). Relative abundances were normalized against the average relative abundance upon infection of the corresponding host by wild-type $V$. dahliae to correct for host-dependent differences. 
a
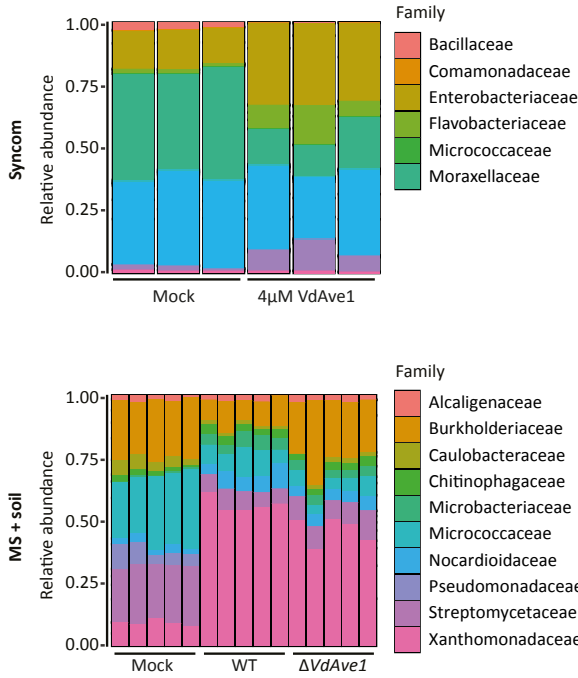

Family
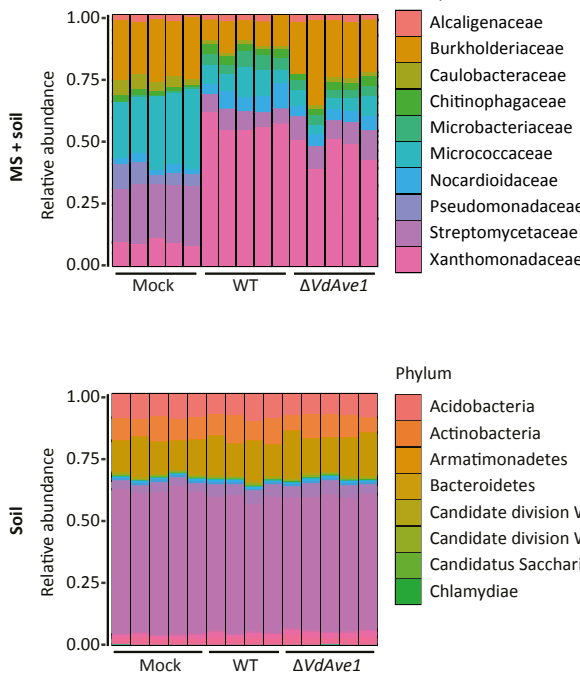

Phylum

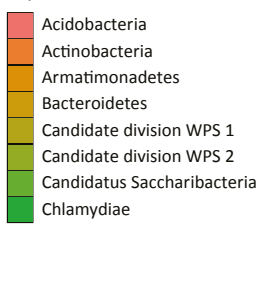

b
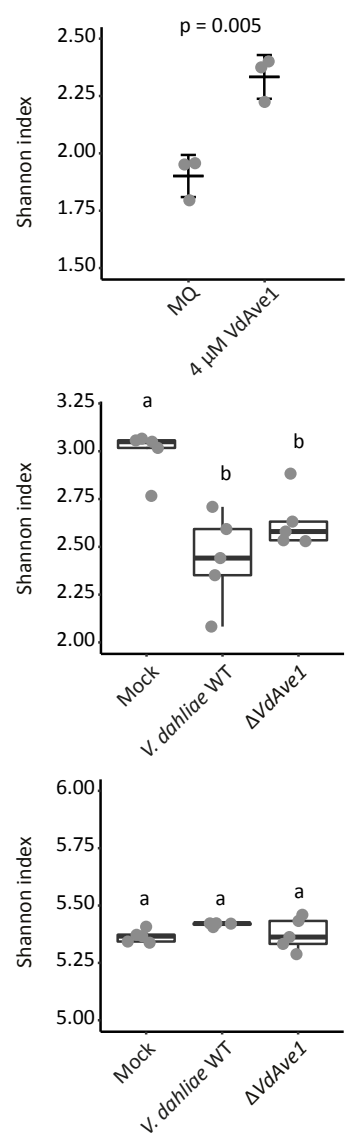

SUPPLEMENTARY FIGURE 5 | Metagenomic characterization of soil microbiomes and a synthetic community upon Verticillium dahliae inoculation or VdAve1 treatment. (a) Relative abundance of bacterial phyla or families in a synthetic community (Syncom), in MS medium supplemented with soil, and in soil after inoculation with wild-type $V$. dahliae (WT), a VdAve1 deletion strain, or treatment with purified VdAve1. (b) Impact of $V$. dahliae inoculation or $V d A v e 1$ treatment on a-diversity in the microbiomes as shown in (a) (unpaired two-sided student's t-test; $N=3$ ) (one-way ANOVA and Tukey's post-hoc test; $p<0.05$; $\mathrm{N} \geq 4$ ). The plot for the syncom displays the average Shannon index \pm SD. 
a
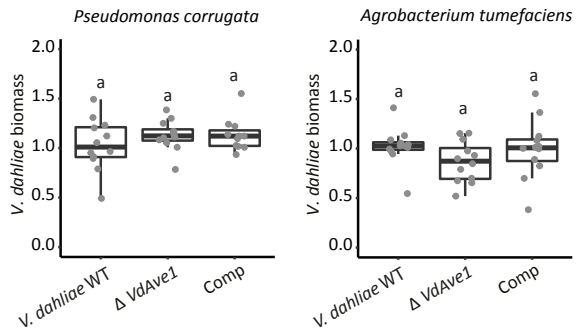

b
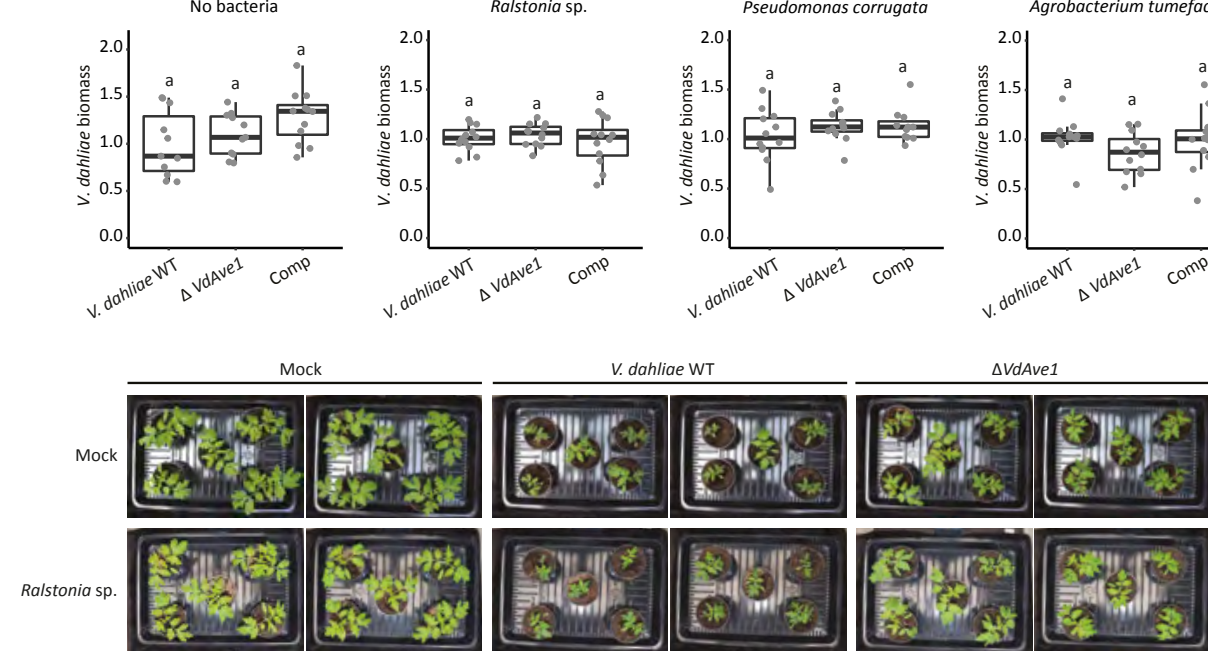

c

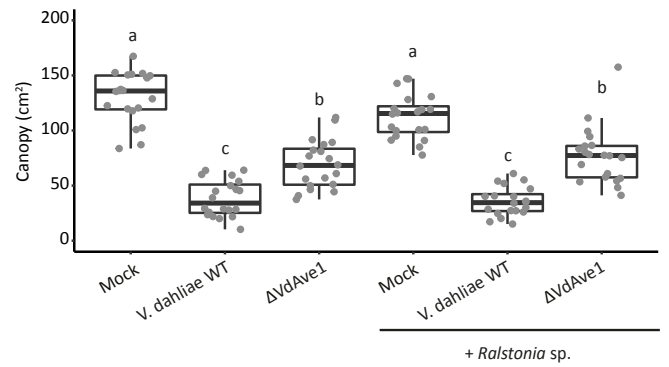

d

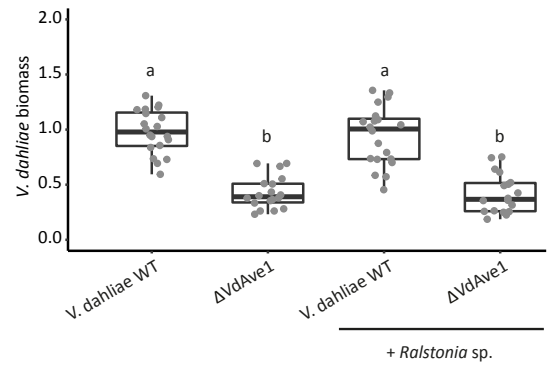

SUPPLEMENTARY FIGURE 6 | In vitro and in planta competition assays of $V$. dahliae with VdAve1insensitive bacterial isolates. (a) VdAve 1 does not contribute to $V$. dahliae colonization in vitro in the presence of Ralstonia sp., P. corrugata or A. tumefaciens. Letters represent non-significant biomass differences (one-way ANOVA and Tukey's post-hoc test; $\mathrm{p}<0.05 ; \mathrm{N}=12$ ). (b) Tomato seed treatment with Ralstonia sp. does not reduce Verticillium wilt symptoms. Phenotypes of tomato plants 14 days post inoculation with wild-type $V$. dahliae or the $V d A v e 1$ deletion mutant. Tomato seeds were surface-sterilized and allowed to germinate in vitro in the presence or the absence of Ralstonia sp. prior to infection. (c) Canopy area of mock-treated and Ralstonia-treated tomato plants infected by wild-type $V$. dahliae or the VdAve 1 deletion mutant (one-way ANOVA and Tukey's post-hoc test; $\mathrm{p}<0.05 ; \mathrm{N}=20$ ). (d) V. dahliae biomass in tomato stems determined with real-time PCR. Letters represent significant biomass differences (one-way ANOVA and Tukey's post-hoc test; $\mathrm{p}<0.05 ; \mathrm{N} \geq 18$ ). 
a

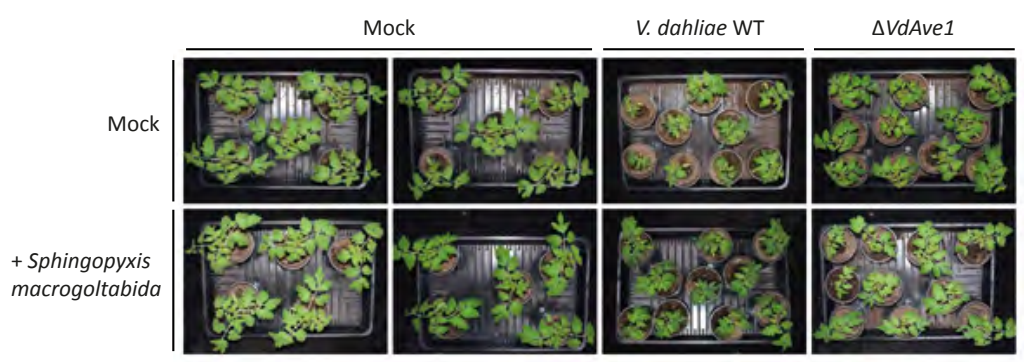

b

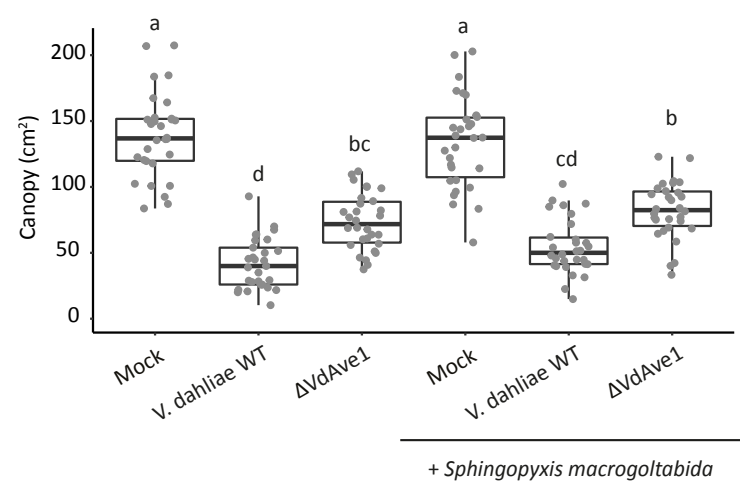

SUPPLEMENTARY FIGURE 7 | Tomato seed treatment with S. macrogoltabida reduces Verticillium wilt symptoms. (a) Phenotypes of tomato plants 14 days post inoculation with wild-type $V$. dahliae or the VdAve1 deletion mutant. Tomato seeds were surface-sterilized and allowed to germinate in vitro in the presence or the absence of S. macrogoltabida prior to infection. (b) Canopy area of mock-treated and S. macrogoltabida-treated tomato plants infected by wild-type $V$. dahliae or the VdAve1 deletion mutant. Letters represent significant differences (one-way ANOVA and Tukey's post-hoc test; $p<0.01 ; N \geq 29$ ). 


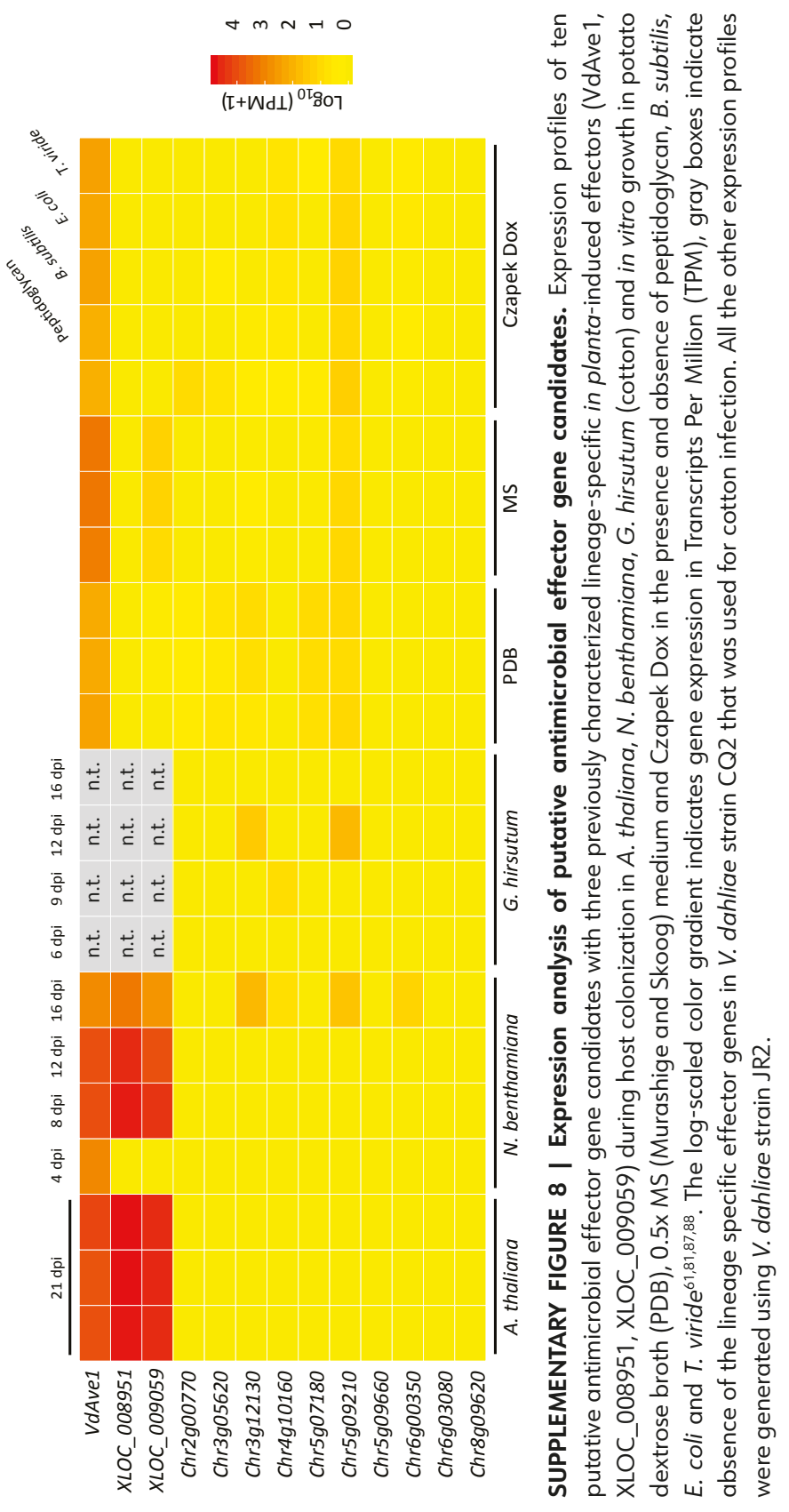


a

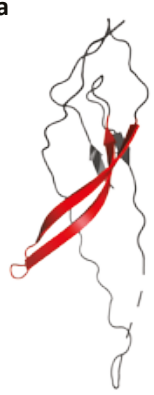

b

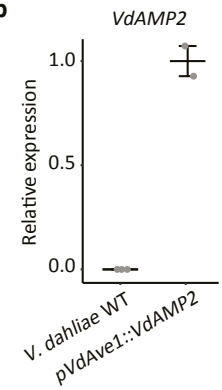

c

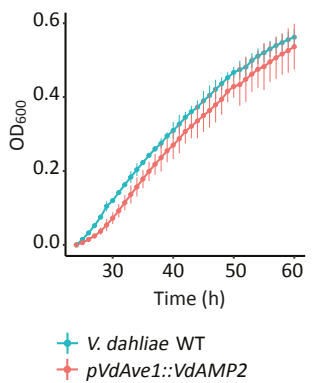

d

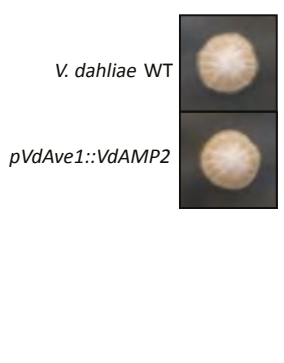

e
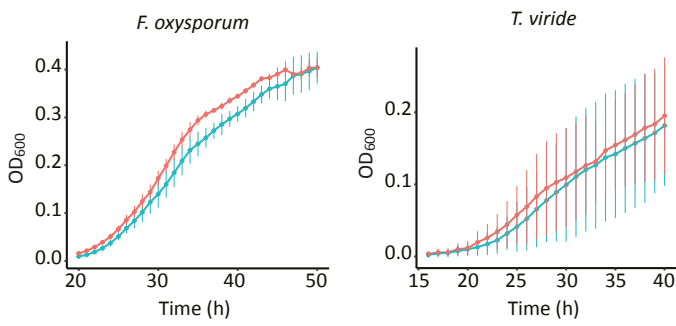

Culture filtrate: $-\downarrow-$ V. dahliae WT $\rightarrow-p$ VdAve1::VdAMP2

f

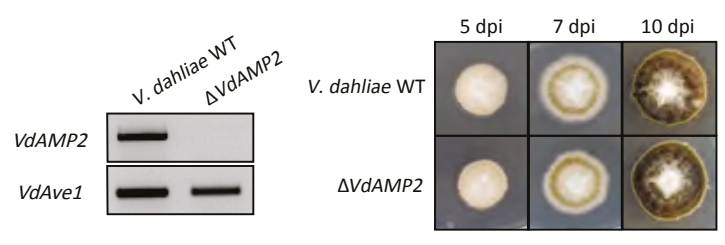

h

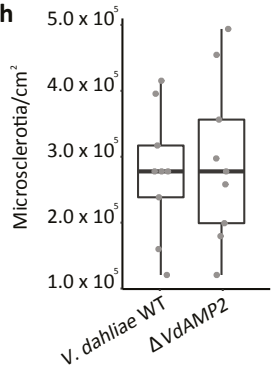

SUPPLEMENTARY FIGURE 9 | VdAMP2 structure, antifungal activity assays and $\boldsymbol{V}$. dahliae mutants. (a) VdAMP2 shares structural homology with the amphipathic $\beta$-hairpins of aerolysin-type $\beta$-pore forming toxins. Predicted protein structure of part of VdAMP2 (aa 225-312) with the amphipathic $\beta$-hairpin highlighted in red. Protein structure was predicted using Phyre ${ }^{103}$. (b) In vitro expression of VdAMP2 in wildtype $V$. dahliae and the $p V d A v e 1:: V d A M P 2$ mutant after five days of cultivation in liquid $0.5 x$ Murashige \& Skoog (MS) medium. Plot displays the average expression \pm SD. (c-d) In vitro expression of VdAMP2 does not affect $V$. dahliae growth. $c$, Growth of wild-type $V$. dahliae and the $p$ VdAve1::VdAMP2 mutant in liquid $0.2 x$ potato dextrose broth (PDB) $+0.5 \times \mathrm{MS}$. Graphs display the average $\mathrm{OD}_{600}$ of three biological replicates \pm SD. (d) Morphology of wild-type $V$. dahliae and the $p V d A v e 1:: V d A M P 2$ mutant after five days of cultivation on potato dextrose agar (PDA). (e) VdAMP2 does not inhibit fungal growth. Growth of $F$. oxysporum and $T$. viride in filter-sterilized culture filtrates from in vitro grown wild-type $V$. dahliae and the VdAMP2 expression transformant does not reveal antifungal activity of VdAMP2. Graphs display the average $\mathrm{OD}_{600}$ of three biological replicates $\pm S D$. (f) Deletion of $V d A M P 2$ was confirmed using PCR on genomic DNA of wild-type $V$. dahliae and the VdAMP2 deletion mutant ( $\triangle V d A M P 2)$, VdAve 1 was used as genomic DNA control. (g) Morphology of wild-type V. dahliae and the VdAMP2 deletion mutant at five, seven and ten days of cultivation on PDA. (h) The VdAMP2 deletion mutant is not affected in microsclerotia formation ( $N=9$ ). After ten days, colonies as shown in (f) were excised from plate and the tissue was ground to determine the number of microsclerotia per $\mathrm{cm}^{2}$ using a haemocytometer. 


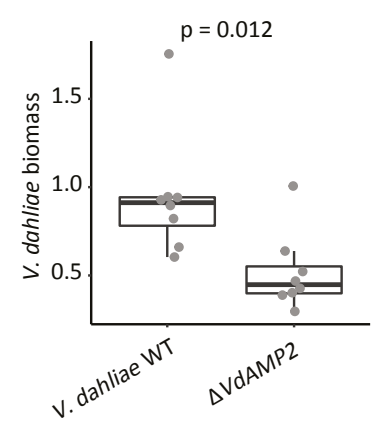

SUPPLEMENTARY FIGURE 10 | VdAMP2 contributes to host colonization. Biomass of wild-type V. dahliae and the VdAMP2 deletion mutant $(\triangle V D A M P 2)$ in $A$. thaliana plants determined with real-time PCR at 27 days after sowing the seeds on soil containing microsclerotia of $V$. dahliae (unpaired two-sided student's $\mathrm{t}$-test; $\mathrm{N}=8$ ). 
SUPPLEMENTARY TABLE 1 | DESeq2 output of differential abundance analysis of bacterial orders in tomato root microbiomes colonized by wild-type $V$. dahliae and the VdAve1 deletion mutant.

\begin{tabular}{|c|c|c|c|c|c|}
\hline Base mean & $\log 2 \mathrm{FC}$ & IfcSE & stat & p value & Order \\
\hline 340.07 & 0.75 & 0.21 & 3.56 & $3.67 \mathrm{E}-04$ & Bdellovibrionales \\
\hline 265.53 & -0.60 & 0.19 & -3.24 & $1.18 \mathrm{E}-03$ & Sphingomonadales \\
\hline 143.41 & 0.97 & 0.31 & 3.17 & $1.55 \mathrm{E}-03$ & Ktedonobacterales \\
\hline 2126.65 & -0.32 & 0.12 & -2.57 & $1.02 \mathrm{E}-02$ & Xanthomonadales \\
\hline 146.92 & 1.30 & 0.58 & 2.25 & $2.42 \mathrm{E}-02$ & Pseudomonadales \\
\hline 705.03 & -0.32 & 0.15 & -2.17 & $3.01 \mathrm{E}-02$ & Alphaproteobacteria incertae sedis \\
\hline 104.59 & 0.70 & 0.34 & 2.06 & 3.97E-02 & Chlamydiales \\
\hline 5.72 & -5.79 & 2.87 & -2.01 & 4.39E-02 & Acidimicrobiales \\
\hline 28.94 & 1.95 & 0.98 & 2.00 & $4.54 \mathrm{E}-02$ & Granulicella \\
\hline 27.37 & -1.21 & 0.90 & -1.34 & $1.80 \mathrm{E}-01$ & Hydrogenophilales \\
\hline 444.45 & 0.24 & 0.18 & 1.34 & $1.80 \mathrm{E}-01$ & Cytophagales \\
\hline 25.12 & -1.00 & 0.74 & -1.34 & $1.81 \mathrm{E}-01$ & Fimbriimonadales \\
\hline 2.89 & -4.81 & 3.59 & -1.34 & $1.81 \mathrm{E}-01$ & Sulfuricellales \\
\hline 2.45 & 4.92 & 3.72 & 1.32 & $1.86 \mathrm{E}-01$ & Methanosarcinales \\
\hline 960.84 & 0.18 & 0.16 & 1.18 & $2.37 \mathrm{E}-01$ & Actinomycetales \\
\hline 308.73 & -0.21 & 0.19 & -1.10 & $2.72 \mathrm{E}-01$ & Caulobacterales \\
\hline 150.02 & -0.36 & 0.33 & -1.09 & $2.76 \mathrm{E}-01$ & Spirochaetales \\
\hline 442.03 & 0.17 & 0.17 & 1.04 & $3.00 \mathrm{E}-01$ & Myxococcales \\
\hline 1.74 & -4.07 & 3.93 & -1.04 & $3.00 \mathrm{E}-01$ & Aeromonadales \\
\hline 574.02 & -0.19 & 0.18 & -1.03 & $3.01 \mathrm{E}-01$ & Flavobacteriales \\
\hline 2112.11 & -0.09 & 0.09 & -1.03 & $3.04 \mathrm{E}-01$ & Burkholderiales \\
\hline 1.65 & -3.99 & 3.93 & -1.02 & $3.10 \mathrm{E}-01$ & Thermoanaerobacterales \\
\hline 1.48 & -3.84 & 3.93 & -0.98 & $3.29 \mathrm{E}-01$ & Gammaproteobacteria incertae sedis \\
\hline 1.14 & 3.82 & 3.95 & 0.97 & 3.34E-01 & Desulfuromonadales \\
\hline 1.09 & 3.75 & 3.95 & 0.95 & $3.43 \mathrm{E}-01$ & Legionellales \\
\hline 37.82 & 0.50 & 0.55 & 0.91 & $3.65 \mathrm{E}-01$ & Gaiellales \\
\hline 301.06 & -0.17 & 0.20 & -0.85 & 3.97E-01 & Rhodospirillales \\
\hline 160.75 & -0.20 & 0.24 & -0.81 & 4.17E-01 & Gp3 \\
\hline 27.70 & -0.62 & 0.79 & -0.79 & 4.27E-01 & Bacteroidales \\
\hline 19.95 & -0.68 & 0.86 & -0.79 & 4.32E-01 & Clostridiales \\
\hline 2559.50 & -0.08 & 0.10 & -0.77 & 4.39E-01 & Sphingobacteriales \\
\hline 1322.35 & 0.09 & 0.12 & 0.76 & 4.45E-01 & Rhizobiales \\
\hline 14.69 & -2.52 & 3.33 & -0.76 & 4.49E-01 & Acidipila \\
\hline 990.27 & -0.08 & 0.12 & -0.68 & 4.99E-01 & Telmatobacter \\
\hline 6.39 & -1.42 & 2.24 & -0.63 & $5.26 \mathrm{E}-01$ & Enterobacteriales \\
\hline 23.72 & 0.50 & 0.80 & 0.62 & 5.35E-01 & Holophagales \\
\hline 0.44 & 2.41 & 4.02 & 0.60 & $5.49 \mathrm{E}-01$ & Sneathiellales \\
\hline 3.16 & -1.93 & 3.23 & -0.60 & $5.51 \mathrm{E}-01$ & Nitrososphaeraceae \\
\hline 9.56 & -1.29 & 2.19 & -0.59 & 5.57E-01 & Candidatus_Koribacter \\
\hline 270.02 & 0.12 & 0.21 & 0.56 & $5.76 \mathrm{E}-01$ & Verrucomicrobiales \\
\hline
\end{tabular}




\begin{tabular}{llllll}
\hline Base mean & log2FC & IfcSE & stat & p value & Order \\
\hline 4.54 & -1.57 & 2.87 & -0.55 & $5.85 \mathrm{E}-01$ & Tepidisphaerales \\
\hline 120.32 & 0.16 & 0.34 & 0.47 & $6.39 \mathrm{E}-01$ & Terriglobus \\
\hline 76.20 & -0.16 & 0.41 & -0.39 & $6.94 \mathrm{E}-01$ & Solirubrobacterales \\
\hline 66.75 & 0.14 & 0.37 & 0.37 & $7.10 \mathrm{E}-01$ & Gallionellales \\
\hline 53.14 & -0.55 & 1.49 & -0.37 & $7.12 \mathrm{E}-01$ & Acidobacterium \\
\hline 2.86 & 1.31 & 3.68 & 0.36 & $7.22 \mathrm{E}-01$ & Gp2 \\
\hline 37.32 & -0.15 & 0.52 & -0.30 & $7.68 \mathrm{E}-01$ & Armatimonadales \\
\hline 2.07 & 0.88 & 3.51 & 0.25 & $8.03 \mathrm{E}-01$ & Lactobacillales \\
\hline 11.06 & -0.45 & 1.82 & -0.25 & $8.05 \mathrm{E}-01$ & Gemmatimonadales \\
\hdashline 1.79 & 0.80 & 3.83 & 0.21 & $8.34 \mathrm{E}-01$ & Anaerolineales \\
\hline 372.06 & -0.04 & 0.18 & -0.21 & $8.36 \mathrm{E}-01$ & Planctomycetales \\
\hline 1.93 & 0.54 & 3.82 & 0.14 & $8.88 \mathrm{E}-01$ & Chthonomonadales \\
\hline 72.62 & -0.06 & 0.48 & -0.13 & $8.94 \mathrm{E}-01$ & Opitutales \\
\hline 14.47 & -0.19 & 1.60 & -0.12 & $9.07 \mathrm{E}-01$ & Bacillales \\
\hline 115.79 & -0.04 & 0.35 & -0.11 & $9.10 \mathrm{E}-01$ & Rhodocyclales \\
\hdashline 118.47 & 0.02 & 0.28 & 0.08 & $9.34 \mathrm{E}-01$ & Gp1 \\
\hline 72.58 & -0.02 & 0.37 & -0.06 & $9.56 \mathrm{E}-01$ & Candidatus_Solibacter \\
\hline 12.39 & 0.08 & 1.47 & 0.05 & $9.58 \mathrm{E}-01$ & Nitrosomonadales \\
\hdashline 11.43 & -0.05 & 1.27 & -0.04 & $9.69 \mathrm{E}-01$ & Terrimicrobium \\
\hdashline 224.78 & 0.01 & 0.76 & 0.01 & $9.94 \mathrm{E}-01$ & Chromatiales \\
\hline
\end{tabular}




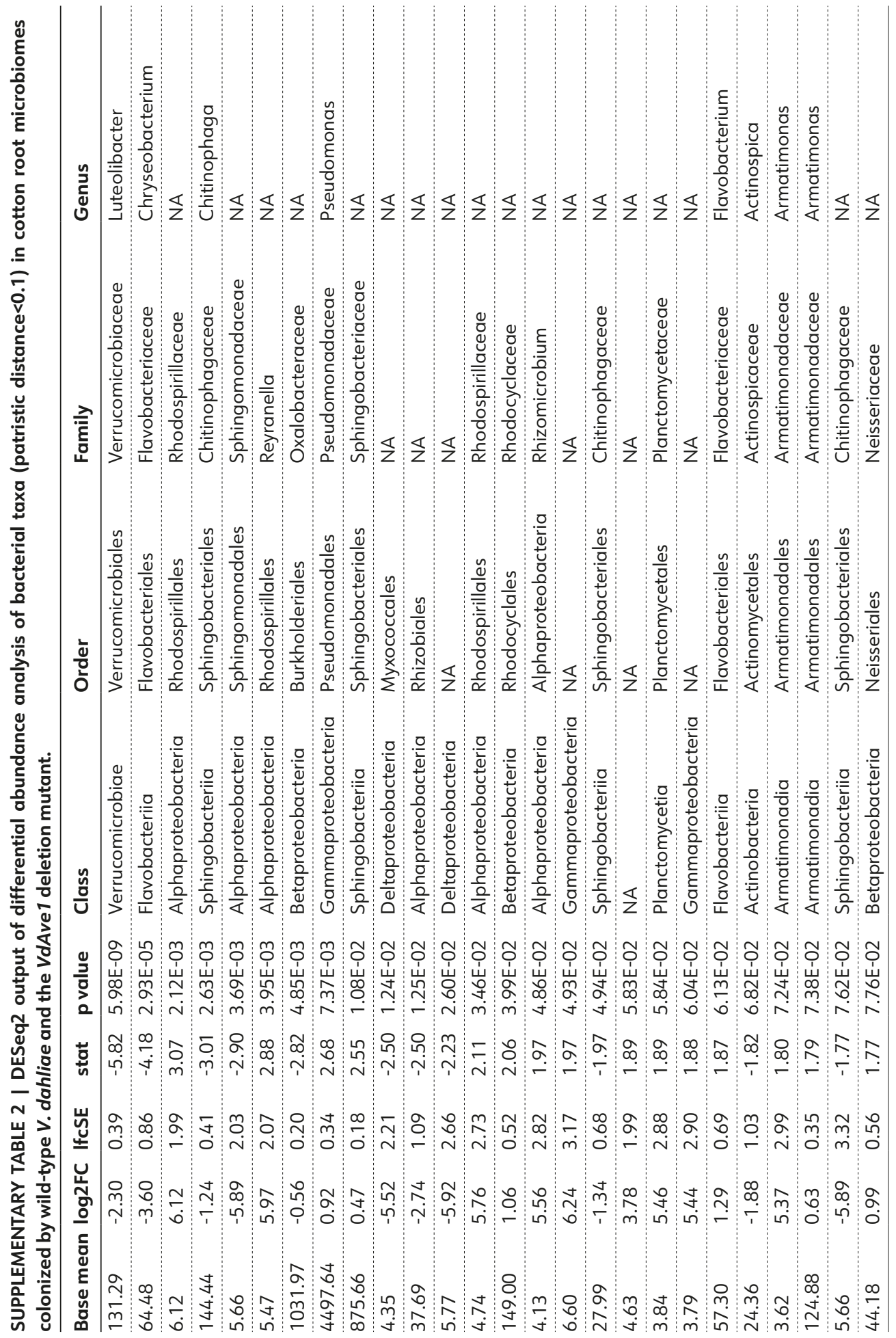




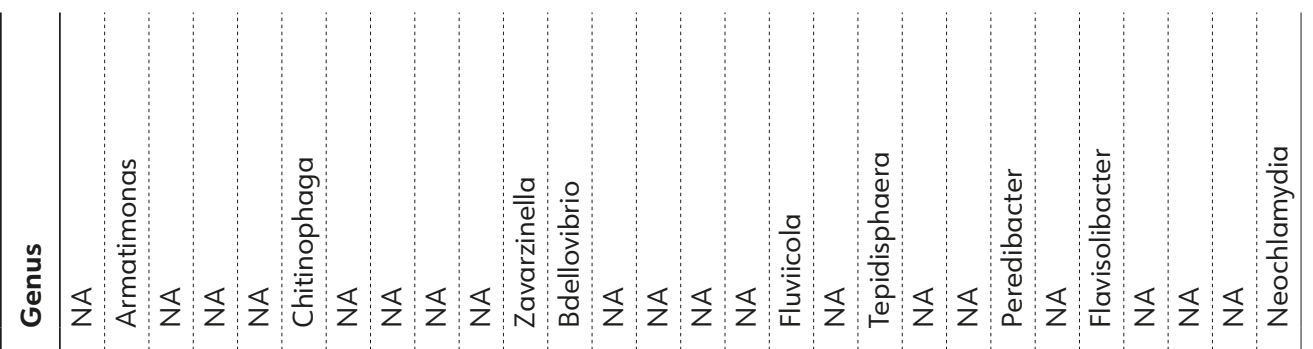

产

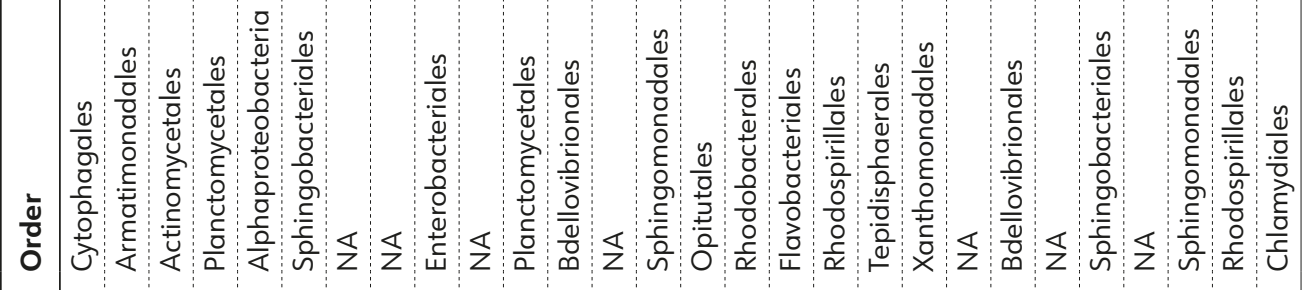

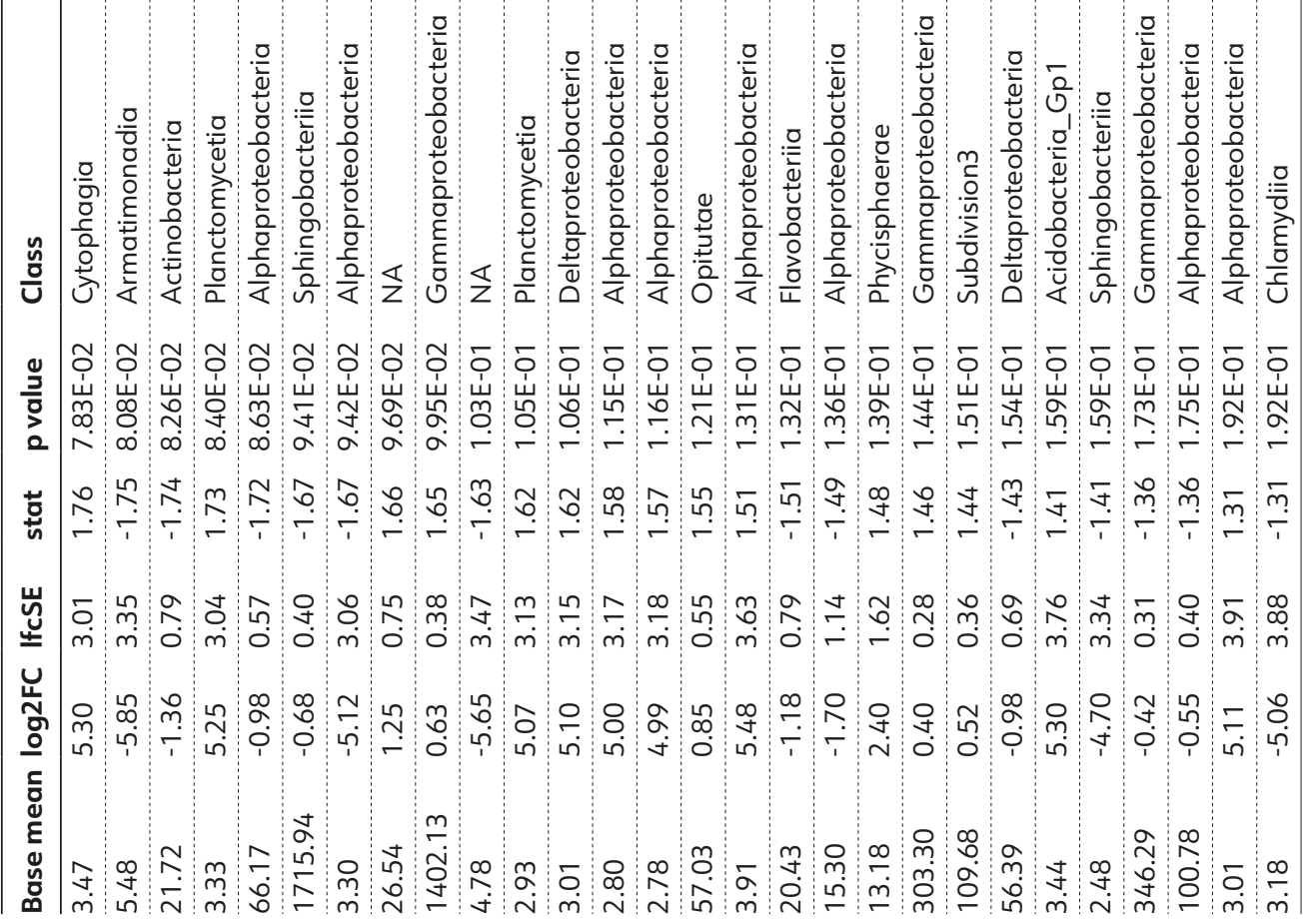




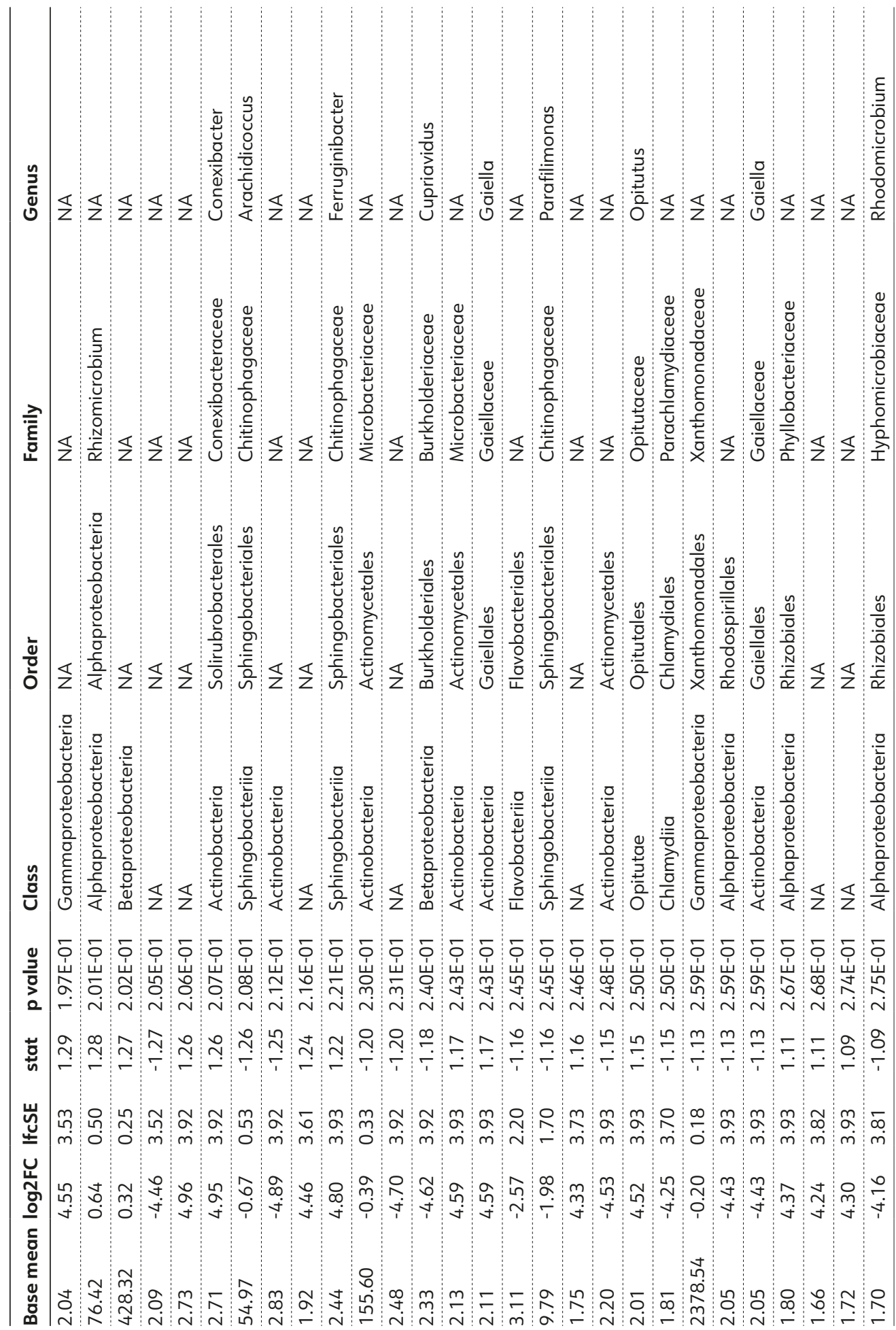




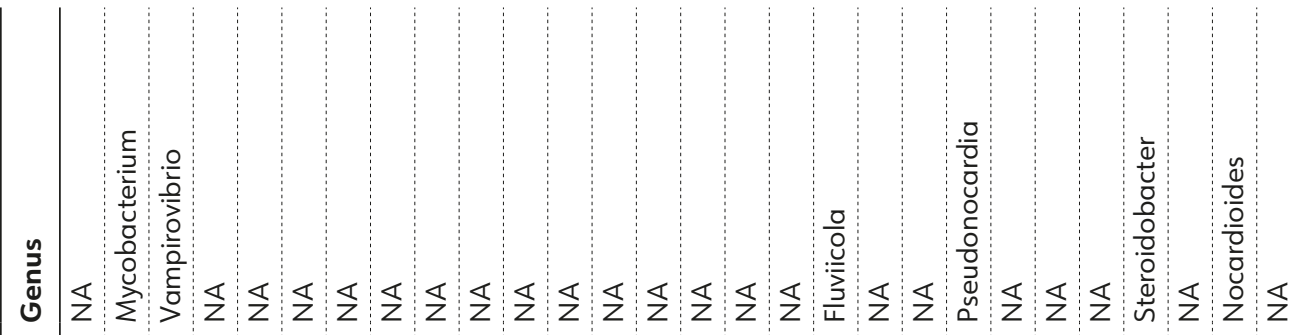

\

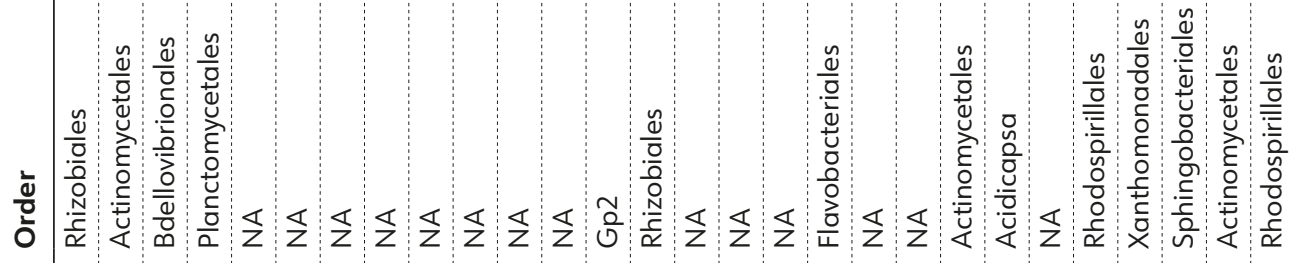

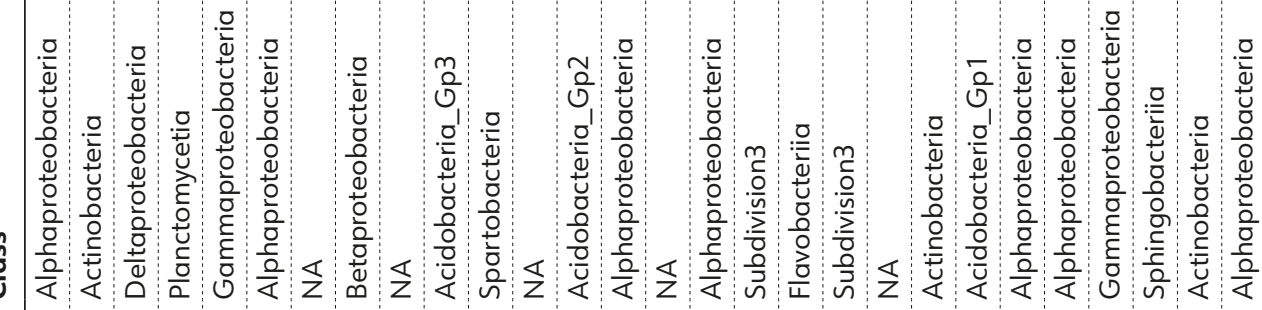

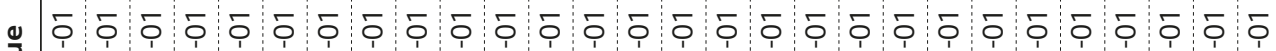
J

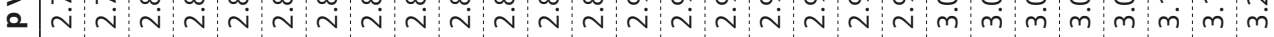
च゙

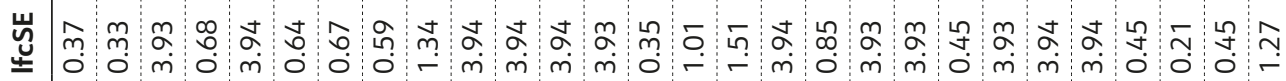
ư ゙ָ 항

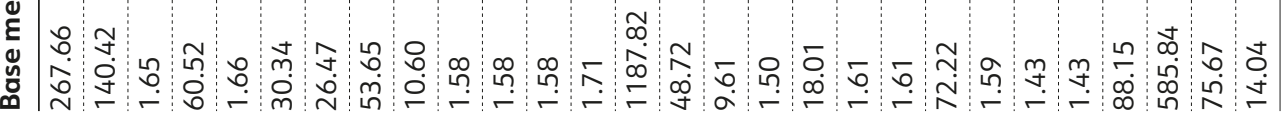



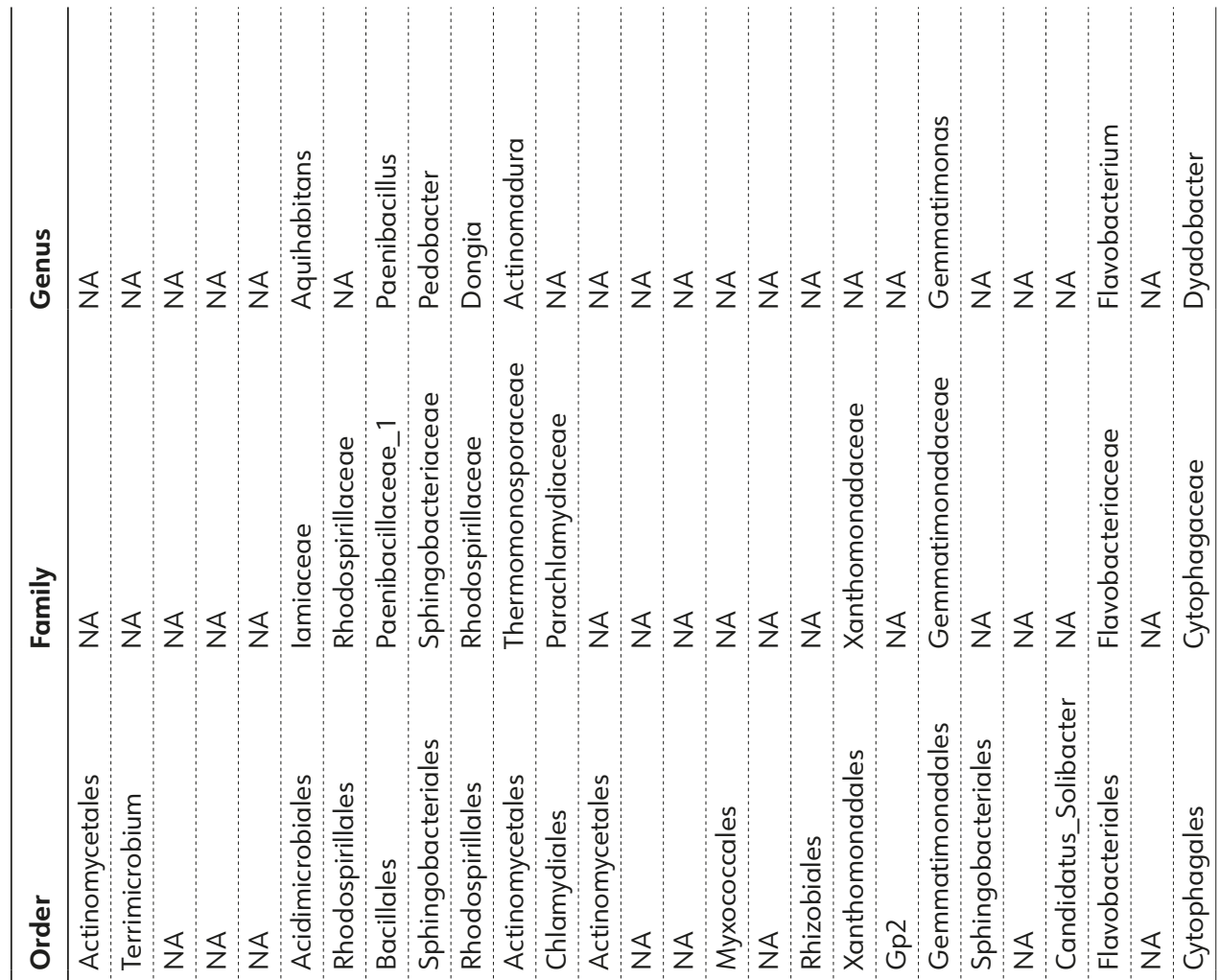

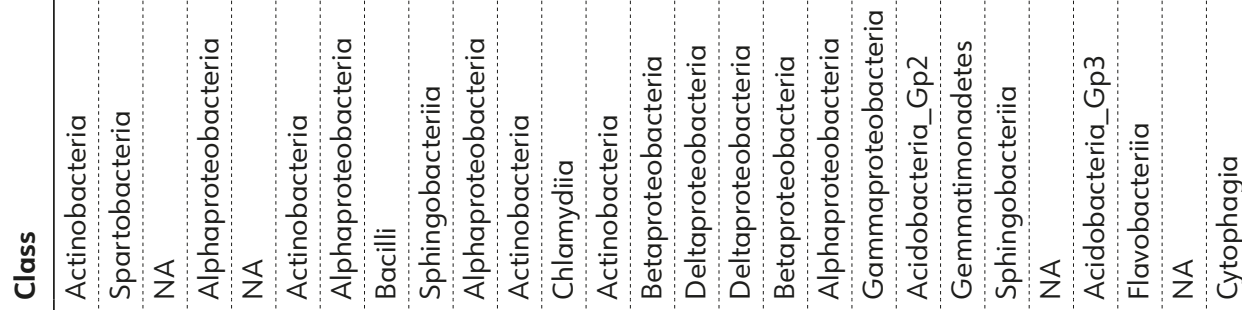
\ J

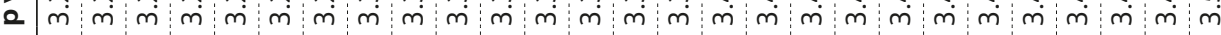

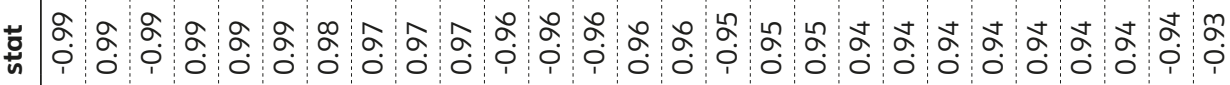

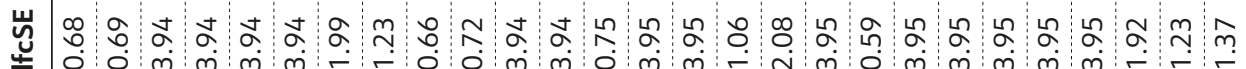
茨 o है

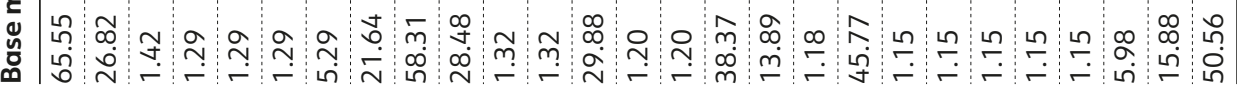




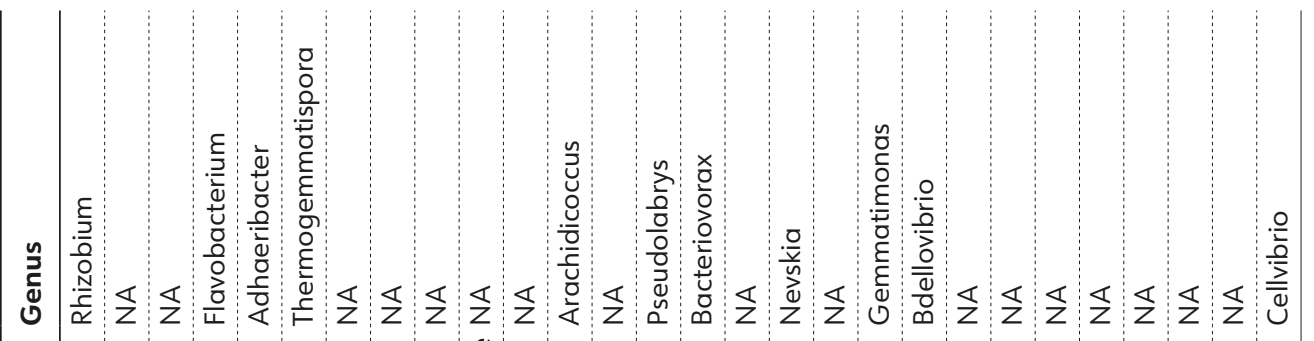

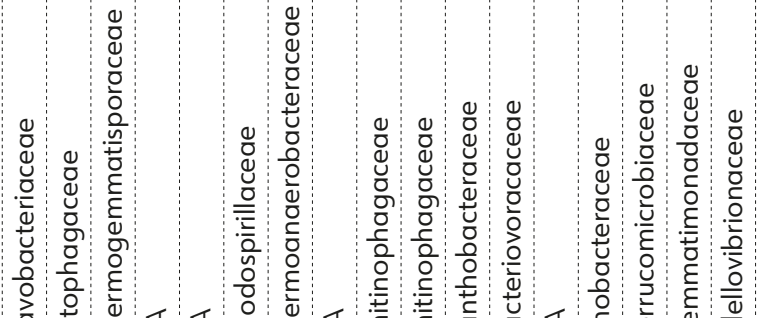

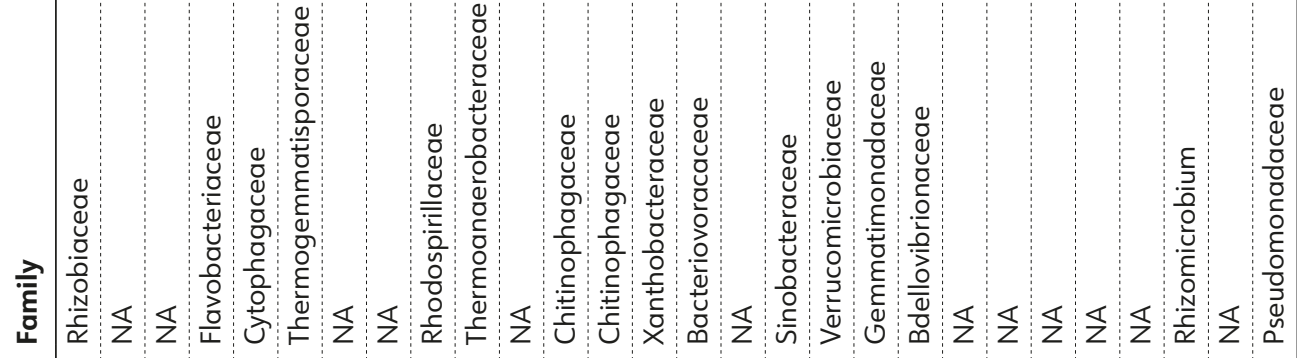

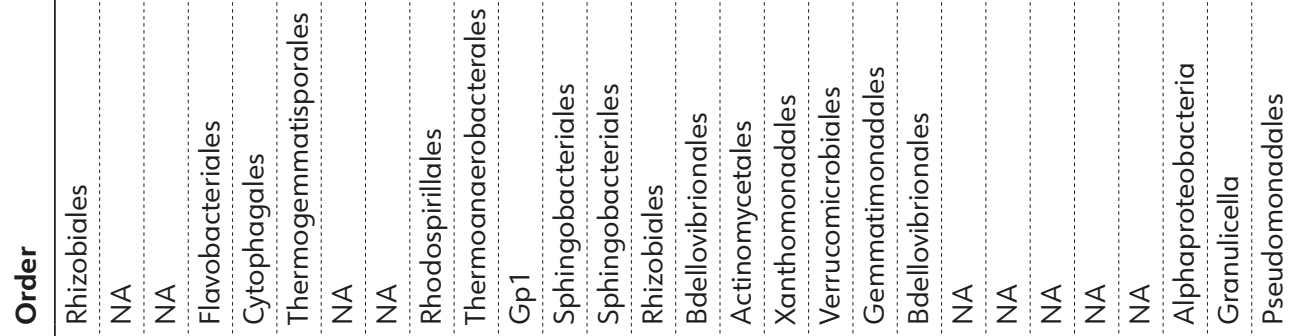

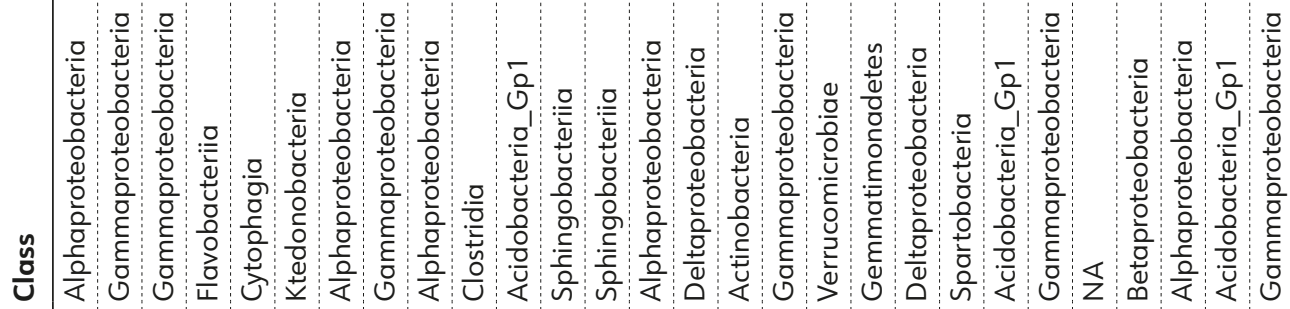

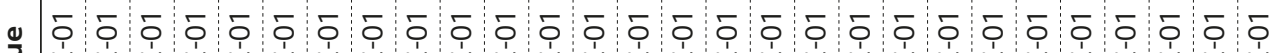
כ흐

$a \dot{m} \dot{m} \dot{m} \dot{m} \dot{m} \dot{m} \dot{m} \dot{m} \dot{m} \dot{m} \dot{m} \dot{m} m \dot{m} \dot{m} \dot{m} \dot{m} \dot{m} \dot{m} \dot{j}$

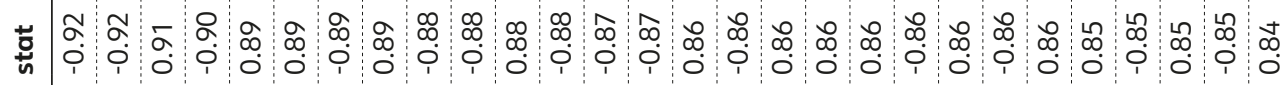

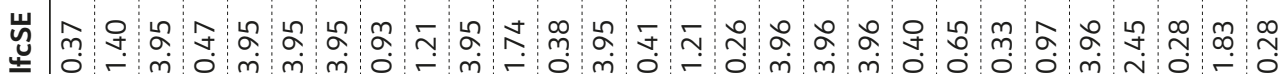

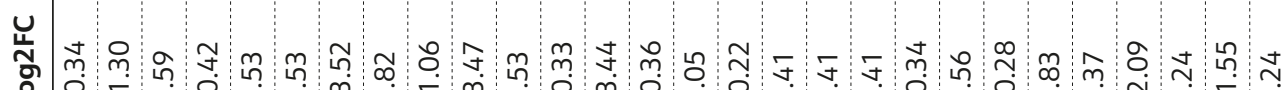
을 잉

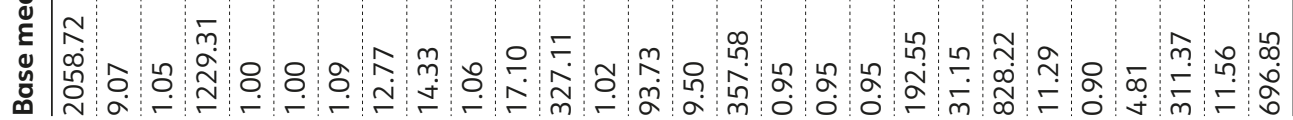




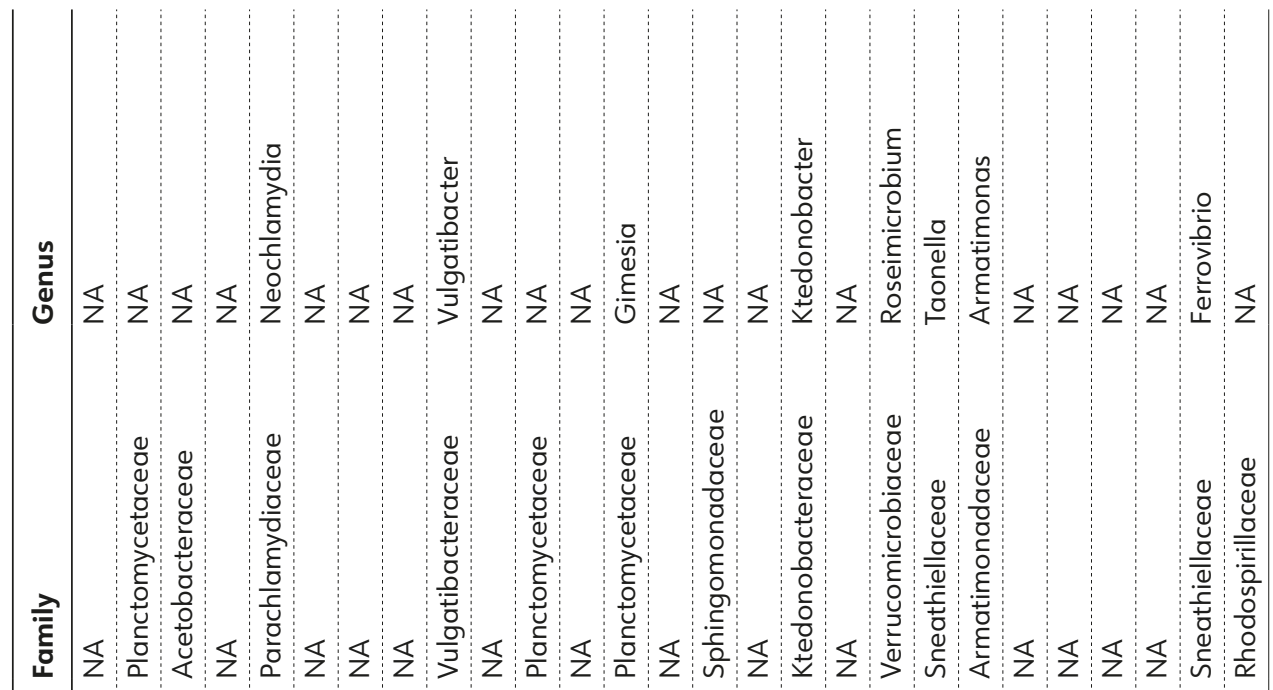

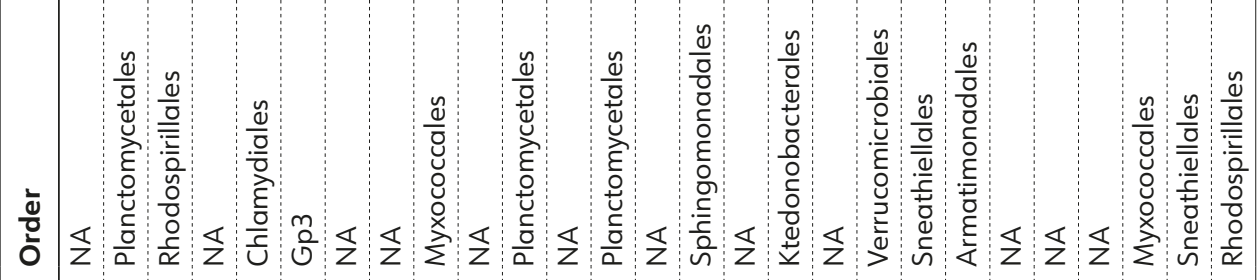

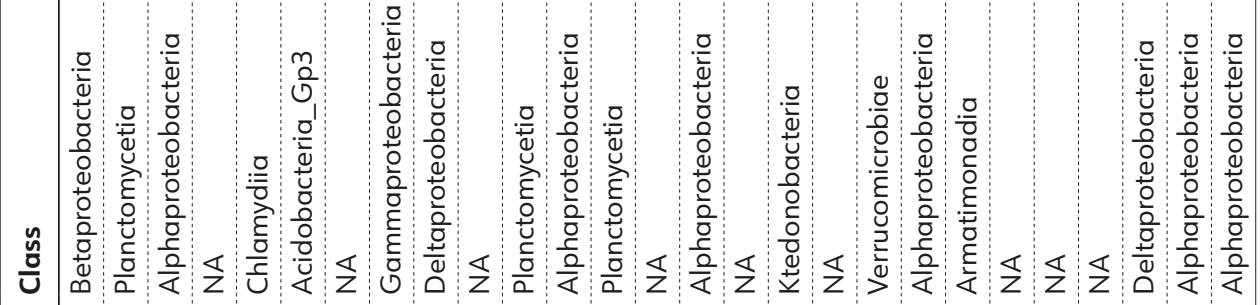

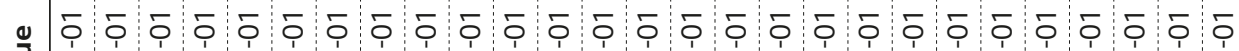
J

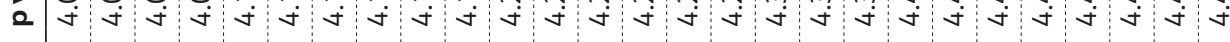

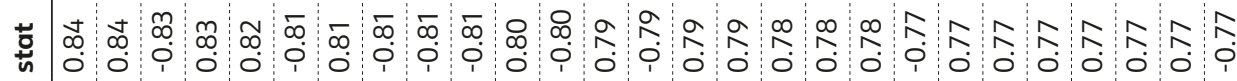
岂 u

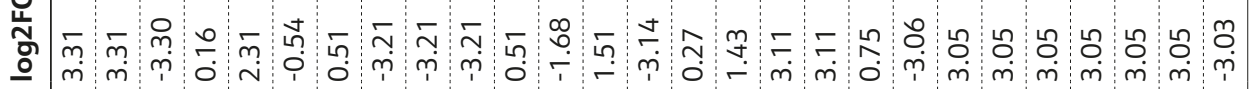
है

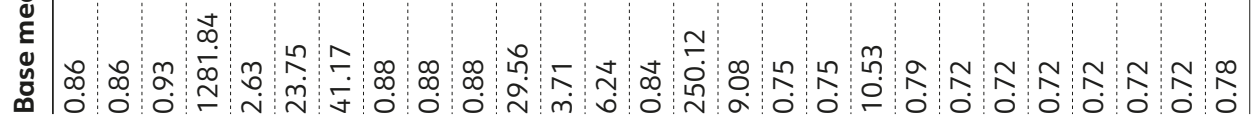




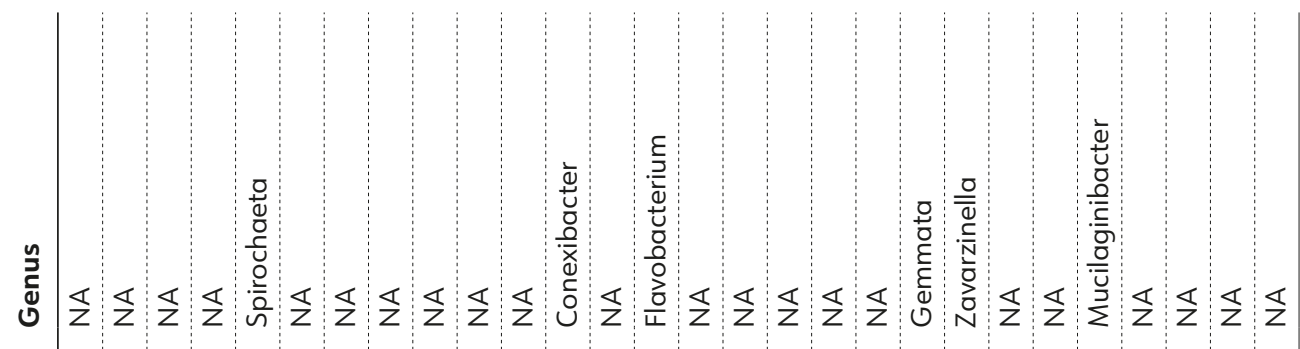

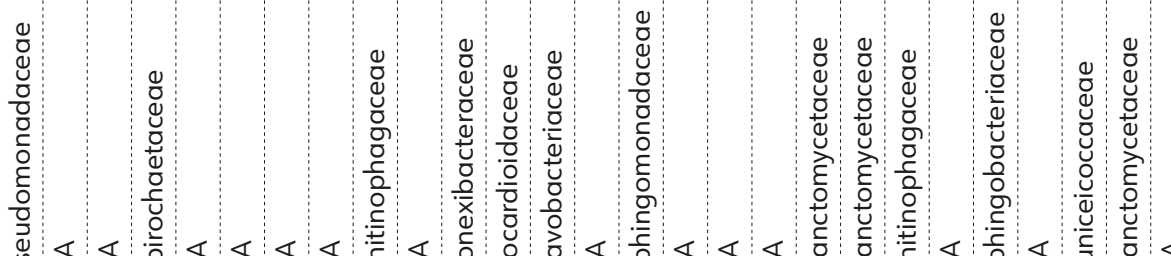

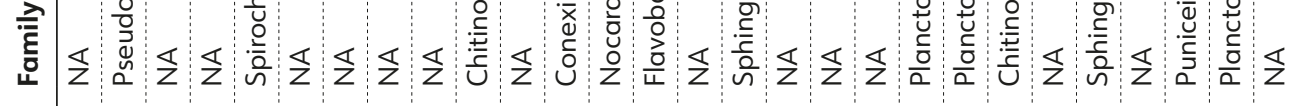

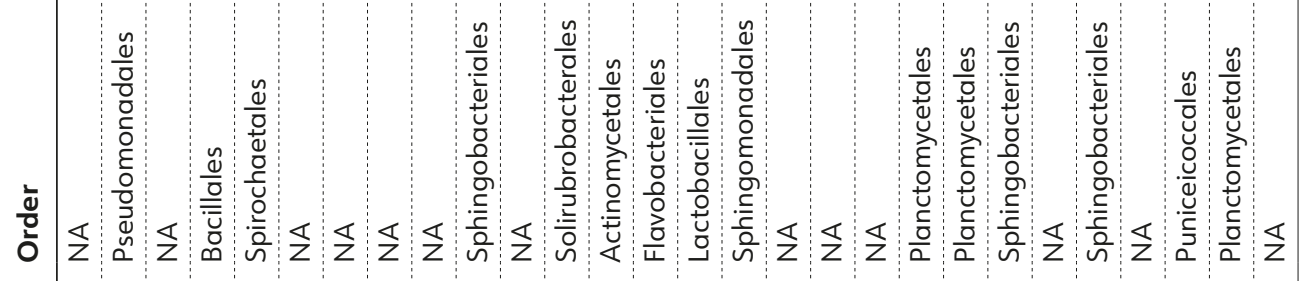

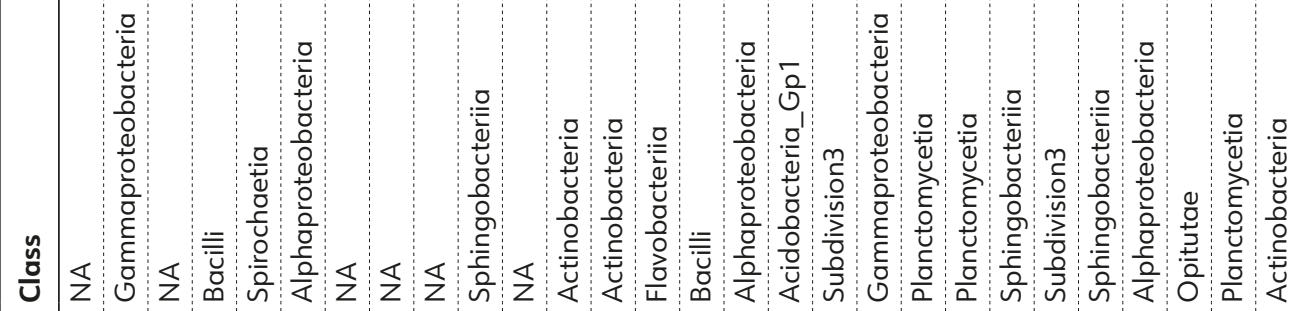

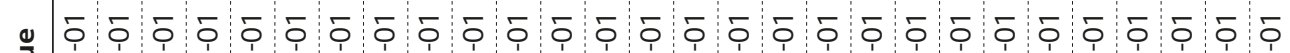

J

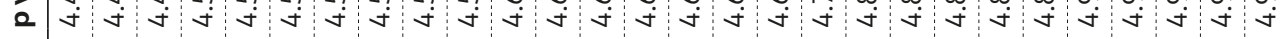

岂

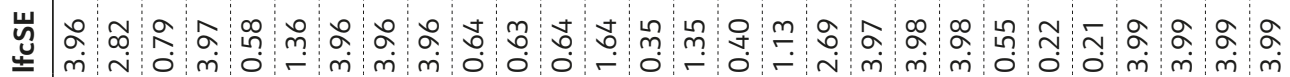

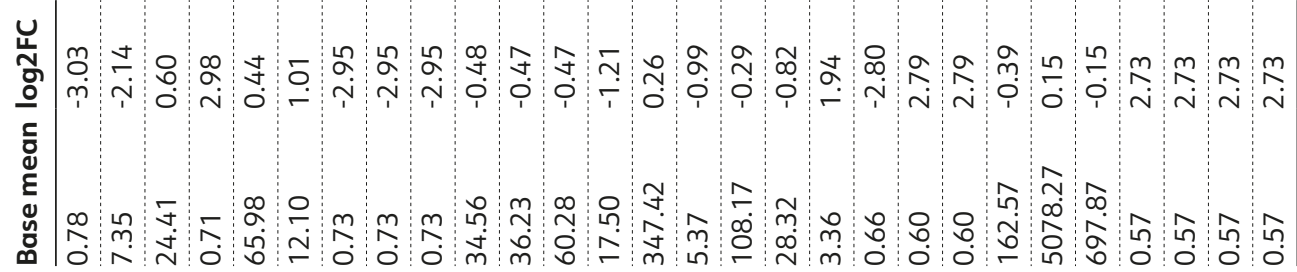




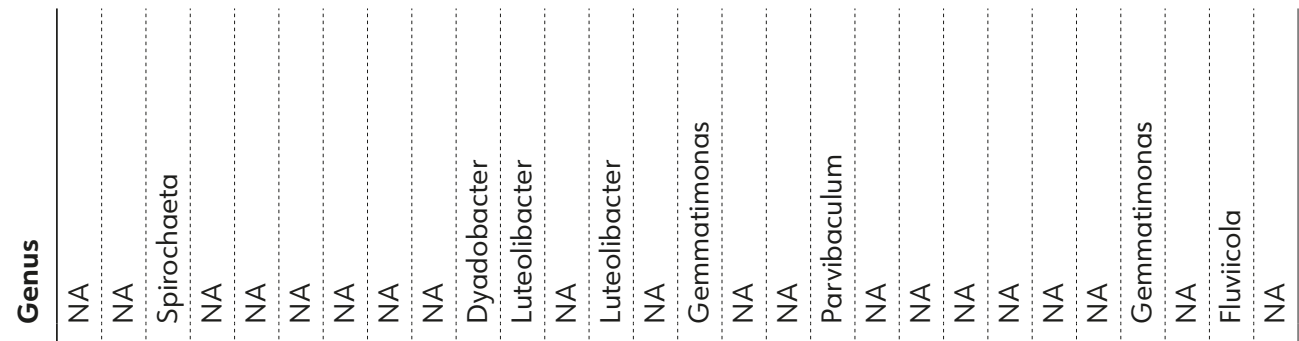

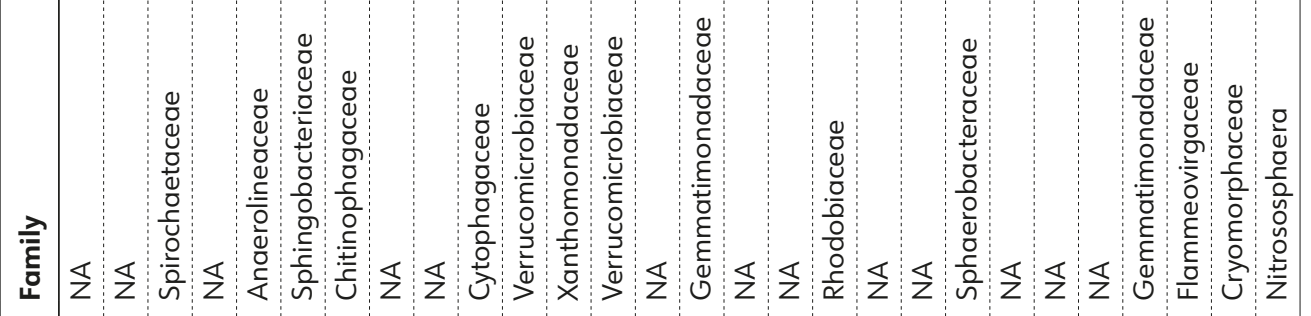

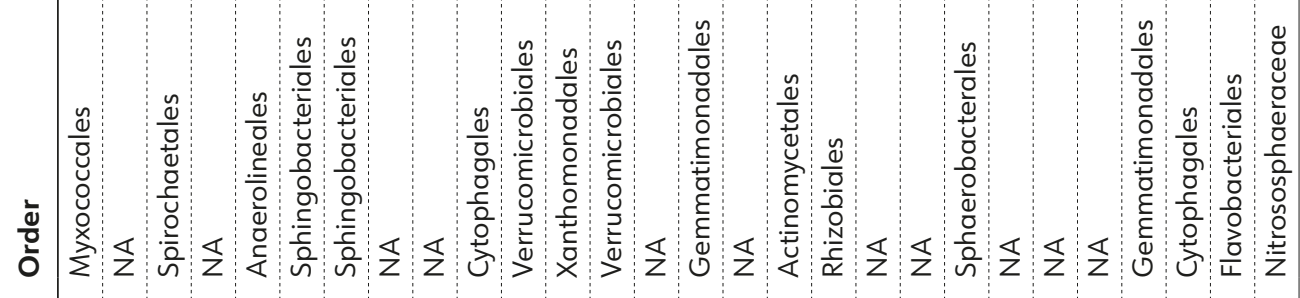

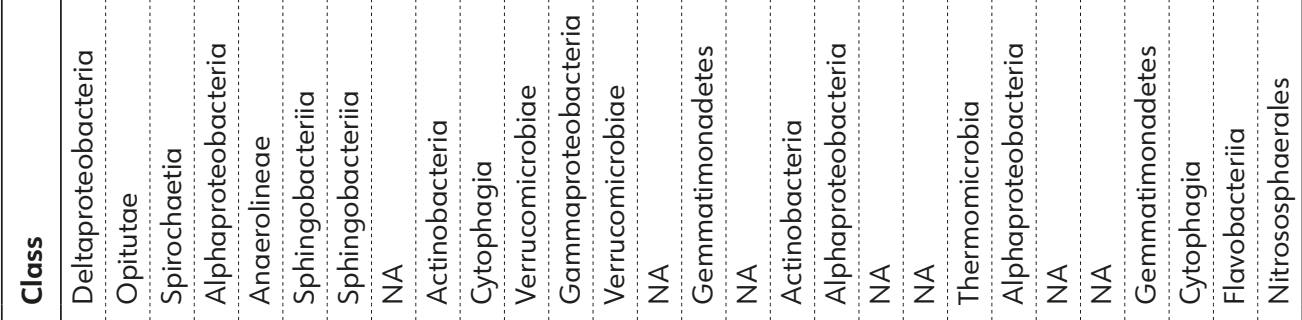

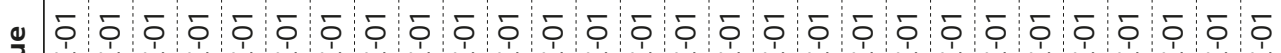
J

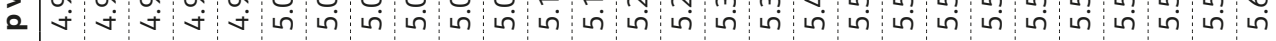

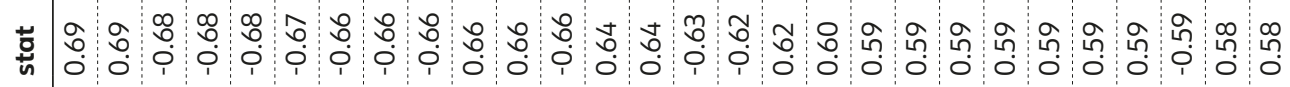

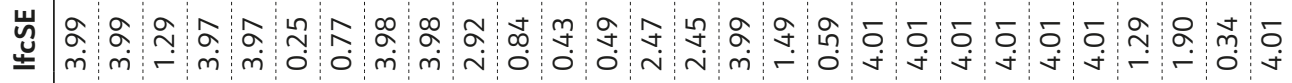

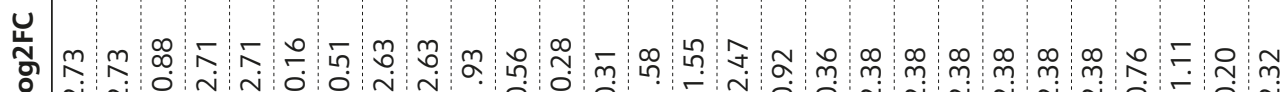
o

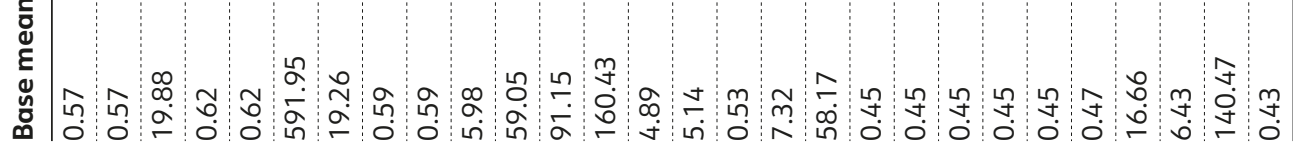




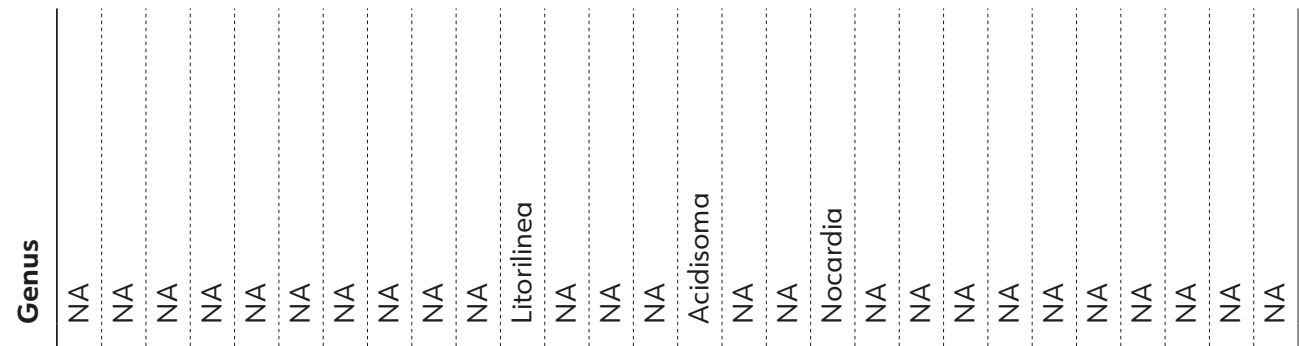

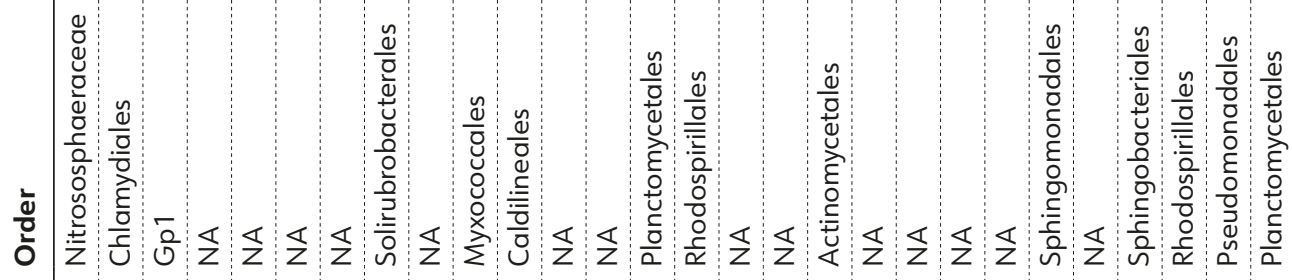

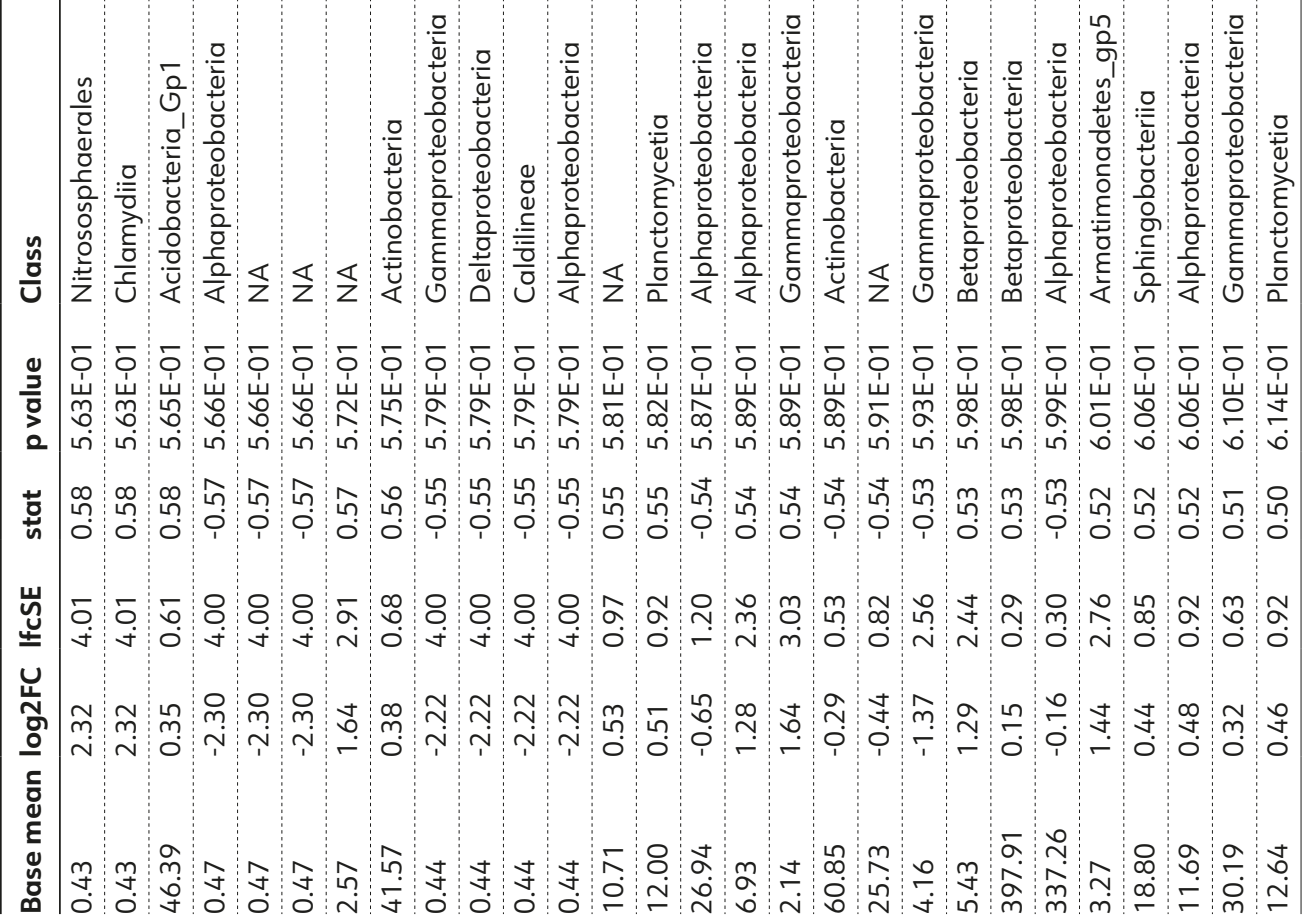




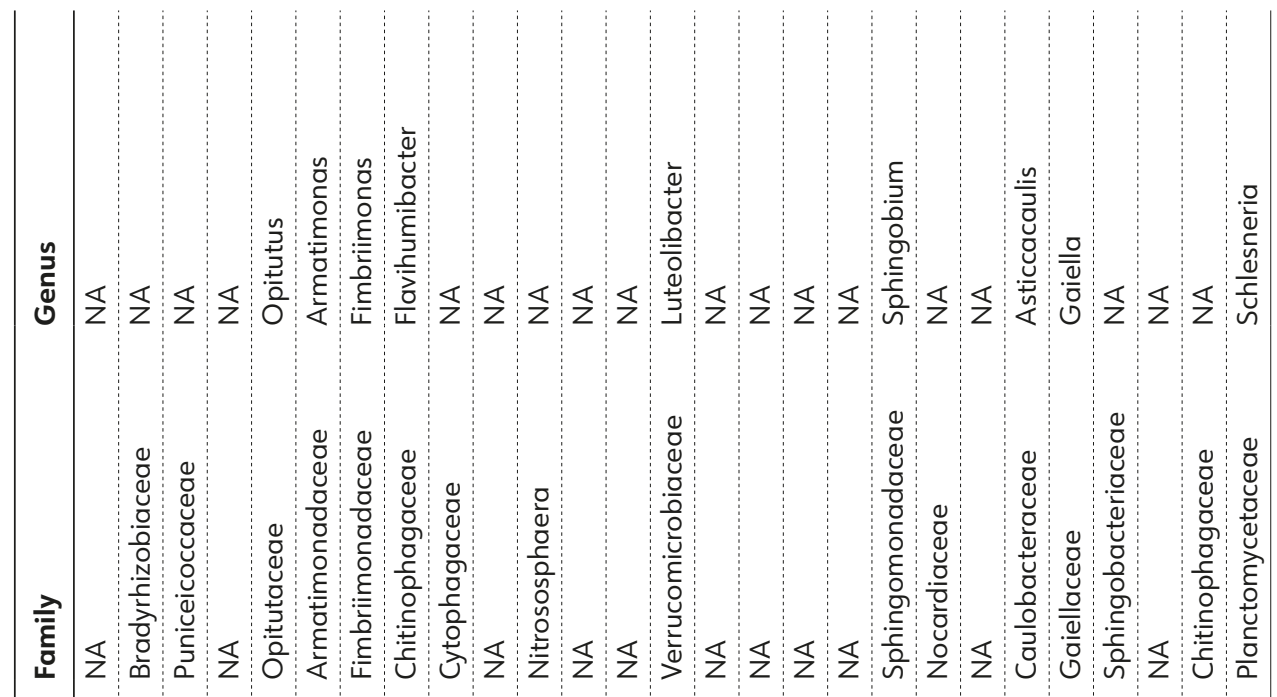

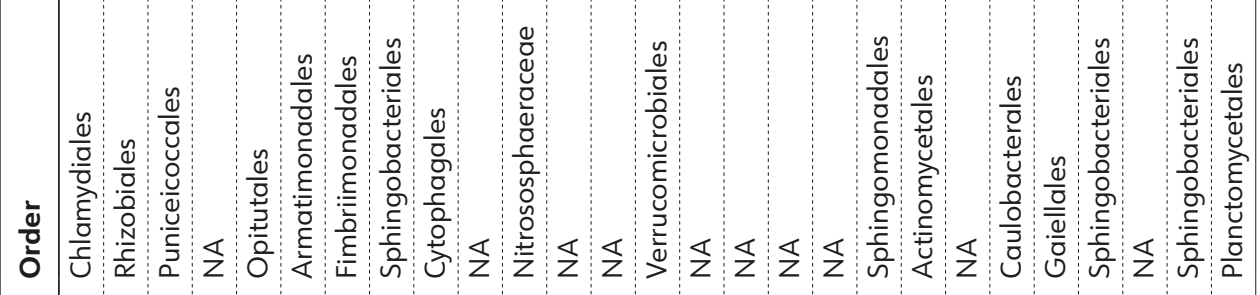

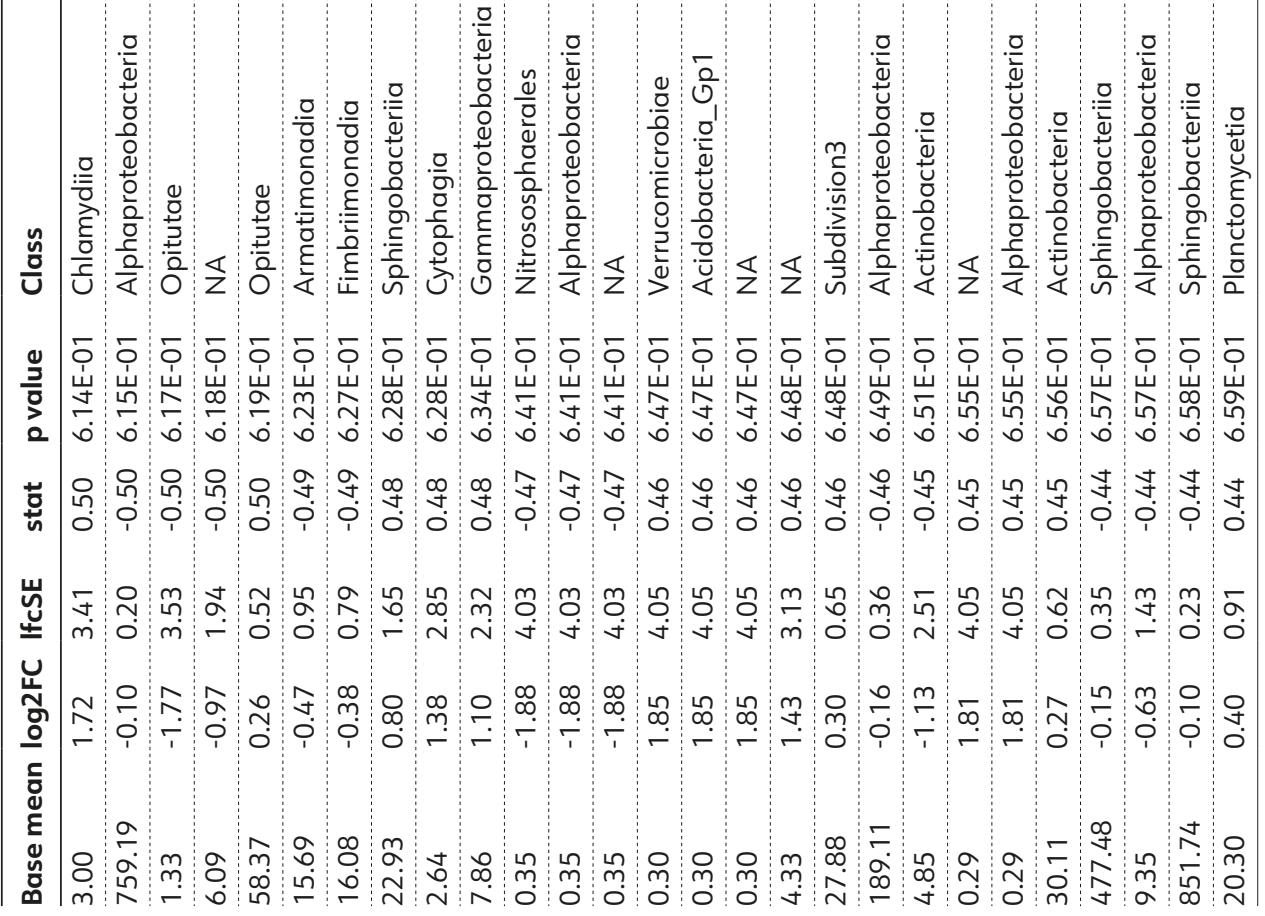




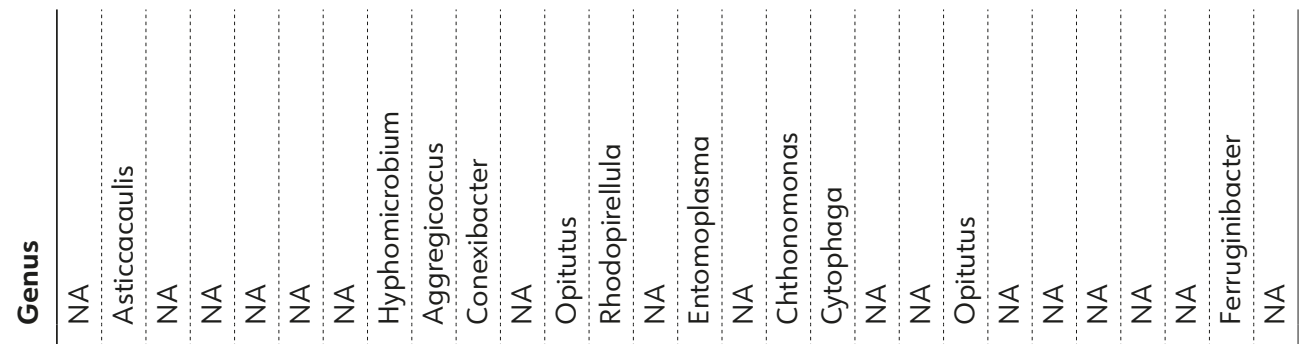

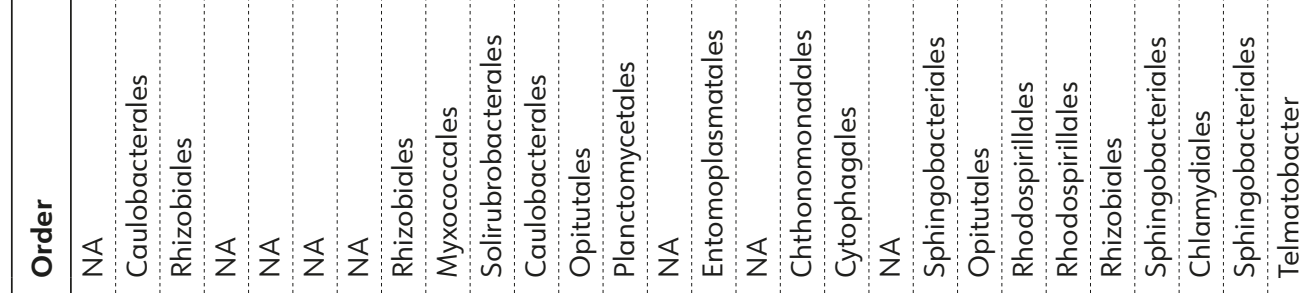

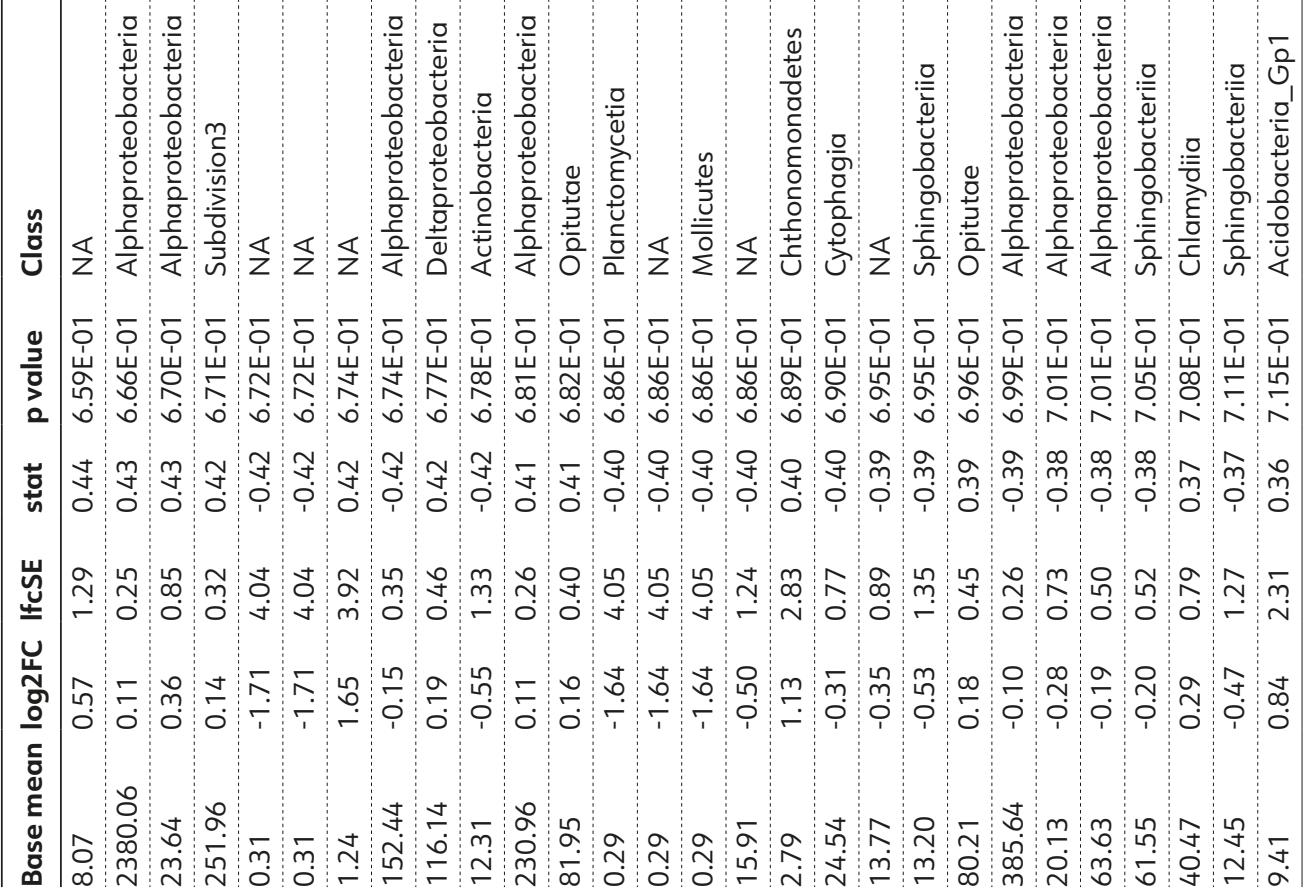




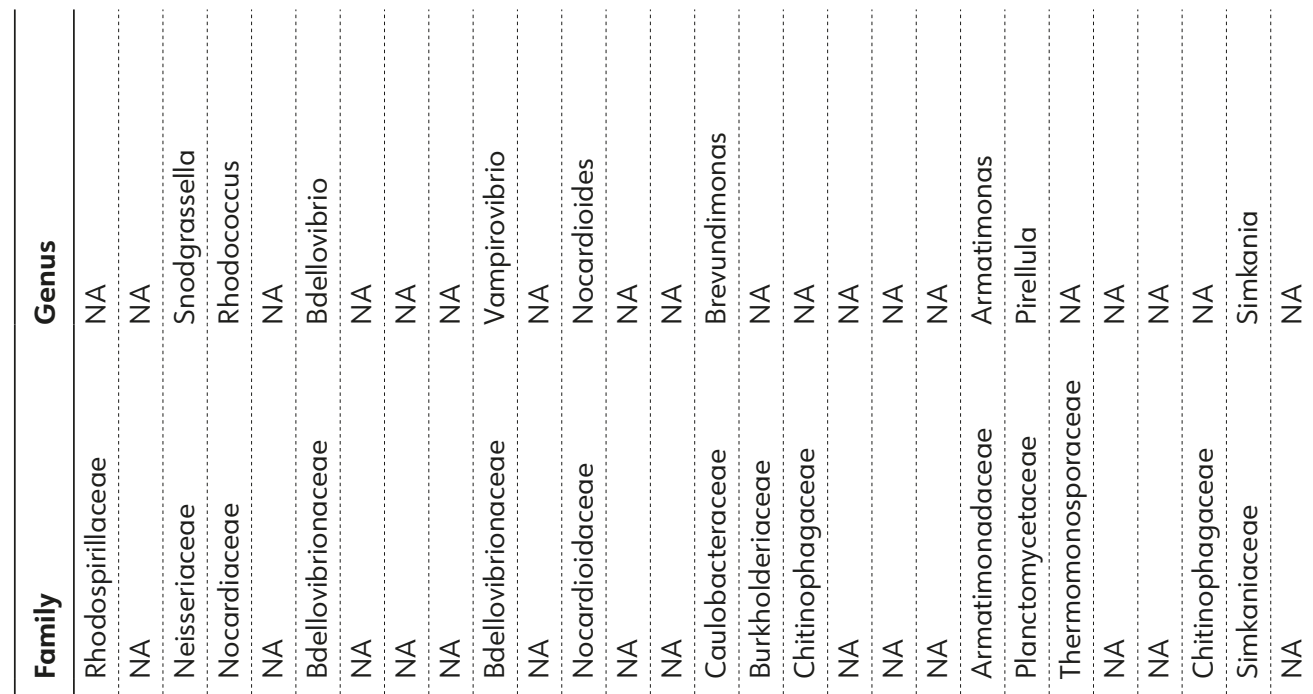

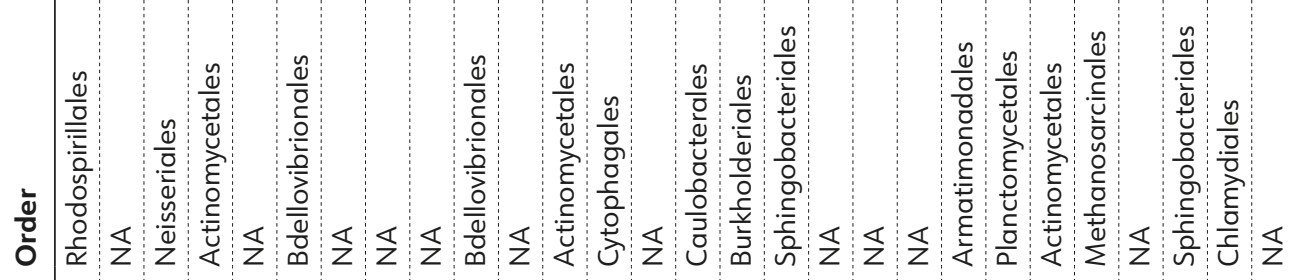

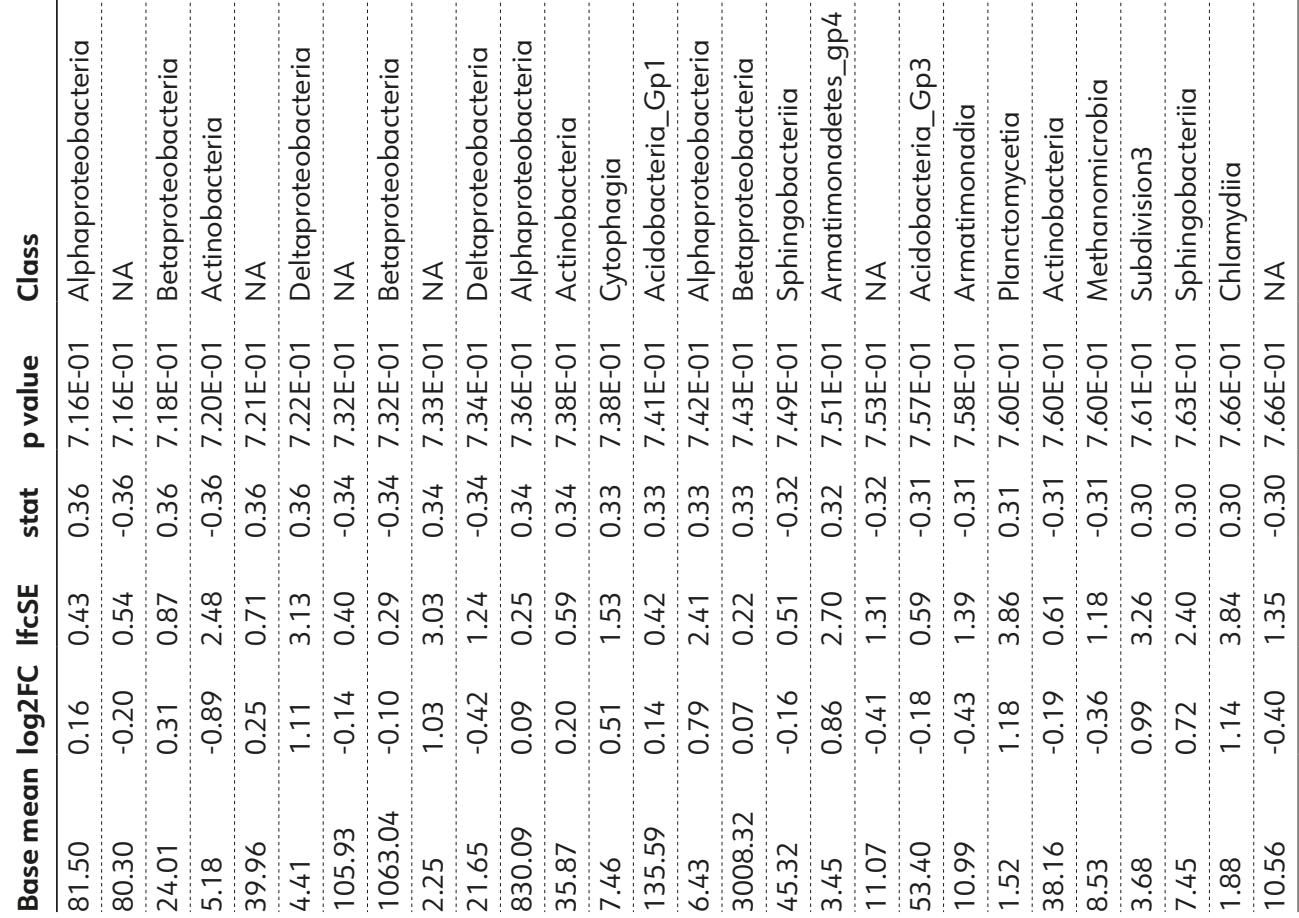




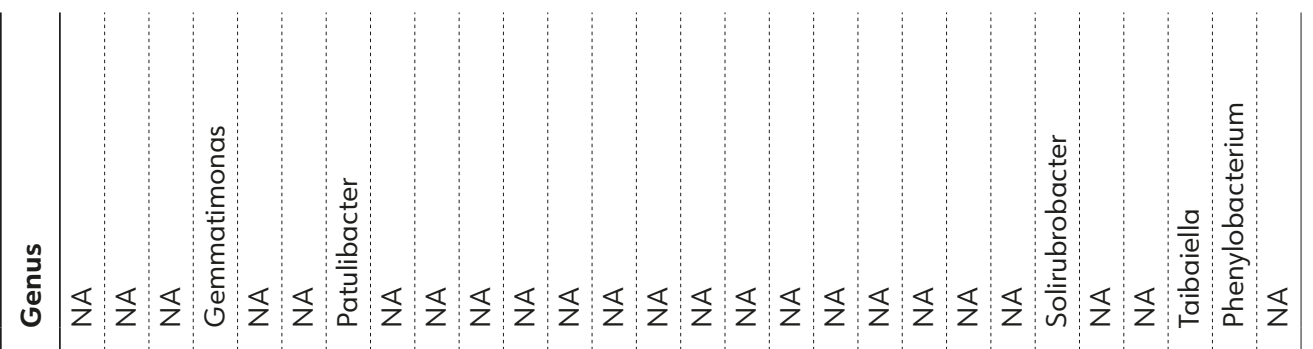
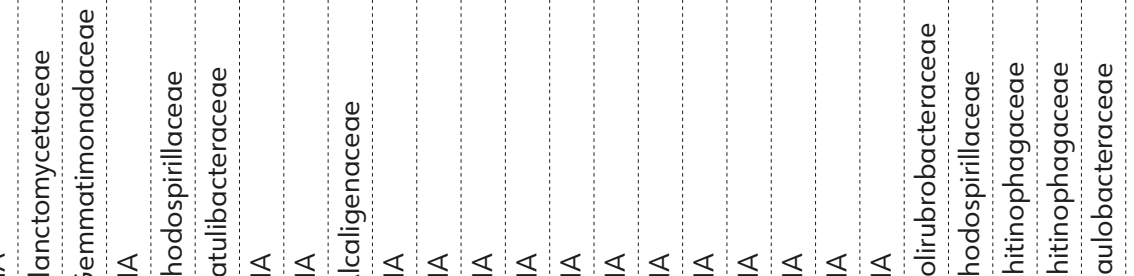

童

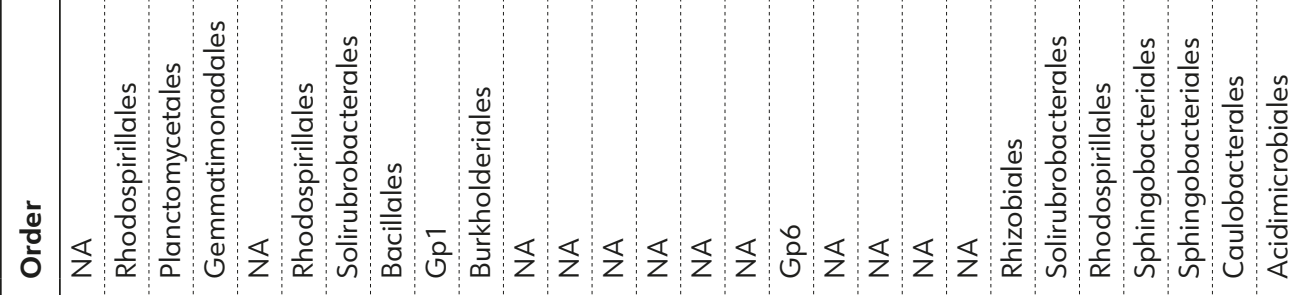

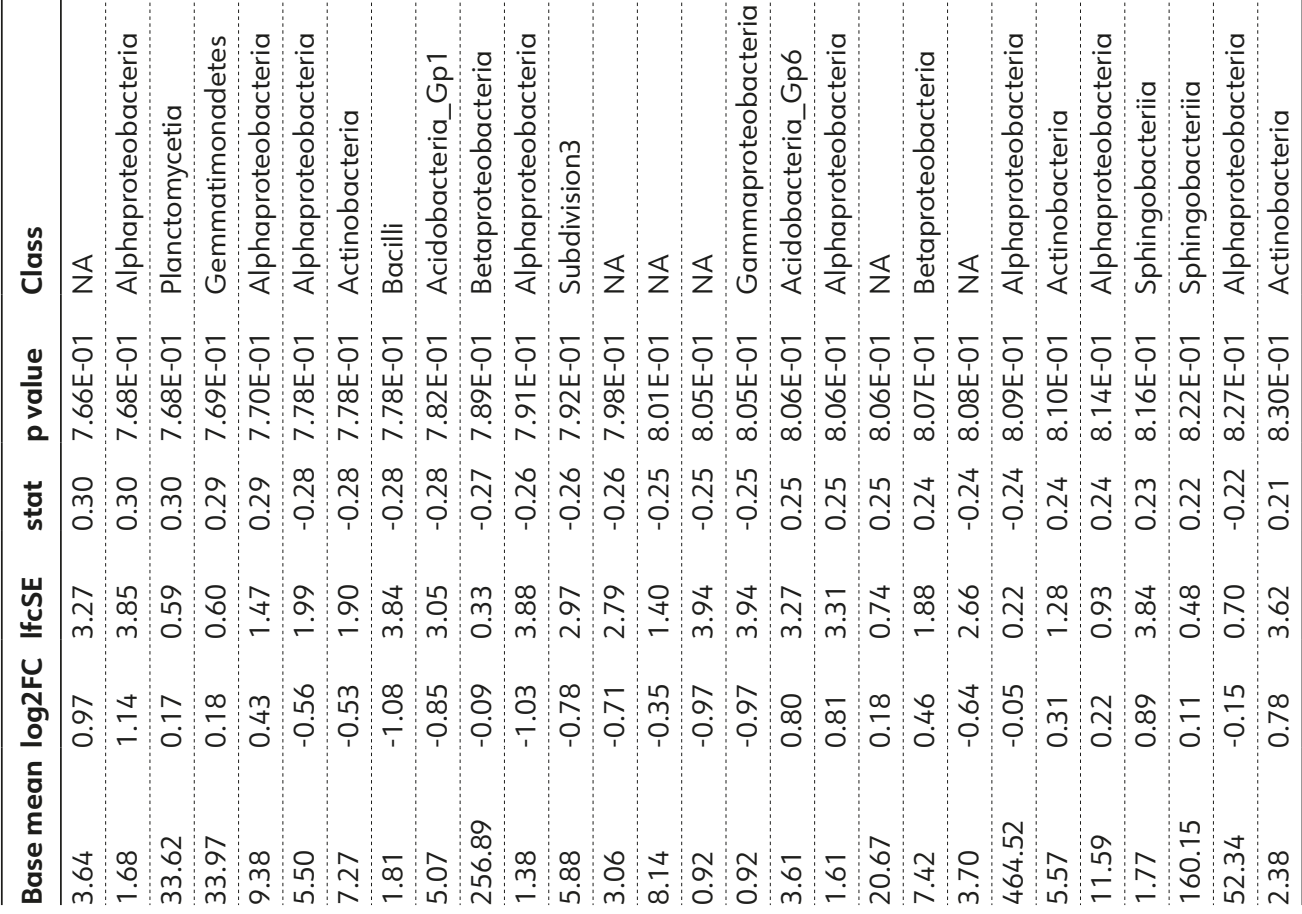




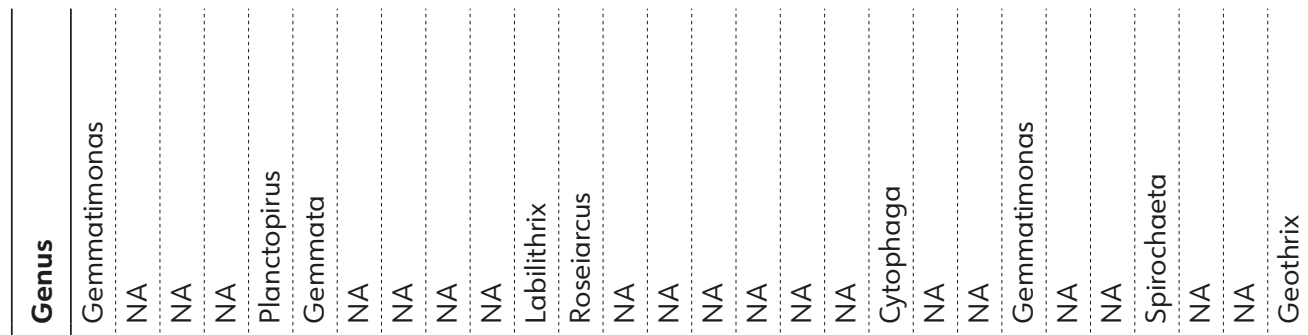

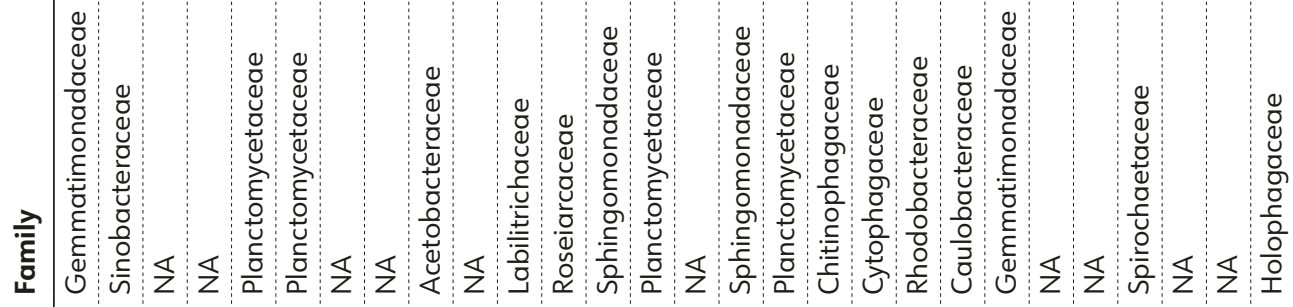

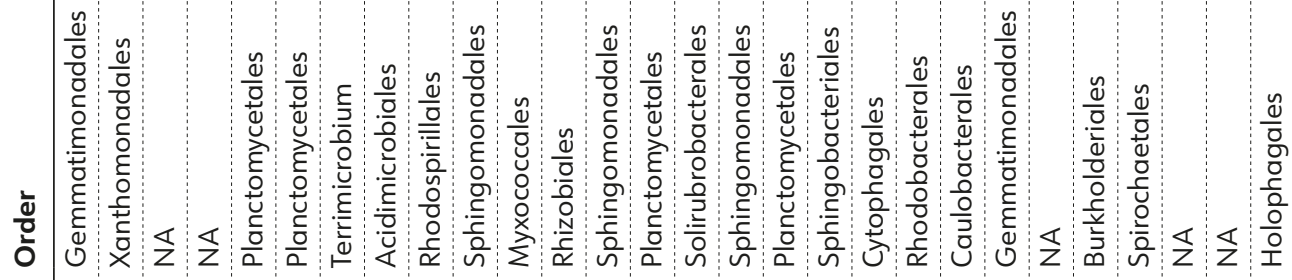

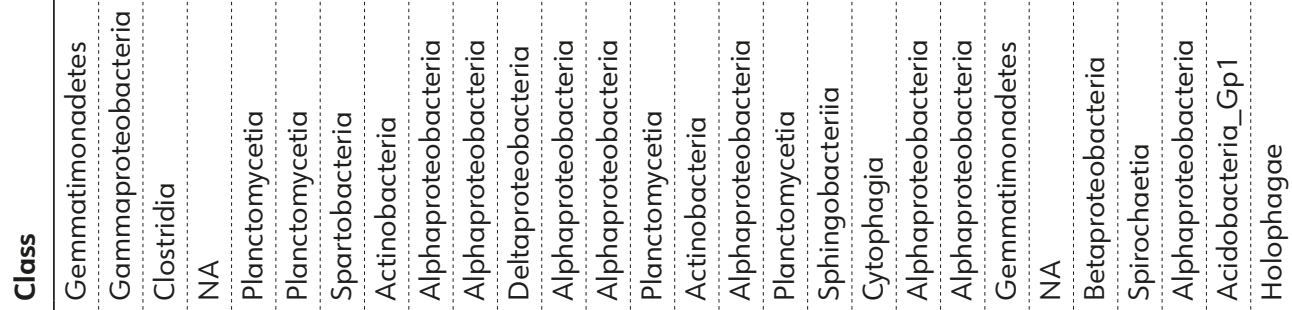
ఎ б б б б б б б б б б б б б б б б б б б б б б б כ

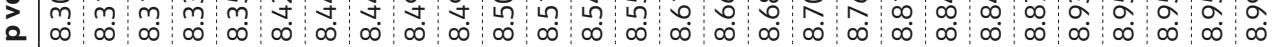

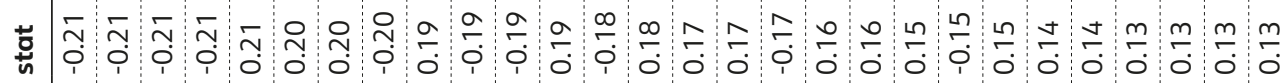
岂

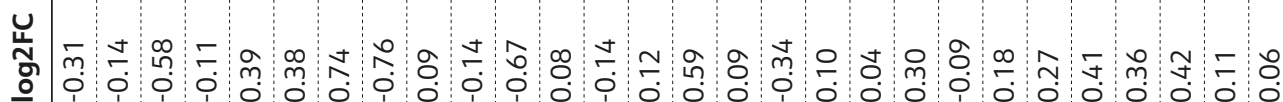

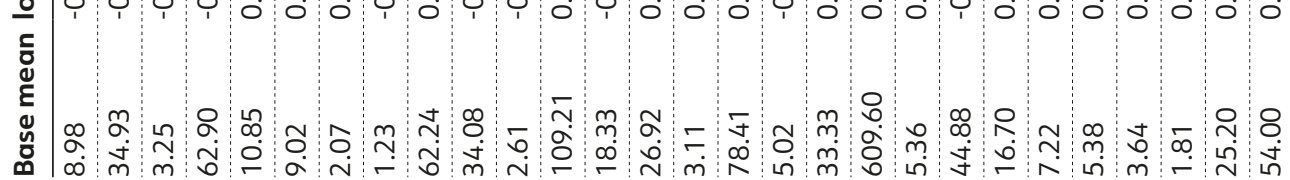




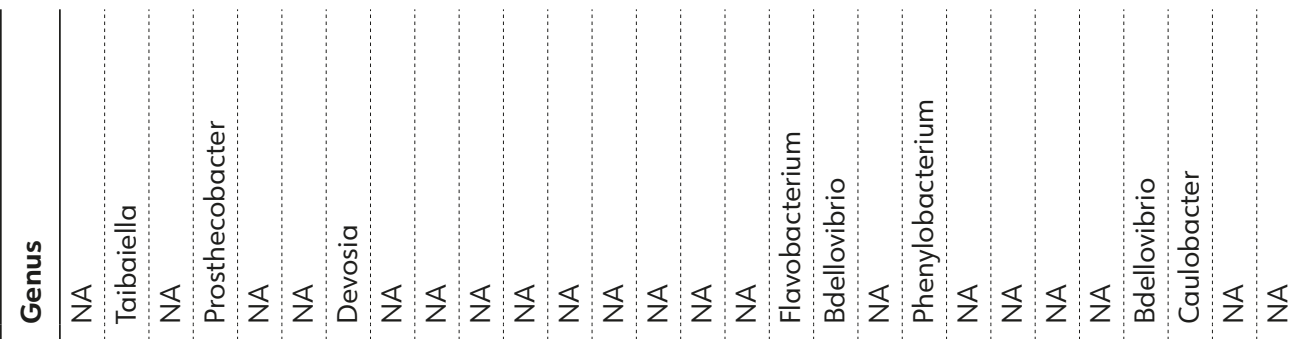

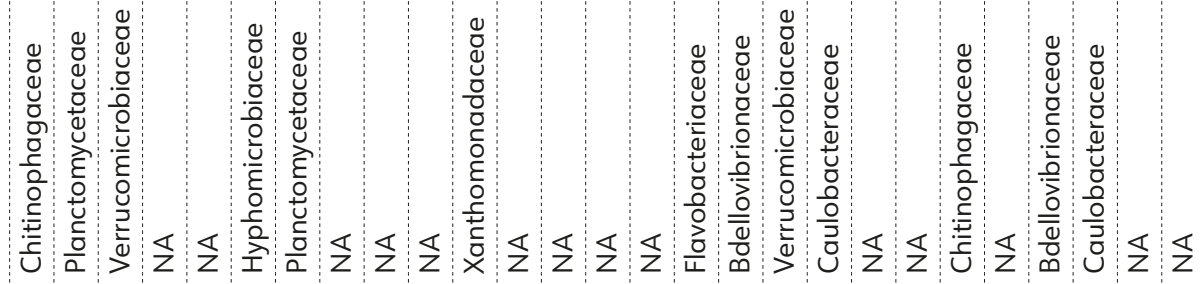

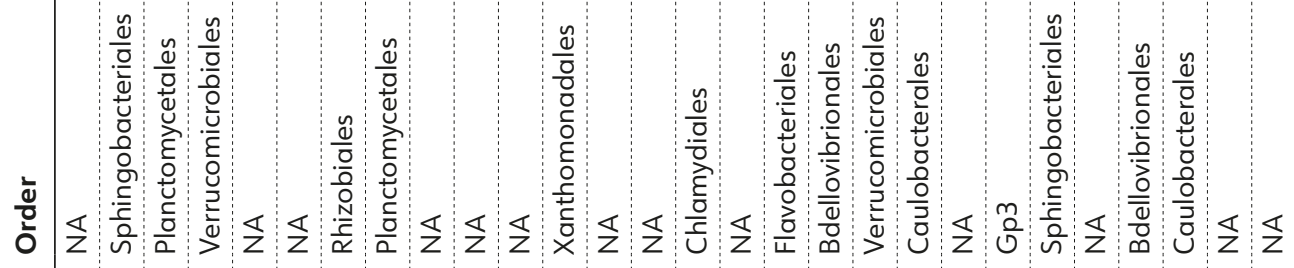

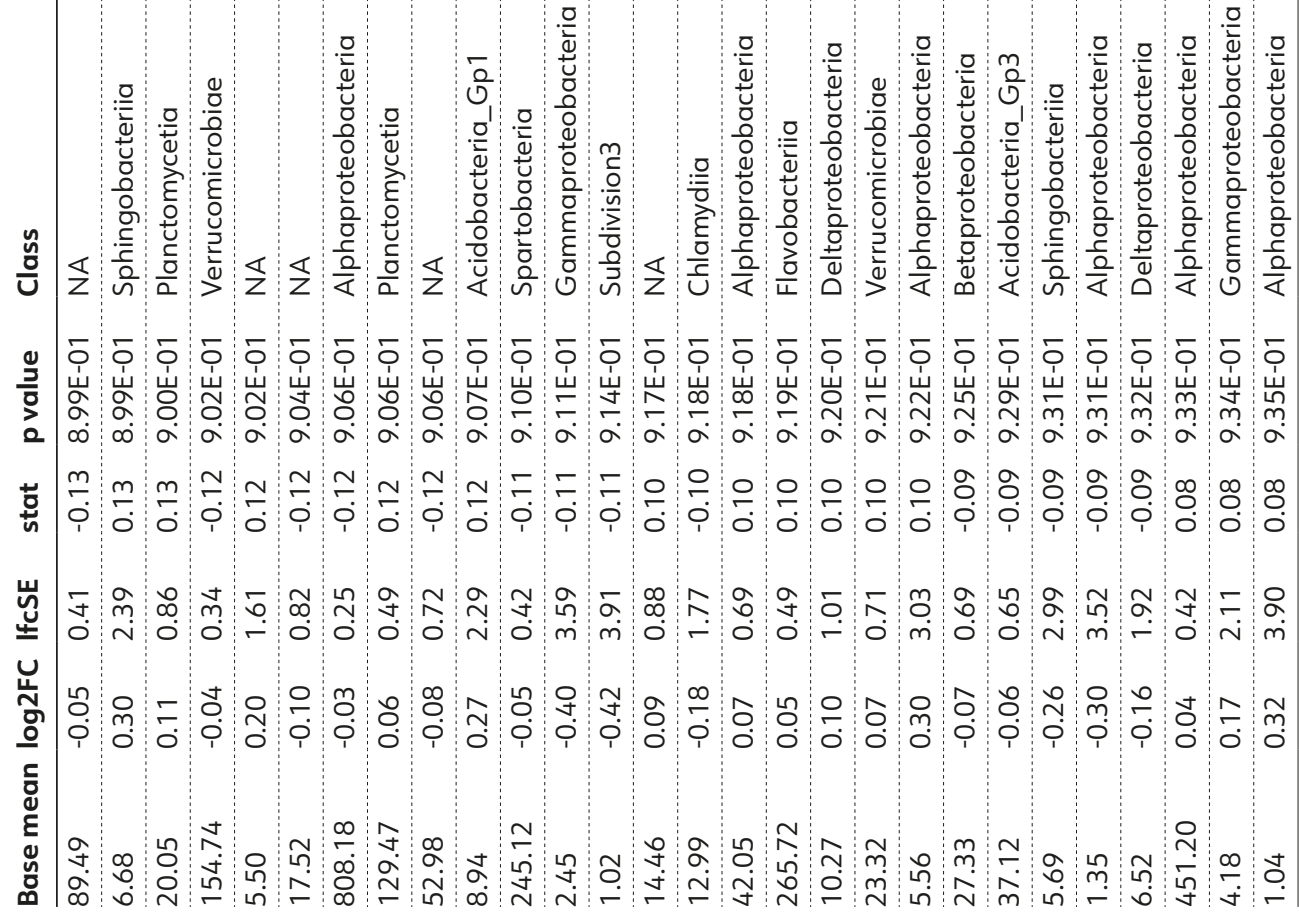




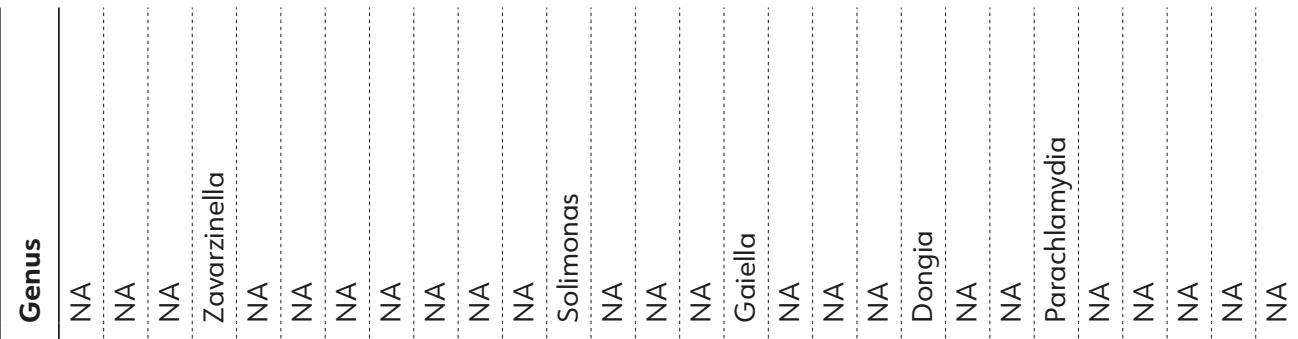

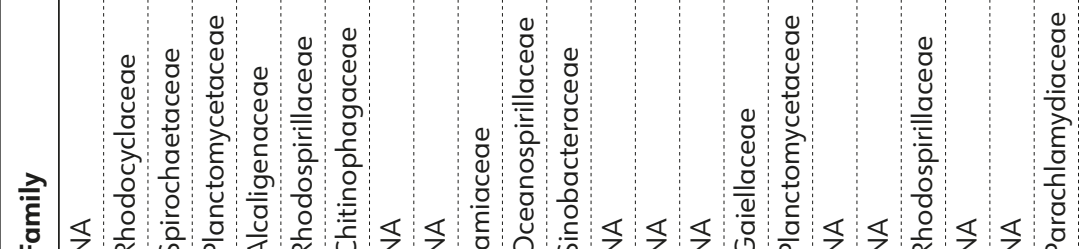

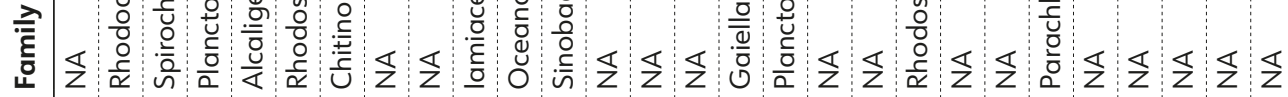

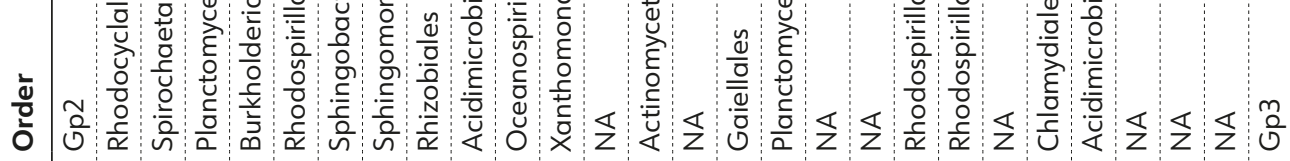

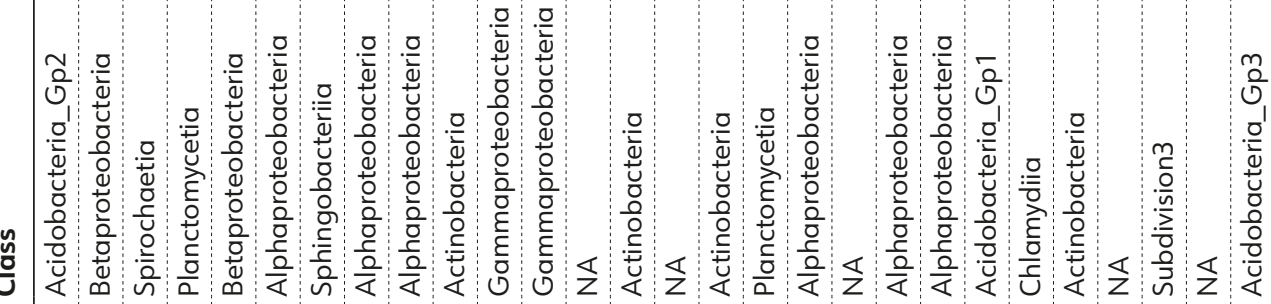

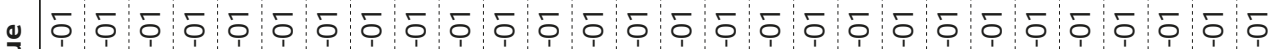
J

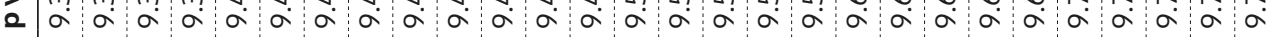

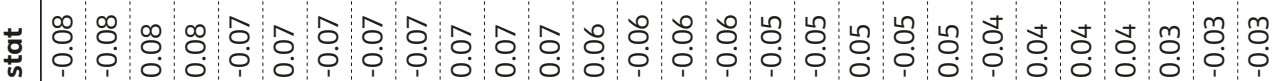

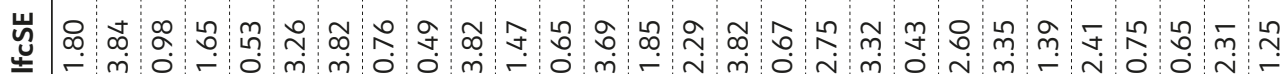

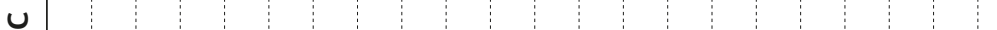

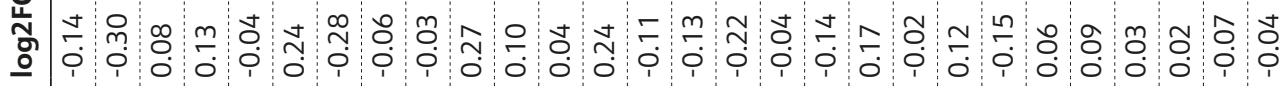
है

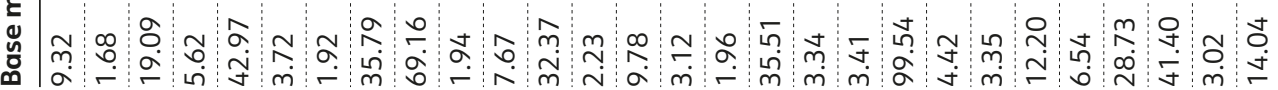



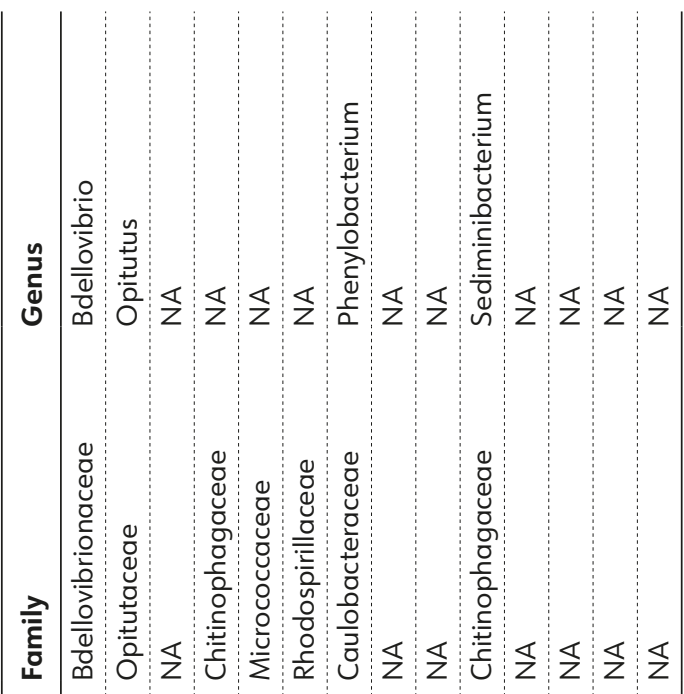

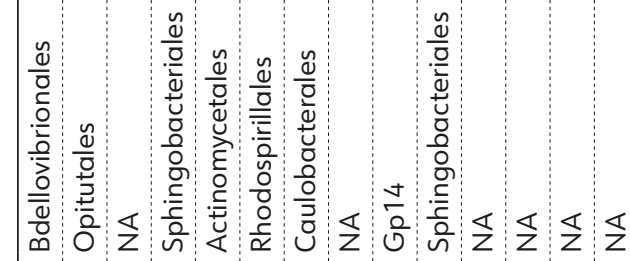

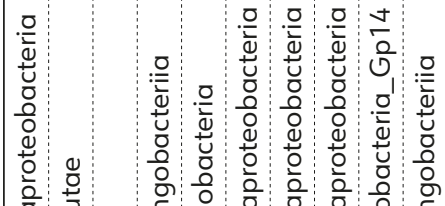

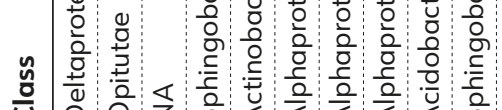

ป 는

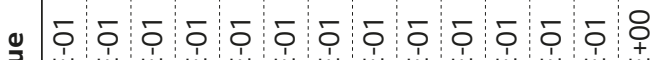

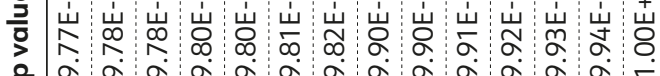

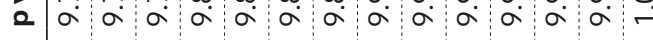

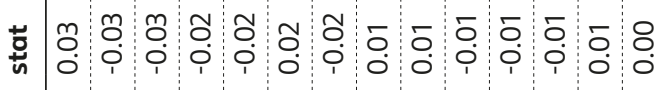

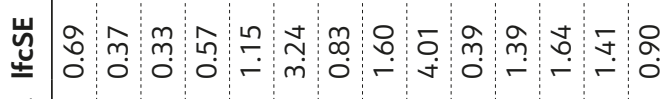

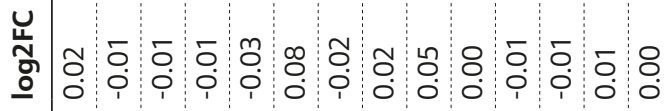

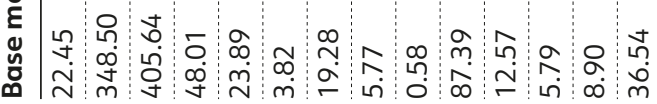


SUPPLEMENTARY TABLE 3 | Differential abundance analysis of bacterial orders following combination of tomato and cotton samples based on infection by wild-type V. dahliae and the VdAve1 deletion mutant.

\begin{tabular}{|c|c|c|c|}
\hline $\begin{array}{l}\text { Average relative } \\
\text { abundance (\%) }\end{array}$ & $\log 2 \mathrm{FC}$ & $p$ value & Order \\
\hline 1.84 & -0.37 & $3.41 \mathrm{E}-03$ & Sphingomonadales \\
\hline 0.08 & -0.71 & $1.76 \mathrm{E}-02$ & Fimbriimonadales \\
\hline 5.30 & 1.05 & $2.35 \mathrm{E}-02$ & Pseudomonadales \\
\hline 13.61 & -0.18 & $3.71 \mathrm{E}-02$ & Sphingobacteriales \\
\hline 8.45 & -0.21 & 7.11E-02 & Xanthomonadales \\
\hline 0.32 & -0.29 & $1.22 \mathrm{E}-01$ & Solirubrobacterales \\
\hline 0.22 & 2.11 & $1.34 \mathrm{E}-01$ & Ktedonobacterales \\
\hline 0.40 & 0.41 & $1.81 \mathrm{E}-01$ & Rhodocyclales \\
\hline 0.14 & 0.36 & $1.83 \mathrm{E}-01$ & Gaiellales \\
\hline 0.25 & 0.38 & $2.02 \mathrm{E}-01$ & Gallionellales \\
\hline 1.30 & 1.54 & $2.10 \mathrm{E}-01$ & Enterobacteriales \\
\hline 11.59 & -0.04 & $2.43 \mathrm{E}-01$ & Burkholderiales \\
\hline 0.01 & -1.36 & $2.78 \mathrm{E}-01$ & Gammaproteobacteria incertae sedis \\
\hline 0.33 & 0.32 & $3.04 \mathrm{E}-01$ & Gp1 \\
\hline 0.02 & -1.22 & $3.07 \mathrm{E}-01$ & Oligoflexales \\
\hline 3.48 & -0.22 & $3.62 \mathrm{E}-01$ & Flavobacteriales \\
\hline 0.09 & 0.44 & $3.73 \mathrm{E}-01$ & Nitrosomonadales \\
\hline 0.36 & 0.39 & $3.88 \mathrm{E}-01$ & Chlamydiales \\
\hline 0.82 & 0.15 & $3.89 \mathrm{E}-01$ & Opitutales \\
\hline 0.12 & 0.24 & 4.07E-01 & Holophagales \\
\hline 1.19 & -0.12 & $4.54 \mathrm{E}-01$ & Verrucomicrobiales \\
\hline 0.03 & -0.78 & 4.86E-01 & Candidatus_Koribacter \\
\hline 0.02 & 0.55 & 4.87E-01 & Methanosarcinales \\
\hline 3.09 & -0.12 & $5.01 \mathrm{E}-01$ & Alphaproteobacteria incertae sedis \\
\hline 0.01 & 1.09 & $5.05 \mathrm{E}-01$ & Rhodobacterales \\
\hline 3.11 & -0.14 & $5.23 \mathrm{E}-01$ & Actinomycetales \\
\hline 0.68 & -0.17 & $5.24 \mathrm{E}-01$ & Bdellovibrionales \\
\hline 0.25 & 0.08 & $5.34 \mathrm{E}-01$ & Armatimonadales \\
\hline 0.00 & 0.89 & $5.37 \mathrm{E}-01$ & Sneathiellales \\
\hline 0.01 & -1.02 & $5.37 \mathrm{E}-01$ & Nitrososphaeraceae \\
\hline 1.84 & 0.12 & $5.58 \mathrm{E}-01$ & Cytophagales \\
\hline 0.11 & -0.40 & $5.59 \mathrm{E}-01$ & Gemmatimonadales \\
\hline 0.41 & -0.12 & $5.62 \mathrm{E}-01$ & Gp3 \\
\hline 0.23 & -0.12 & $5.90 \mathrm{E}-01$ & Candidatus_Solibacter \\
\hline 0.01 & 0.64 & $5.94 \mathrm{E}-01$ & Chthonomonadales \\
\hline 1.55 & -0.07 & $6.14 \mathrm{E}-01$ & Rhodospirillales \\
\hline 0.02 & -0.61 & $6.15 \mathrm{E}-01$ & Tepidisphaerales \\
\hline 0.07 & 0.50 & $6.22 \mathrm{E}-01$ & Neisseriales \\
\hline 0.00 & -0.98 & $6.69 \mathrm{E}-01$ & Methylococcales \\
\hline
\end{tabular}




\begin{tabular}{llll}
\hline $\begin{array}{l}\text { Average relative } \\
\text { abundance (\%) }\end{array}$ & log2FC & p value & Order \\
\hline 0.06 & 0.28 & $6.74 \mathrm{E}-01$ & Bacillales \\
\hline 1.42 & 0.08 & $6.79 \mathrm{E}-01$ & Planctomycetales \\
\hline 0.03 & 0.36 & $6.87 \mathrm{E}-01$ & Terrimicrobium \\
\hline 0.02 & 0.36 & $7.10 \mathrm{E}-01$ & Gp2 \\
\hline 0.31 & -0.36 & $7.12 \mathrm{E}-01$ & Methylophilales \\
\hline 0.00 & 0.82 & $7.14 \mathrm{E}-01$ & Gp6 \\
\hline 0.88 & -0.12 & $7.27 \mathrm{E}-01$ & Chromatiales \\
\hline 0.03 & 0.47 & $7.39 \mathrm{E}-01$ & Acidimicrobiales \\
\hline 0.35 & -0.10 & $7.69 \mathrm{E}-01$ & Spirochaetales \\
\hline 0.86 & 0.05 & $8.15 \mathrm{E}-01$ & Telmatobacter \\
\hline 3.71 & -0.02 & $8.76 \mathrm{E}-01$ & Caulobacterales \\
\hline 0.01 & -0.13 & $8.97 \mathrm{E}-01$ & Lactobacillales \\
\hline 0.01 & 0.13 & $9.13 \mathrm{E}-01$ & Oceanospirillales \\
\hline 1.26 & 0.02 & $9.29 \mathrm{E}-01$ & Myxococcales \\
\hline 0.00 & -0.14 & $9.37 \mathrm{E}-01$ & Rickettsiales \\
\hline 0.95 & -0.02 & $9.47 \mathrm{E}-01$ & Terriglobus \\
\hline 0.00 & 0.08 & $9.68 \mathrm{E}-01$ & Gp14 \\
\hline 0.02 & -0.01 & $9.88 \mathrm{E}-01$ & Edaphobacter \\
\hline 0.15 & -0.01 & $9.93 \mathrm{E}-01$ & Acidobacterium \\
\hline 9.35 & 0.00 & $9.93 \mathrm{E}-01$ & Rhizobiales \\
\hline
\end{tabular}

SUPPLEMENTARY TABLE 4 | DESeq2 output of differential abundance analysis of bacterial orders in synthetic communities treated with demineralized water or $4 \mu \mathrm{M}$ VdAve1.

\begin{tabular}{lllllll}
\hline Base mean & log2FC & IfcSE & stat & p value & Adjusted $\mathbf{p}$ value & Order \\
\hline 561.12 & 9.73 & 0.73 & 13.25 & $4.75 \mathrm{E}-40$ & $5.70 \mathrm{E}-39$ & Bacillales \\
\hline 1466.88 & -1.86 & 0.33 & -5.63 & $1.81 \mathrm{E}-08$ & $1.08 \mathrm{E}-07$ & Flavobacteriales \\
\hline 1346.01 & -1.66 & 0.34 & -4.95 & $7.31 \mathrm{E}-07$ & $2.93 \mathrm{E}-06$ & Sphingobacteriales \\
\hline 22098.42 & 1.54 & 0.33 & 4.72 & $2.39 \mathrm{E}-06$ & $7.16 \mathrm{E}-06$ & Pseudomonadales \\
\hline 319.94 & 1.53 & 0.34 & 4.58 & $4.75 \mathrm{E}-06$ & $1.14 \mathrm{E}-05$ & Xanthomonadales \\
\hline 11.14 & 7.21 & 1.63 & 4.42 & $9.66 \mathrm{E}-06$ & $1.93 \mathrm{E}-05$ & Caulobacterales \\
\hline 41.42 & 2.27 & 0.62 & 3.67 & $2.42 \mathrm{E}-04$ & 0.00041441 & Sphingomonadales \\
\hline 350.44 & 1.07 & 0.34 & 3.15 & $1.61 \mathrm{E}-03$ & 0.0024092 & Burkholderiales \\
\hline 44.69 & -1.41 & 0.65 & -2.18 & $2.91 \mathrm{E}-02$ & 0.038864186 & Rhizobiales \\
\hline 29.92 & -1.41 & 0.74 & -1.91 & $5.67 \mathrm{E}-02$ & 0.068092286 & Actinomycetales \\
\hline 7003.49 & -0.23 & 0.27 & -0.86 & $3.90 \mathrm{E}-01$ & 0.425283762 & Enterobacteriales \\
\hline 7.87 & 0.33 & 1.67 & 0.20 & $8.43 \mathrm{E}-01$ & 0.843090862 & Cytophagales \\
\hline
\end{tabular}


SUPPLEMENTARY TABLE 5 | Effectors of $V$. dahliae strain JR2 with predicted structural homology with known antimicrobial proteins.

\begin{tabular}{|c|c|c|c|c|c|c|}
\hline \multirow{2}{*}{ V. dahliae gene ID } & \multirow{2}{*}{$\begin{array}{l}\text { Predicted structural } \\
\text { homolog }\end{array}$} & \multicolumn{2}{|c|}{ Confidence Coverage } & \multirow{2}{*}{$\begin{array}{l}\text { Size } \\
(\mathrm{kDa})\end{array}$} & \multicolumn{2}{|c|}{ Cysteines Isoelectric } \\
\hline & & (\%) & $(\%)$ & & (\%) & point \\
\hline VDAG_JR2_Chr2g00770 & Aerolysin/toxin & 82.6 & 55 & 23.7 & 4.6 & 5.74 \\
\hline VDAG_JR2_Chr3g05620 & Defensin & 95.6 & 81 & 7 & 9.5 & 6.98 \\
\hline VDAG_JR2_Chr3g12130 & Microbial ribonuclease & 100 & 84 & 10.8 & 3.3 & 8.57 \\
\hline VDAG_JR2_Chr4g10160 & Aerolysin/toxin & 92.7 & 22 & 42 & 2.1 & 4.77 \\
\hline VDAG_JR2_Chr5g07180 & Defensin & 59.9 & 21 & 9.4 & 4.5 & 8.87 \\
\hline VDAG_JR2_Chr5g09210 & Heparin-binding protein & 58.6 & 37 & 21.8 & 2.8 & 4.06 \\
\hline VDAG_JR2_Chr5g09660 & Aerolysin/toxin & 79.4 & 59 & 25.2 & 1.7 & 5.71 \\
\hline VDAG_JR2_Chr6g00350 & Leukocidin & 79.3 & 20 & 38.5 & 2.9 & 4.43 \\
\hline VDAG_JR2_Chr6g03080 & Defensin & 28.1 & 21 & 10.7 & 6.2 & 5.15 \\
\hline VDAG_JR2_Chr8g09620 & Defensin & 75.8 & 20 & 12.2 & 7.1 & 8.99 \\
\hline
\end{tabular}

SUPPLEMENTARY TABLE 6 | Sample statistics 16 S ribosomal DNA profiling.

\begin{tabular}{|c|c|c|c|c|c|}
\hline Sample name & Host & Tissue/sample & Treatment & $\begin{array}{l}\text { Quality } \\
\text { filtered } \\
\text { paired-end } \\
\text { reads }\end{array}$ & $\begin{array}{l}\text { Percentage } \\
\text { of reads } \\
\text { assigned to } \\
\text { microbiome }\end{array}$ \\
\hline SI_Mock_A & Tomato & Roots + rhizosphere & Mock & 41164 & 40.8 \\
\hline SI_Mock_B & Tomato & Roots + rhizosphere & Mock & 48069 & 46.4 \\
\hline SI_Mock_C & Tomato & Roots + rhizosphere & Mock & 43366 & 40.9 \\
\hline SI_WT_A & Tomato & Roots + rhizosphere & V. dahliae WT & 33386 & 41.2 \\
\hline SI_WT_B & Tomato & Roots + rhizosphere & V. dahliae WT & 39518 & 40.1 \\
\hline SI_WT_C & Tomato & Roots + rhizosphere & V. dahliae WT & 43502 & 41.6 \\
\hline SI_ $\Delta$ VdAve1_A & Tomato & Roots + rhizosphere & V. dahliae $\Delta$ VdAve 1 & 51130 & 49.2 \\
\hline SI_ $\triangle$ VdAve1_B & Tomato & Roots + rhizosphere & V. dahliae $\Delta V d$ Ave 1 & 43869 & 43.5 \\
\hline SI_AVdAve1_C & Tomato & Roots + rhizosphere & V. dahliae $\Delta$ VdAve1 & 46159 & 41.3 \\
\hline Gh_Mock_A & Cotton & Roots + rhizosphere & Mock & 93241 & 48.2 \\
\hline Gh_Mock_B & Cotton & Roots + rhizosphere & Mock & 90366 & 46.1 \\
\hline Gh_Mock_C & Cotton & Roots + rhizosphere & Mock & 92144 & 43.8 \\
\hline Gh_WT_A & Cotton & Roots + rhizosphere & V. dahliae WT & 115812 & 49.6 \\
\hline Gh_WT_B & Cotton & Roots + rhizosphere & V. dahliae WT & 105032 & 53.5 \\
\hline Gh_WT_C & Cotton & Roots + rhizosphere & V. dahliae WT & 91601 & 45.9 \\
\hline Gh_ $\triangle \mathrm{VdAve1} A \mathrm{~A}$ & Cotton & Roots + rhizosphere & V. dahliae $\Delta$ VdAve1 & 92975 & 48.1 \\
\hline Gh_ $\Delta \mathrm{VdAve1}$ _B & Cotton & Roots + rhizosphere & V. dahliae $\Delta$ VdAve1 & 118244 & 51.6 \\
\hline Gh_ $\Delta$ VdAve1_C & Cotton & Roots + rhizosphere & V. dahliae $\Delta$ VdAve1 & 112762 & 50.4 \\
\hline SI_Sm_WT_A & Tomato & Stems & V. dahliae WT & 85526 & 3.0 \\
\hline SI_Sm_WT_B & Tomato & Stems & V. dahliae WT & 87981 & 2.5 \\
\hline SI_Sm_WT_C & Tomato & Stems & V. dahliae WT & 71022 & 0.6 \\
\hline SI_Sm_ $\triangle \mathrm{VdAve} 1 \_\mathrm{A}$ & Tomato & Stems & V. dahliae $\triangle \mathrm{VdAve} 1$ & 110437 & 3.2 \\
\hline SI_Sm_AVdAve1_B & Tomato & Stems & V. dahliae $\Delta \mathrm{VdAve} 1$ & 112487 & 1.9 \\
\hline
\end{tabular}




\begin{tabular}{|c|c|c|c|c|c|}
\hline Sample name & Host & Tissue/sample & Treatment & $\begin{array}{l}\text { Quality } \\
\text { filtered } \\
\text { paired-end } \\
\text { reads }\end{array}$ & $\begin{array}{l}\text { Percentage } \\
\text { of reads } \\
\text { assigned to } \\
\text { microbiome }\end{array}$ \\
\hline SI_Sm_AVdAve1_C & Tomato & Stems & V. dahliae $\Delta$ VdAve1 & 115161 & 1.5 \\
\hline Soil_Mock_A & - & Soil & Mock & 27945 & 95.13 \\
\hline Soil_Mock_B & - & Soil & Mock & 30318 & 96.20 \\
\hline Soil_Mock_C & - & Soil & Mock & 28317 & 97.22 \\
\hline Soil_Mock_D & - & Soil & Mock & 28306 & 96.81 \\
\hline Soil_Mock_E & - & Soil & Mock & 29562 & 97.22 \\
\hline Soil_WT_A & - & Soil & V. dahliae WT & 28219 & 94.16 \\
\hline Soil_WT_B & - & Soil & V. dahliae WT & 28559 & 96.37 \\
\hline Soil_WT_C & - & Soil & V. dahliae WT & 28820 & 97.13 \\
\hline Soil_WT_D & - & Soil & V. dahliae WT & 27271 & 94.44 \\
\hline Soil_ $\triangle$ VdAve1_A & - & Soil & V. dahliae $\Delta$ VdAve1 & 26646 & 94.38 \\
\hline Soil_ $\triangle$ VdAve1_B & - & Soil & V. dahliae $\Delta$ VdAve1 & 28351 & 95.27 \\
\hline Soil_ $\triangle$ VdAve1_C & - & Soil & V. dahliae $\Delta$ VdAve 1 & 27018 & 95.27 \\
\hline Soil_ $\triangle$ VdAve1_D & - & Soil & V. dahliae $\Delta$ VdAve1 & 26506 & 90.68 \\
\hline Soil_ $\Delta$ VdAve1_E & - & Soil & V. dahliae $\Delta \mathrm{VdAve} 1$ & 28550 & 96.41 \\
\hline MS_Soil_Mock_A & - & MS medium + soil & Mock & 36355 & 99.93 \\
\hline MS_Soil_Mock_B & - & MS medium + soil & Mock & 38522 & 99.93 \\
\hline MS_Soil_Mock_C & - & MS medium + soil & Mock & 37186 & 99.99 \\
\hline MS_Soil_Mock_D & - & MS medium + soil & Mock & 37218 & 99.93 \\
\hline MS_Soil_Mock_E & - & MS medium + soil & Mock & 38458 & 99.92 \\
\hline MS_Soil_WT_A & - & MS medium + soil & V. dahliae WT & 38802 & 99.94 \\
\hline MS_Soil_WT_B & - & MS medium + soil & V. dahliae WT & 39506 & 99.98 \\
\hline MS_Soil_WT_C & - & MS medium + soil & V. dahliae WT & 37870 & 99.99 \\
\hline MS_Soil_WT_D & - & MS medium + soil & V. dahliae WT & 38522 & 99.94 \\
\hline MS_Soil_WT_E & - & MS medium + soil & V. dahliae WT & 39424 & 100.00 \\
\hline MS_Soil_AVdAve1_A & - & MS medium + soil & V. dahliae $\Delta$ VdAve 1 & 38589 & 99.98 \\
\hline MS_Soil_AVdAve1_B & - & MS medium + soil & V. dahliae $\Delta$ VdAve1 & 38953 & 99.97 \\
\hline MS_Soil_AVdAve1_C & - & MS medium + soil & V. dahliae $\Delta$ VdAve 1 & 38934 & 99.97 \\
\hline MS_Soil_ $\Delta$ VdAve1_D & - & MS medium + soil & V. dahliae $\Delta$ VdAve1 & 39135 & 100.00 \\
\hline MS_Soil_AVdAve1_E & - & MS medium + soil & V. dahliae $\Delta$ VdAve1 & 38984 & 99.99 \\
\hline Syncom_MQ_A & - & Synthetic community & Demineralized water & 28841 & 100.00 \\
\hline Syncom_MQ_B & - & Synthetic community & Demineralized water & 28773 & 100.00 \\
\hline Syncom_MQ_C & - & Synthetic community & Demineralized water & 34298 & 100.00 \\
\hline Syncom_VdAve1_A & - & Synthetic community & Purified VdAve1 & 28407 & 100.00 \\
\hline Syncom_VdAve1_B & - & Synthetic community & Purified VdAve1 & 28471 & 100.00 \\
\hline Syncom_VdAve1_C & - & Synthetic community & Purified VdAve1 & 27752 & 100.00 \\
\hline
\end{tabular}


SUPPLEMENTARY TABLE 7 | Primers used in this study.

\begin{tabular}{|c|c|c|}
\hline Name & Sequence $\left(5^{\prime}-->3^{\prime}\right)$ & Application \\
\hline Xhol_VdAve1_Fw & $\begin{array}{l}\text { CGGTATCTCGAGGATCTAGGGAC } \\
\text { CGCATCCTAC }\end{array}$ & Protein production \\
\hline VdAve1_BamHI_Rv & $\begin{array}{l}\text { CGTCTAG GATCCTCATTATTATATCT } \\
\text { GTCTAAATTCGATGTTGACC }\end{array}$ & Protein production \\
\hline Xhol_VnAve1_Fw & $\begin{array}{l}\text { CGGTATCTCGAGCAATTAGGGAC } \\
\text { CGCATCC }\end{array}$ & Protein production \\
\hline VnAve1_BamHI_Rv & $\begin{array}{l}\text { CGTCTAGGATCCTCATTATTATATCT } \\
\text { GTTCAAACTCG }\end{array}$ & Protein production \\
\hline VdAve1_qPCR_Fw & TGTTACCAAAGCAGCACACAAGG & Real-time PCR \\
\hline VdAve1_qPCR_Rv & CCTTATGCCTCGTTCCCTTCCAC & Real-time PCR \\
\hline VdGAPDH_Fw & CGAGTCCACTGGTGTCTTCA & Real-time PCR \\
\hline VdGAPDH_Rv & CCCTCAACGATGGTGAACTT & Real-time PCR \\
\hline ITS1-Fw & AAAGTTTTAATGGTTCGCTAAGA & Real-time PCR \\
\hline St-Ve1-Rv & CTTGGTCATTTAGAGGAAGTAA & Real-time PCR \\
\hline SIRUB_FW & GAACAGTTTCTCACTGTTGAC & Real-time PCR \\
\hline SIRUB_Rv & CGTGAGAACCATAAGTCACC & Real-time PCR \\
\hline AtRUB_Fw & GCAAGTGTTGGGTTCAAAGCTGGTG & Real-time PCR \\
\hline AtRUB_Rv & CCAGGTTGAGGAGTTACTCGGAATGCTG & Real-time PCR \\
\hline Novosphingobium_Fw & CGGAATAACAGTTAGAAATGACTGC & Real-time PCR \\
\hline Novosphingobium_Rv & CAGGTACTGTCATTATCATCCCT & Real-time PCR \\
\hline Sphingopyxis_Fw & CGGAATAACTCAGAGAAATTTGTGC & Real-time PCR \\
\hline Sphingopyxis_Rv & CCGGTACTGTCATTATCATCCCG & Real-time PCR \\
\hline VdAMP2_qPCR_Fw & CATGGAACACGACCAACTGC & Real-time PCR \\
\hline VdAMP2_qPCR_Rv & GATCCATTCGGGCTTCGACT & Real-time PCR \\
\hline $16 s \_B a c t 340 F$ & TCCTACGGGAGGCAGCAGT & Real-time PCR \\
\hline $16 s \_533 R$ & TTACCGCGGCTGCTGGCAC & Real-time PCR \\
\hline Pacl_VdAMP2_FT_Fw & $\begin{array}{l}\text { CGGTATTTAATTAAATGGCCACCCGCCG } \\
\text { AAC }\end{array}$ & $\begin{array}{l}\text { To generate } \mathrm{pVdAve} 1:: \mathrm{VdAMP2} \\
\text { transformant }\end{array}$ \\
\hline VdAMP2_Notl_RV & $\begin{array}{l}\text { CGTCTAGCGGCCGCTCAGATCGAGAAG } \\
\text { CCGAG }\end{array}$ & $\begin{array}{l}\text { To generate } \mathrm{pVdAve} 1:: \mathrm{VdAMP} 2 \\
\text { transformant }\end{array}$ \\
\hline JR2_VdAMP2_LB_Fw & $\begin{array}{l}\text { GGTCTTAAUAGGCGTAGGGAGGAGAT } \\
\text { GTT }\end{array}$ & To generate VdAMP2 deletion mutant \\
\hline JR2_VdAMP2_LB_Rv & $\begin{array}{l}\text { GGCATTAAUCCATGCTAGGCACAGAC } \\
\text { CAA }\end{array}$ & To generate VdAMP2 deletion mutant \\
\hline JR2_VdAMP2_RB_Fw & $\begin{array}{l}\text { GGACTTAAUATTCGTGTGAAGCCC } \\
\text { TTGGA }\end{array}$ & To generate VdAMP2 deletion mutant \\
\hline JR2_VdAMP2_RB_Rv & $\begin{array}{l}\text { GGGTTTAAUGTTGCAGAAGCCCT } \\
\text { GTTTGG }\end{array}$ & To generate VdAMP2 deletion mutant \\
\hline $27 \mathrm{~F}$ & AGAGTTTGATCCTGGCTCAG & 16S identification \\
\hline $1492 R$ & GGWTACCTTGTTACGACT & 16S identification \\
\hline
\end{tabular}





\section{Chapter 4}

\section{Teichoic acids protect Bacillus subtillis}

against the antimicrobial Verticillium

\section{dahiliae effector ValAvel}

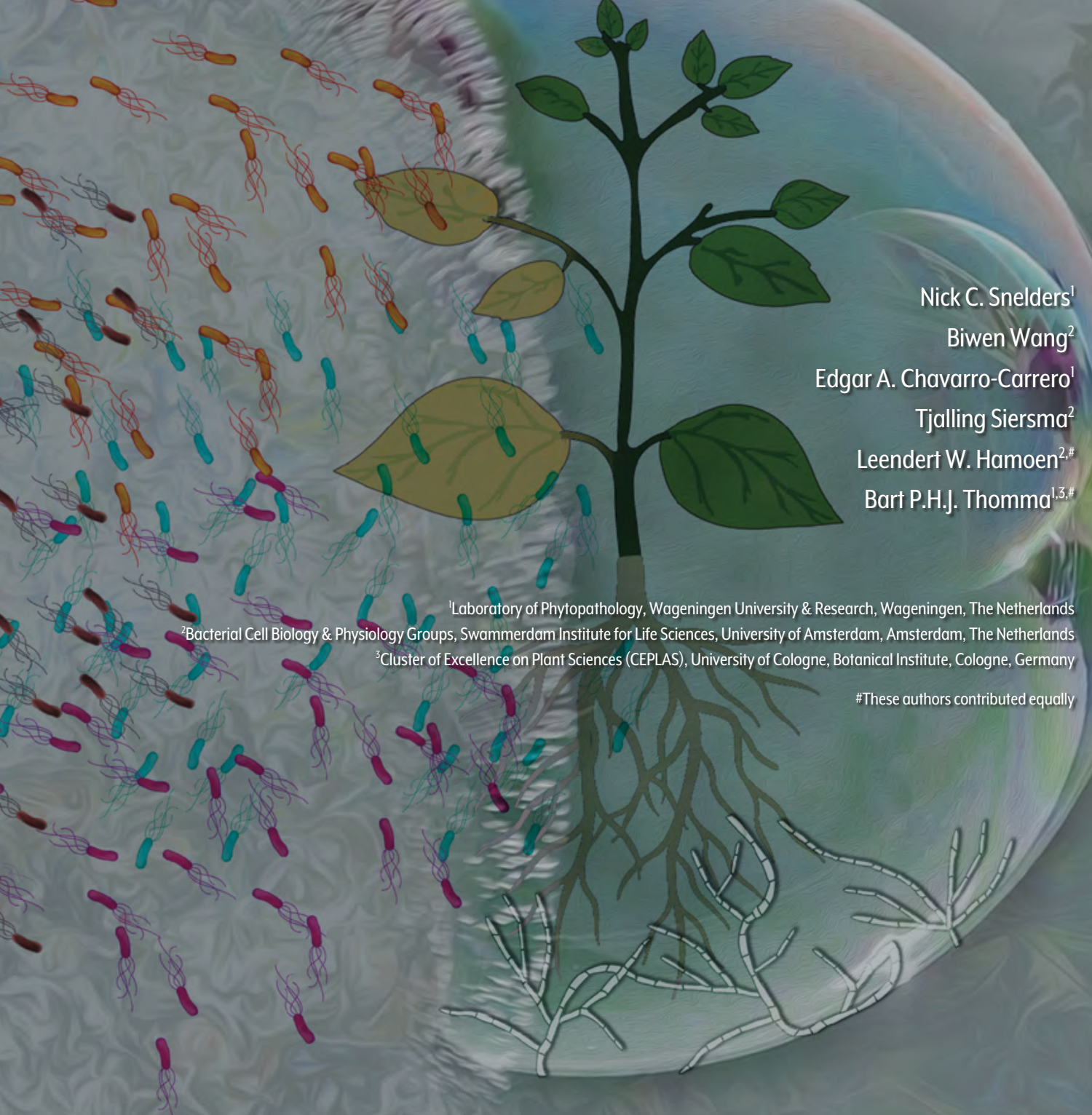




\begin{abstract}
Antimicrobial compounds secreted by a wide diversity of organisms play crucial roles in defense against microbial threats. Free-living microbes are well-known producers of antimicrobials and represent the primary source of clinically used antibiotics. Plantassociated microbes, including pathogens, are also known to secrete a plethora of molecules during host colonization, typically known as effectors, but also during life stages outside the host. The study of effector molecules secreted by plant pathogens has largely been confined to the interaction with their hosts. However, we recently showed that the effector protein VdAve1 that is secreted by the fungal vascular wilt pathogen Verticillium dahliae is a bactericidal protein that promotes host colonization through the active suppression of bacterial antagonists in the plant microbiome. Additionally, we showed that $\mathrm{VdAve} 1$ is also exploited to promote niche colonization in the soil, where the fungus resides in the absence of a host. Thus far, the mode of action underlying the antimicrobial activity of $\mathrm{VdAve} 1$ has remained unknown. In this study, by subjecting Bacillus subtilis to transcriptome profiling and forward genetic analyses, we reveal that similar processes operate in $B$. subtilis in protection against VdAve 1 and against lysozyme, with a central role of the sigV regulon. Furthermore, we show based on these analyses that teichoic acids play a prominent role in mediating tolerance against the effector protein. While $B$. subtilis mutants that are devoid of minor wall teichoic acids ( $\triangle$ galE) or lipoteichoic acids $(\triangle g t a B)$ are severely impaired in growth when exposed to $\mathrm{VdAve} 1$, clear inhibition of $\mathrm{VdAve} 1$ activity can be induced by lipoteichoic acids supplementation, suggesting that teichoic acids directly affect VdAve1 activity.
\end{abstract}




\section{Introduction}

Throughout all domains of life organisms secrete antimicrobial compounds that play crucial roles in defense against microbial threats ${ }^{90,104,105}$. In higher organisms, like animals and plants, antimicrobial proteins or peptides that are collectively termed AMPs constitute important components of the innate immune response that is activated upon detection of microbial invaders ${ }^{106}$. AMPs encompass a very diverse group of generally small proteins that are categorized into different families based on their structures or activities. The overall structures of the various AMP families range from highly flexible and strongly disordered to very tight and stable ${ }^{106,107}$. Interestingly, despite their highly heterogeneous structural nature, AMPs often affect similar cell components or cellular processes ${ }^{108,109}$. For instance, many AMPs disrupt cell membranes of their target organisms to induce lysis, although the precise modes of action differ ${ }^{110}$. Similarly, cellular components involved in processes like DNA, protein and cell wall synthesis represent common targets of AMPs ${ }^{109}$.

Despite the widespread occurrence of AMPs, only few AMP families are conserved across the eukaryotic domain ${ }^{111,112}$. A wide-spread and well-characterized family of AMPs are the so-called cysteine-stabilized $a \beta(\mathrm{CSa} \beta)$ defensins that are found in animals, plants and fungi, many of which display antimicrobial activity ${ }^{113}$. Plant defensins have predominantly been characterized as antifungal proteins that are involved in the protection of various plant tissues and organs ${ }^{114-116}$. For instance, seeds of plants are known to contain various types of AMPs that are released when the seed coat breaks upon germination. These AMPs protect the germling against the wealth of soil microbes that are attracted to the nutrients that are released upon seed coat rupture ${ }^{115}$. However, besides a role in preformed defense, AMPs also act in inducible defense mechanisms. Plants employ a wide array of immune receptors, so-called invasion pattern receptors (IPR), that sense pathogen invasion through the detection of invasion patterns (IPs) to induce appropriate immune responses ${ }^{5}$. In line with a role in inducible plant defense, several genes that encode antimicrobial CSa $\beta$-defensins are induced upon pathogen attack to restrict colonization ${ }^{117-119}$. Consequently, defensins have been recognized as jasmonate- and ethylene induced members of host immune systems that constitute pathogenesis-related protein family $12(\mathrm{PR}-12)^{120,121}$. Unlike animals, plants also secrete a plethora of secondary metabolites besides AMPs to suppress microbial invaders. Like AMPs these can either be pre-formed, referred to as phytoanticipins, or inducible upon pathogen attack, referred to as phytoalexins ${ }^{122,123}$.

Like eukaryotes, also microbes secrete a multitude of antimicrobial compounds to protect themselves from other microbes and to facilitate their establishment within microbial communities ${ }^{124-126}$. Microbes are well-known producers of antimicrobials that, like plants, comprise AMPs as well as secondary metabolites ${ }^{127}$. In fact, the vast majority of the clinically used antibiotics have originally been discovered as 
antimicrobials that are secreted by free-living microbes. For instance, Actinomycetes are filamentous bacteria that mostly thrive on decaying organic matter in soils and aquatic sediments and represent a primary source of the antimicrobial compounds used today ${ }^{128,129}$.

Like free-living microbes, also plant-associated microbes such as microbial plant pathogens secrete a plethora of molecules in their environment. The study of molecules secreted by plant pathogens has largely been limited to the context of the interaction between the host and the pathogen. Plant pathogens are known to secrete so-called "effector" molecules, including proteins, secondary metabolites and small RNAs, that promote host colonization, often by deregulating host immune responses or other elements of host physiology $25,26,130,131$. However, plants are colonized by an overwhelming number of microbes that collectively form their microbiota, which fulfills crucial roles in their performance and health ${ }^{16,76,77}$. Plants modulate their microbiomes, i.e. the microbes, their genomes and the environment ${ }^{6}$, by attracting beneficial microbes that suppress plant pathogens, for instance through antibiosis ${ }^{10,132}$. Consequently, it can be anticipated that pathogens evolved mechanisms to establish themselves in the complex microbiomes of their hosts. Therefore, we previously hypothesized that the suite of effector proteins secreted by plant pathogens in order to host facilitate colonization not only comprises proteins intended for host manipulation, but also proteins to affect plant-associated microbes ${ }^{78}$.

Previously, we identified the effector protein VdAve1 that is secreted during host colonization by the soil-borne fungal plant pathogen Verticillium dahliae as a virulence factor $^{61}$. VdAve1 is a small $(\sim 12 \mathrm{kDa})$ positively charged protein that is predicted to adopt a tight confirmation through two disulfide bonds ${ }^{133}$. We recently revealed that VdAve1 acts as a novel type of antimicrobial protein that promotes host colonization through the manipulation of host microbiome compositions ${ }^{134}$. We furthermore showed that secretion of $\mathrm{VdAve} 1$ by $\mathrm{V}$. dahliae selectively suppresses the proliferation of antagonistic Sphingomonads in planta, which facilitates fungal host colonization in turn, and thus disease development. Importantly, however, in vitro activity assays using the purified effector revealed that the antimicrobial activity of $\mathrm{VdAve} 1$ is not restricted to bacterial isolates belonging to the Sphingomonads. Rather, VdAve1 affects in vitro proliferation of various bacterial species, including the well-studied model species Bacillus subtilis. Scanning electron microscopy revealed that sub-lethal concentrations of VdAve 1 induced lysis of $B$. subtilis cells, suggesting that VdAve1 exerts bactericidal activity $^{134}$.

We previously showed that VdAve1 was horizontally acquired from plants, where the abundantly present homologs are generally annotated as plant natriuretic peptides (PNPs) ${ }^{61}$. PNPs have been reported to be involved in a multitude of processes, many of which are related to plant homeostasis ${ }^{79,80,135}$. Like VdAve1, most proteins in the large family of PNPs are small positively charged proteins that are predicted to adopt a 
tight confirmation that is stabilized through multiple cysteine-bonds. Interestingly, the predicted structure of the PNPs shares no obvious homology with previously identified antimicrobial proteins. Nonetheless, we discovered that AtPNP-A from the model plant Arabidopsis thaliana completely inhibits $B$. subtilis growth at a protein concentration equal to the minimum inhibitory concentration determined for VdAve $1^{134}$. Collectively, these findings suggest that the antimicrobial activity of VdAve1 was horizontally acquired from a plant donor, and that $\mathrm{VdAve} 1$ is a member of a large family of novel antimicrobial proteins. Here, we study the bactericidal activity of VdAve1 in more detail by using diverse and complementary strategies that include transcriptional profiling on $B$. subtilis upon $\mathrm{VdAve} 1$ exposure, screening of a $B$. subtilis library for mutants with altered sensitivity to the protein, and an attempt to generate spontaneous $B$. subtilis mutants with enhanced tolerance by prolonged exposure to the effector. Collectively, we anticipate that these approaches allow us to generate hypotheses concerning the mode of action of $\mathrm{VdAve} 1$.

\section{Results}

\section{VdAvel induces dissipation of $B$. subtilis cell membranes}

Previously, lysis of $B$. subtilis cells was observed through scanning electron microscopy after one hour of incubation with sub-lethal concentrations of VdAve ${ }^{134}$, suggesting that the effector protein exerts bactericidal activity and acts on the cell envelope. However, based on the relatively long exposure to the protein, we could not rule out the possibility that the lysis is not a direct consequence of the effector activity, but rather a secondary effect induced by prolonged exposure to the protein over time. To examine if $\mathrm{VdAve} 1$ directly acts on $B$. subtilis cell membranes we monitored the cellular localization of the cell division regulator protein $M i n D^{136}$ upon incubation of $B$. subtilis with VdAve1. Importantly, localization of MinD relies on the C-terminal amphipathic helix of the protein that requires a membrane potential for the interaction with the cell membrane ${ }^{137,138}$. Consequently, identification of MinD delocalization using fluorescence microscopy following exposure to antibiotics in a MinD-GFP reporter strain has become a well-characterized method for the identification of membrane-destabilizing compounds ${ }^{139,140}$. To assess whether VdAve1 acts on bacterial cell membranes, we exposed the MinD-GFP reporter strain to VdAve1. We furthermore exposed the reporter strain to $\mathrm{VnAve1}$, a close homolog from the non-pathogenic sister species $V$. nubilum that displays $90 \%$ amino acid identity but that does not cause $B$. subtilis lysis ${ }^{134}$, and to the $V$. dahliae effector protein $V d-D$ that is not known to possess antimicrobial

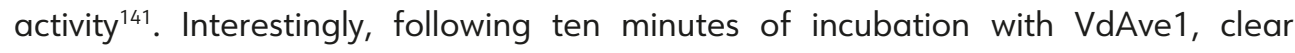
delocalization of MinD-GFP from the cell poles and cell division sites was observed (Fig. 1a). In contrast, exposure to VnAve1 or Vd-D did not lead to MinD-GFP delocalization (Fig. 1a). These findings suggest that VdAve1 acts on bacterial cell membranes, and 
furthermore that the previously observed cell lysis ${ }^{134}$ is a direct consequence of $\mathrm{VdAve} 1$ activity. To reinforce these suggestions, we resuspended an overnight culture of the MinD-GFP reporter strain in phosphate buffered saline to inactivate the bacteria and halt the occurrence of cell divisions, followed by exposure to VdAve1. Also under these conditions lysis of bacterial cells was observed (Fig. 1b), indicating that VdAve1 activity does not require active cell divisions to occur and supporting the activity on bacterial cell membranes as the main mode of action of VdAve1.

a

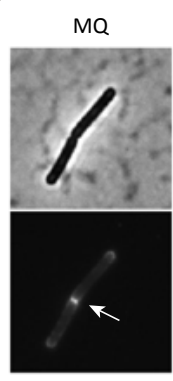

VdAve1

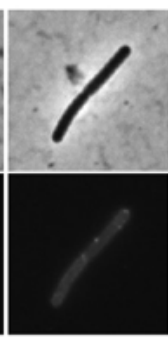

VnAve1

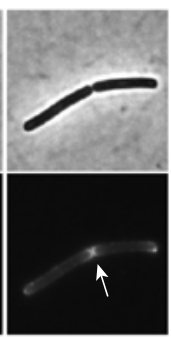

$\mathrm{Vd}-\mathrm{D}$

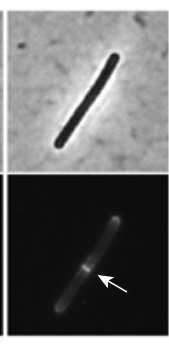

$\overline{5 \mu \mathrm{m}}$ b

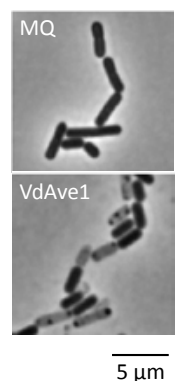

FIGURE 1 | VdAve1 activity induces dissipation of the $B$. subtilis membrane potential and does not require cell division. (a) VdAve1 induces membrane disruption. Localization of MinD-GFP, indicated by arrow heads, in a $B$. subtilis reporter strain upon 10 minutes of incubation in $1 \times$ MIC VdAve 1 with fluorescence microscopy. VdAve1, but not the negative control proteins $V n A v e 1$ and $V d-D$, induced delocalization of MinD, demonstrating that the membrane potential was lost. The top row shows the corresponding bright field images of the bacterial cells. (b) VdAve1 can lyse B. subtilis cells inactivated in phosphate buffered saline (PBS). An overnight culture of the $B$. subtilis reporter strain as used in (a) was resuspended in PBS and incubated with $1 \times$ MIC VdAve1. Clear signs of cell lysis were observed after 30 minutes of incubation, indicating that $\mathrm{VdAve} 1$ activity does not require cell division.

\section{Cations negatively affect VdAvel activity}

Many membrane-active antimicrobial proteins are positively charged molecules that establish ionic interactions with negatively charged target molecules on, or in close proximity of, the cell membrane to exert their activity ${ }^{110}$. Such activities are often accompanied with salt sensitivity, as the presence of positively charged sodium ions impedes the accessibility of the negatively charged target molecules ${ }^{113,142,143}$. Based on its positive charge, we speculated that $\mathrm{VdAve} 1$ similarly requires ionic interactions for its activity. To test this hypothesis, we monitored $B$. subtilis growth inhibition by VdAve1 in the presence of salt or mineral concentrations. Indeed, VdAve1 displayed strong sensitivity for positively charged ions, as increasing concentrations of $\mathrm{Na}^{+}$, or concentrations of $\mathrm{Mg}^{2+}$ and $\mathrm{Ca}^{2+}$ as low as $6.25 \mathrm{mM}$, impaired or completely abolished its activity, respectively (Fig. 2). These findings suggest that VdAve1 indeed targets a negatively charged component of the bacterial cell envelope through ionic interactions. 

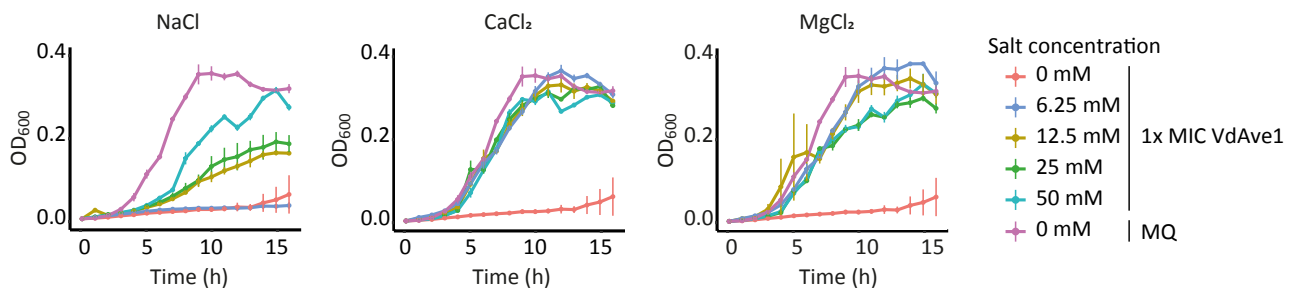

FIGURE 2 | Cations negatively affect VdAve1 activity. The impact of positively charged ions on VdAve1 activity as determined by the ability of the protein to inhibit $B$. subtilis growth in the presence of increasing $\mathrm{NaCl}, \mathrm{CaCl}_{2}$ and $\mathrm{MgCl}_{2}$ concentrations. The controls without salt/minerals and $\mathrm{VdAve} 1$ are included in each graph as red and purple lines. Graphs display the average $\mathrm{OD}_{600}$ of three biological replicates \pm SD.

\section{Transcriptome analyses reveal induction of sigV regulon in B. subtilis following treatment with VdAvel and VnAvel}

Thus far, our findings suggest that the antibacterial activity of $\mathrm{VdAve} 1$ is the consequence of a direct activity on bacterial cell membranes that follows the establishment of an electrostatic interaction with (a) negatively charged component(s) in the bacterial cell envelope. However, this does not yet explain the precise mode of action of the $V$. dahliae effector protein. To improve our understanding of how VdAve1 acts on bacterial cells, we determined transcriptional changes in $B$. subtilis following exposure to sub-lethal concentrations of the protein. To this end, cells were harvested after 5 and 20 minutes of incubation with $4 \mu \mathrm{MVdAve} 1$, which corresponds to $0.5 x \mathrm{MIC}$, to identify early stress responses and subsequent adaptive transcriptomic changes that are induced to facilitate growth in the presence of the protein, respectively. Demineralized water, VnAve1 and the supernatant that was obtained following affinity precipitation of VdAve1 during the purification were included as controls. Principle component analysis of the RNA sequencing data displayed clear separation of the samples based on timepoint (PC1) and treatment (PC2) (Fig. 3a). As anticipated, transcriptome profiles obtained from $B$. subtilis cells treated with $\mathrm{VdAve} 1$ were separated from those treated with supernatant or water, which was most evident after 20 minutes of incubation (Fig. 3a).

Surprisingly, transcriptome profiles obtained following treatment with the presumed negative control VnAve1 clustered closely with those following treatment with VdAve1 (Fig. 3a), revealing that $\mathrm{VdAve} 1$ and $\mathrm{VnAve} 1$ induce similar transcriptional responses in $B$. subtilis. This finding was unanticipated, as previously performed antimicrobial activity assays revealed no inhibitory effect on B. subtilis ${ }^{134}$, and also no MinDGFP delocalization was recorded upon incubation with VnAve1 (Fig 1a). Thus, we reasoned that $\mathrm{VnAve} 1$ may affect $B$. subtilis growth under low salt conditions. Indeed, experimental verification confirmed that $V_{n} A v e 1$ inhibits $B$. subtilis growth at low salt concentrations, albeit with a lower efficacy than VdAve1 (Fig. 3b). Noteworthy, the two proteins displayed differential salt sensitivity as concentrations up to $12 \mathrm{mM} \mathrm{NaCl}$ did not affect VnAve1 while those compromised the activity of VdAve1. 
a

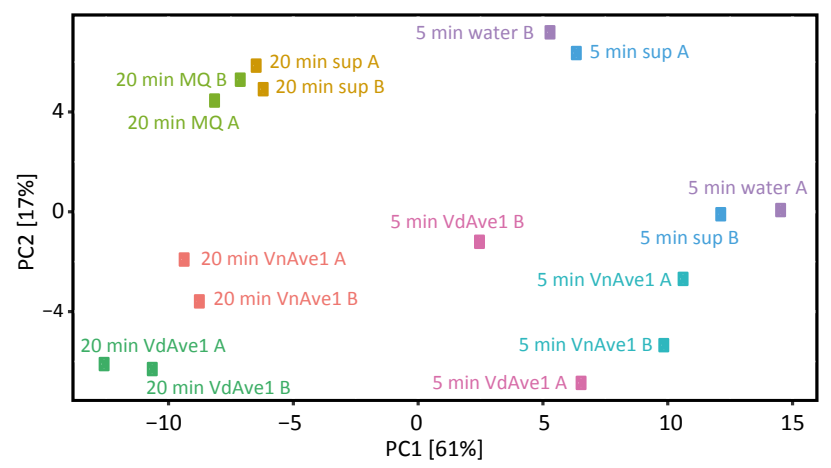

b
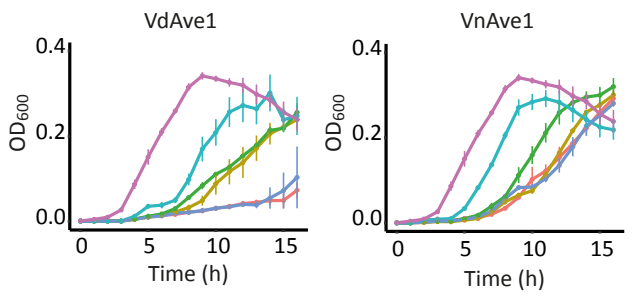

Salt concentration

$+0 \mathrm{mM}$

$+6.25 \mathrm{mM}$

$+12.5 \mathrm{mM}$

$+25 \mathrm{mM}$

$+50 \mathrm{mM}$

$+0 \mathrm{mM} \quad$ I MQ control

FIGURE 3 | VdAve1 and VnAve1 induce similar transcriptomic changes in B. subtilis but display differential efficacy and salt sensitivity. (a) Principle component analysis reveals separation of transcriptomes obtained from $B$. subtilis cells incubated with $4 \mu \mathrm{M}$ VdAve 1 or VnAve1 from cells incubated with supernatant (sup) and water. (b) The impact of sodium ions on VdAve1 and VnAve1 activity as determined by the ability of the proteins to inhibit $B$. subtilis growth in the presence of increasing $\mathrm{NaCl}$ concentrations. The control without salt and effector protein is included in both graphs as a purple line. Graphs display the average $\mathrm{OD}_{600}$ of three biological replicates $\pm \mathrm{SD}$.

To identify transcriptomic changes induced in $B$. subtilis following treatment with both effector proteins when compared with water or supernatant, differential expression analyses were performed. Strikingly, sigV, rsiV, oatA and $y r h K$, representing the complete sigV operon, were the four most highly induced genes at both time points in response to VdAve1 (Table 1) or VnAve1 treatment. SigVencodes the extracytoplasmic function (ECF) sigma factor $V($ SigV). ECF sigma factors regulate gene expression in response to extracellular stresses, including cell envelope active antimicrobial compounds $^{144}$. B. subtilis encodes seven ECF sigma factors in total that, besides SigV, amongst others also include SigW and SigX that are involved in resistance against membrane-active agents, and cationic antimicrobial peptides, respectively ${ }^{144}$. However, neither sigW, nor sigX were induced by VdAve1, which is surprising given the positive charge, salt sensitivity and activity of the effector protein on $B$. subtilis cell membranes. Previously, the sigV regulon was shown to be involved in protection against lysozyme, a widespread antimicrobial enzyme produced by animals that 
is also known as muramidase or $N$-acetylmuramide glycanhydrolase that targets bacterial cell wall peptidoglycan ${ }^{145,146}$. Thus far, induction of the sigV regulon has exclusively been detected in response to lysozyme exposure ${ }^{145}$, and not to any other cell wall damaging agents. These observations suggest that VdAve1 shares particular traits with lysozymes.

TABLE 1 | The ten most highly induced B. subtilis genes following treatment with VdAve1.

\begin{tabular}{|c|c|c|c|c|c|c|c|c|}
\hline \multirow[b]{2}{*}{ Gene } & \multicolumn{4}{|c|}{5 minutes } & \multicolumn{4}{|c|}{20 minutes } \\
\hline & $\begin{array}{c}\text { Log2FC } \\
\text { VdAve1/ } \\
\text { MQ }\end{array}$ & $\begin{array}{l}\text { Adjusted } \\
\text { p value }\end{array}$ & $\begin{array}{c}\text { Log2FC } \\
\text { VnAve1/ } \\
M Q\end{array}$ & $\begin{array}{l}\text { Adjusted } \\
\text { p value }\end{array}$ & $\begin{array}{c}\text { Log2FC } \\
\text { VdAve1/ } \\
M Q\end{array}$ & $\begin{array}{l}\text { Adjusted } \\
\text { p value }\end{array}$ & $\begin{array}{c}\text { Log2FC } \\
\text { VnAve1/ } \\
\text { MQ }\end{array}$ & $\begin{array}{l}\text { Adjusted } \\
\text { p value }\end{array}$ \\
\hline $\operatorname{sig} V$ & 5.04 & $9.08 E-150$ & 4.81 & $2.81 \mathrm{E}-136$ & 6.19 & $5.97 E-186$ & 6.14 & 3.36E-183 \\
\hline rsiV & 4.72 & $2.20 \mathrm{E}-112$ & 4.47 & $8.07 E-101$ & 5.67 & $5.09 E-211$ & 5.62 & $1.96 \mathrm{E}-207$ \\
\hline oat $A$ & 4.26 & $8.48 E-213$ & 3.99 & $1.71 \mathrm{E}-186$ & 5.35 & $0.00 \mathrm{E}+00$ & 5.27 & $0.00 E+00$ \\
\hline$y r h K$ & 4.21 & $1.72 \mathrm{E}-52$ & 3.51 & $3.05 \mathrm{E}-36$ & 4.72 & $8.74 \mathrm{E}-108$ & 4.57 & $3.17 \mathrm{E}-101$ \\
\hline$y m a E$ & 3.84 & $7.59 E-75$ & 3.40 & $2.55 \mathrm{E}-58$ & 2.65 & $1.38 \mathrm{E}-23$ & 2.27 & $5.76 \mathrm{E}-17$ \\
\hline$y m z B$ & 3.70 & $3.45 \mathrm{E}-60$ & 3.40 & $1.88 \mathrm{E}-50$ & 2.36 & $1.78 \mathrm{E}-13$ & 2.10 & $2.37 E-10$ \\
\hline$y d b O$ & 2.11 & $2.21 \mathrm{E}-22$ & 1.94 & $6.72 E-19$ & 2.33 & $1.40 \mathrm{E}-72$ & 2.08 & $2.83 E-57$ \\
\hline maeA & 1.65 & $6.71 \mathrm{E}-06$ & 1.17 & $1.08 \mathrm{E}-13$ & 2.01 & $3.22 \mathrm{E}-22$ & 1.83 & $1.51 E-41$ \\
\hline$m m g D$ & 1.51 & $1.09 \mathrm{E}-14$ & 1.16 & $5.40 \mathrm{E}-6$ & 1.99 & $7.47 \mathrm{E}-09$ & 1.83 & $6.44 \mathrm{E}-07$ \\
\hline bcrC & 1.50 & $1.08 \mathrm{E}-10$ & 0.93 & $3.35 \mathrm{E}-10$ & 1.99 & $2.40 \mathrm{E}-49$ & 1.82 & $1.18 \mathrm{E}-26$ \\
\hline
\end{tabular}

Lysozyme affects bacterial cells through two distinct mechanisms. On one hand it acts as a lytic enzyme that hydrolyzes the $\beta-1,4$ glycosidic linkages between the $N$-acetylmuramic acid (MurNAc) and $N$-acetylglucosamine (GlucNAc) subunits in the peptidoglycan of bacterial cell walls, while on the other hand it acts as an argininerich cationic AMP that perturbs bacterial cell membranes ${ }^{147-149}$. SigV plays a central role in protection of $B$. subtilis against lysozyme as it is responsible for the induction of two crucial operons, the sigV operon it physically resides in itself, as well as the dltABCDE operon ${ }^{144,146}$. The sig $V$ operon comprises four genes, including oat $A$, encoding a peptidoglycan O-acetyltransferase. Peptidoglycan O-acetylation by OatA is a welldescribed cell wall modification that is associated with reduced sensitivity towards the hydrolytic activity of lysozyme in several Gram-positive bacterial species, including B. subtilis ${ }^{146,147,150}$. Additional cell wall modifications involved in protection against lysozyme are facilitated by SigV-mediated induction of the dltABCDE operon that is required for $D$-alanylation of teichoic acids ${ }^{146}$. Teichoic acids are anionic glycopolymers that are ubiquitously present in the peptidoglycan layer of the bacterial cell wall and represent important determinants for resistance or sensitivity to antimicrobial compounds ${ }^{151-154}$. Importantly, D-alanylation reduces the overall negative charge of teichoic acids and is often implicated in resistance to cationic AMPs ${ }^{155-157}$. In line with previous observations made upon treatment with lysozyme, incubation of $B$. subtilis with $\mathrm{VdAve} 1$ and $\mathrm{VnAve} 1$ not only strongly induced the sigVoperon, but also resulted in 
activation of the entire dltABCDE operon (Table 2). Additionally, we detected induction of several other genes involved in teichoic acid biosynthesis or modification, including $\operatorname{tag} F$, tagT, ugtP and yfnl (Table 2). Furthermore, treatment with $\mathrm{VdAve} 1$ and $\mathrm{VnAve} 1$ resulted in strong repression of the DL-endopeptidase genes $c w / O$ and $l y t E$, and induction of the $\mathrm{DL}$-endopeptidase inhibitor gene ise $A$. These transcriptional changes suggest an imbalanced autolysin-mediated de-crosslinking of peptidoglycan, which further supports activity of the effector proteins on the bacterial cell wall. Interestingly, a simultaneous downregulation of des, encoding a phospholipid desaturase, was observed in the presence of VdAve1, suggesting that the activity of the effector protein impacts the membrane and increases membrane fluidity. Finally, VdAve1, and to a lesser extent also $\mathrm{VnAve1}$, induced the expression of several genes involved in cation export, including $c a d A, y d b O, y k k D$ and $n h a K$, suggesting induced leakage of cations into the bacterial cells following membrane dissipation. Alternatively, $B$. subtilis might actively export cations as a defense mechanism to suppress $\mathrm{VdAve} 1$ activity by attempting to impede binding to its negatively charged target(s).

\section{Transposon-sequencing confirms importance of sig $V$ regulon in protection against VdAvel}

To independently verify the importance of the differentially expressed $B$. subtilis genes in protection to VdAve1, and to potentially identify more genes that determine sensitivity to the effector protein, we generated a transposon insertion library and identified mutants with altered sensitivity to $\mathrm{VdAve} 1$ through transposon-sequencing $(\text { Tn-seq })^{158}$. To this end, a pooled $B$. subtilis transposon insertion library was used to inoculate three flasks $\left(O D_{600}=0.1\right)$ containing $6 \mathrm{~mL}$ of low salt LB supplemented with VdAve1, or with ultrapure water (MQ), or the supernatant that was obtained following affinity precipitation of VdAve1 during purification as controls (Supplementary Fig. 1). Once the $\mathrm{OD}_{600}$ of bacterial cells reached 0.8 , which corresponds to three generations of bacterial growth, a sample of $0.75 \mathrm{~mL}$ was transferred to fresh medium, resulting in $\mathrm{OD}_{600}=0.1$. After two transfers to fresh medium, corresponding to a total of nine generations, the bacterial cells were harvested and used to determine the effect of transposon insertion in the different genes on $B$. subtilis proliferation in the presence of VdAve1 when compared with water or supernatant. To this end, the transposon libraries that were subjected to the different treatments were sequenced and the relative occurrence of individual mutants between the treatments was assessed, as mutants that show increased abundance upon VdAve1 treatment point towards the inactivation of genes that mediate sensitivity of $B$. subtilis to the effector protein, whereas mutants with decreased abundance may identify resistance or tolerance genes. 


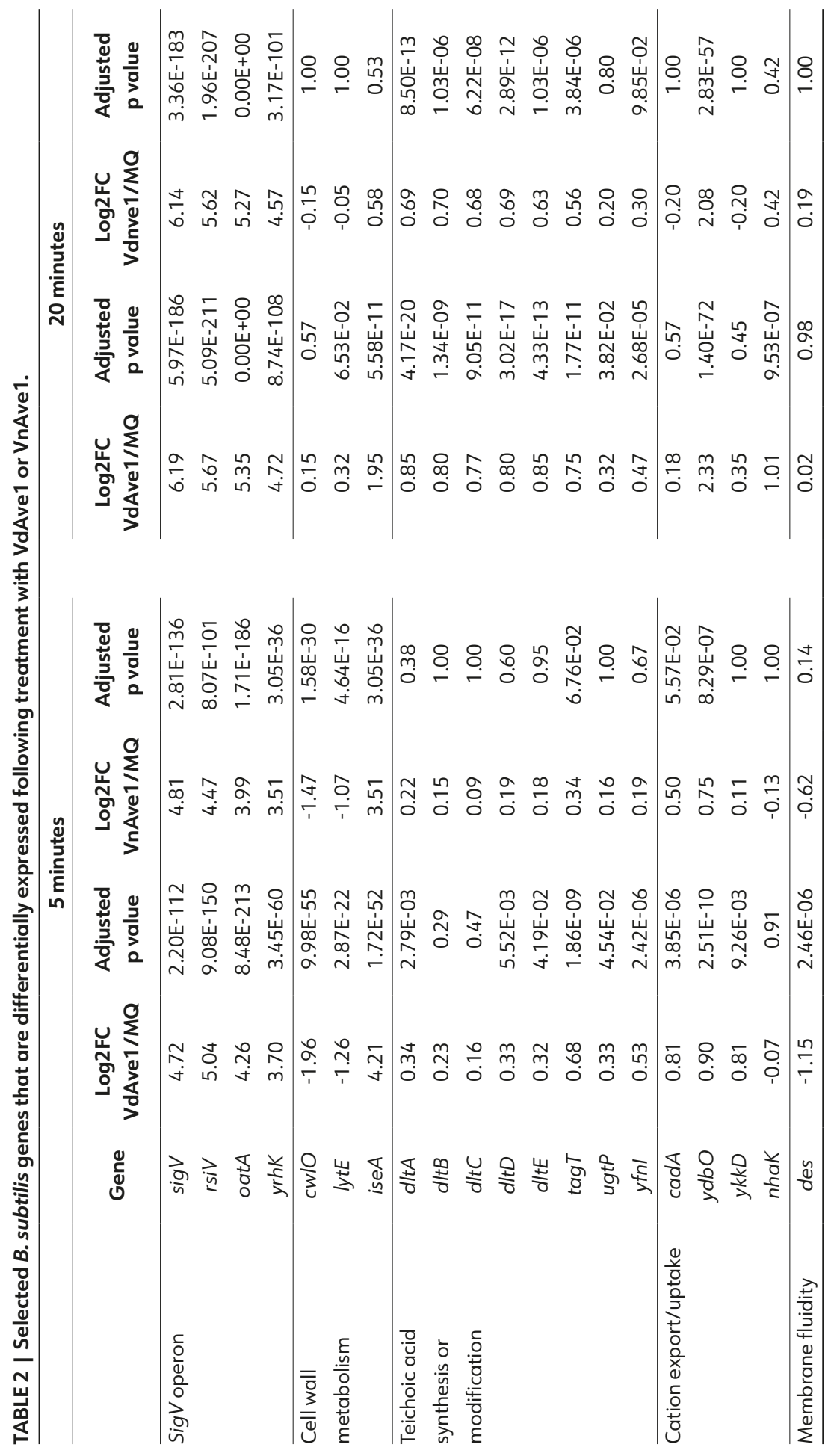


In line with the observed transcriptional changes, mutagenesis of the SigV-regulated genes oat $A$ and $d / t A-E$ enhanced sensitivity to VdAve1 (Table 3). Accordingly, disruption of $r s i V$, encoding the anti-sigma factor responsible for the inactivation of SigV, strongly improved survivability in the presence of $\mathrm{VdAve1.} \mathrm{Moreover,} \mathrm{in} \mathrm{addition}$ to the enhanced sensitivity upon mutagenesis of $d / t A-E$, disruption of several other genes involved in teichoic acid synthesis or modification including gal $E$, gtaB and ugt $P$ also resulted in increased sensitivity to VdAve1. Similarly, inactivation of other genes involved in cell wall integrity or metabolism, such as the peptidase genes $c w l O, \operatorname{dacA}$ and $p b p X$ enhanced sensitivity to VdAve1.

As described above, the disruption of various genes involved in the synthesis or modification of several cell wall components resulted in enhanced sensitivity of $B$. subtilis to VdAve1. However, forward genetic screens, such as our Tn-seq approach, can lead to the discovery of molecular targets of antimicrobial compounds through the identification of mutants with reduced, rather than enhanced, sensitivity. Hence, based on the direct activity of $\mathrm{VdAve} 1$ on the bacterial cell envelope, we queried our Tn-seq output for mutants with increased tolerance to VdAve1 that are affected in the synthesis or modification of cell envelope components. However, no such mutants could be detected. However, inactivation of rsiX, encoding the anti-sigma factor of sigX (Table 3), increased the tolerance of $B$. subtilis to VdAve1, suggesting that also genes belonging to the sig $X$ regulon confer protection against $\mathrm{VdAve} 1$.

TABLE 3 | B. subtilis genes discussed in text that affect sensitivity to VdAve1 as determined through transposon sequencing.

\begin{tabular}{cccccc}
\hline & Gene & $\begin{array}{c}\text { Relative fitness } \\
\text { VdAve1/Supernatant }\end{array}$ & $\begin{array}{c}\text { Adjusted } \\
\text { p value }\end{array}$ & $\begin{array}{c}\text { Relative fitness } \\
\text { VdAve1/MQ }\end{array}$ & $\begin{array}{c}\text { Adjusted } \\
\text { p value }\end{array}$ \\
\hline SigV operon & rsiV & 2.54 & $1.24 \mathrm{E}-17$ & 2.61 & $2.53 \mathrm{E}-18$ \\
& oatA & 0.70 & $7.73 \mathrm{E}-05$ & 0.68 & $1.75 \mathrm{E}-05$ \\
\hline Cell wall & pbpX & $2.07 \mathrm{E}-02$ & $9.60 \mathrm{E}-224$ & $1.90 \mathrm{E}-02$ & $1.56 \mathrm{E}-235$ \\
metabolism & cwlO & $2.73 \mathrm{E}-03$ & $1.65 \mathrm{E}-92$ & $2.66 \mathrm{E}-03$ & $9.25 \mathrm{E}-94$ \\
& dacA & 0.17 & $1.37 \mathrm{E}-24$ & 0.16 & $1.10 \mathrm{E}-26$ \\
\hline Teichoic acid & $d l t A$ & $1.70 \mathrm{E}-03$ & $3.36 \mathrm{E}-298$ & $1.76 \mathrm{E}-03$ & $1.74 \mathrm{E}-292$ \\
synthesis or & $d l t B$ & $9.54 \mathrm{E}-03$ & $0.00 \mathrm{E}+00$ & $9.86 \mathrm{E}-03$ & $0.00 \mathrm{E}+00$ \\
modification & $d l t C$ & $2.09 \mathrm{E}-03$ & $5.06 \mathrm{E}-75$ & $2.52 \mathrm{E}-03$ & $2.47 \mathrm{E}-65$ \\
& $d l t D$ & $2.66 \mathrm{E}-03$ & $0.00 \mathrm{E}+00$ & $2.59 \mathrm{E}-03$ & $0.00 \mathrm{E}+00$ \\
& $d l t E$ & 0.28 & $1.78 \mathrm{E}-22$ & 0.27 & $1.77 \mathrm{E}-24$ \\
& galE & $8.99 \mathrm{E}-02$ & $1.97 \mathrm{E}-146$ & $8.72 \mathrm{E}-02$ & $5.29 \mathrm{E}-150$ \\
& gtaB & 0.21 & $3.66 \mathrm{E}-26$ & 0.24 & $1.60 \mathrm{E}-21$ \\
& $u g t P$ & 0.11 & $3.76 \mathrm{E}-46$ & 0.12 & $4.25 \mathrm{E}-45$ \\
\hline Other genes & greA & 0.15 & $5.54 \mathrm{E}-21$ & 0.16 & $3.41 \mathrm{E}-18$ \\
& miaA & 0.34 & $1.40 \mathrm{E}-06$ & 0.31 & $4.70 \mathrm{E}-08$ \\
& rsiX & 2.50 & $2.72 \mathrm{E}-19$ & 2.36 & $1.06 \mathrm{E}-16$ \\
\hline
\end{tabular}




\section{Adaptation of $B$. subtilis to VdAvel leads to identification of a mutant with increased tolerance}

Whereas screening of the $B$. subtilis transposon insertion library revealed multiple genes that are involved in protection against VdAve1 activity, we did not identify insensitive mutants that may lack molecular targets of the effector protein. Based on our findings thus far, it is conceivable that VdAve1 targets a conserved component of the cell envelope. Possibly, such component is essential for bacterial growth, and hence disruption of genes involved in synthesis of such target through transposon insertion might be lethal. As a complementary approach that allows more subtle mutations to occur and more gradual adaptation to take place, we pursued the generation of tolerant $B$. subtilis strains through continued exposure to $\mathrm{VdAve} 1$ with a gradual increase in concentration in an evolution experiment. To avoid mutations that mediate enhanced expression of extracellular proteases that can degrade the effector protein, we utilized $B$. subtilis strain WB800 that is devoid of eight extracellular proteases ${ }^{159}$. Following preadaptation of $B$. subtilis strain WB800 to the selected growth conditions by repeated transfer to low-salt LB, a sample of the preadapted strain was inoculated into fresh growth medium supplemented with VdAve1 and a subsample of the culture was transferred to fresh medium with VdAve1 every time when the stationary phase of the culture was reached $\left(O D_{600} \geq 2.0\right)$, amounting to a total of six transfers over nine days (Fig. 4a). While the initial VdAve1 concentration to which the culture was exposed was $0.5 x$ MIC, every second transfer the VdAve1 concentration was increased with $0.5 x$ MIC such that after six transfers the bacteria grew in $1.5 x$ MIC. Following the last transfer into 1.5x MIC VdAve1, the liquid culture was plated on agar medium devoid of VdAve1 and five colonies were selected and tested for increased tolerance to VdAve1 by following their growth in medium containing $0.8 \times \mathrm{MIC} \mathrm{VdAve1.} \mathrm{Interestingly,} \mathrm{a} \mathrm{single}$ isolate (\#4) did not display impaired growth in the presence of this concentration, while the other four isolates did not display markedly enhanced tolerance (Fig. 4b). To identify possible mutations that can explain the increased tolerance of isolate 4, we determined genome sequences of the five isolates as well as of the preadapted strain using Oxford Nanopore Technology sequencing. For each strain, minimum $140 \mathrm{Mb}$ of sequence reads were generated, exceeding 30x coverage of the predicted genome size. De novo assemblies using the self-corrected reads resulted in a single circular chromosome for each of the genomes (Supplementary Table 1). As no large-scale structural rearrangements were detected in the genome of isolate 4 when compared with the other genomes (Supplementary Fig. 2), we mined the genome of this isolate for unique smaller polymorphisms, such as indels and SNPs. In total, we identified polymorphisms that affected the coding sequence uniquely in the genome of isolate 4 when compared with the other mutants and the preadapted strain for seven genes only (Table 4). 
a

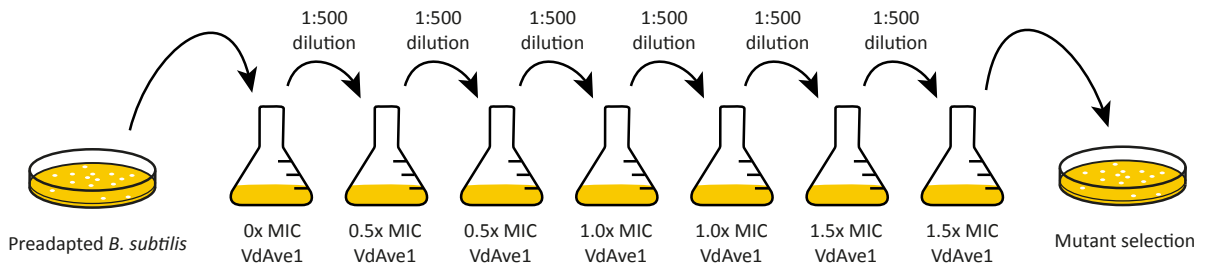

b
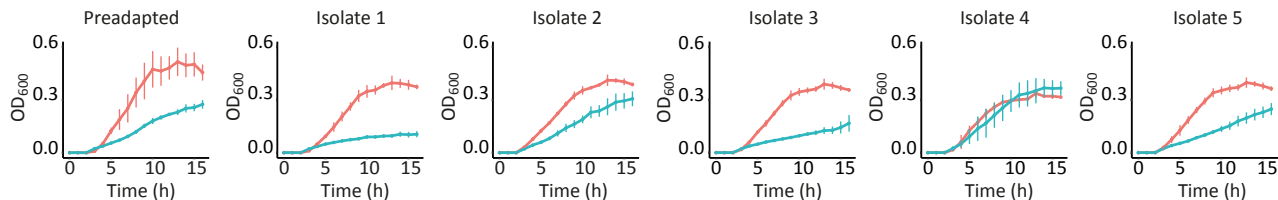

$+\mathrm{MQ}+0.8 \times$ MIC VdAve 1

FIGURE 4 | Adaptation of $B$. subtilis to VdAve1 leads to identification of mutant with increased tolerance. (a) Experimental design of the evolution experiment. A single colony of a preadapted B. subtilis strain was selected from plate and used to prepare a liquid preculture. Subsequently, a sample of the liquid culture was serially transferred into fresh growth medium supplemented with VdAve1 every time when the stationary phase of the culture was reached $\left(\mathrm{OD}_{600} \geq 2.0\right)$. Every second transfer the $\mathrm{VdAve} 1$ concentration to which the bacteria were exposed was increased with $0.5 x$ MIC. (b) Following the last transfer into $1.5 \mathrm{x}$ MIC VdAve1, the liquid culture was plated and five colonies were selected and tested for increased tolerance to $\mathrm{VdAve1}$. Graphs display the average $\mathrm{OD}_{600}$ of three biological replicates \pm SD. Mutant number 4 clearly displayed increased tolerance to the effector protein.

Six out of the seven mutated genes, namely gre $A$, his $B, \operatorname{men} F, \operatorname{mia} A$, yonF and ypw $A$, fulfill intracellular functions that do not immediately reveal an obvious connection with processes in the cell envelope. In order to determine which of the seven genes is responsible for the enhanced tolerance of isolate \#4, we queried our Tn-seq data. Disruption of his $B$, menF, yonF and ypwA through transposon insertion did not alter $B$. subtilis proliferation in the presence of $\mathrm{VdAve1}$. Furthermore, transposon insertion into gre $A$, encoding a transcription elongation factor, or miaA, involved in tRNA modification, increased sensitivity to VdAve1 (Table 3), which may reflect increased or reduced synthesis of proteins that confer a particular degree of protection to the effector. A potentially more interesting mutation was detected in the coding sequence for the UDP-N-acetyl-D-mannosamine transferase TagA, one of the first enzymes in the wall teichoic acid (WTA) biosynthesis pathway. TagA catalyzes the transfer of $\mathrm{N}$-acetylmannosamine (ManNAc) to the C4 hydroxyl of a membrane-anchored $N$-acetylglucosaminyl diphospholipid (GIcNAc-pp-undecaprenyl, lipid I), an essential step in the formation of the conserved WTA linkage unit. Importantly, deletion of tagA completely blocks WTA formation and results in severe defects in cell division and morphology ${ }^{160-162}$. In accordance with such severe growth defects, few to no reads were obtained from mutants with transposon insertions in tagA in our Tn-seq approach. Interestingly, the non-synonymous SNP that was identified in $\operatorname{tag} A$ of isolate 4 did 
introduce an amino acid polymorphism by the replacement of an arginine by a lysine, which is likely to be a more subtle mutation than a gene deletion. Although the precise impact of this mutation on protein function remains enigmatic, the identification of this mutation reinforces the importance of teichoic acids in mediating tolerance to VdAve1 in B. subtilis.

TABLE 4 | Mutated genes identified in B. subtilis isolate 4.

\begin{tabular}{|c|c|c|c|}
\hline Gene & Function/description & Mutation & Localization \\
\hline greA & Transcription elongation factor & Amino acid polymorphism $(F-->L)$ & Intracellular \\
\hline hisB & Metabolism & Amino acid polymorphism $(A-->L)$ & Intracellular \\
\hline menF & Metabolism & Frame shift, premature stop codon & Intracellular \\
\hline $\operatorname{miaA}$ & tRNA modification & Frame shift, premature stop codon & Intracellular \\
\hline $\operatorname{tag} A$ & Teichoic acid biosynthesis & Amino acid polymorphism $(\mathrm{R}-->\mathrm{K})$ & Extracellular \\
\hline yonF & Unknown & Frame shift, premature stop codon & Intracellular \\
\hline уршA & Metalloprotease & Frame shift, premature stop codon & Intracellular \\
\hline
\end{tabular}

\section{Teichoic acids affect VdAvel activity}

The three complementary approaches aimed at identifying factors that determine sensitivity or tolerance of $B$. subtilis to VdAve1 performed in this study, RNAseq, Tn-seq and the evolution experiment, all pointed towards the involvement of teichoic acids in protection against the effector protein. To confirm this observation, we monitored the growth of $B$. subtilis mutants that are devoid of minor wall teichoic acids ( $\triangle$ galE) or lipoteichoic acids $(\Delta g t a B)$ in the presence and absence of VdAve1. In accordance with enhanced sensitivity upon disruption of these genes through transposon insertion (Table 3), both mutants displayed severely impaired growth in the presence of $\mathrm{VdAve} 1$ when compared with the wild-type strain (Fig. 5a).

Teichoic acids can protect bacterial cells against a multitude of antimicrobial compounds, including lysozyme ${ }^{146,147,163}$, while they are also involved in more fundamental processes such as regulation of cell division or cell morphology ${ }^{162}$. Thus, disruption of genes involved in synthesis or modification of teichoic acids can affect overall fitness, leading to increased sensitivity to antimicrobial compounds in turn. To determine whether teichoic acids directly affect VdAve1 activity, we monitored the efficacy of $B$. subtilis growth inhibition by VdAve 1 upon addition of purified lipoteichoic acids. Interestingly, a clear inhibition of $\mathrm{VdAve} 1$ activity was observed in the presence of additional lipoteichoic acids (Fig. 5b), suggesting that teichoic acids directly affect VdAve1 activity. 
a

B. subtilis 168 WT

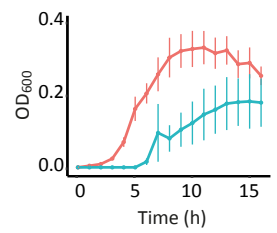

b
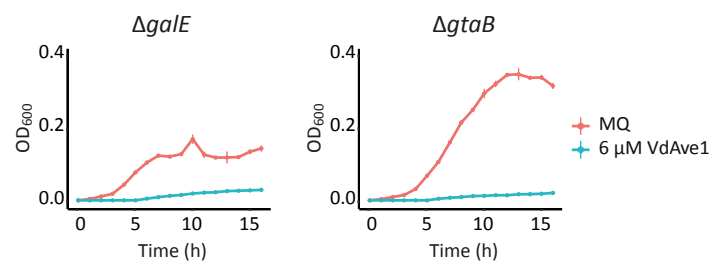

B. subtilis 168 WT

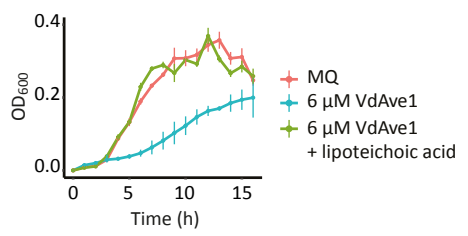

FIGURE 5 | B. subtilis teichoic acids negatively impact VdAve1 activity. (a) B. subtilis mutants devoid of minor wall teichoic acids ( $\triangle g a l E$ ) or lipoteichoic acids $(\triangle g t a B)$ display enhanced sensitivity towards VdAve1 treatment. Graphs display the average $O D_{600}$ of three biological replicates \pm SD. (b) Preincubation of VdAve1 with an equal concentration of lipoteichoic acids purified form $B$. subtilis affects its antimicrobial activity. Graphs display the average $\mathrm{OD}_{600}$ of three biological replicates \pm SD.

\section{Discussion}

Molecules secreted into their environments by organisms of many kinds may represent a valuable source of antimicrobials that may be mined for novel active compounds that can be utilized to treat microbial infections. Till to date, the vast majority of clinically used antibiotics were discovered from free-living saprophytic microbes, many of which are soil-dwelling organisms ${ }^{128}$. Previously, we described that the soilborne plant pathogenic Ascomycete fungus $V$. dahliae exploits the antimicrobial effector proteins $\mathrm{VdAve} 1$ and $\mathrm{VdAMP} 2$ to promote host colonization through the manipulation of microbiome compositions in its environment. Based on the different niches plant pathogens inhabit when compared with most producers of previously described antibiotics, together with the potentially different microbiota compositions that they encounter, plant pathogens are likely to have evolved a different spectrum of antimicrobial activities. Accordingly, we speculated that effectors intended for microbiome manipulation secreted by $V$. dahliae and other plant pathogens might represent an untapped source for novel antibiotics ${ }^{78,134}$. To further support this notion, we studied the antibacterial activity of VdAve 1 in more detail.

Our analyses revealed a surprisingly strong similarity between the $B$. subtilis genes involved in protection against VdAve1 and lysozyme. Intriguingly, VdAve1 is not predicted to carry any enzymatic domain and shares no homology with previously described hydrolytic enzymes, including lysozymes. Nevertheless, transcriptome and 
Tn-seq analyses revealed a central role for the sig $V$ regulon in response to $\mathrm{VdAve} 1$ treatment, a phenomenon that thus far was exclusively described in response to lysozyme and not to any other cell wall-active antimicrobial compounds ${ }^{145}$. Based on its positive charge, its salt sensitivity and its ability to induce dissipation of $B$. subtilis cell membranes, we initially anticipated that $\mathrm{VdAve} 1$ would act as a membrane active AMP. Consequently, the identified similarity with lysozyme, pointing towards the bacterial cell wall as the VdAve 1 target was unanticipated. The highly specific nature of the involvement of the sig $V$ regulon in protection against VdAve1 and lysozyme suggests that VdAve1 acts through hydrolysis of the bacterial cell wall. Importantly, however, altered sensitivities to $\mathrm{VdAve} 1$ as revealed by the $\mathrm{Tn}$-seq still require further validation. Yet, a presumed hydrolytic activity of $\mathrm{VdAve} 1$ is in line with the increased sensitivity as observed for $B$. subtilis upon disruption of oat $A$ by transposon insertion, suggesting that the acetylation of peptidoglycan confers protection to VdAve1, like it does to the hydrolytic activity of lysozyme ${ }^{145,146}$. Moreover, it could also explain the downregulation of the autolysin genes $c w / O$ and $l y t E$ and the simultaneous upregulation of the autolysin inhibitor gene ise $A$, as these transcriptomic changes might be aimed to suppress additional lytic activity in the cell wall in the presence of VdAve1 ${ }^{164,165}$.

Another clear similarity in the response of $B$. subtilis to VdAve 1 and lysozyme involves the biosynthesis and modification of teichoic acids. The three independent approaches adopted in this study collectively pointed towards a crucial role for teichoic acids in the defense of $B$. subtilis against $\mathrm{VdAve1}$, which could be confirmed by testing the growth of $B$. subtilis mutants that are devoid of minor wall teichoic acids ( $\triangle$ galE) or of lipoteichoic acids $(\triangle g t a B)$ in the presence and absence of $\mathrm{VdAve1}$, and by inhibiting VdAve1 activity with purified lipoteichoic acids. This observation fits to the notion that teichoic acids aid in protection against many antimicrobials, including cationic AMPs ${ }^{151,152,154}$. Interestingly, disruption of the genes belonging to the SigV-regulated dlt $A B C D E$ operon, responsible for D-alanylation of teichoic acids, strongly enhanced sensitivity to VdAve1. Modification of teichoic acids by D-alanylation increases the charge of the bacterial cell wall, which, amongst others, affects cationic AMPs by impairing their interaction with the bacterial cell envelope ${ }^{151,157}$. Its positive charge and salt sensitivity suggest that the activity of $\mathrm{VdAve} 1$ depends on an electrostatic interaction with a negatively charged component of the bacterial cell envelope. It therefore seems plausible that D-alanylation of teichoic may acid affects this interaction. The salt sensitivity of VdAve1 is in sharp contrast with that of lysozyme, which is active over a wide range of ionic strengths ${ }^{166}$. Consequently, it seems likely that the protection offered by $D$-alanylation of teichoic acids against lysozyme does not involve the impairment of electrostatic interactions. In that respect it is interesting that disruption of rsiX, encoding the anti-sigma factor of SigX, increased tolerance of $B$. subtilis to $\mathrm{VdAve1,} \mathrm{while} \mathrm{SigX} \mathrm{only} \mathrm{plays} \mathrm{a} \mathrm{marginal} \mathrm{role} \mathrm{in} \mathrm{protection} \mathrm{against}$ 
lysozyme ${ }^{145}$. Although these findings suggest that the $\operatorname{sig} X$ regulon differentially aids in protection against $\mathrm{VdAve} 1$ and lysozyme, it is important to note that the sigX regulon largely overlaps the regulons of other ECFs and, for instance, also comprises the dltABCDE operon ${ }^{144}$. Hence, a potential contribution of SigX to protection against $\mathrm{VdAve} 1$ might, in part, overlap with that of SigV, which may explain why no induction of sigX was observed upon VdAve1 treatment. However, a particular role of genes belonging the $\operatorname{sig} X$ regulon other than $d \mid t A-E$, and a potential similarity between the activities of $\mathrm{VdAve} 1$ and cationic AMPs, cannot be ruled out at this point.

The dissipation of $B$. subtilis cell membranes as detected by delocalization of MinDGFP after 10 minutes of incubation with VdAve1 indicates that bacterial cell lysis is a direct consequence of VdAve1 activity. Unfortunately, the resolution of this assay is rather limited as it only provides insight in the presence or absence of a membrane potential at a certain point in time. A more detailed determination of the VdAve1 killing kinetics, for instance by the real-time quantification of ion or fluorescent dye efflux from the bacterial cells, may provide more insight in the time required to induce lysis. Hydrolytic activity on the bacterial cell wall will gradually induce cell lysis, whereas a cationic AMP-like activity, such as pore-formation, is more likely to induce immediate disruption of cell membranes. Consequently, such determination might help to determine the precise activity of $\mathrm{VdAve1}$. Finally, as a more direct approach, future research can be aimed at the exploration of the potential hydrolytic activity exerted by $V d A v e 1$, for instance by assessing the ability of the effector protein to degrade purified bacterial cell walls or peptidoglycan.

\section{Materials and methods}

Production and purification of recombinant effector proteins. Recombinant VdAve1 and VnAve1 were produced and purified as described previously ${ }^{134}$. As an additional control for the membrane potential measurements and the transcriptome analysis, we pulled down purified VdAve1 stored in demineralized water using 50\% His60 $\mathrm{Ni}^{2+}$ Superflow Resin (Clontech, Mountain View, USA) and pelleted the resinbound protein in order to collect the soluble fraction which we used as "supernatant".

The sequence encoding mature $\mathrm{Vd}-\mathrm{D}$ was cloned into $\mathrm{pET}-15 \mathrm{~b}$ with an $\mathrm{N}$-terminal $\mathrm{His}_{6}$ tag sequence (Novagen, Madison, WI, USA) and the expression vector was transformed into E. coli strain BL21. The BL21 cells were grown in LB medium to an $\mathrm{OD}_{600}=0.8$ at $37^{\circ} \mathrm{C}$ and heterologous protein production was induced with $1 \mathrm{mM} \mathrm{IPTG}$. Following 16 hour of protein production at $28^{\circ} \mathrm{C}$, cells were collected by centrifugation. Subsequently, cells were resuspended in lysis buffer (100 mM Tris, $150 \mathrm{mM} \mathrm{NaCl}, 10 \%$ glycerol, $6 \mathrm{mg} / \mathrm{mL}$ lysozyme (Sigma, St. Louis, MO, USA), $2 \mathrm{mg} / \mathrm{mL}$ deoxycholic acid, $0.06 \mathrm{mg} / \mathrm{mL}$ DNasel, protease inhibitor cocktail (Roche, Mannheim, Germany) and 
incubated for 1 hour at $4^{\circ} \mathrm{C}$. The soluble fraction was separated from the insoluble fraction by centrifugation (30 minutes at 20,000 $\mathrm{g}$ ) ) and subjected to metal affinity chromatography using 50\% His60 Ni2+ Superflow Resin (Clontech, Mountain View, USA) to facilitate purification of the $\mathrm{His}_{6}$-tagged proteins. Purified proteins were dialyzed against demineralized water.

MinD delocalization assay. The MinD delocalization assay was performed as described previously with slight modifications ${ }^{139,140}$. B. subtilis strain TB35 (Supplementary Table 1) expressing MinD-GFP was grown in 0.5x YT ( 4 g/L Tryptone, $2.5 \mathrm{~g} / \mathrm{L}$ Yeast Extract, $1.25 \mathrm{~g} / \mathrm{L} \mathrm{NaCl}$ ) supplemented with $0.1 \%$ xylose at $30^{\circ} \mathrm{C}$ until $\mathrm{OD}_{600}=0.1$. Subsequently, the bacteria were incubated for 10 minutes with $8 \mu \mathrm{M}$ (1x MIC) VdAve1 and immobilized on a thin film of $1 \%$ agarose. Localization of MinD-GFP was determined using an Olympus BX 50 microscope equipped with a Photometrics CoolSNAP fx digital camera.

VdAve1 activity on non-dividing cells. An overnight culture of $B$. subtilis strain TB35 was resuspended in $0.5 x$ PBS $\mathrm{pH} 7.4$ supplemented with $8 \mu \mathrm{M}$ (1x MIC) VdAve1 and incubated for 30 minutes at $30^{\circ} \mathrm{C}$. Pictures were taken using an Olympus BX 50 microscope equipped with a Photometrics CoolSNAP fx digital camera.

In vitro B. subtilis growth assays. The different B. subtilis strains (Supplementary Table 1) were grown on lysogeny broth agar (LBA) at $28^{\circ} \mathrm{C}$. Single colonies were selected and grown overnight in low salt LB (10 g/L Tryptone, $5 \mathrm{~g} / \mathrm{L}$ Yeast Extract, 0.5 $\mathrm{g} / \mathrm{L} \mathrm{NaCl}$ ) at $28^{\circ} \mathrm{C}$ while shaking at $200 \mathrm{rpm}$. Overnight cultures were resuspended to $\mathrm{OD}_{600}=0.05$ in LB low salt LB supplemented with VdAve1 and/or $\mathrm{NaCl}, \mathrm{CaCl}_{2}$ and $\mathrm{MgCl}_{2}$. To determine the impact of lipoteichoic acids on $\mathrm{VdAve1}$ activity, the effector was preincubated for 30 minutes at room temperature with an equal concentration of commercially available B. subtilis lipoteichoic acids (Sigma-Aldrich, St. Louis, MO, USA) prior to addition to the growth medium. For all experiments, $200 \mu \mathrm{L}$ of the different bacterial suspensions was aliquoted in clear 96 well flat bottom polystyrene tissue culture plates and bacterial growth was monitored at $25^{\circ} \mathrm{C}$ as described previously ${ }^{134}$.

Transcriptome analysis. To allow activation of $B$. subtilis $168,10 \mathrm{~mL}$ of fresh low salt LB (10 g/L Tryptone, $5 \mathrm{~g} / \mathrm{L}$ Yeast Extract, $0.5 \mathrm{~g} / \mathrm{L} \mathrm{NaCl}$ ) was inoculated with $100 \mu \mathrm{L}$ of an B. subtilis 168 overnight culture, and incubated at $30^{\circ} \mathrm{C}$ at $200 \mathrm{rpm}$. Next, the activated culture was used to inoculate $10 \mathrm{~mL}$ of low salt LB in multiple $100 \mathrm{~mL}$ flasks to a final $\mathrm{OD}_{600}=0.05$, and bacterial suspensions were allowed to grow at $30^{\circ} \mathrm{C}$ while shaking at $200 \mathrm{rpm}$. Once the cultures reached $\mathrm{OD}_{600}=0.3, M Q, \operatorname{VdAve} 1(4 \mu \mathrm{M}), \mathrm{VnAve}(4$ $\mu M)$ and the supernatant obtained after affinity precipitation of VdAve1, were added to the bacterial suspensions. Following 5 and 20 minutes of incubation at $200 \mathrm{rpm}$ and $30^{\circ} \mathrm{C}, 0.5 \mathrm{~mL}$ of the bacterial cultures was transferred to $2 \mathrm{~mL}$ screw-cap tubes containing $1.5 \mathrm{~g}$ glass beads $(\varnothing 0.1 \mathrm{~mm}), 500 \mu \mathrm{L}$ phenol-chloroform-isoamyl alcohol (25:24:1) and $50 \mu \mathrm{L} 10 \%$ SDS. The samples were mixed and snap-frozen in liquid 
nitrogen. The ice-cold samples were subjected to bead beating (10x for 30 seconds at 6,000 rpm) to ensure lysis of the bacterial cells. Next, tubes were centrifuged for 5 minutes at $13,000 \times \mathrm{g}$ and the aqueous layer was transferred to a new tube containing $400 \mu \mathrm{L}$ of chloroform. Samples were mixed and centrifuged again for 5 minutes at $13,000 \times \mathrm{g}$ after which the aqueous layers were collected and RNA was precipitated using ethanol and sodium acetate. Pellets were collected by centrifugation (30 minutes at $13,000 \times g$ ), resuspended in $M Q$ and treated with DNase. Next, ribosomal RNA depletion was performed on the total RNA using the MICROBExpress ${ }^{\text {TM }}$ Bacterial mRNA Enrichment Kit (Thermo Fisher Scientific, Carlsbad, CA, USA) and sequencing libraries were prepared using the NEBNext ${ }^{\oplus}$ Ultra $^{\text {TM }}$ II Directional RNA Library Prep Kit for Illumina ${ }^{\circ}$ according to the manufacturer's instructions (New England Biolabs, Ipswich, MA, USA). The libraries were sequenced on the Illumina NextSeq 550 platform using NextSeq 500/550 High Output v2.5 kit (75-bp read length). Data analysis was performed on the web-based platform Galaxy. Differential expression analyses were performed using DESeq2 (Galaxy Version 2.11.40.6+galaxy1) ${ }^{101}$.

Transposon sequencing. Construction of the $B$. subtilis transposon insertion library and the transposon sequencing library was performed as described previously with slight modifications ${ }^{167,168}$. For preparation of the transposon insertion library, $6 \mu \mathrm{g}$ of genomic DNA isolated from $B$ subtilis strain BSB1 was mixed with $2 \mu \mathrm{g}$ of transposonharboring plasmid $\mathrm{pCJ} 4$ and $0.4 \mu \mathrm{g}$ of mariner transposase Himar1-C9, in $9.5 \%$ glycerol, $93.5 \mathrm{mM} \mathrm{NaCl}, 9.5 \mathrm{mM} \mathrm{MgCl}{ }_{2}, 3.6 \mu \mathrm{M} \mathrm{BSA}, 1.9 \mathrm{mM}$ dithiothreitol, $20.5 \mathrm{mM}$ HEPES pH 7.9 and incubated overnight at $30^{\circ} \mathrm{C}$ to allow transposition. Subsequently, the DNA was precipitated, purified and resuspended in $15 \mu \mathrm{L} 10 \mathrm{mM} \mathrm{MgCl} \mathrm{2}^{\prime} 1 \mathrm{mM}$ dithiothreitol, $50 \mathrm{mM}$ Tris- $\mathrm{HCl} \mathrm{pH} 7.8$ and mixed with $4 \mu \mathrm{L} 2.5 \mathrm{mM}$ dNTPs and $0.5 \mu \mathrm{L}$ T4 DNA Polymerase (New England Biolabs, Ipswich, MA, USA) and incubated for 20 min at $12^{\circ} \mathrm{C}$ to allow repair of transposon insertion junctions. Next, following heat inactivation of the DNA polymerase, $0.2 \mu \mathrm{L} 2.6 \mathrm{mM}$ NAD and $0.5 \mu \mathrm{L}$. coli DNA ligase (New England Biolabs, Ipswich, MA, USA) was added to the DNA and the mix was incubated overnight at $16^{\circ} \mathrm{C}$ to allow repair of the nicked DNA strands after which the transposon-inserted genomic DNA (Tn-gDNA) was transformed into competent B subtilis BSB1 cells. Approximately 250.000 transformants were selected on plate, pooled in liquid medium and eventually stored at $-80^{\circ} \mathrm{C}$.

The experimental setup for the Tn-seq experiment involving the different treatments, transfer of the bacterial cultures and the total number of generations is shown in supplementary Figure 1. Genomic DNA isolated from the bacterial cells that underwent the different treatments was digested using Mmel (New England Biolabs, Ipswich, MA, USA) and subjected to calf intestinal phosphatase treatment (New England Biolabs, Ipswich, MA, USA) after which the DNA fragments were purified. Meanwhile, a customized Illumina sequencing R1 adapter was annealed to complementary oligonucleotides BW-TnR1-S and BW-TnR1-AntiS (Supplementary Table 3) and ligated 
to the purified DNA fragments using T4 DNA ligase (New England Biolabs, Ipswich, MA, USA). Next, the adapter-ligated DNA fragments were purified using AMPure XP beads (Beckman Coulter, Indianapolis, IN, USA ) and used as template for the generation of Illumina sequencing libraries by PCR amplification using the universal BW-TnPCR-Uni primer and a specific barcoded primer (Supplementary Table 3). The PCR-enriched Tnseq libraries were then subjected to gel electrophoreses using a native $8 \%$ acrylamide gel and DNA fragments of approximately $165 \mathrm{bp}$ were purified from gel and resuspended in 0.1X TE. Finally, the libraries were sequenced on the Illumina NextSeq 550 platform using NextSeq 500/550 High Output v2.5 kit (75-bp read length).

The obtained reads were processed using Python and the web-based platform Galaxy. Briefly, reads were filtered and adapters were trimmed using Trimmomatic (Galaxy Version 0.36.5) ${ }^{169}$. Next, Cutadapt (Galaxy Version 1.16.5) ${ }^{170}$ was used to remove the inverted repeats of the transposon from the $3^{\prime}$ end of the quality filtered and trimmed reads. Subsequently, reads of 13-18 bp were selected and mapped to the $B$. subtills reference genome (NC_000913) using Bowtie2 (Galaxy Version 2.3.4.3+galaxy0) ${ }^{171}$. Next, the customized map_functions was used to extract all TA dinucleotide sequences from gene coding regions in the reference genome, leading to the identifying of in total 183963 TA dinucleotide sequences. Next, we determined the number of reads obtained per TA dinucleotide sequence for the different treatments. On average we obtained 9.53 million reads per library, corresponding to 112457 TA dinucleotide sequences in 4280 coding sequences and an average of 85 reads/insertion.

The impact of transposon insertion in the different genes on $B$. subtilis proliferation under the different experimental conditions was determined as follows. The total number of TA dinucleotide sequences in each coding sequence was used to estimate the number of reads that would be obtained per gene. Next, for each gene we determined the ratio between the estimated and the actual number of reads that were obtained. Finally, statistically significant differences between these ratios across the different experimental conditions were identified using an unpaired two-sided student's t-test.

Evolution experiment. Prior to the prolonged exposure to VdAve1, B. subtilis strain WB800, devoid of eight extracellular proteases ${ }^{159}$, was preadapted to the selected growth conditions. To this end, a single colony of $B$. subtilis strain WB800 was selected from plate and used to inoculate a flask containing liquid low-salt LB $(8.5 \mathrm{mM} \mathrm{NaCl})$. The bacterial culture was allowed to grow overnight at $28^{\circ} \mathrm{C}$ while shaking at $160 \mathrm{rpm}$. The next morning, the overnight culture was used to inoculate fresh growth medium (1:500 dilution) and the bacterial culture was again incubated overnight. Following two weeks of repeated transferring of the overnight cultures into fresh growth medium, the last overnight culture was plated and a single colony was selected for the evolution experiment. Subsequently, the preadapted strain was subjected to prolonged exposure to increasing VdAve1 concentrations according to the experimental setup 
as shown in Figure 4a. Following the last transfer, the liquid culture was plated and five colonies were tested for increased tolerance to VdAve1 and selected for genomic DNA isolation.

Quality, size and quantity of the high molecular weight DNA isolated from the selected isolates was assessed by Nanodrop, gel electrophoresis and Qubit analyses, respectively. A total of $400 \mathrm{ng}$ high molecular weight genomic DNA was barcoded for each isolate using the Rapid Barcoding Kit (SQK-RBK004) (Oxford Nanopore Technologies, Oxford, UK) according to manufacturer's instructions after which the libraries were pooled and sequenced using a R9.4.1 flow cell (Oxford Nanopore Technologies, Oxford, UK). Demultiplexing and base calling of the obtained reads was done using Guppy (version 3.1.5; Oxford Nanopore Technologies, Oxford, UK). Next, barcode and adapter sequences were trimmed using Porechop (version 0.2.4 with default settings) and the reads were self-corrected using Canu (Version 1.8) ${ }^{172}$. Finally, circular chromosomes of the isolates were assembled de novo using Flye (Version 2.7.1; Kolmogorov et. al., 2019). To identify large rearrangements in the genomes of the different isolates, whole genome alignments were performed using Mummer3 (setting: nucmer-maxmatch) ${ }^{174}$. Finally, Mummer3 was also used to identify smaller polymorphisms, such as SNPs and small indels (setting: nucmer -prefix show-snps) $)^{174}$.

\section{Acknowledgements}

B.P.H.J.T. acknowledges support from the Research Council Earth and Life Sciences (ALW) of the Netherlands Organization of Scientific Research (NWO) and from the Deutsche Forschungsgemeinschaft (DFG, German Research Foundation) under Germany's Excellence Strategy - EXC 2048/1 - Project ID: 390686111. Funding for B.W. was provided by a China Scholarship Council fellowship.

\section{Data and materials availability}

The sequencing data have been deposited in the European Nucleotide Archive (ENA) under accession number PRJEB38296. 


\section{Supplementary data}

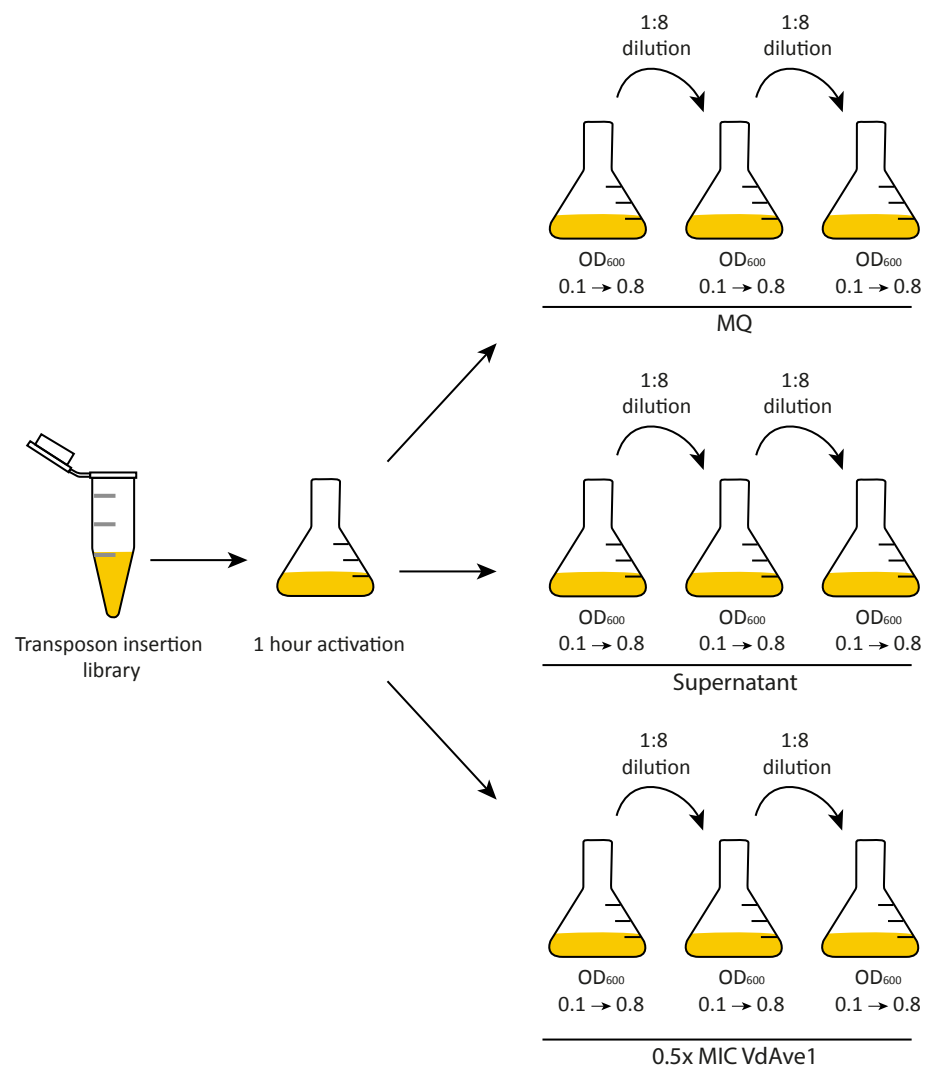

SUPPLEMENTARY FIGURE 1 | Experimental design for the screening of the transposon library. A pooled transposon insertion library was used to activate a single culture in liquid growth medium. After one hour, the activated culture was used to inoculate three flasks containing $6 \mathrm{~mL}$ of fresh growth medium supplemented with $M Q, 4 \mu M V d A v e 1$ or the supernatant obtained following affinity precipitation of $V d A v e 1$ during the purification procedure. Once the bacterial cultures reached $\mathrm{OD}_{600}=0.8, a 0.75 \mathrm{~mL}$ sample of the culture was transferred to a new flask containing fresh growth medium. After two transfers, corresponding to a total of nine generations, the bacterial cells were collected and genomic DNA was isolated. 

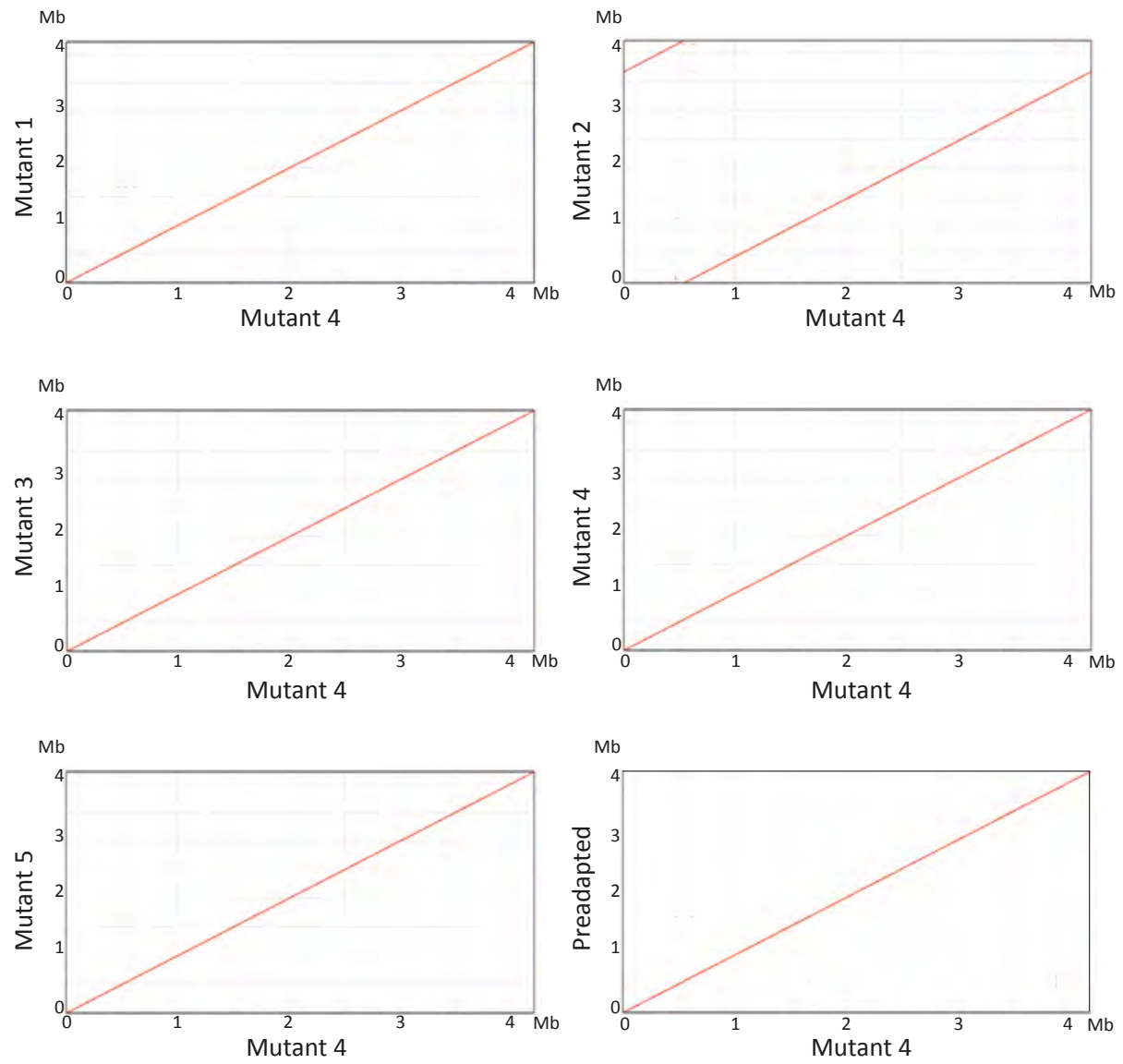

SUPPLEMENTARY FIGURE 2 | Whole genome alignments between $B$. subtilis mutant 4 and the other mutants and the preadapted strain. No large-scale structural rearrangements are observed in the genome of mutant 4 when compared with the other genomes.

SUPPLEMENTARY TABLE 1 | Assembly statistics of sequenced $B$. subtilis strains that were selected after continued exposure to VdAve1.

\begin{tabular}{cccccc}
\hline Strain & Generated data $(\mathrm{Mb})$ & Assembly size $(\mathrm{Mb})$ & \# of contigs & Circular & BUSCO (\%) \\
\hline Isolate 1 & 525 & 4.2 & 1 & Yes & 82.3 \\
\hline Isolate 2 & 399 & 4.2 & 1 & Yes & 80.4 \\
\hline Isolate 3 & 240 & 4.2 & 1 & Yes & 82 \\
\hline Isolate 4 & 470 & 4.2 & 1 & Yes & 84 \\
\hline Isolate 5 & 193 & 4.2 & 1 & Yes & 83.1 \\
\hline Preadapted & 143 & 4.2 & 1 & Yes & 80.4 \\
\hline
\end{tabular}


SUPPLEMENTARY TABLE 2 | B. subtilis strains used in this study.

\begin{tabular}{|c|c|c|c|}
\hline Strain & Genotype & Induction & Reference \\
\hline 168 & $\operatorname{trpC2}$ & - & 175 \\
\hline TB35 & amy::spc Pxyl-gfp-minD & $0.1 \%$ xylose & 139 \\
\hline HM1365 & $\begin{array}{l}\operatorname{trpC2} \text { pgsA' } \Omega(P x y l-p g s A \text { cat }) \\
\text { aprE::(Pspac-Pxyl-xylR lacl erm) }\end{array}$ & $0.1 \%$ xylose & 176 \\
\hline BKE35670 & trpC2 $\Delta$ gtaB::erm & - & 177 \\
\hline BKE38860 & $\operatorname{trpC2} \Delta$ galE::erm & - & 177 \\
\hline WB800 & nprE aprE epr bpr mpr::ble nprB::bsr $\Delta$ vpr wprA::hyg & - & 159 \\
\hline
\end{tabular}

SUPPLEMENTARY TABLE 3 | Primers used in this study.

\begin{tabular}{|c|c|c|}
\hline Name & Sequence $\left(5^{\prime}-->3^{\prime}\right)$ & Application \\
\hline BW-TnR1-S & TCTTTCCCTACACGACGCTCTTCCGATCTNN & Tn-seq library preparation \\
\hline BW-TnR1-Antis & AGATCGGAAGAGCGTCGTGTAGGGAAAGA & Tn-seq library preparation \\
\hline BW-TnPCR-Uni & $\begin{array}{l}\text { AATGATACGGCGACCACCGAGATCTACACTCTTTCCCTA } \\
\text { CACGACGCTCTTCCGATCT }\end{array}$ & Tn-seq library preparation \\
\hline BW-TnPCR-BC43 & $\begin{array}{l}\text { CAAGCAGAAGACGGCATACGAGATGCTGTAGTGACTG } \\
\text { GAGTTCAGACGTGTGCTCTTCCGATCAGACCGGGGACT } \\
\text { TATCATCCAACCTGT }\end{array}$ & Tn-seq library preparation \\
\hline BW-TnPCR-BC44 & $\begin{array}{l}\text { CAAGCAGAAGACGGCATACGAGATATTATAGTGACTG } \\
\text { GAGTTCAGACGTGTGCTCTTCCGATCAGACCGGGGACT } \\
\text { TATCATCCAACCTGT }\end{array}$ & Tn-seq library preparation \\
\hline BW-TnPCR-BC45 & $\begin{array}{l}\text { CAAGCAGAAGACGGCATACGAGATGAATGAGTGACTG } \\
\text { GAGTTCAGACGTGTGCTCTTCCGATCAGACCGGGGACT } \\
\text { TATCATCCAACCTGT }\end{array}$ & Tn-seq library preparation \\
\hline BW-TnPCR-BC46 & $\begin{array}{l}\text { CAAGCAGAAGACGGCATACGAGATTCGGGAGTGACTG } \\
\text { GAGTTCAGACGTGTGCTCTTCCGATCAGACCGGGGACT } \\
\text { TATCATCCAACCTGT }\end{array}$ & Tn-seq library preparation \\
\hline BW-TnPCR-BC47 & $\begin{array}{l}\text { CAAGCAGAAGACGGCATACGAGATCTTCGAGTGACTG } \\
\text { GAGTTCAGACGTGTGCTCTTCCGATCAGACCGGGGACT } \\
\text { TATCATCCAACCTGT }\end{array}$ & Tn-seq library preparation \\
\hline BW-TnPCR-BC48 & $\begin{array}{l}\text { CAAGCAGAAGACGGCATACGAGATTGCCGAGTGACTG } \\
\text { GAGTTCAGACGTGTGCTCTTCCGATCAGACCGGGGACT } \\
\text { TATCATCCAACCTGT }\end{array}$ & Tn-seq library preparation \\
\hline
\end{tabular}





\begin{abstract}
Lysozymes are functionally analogous antimicrobial enzymes that target the bacterial cell wall polymer peptidoglycan by hydrolyzing the $\beta-1,4$ glycosidic bonds between the $N$-acetylmuramic acid and $N$-acetylglucosamine subunits. Strikingly, although lysozymes are ubiquitous in a diversity of organisms, from animals to viruses, they are extremely rare in fungi and have not been described in plants. Recently, we showed that the previously identified virulence factor VdAve1 that is secreted by the soil-borne fungal plant pathogen Verticillium dahliae, is an antibacterial protein that promotes plant colonization through the manipulation of host microbiome compositions. Moreover, transcriptome and forward genetic analyses uncovered a similar involvement of $B$. subtilis genes in protection against $\mathrm{VdAve} 1$ and against lysozyme, suggesting that $\mathrm{VdAve} 1$ may act as a lysozyme. However, VdAve1 shares no homology with known hydrolytic enzymes and is not predicted to carry an enzymatic domain. In this study, we show that $\mathrm{VdAve} 1$ is able to hydrolyze peptidoglycan, hereby uncovering the effector as a novel type of lysozyme. Besides the hydrolytic activity, we show that VdAve1, like many previously described lysozymes, also exerts a nonenzymatic antimicrobial activity that involves direct cell membrane perturbation, which is mediated by an arginine- and lysine-rich peptide sequence of the protein. As $V$. dahliae originally acquired VdAve 1 through horizontal gene transfer from plants, where $\mathrm{VdAve} 1$ homologs are ubiquitous, we speculate that these homologs are the so far enigmatic plant lysozymes. Collectively, VdAve1, as well as its plant homologs, are an untapped resource of novel lysozymes that may be exploited for the development of novel antimicrobials.
\end{abstract}




\section{Introduction}

The bacterial cell envelope is a dynamic multilayered structure that forms the barrier between a bacterium and its environment ${ }^{178}$. Generally, bacteria are classified based on their cell wall architecture either as Gram-positive or Gram-negative. The cell wall of Gram-positive bacteria consists of a thick peptidoglycan layer and a single cell membrane. Consequently, Gram-positive bacteria are also commonly referred to as so-called monoderms. Peptidoglycan is a macromolecule composed of glycan chains consisting of alternating $\mathrm{N}$-acetylmuramic acid (MurNAc) and $\mathrm{N}$-acetylglucosamine (GlucNAc) residues that are linked through $\beta-1,4$ glycosidic bonds ${ }^{179,180}$. These glycan chains are crosslinked by peptides between $N$-acetylmuramic acid residues, which results in an overall rigid mesh-like structure. Additionally, the cell envelopes of most Gram-positives contain polyanionic glycopolymers, the teichoic acids, which comprise membrane bound lipoteichoic acids and peptidoglycan bound wall teichoic acids ${ }^{151,162}$. The cell wall of Gram-negatives, or diderms, contains a much thinner layer of peptidoglycan that is located between the plasma- and the outer membrane, the so-called periplasm. The outer membrane is covered by a thick layer of (glyco) lipids, typically lipopolysaccharides, which forms the barrier between Gram-negative bacteria and their environment.

The bacterial cell envelope is an important target for antimicrobial compounds. Lysozymes represent a wide-spread and well characterized group of antimicrobial enzymes that hydrolyze the $\beta-1,4$ glycosidic bonds between the $N$-acetylmuramic acid and $\mathrm{N}$-acetylglucosamine subunits of the peptidoglycan. Although they are produced by a diversity of higher and lower organisms ${ }^{181-183}$, lysozymes have predominantly been identified in animals where they fulfill important roles in innate immune responses ${ }^{184}$. Animal lysozymes are broadly categorized into three types: c-type (chicken type), g-type (goose-type) and i-type (invertebrate type). The c-type is the most wellcharacterized type that, amongst others, comprises the well-studied hen egg white (HEWL) and human lysozyme. Although lysozymes from different types share little to no sequence homology, some structural characteristics are shared by many lysozymes, such as a deep cleft containing the active site of the proteins ${ }^{184}$. Furthermore, several sequence-unrelated antimicrobial peptides have been identified in lysozymes of different classes ${ }^{147,148,185}$. For instance, the bacteriophage T4 (T4L) and human c-type lysozyme carry an arginine-rich cationic antimicrobial peptide that acts independently from the hydrolytic activity, as was demonstrated by bacterial cell killing through the use of catalytically inactivate mutant proteins as well as through lysozyme-derived peptides ${ }^{147-149,186}$. These findings indicate that lysozymes can target bacteria through two distinct mechanisms and not exclusively through peptidoglycan hydrolysis.

Many bacteria use extracytoplasmic function (ECF) sigma factors to induce transcriptional changes in response to environmental stimuli, including cell envelope 
active antimicrobial compounds ${ }^{187}$. The Gram-positive bacterium Bacillus subtilis encodes seven ECF sigma factors, several of which are involved in protection against antimicrobials $^{144}$. The $B$. subtilis ECF sigma factor $V($ SigV) plays a central role in protection against (hen egg white) lysozyme ${ }^{145,146}$. SigV-mediated protection of $B$. subtilis against lysozyme relies on the induction of two operons, the sig $V$ and the $d l t A B C D E$ operon ${ }^{144,146}$. The sigV operon comprises four genes that, besides the genes encoding SigV and its anti-sigma factor RsiV, also includes yrhK and oatA. Whereas the function of $y$ rhK remains elusive, oat $A$ encodes an acetyltransferase that mediates O-acetylation of muramic acid residues in peptidoglycan, a modification that reduces the sensitivity towards the hydrolytic activity of lysozyme ${ }^{146,147,150}$. Additionally, the SigV-mediated induction of the dltABCDE operon facilitates D-alanylation of teichoic acids, which increases the overall charge of these cell wall components and results in enhanced tolerance to lysozyme, presumably through repelling the positively charged protein $^{146,147}$.

To promote disease establishment on their hosts, pathogenic microbes, including fungal plant pathogens, secrete a plethora of so-called effector molecules that are typically thought to modulate host immunity or physiology 1,25,131. Like all higher organisms, plants associate with numerous microbes that collectively make up their microbiota, which fulfills a prominent role for their well-being. Plants shape the composition of their microbiomes, i.e. the microbes and their genomes in their environment ${ }^{6}$, through the secretion of specific root exudates that promote the recruitment of beneficial microbes aiding in the protection against various stresses including pathogens $9,11,15-18,21,85,188$. Consequently, we recently hypothesized that plant pathogenic microbes exploit effector molecules to impact beneficial plantassociated microbial communities to facilitate host colonization ${ }^{78}$. Accordingly, we recently showed that the previously identified virulence factor $\mathrm{VdAve} 1$ that is secreted by the soil-borne fungal pathogen Verticillium dahliae during host colonization ${ }^{61}$, is an antibacterial protein that promotes disease establishment through manipulation of host microbiome compositions ${ }^{134}$. More specifically, we showed that secretion of VdAve1 by $V$. dahliae suppresses proliferation of antagonistic Sphingomonads in planta, and consequently facilitates host colonization by the fungus ${ }^{134}$. Importantly, however, in vitro activity assays on a panel of plant-associated bacteria revealed that the activity spectrum of VdAve1 is not restricted to Sphingomonads. Interestingly, whereas VdAve1 differentially affects Gram-negative bacteria, all Gram-positive bacteria tested thus far, are severely affected by the effector protein, including the Gram-positive model organism B. subtilis that is typically found in soil, and in the gastrointestinal tract of ruminants as well as of humans ${ }^{134}$. Characterization of the VdAve1 activity on $B$. subtilis using various microscopic approaches revealed that the effector rapidly induces cell lysis and that its activity does not require cell division, suggesting it directly acts on the bacterial cell envelope (Chapter 4$)^{134}$. 
Intriguingly, VdAve1 is homologous to the widespread family of plant natriuretic peptides (PNPs) that are ubiquitous among plant species, and phylogenetic analysis places VdAve1 among its plant homologs, which suggests that $V$. dahliae acquired VdAve1 through horizontal gene transfer from plants ${ }^{61}$. PNPs have predominantly been implicated in the regulation of plant homeostasis and in (a)biotic stress responses $79,80,135$. However, we recently showed that AtPNP-A from the model plant Arabidopsis thaliana, like VdAve1, effectively inhibits $B$. subtilis growth ${ }^{134}$. Hence, the antimicrobial activity might be an ancestral activity in plants that is a common trait among PNPs.

Like many cell envelope-active antimicrobial proteins (AMPs), VdAve1 is relatively small $(\sim 12 \mathrm{kDa})$ and positively charged $(\mathrm{pl} \sim 9)$. Many cationic AMPs establish electrostatic interactions with anionic components of the bacterial cell envelope to exert their activity ${ }^{110}$. Typically, such activities are associated with salt and mineral sensitivity, as cations such as $\mathrm{Na}^{+}, \mathrm{Mg}^{2+}$ and $\mathrm{Ca}^{2+}$ impede access to anionic target molecules. Accordingly, similar cation sensitivity was detected for the activity of VdAve1 (Chapter 4). Although the mode of action underlying the lytic activity of $\mathrm{VdAve} 1$ remains elusive, recent transcriptome and forward genetic analyses performed on $B$. subtilis revealed a central role for the sigV regulon in response to the effector protein (Chapter 4). In line with previous observations following treatment with lysozyme, but not with other cell envelope active compounds, we observed that treatment with $\mathrm{VdAve} 1$ induces the expression of the $\operatorname{sig} V$ and $d / t A B C D E$ operons in $B$. subtilis. Moreover, mutagenesis of several SigV-regulated genes, including oat $A$ and $d / t A-E$, increased sensitivity to $\mathrm{VdAve1}$. These findings suggest that the peptidoglycan and teichoic acid modifications mediated by oat $A$ and the dltABCDE operon protect against VdAve1 in a similar fashion as against lysozyme. Collectively, the striking similarity between the transcriptional changes induced by VdAve1 and lysozyme, combined with the demonstrated involvement of SigV-regulated genes in protection against both proteins, points towards lysozyme-like activity of VdAve1. Here, we address the hypothesis that $\mathrm{VdAve} 1$ acts as a lysozyme in more detail by assessing its potential to hydrolyze peptidoglycan as well as its ability to act as a non-enzymatic cationic AMP.

\section{Results}

Many cationic AMPs exert their antimicrobial activity through the formation of pores in cell membranes ${ }^{110}$, which leads to a rapid loss of membrane potential. To gain more insight in the $\mathrm{VdAve} 1$ killing kinetics and the time required to induce $B$. subtilis cell lysis, we incubated the bacteria with the fluorescence potentiometric probe $\operatorname{DiSC}_{3}(5)^{189}$ and monitored dissipation of the membrane potential by quantifying the increase in fluorescence. As a positive control, a mix of the ion channel-like pore forming peptides gramicidin $A, B$ and $C$ was used ${ }^{190}$, which caused immediate membrane depolarization 
in a fashion that is typically observed for pore-forming compounds (Fig. 1). Like gramicidin, also VdAve1 induced depolarization of the bacterial cells, a phenomenon that occurs in a concentration-dependent manner. However, when compared with gramicidin, the depolarization occurs considerably more gradually, which suggests that it is not based on immediate membrane disruption by pore formation (Fig. 1). Collectively, these observations reinforce our previous findings that $\mathrm{VdAve} 1$ induces bacterial cell membrane disruption, but suggest that this disruption is not based on a typical cationic AMP-like activity that involves pore-formation. Rather, gradual lysis of the bacterial cells seems to take place, a phenomenon that is observed upon hydrolysis of cell wall peptidoglycan, for example.

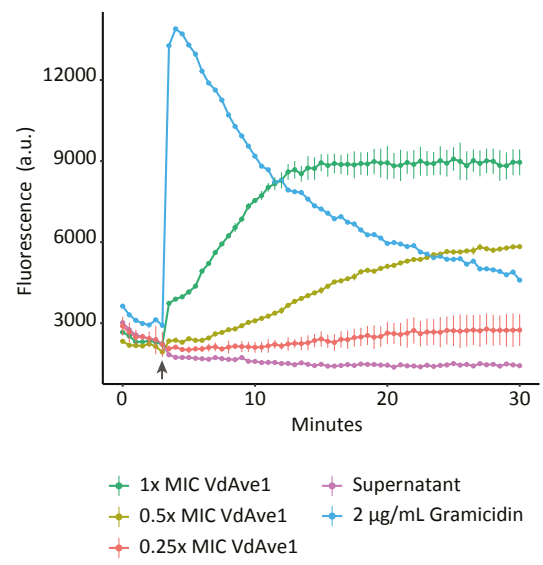

FIGURE 1 | VdAve1 induces gradual depolarization of $B$. subtilis cell membranes. Depolarization of B. subtilis 168 WT cell membranes was determined by quantification of release of the fluorescent dye $\mathrm{DiSC}_{3}(5)$ by the bacterial cells upon incubation with various VdAve 1 concentrations in arbitrary units (a.u.). The supernatant obtained following affinity precipitation of $\mathrm{VdAve} 1$ was included as a negative control. $\mathrm{A}$ mix of the pore-forming peptides gramicidin $A, B$ and $C$ was used as a positive control. The arrow indicates the time point of protein administration. Graphs display the average fluorescence of three biological replicates \pm SD.

To determine if VdAve1 indeed exerts hydrolytic activity, we mixed peptidoglycan purified from $B$. subtilis cell walls with the effector protein and determined the turbidity of the suspension following 15 minutes of incubation at room temperature. Additionally, we also included the homologous protein VnAve1 from the nonpathogenic sister species $V$. nubilum in this assay. VnAve1 displays $90 \%$ amino acid identity with VdAve1 and was previously shown to induce similar transcriptomic changes in B. subtilis (Chapter 4). Strikingly, incubation of both effector proteins with $B$. subtilis peptidoglycan resulted in a strongly reduced turbidity, indicating that both proteins hydrolyze peptidoglycan (Fig. 2a).

Antimicrobial activity assays revealed strong activity of VdAve1 on all Gram-positive bacteria tested thus far, namely B. subtilis, Arthrobacter sp., Streptomyces sp. and 
Staphylococcus xylosus ${ }^{134}$. Interestingly, Staphylococci have been reported to display differential sensitivity to lysozymes ${ }^{150,191}$. Whereas pathogenic species including Staphylococcus aureus are insensitive for the hydrolytic activity of many lysozymes, nonpathogenic species, such as S. xylosus, are generally very sensitive ${ }^{150,191}$. Consequently, we tested if $S$. aureus peptidoglycan is sensitive for hydrolysis by VdAve1. Interestingly, like for HEWL, no hydrolysis could be detected following incubation of $S$. aureus peptidoglycan with VdAve1 or VnAve1 (Fig. 2b). Additionally, to confirm that S. aureus cells are also insensitive for VdAve1, we monitored in vitro growth of the bacterium in the presence of $16 \mu \mathrm{M}$ of the effector protein, corresponding to $2 \times \mathrm{MIC}$ for $B$. subtilis. Indeed, no growth inhibition was observed (Fig. 2c), suggesting that the peptidoglycan hydrolase activity of $\mathrm{VdAve} 1$ is instrumental for its antimicrobial activity. Moreover, the corresponding differential activities of VdAve1 and HEWL as observed on the peptidoglycan of $B$. subtilis and $S$. aureus suggest that the proteins act through similar mechanisms, and indicate that $\mathrm{VdAve} 1$ might act as a lysozyme that hydrolyzes the $\beta-1,4$ glycosidic bonds of the glycan chain.

a

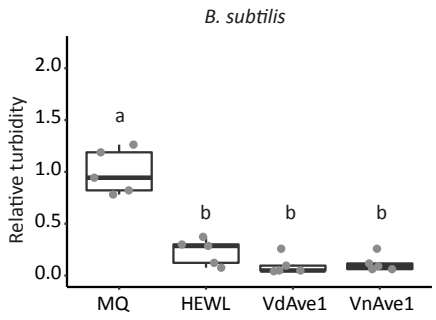

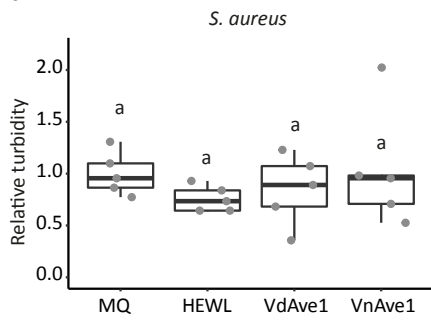

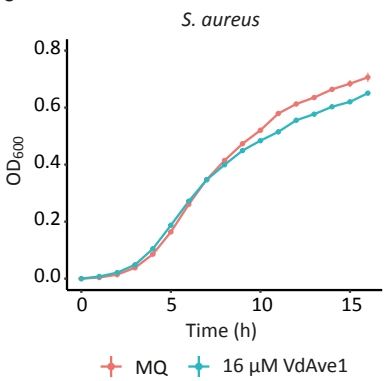

FIGURE 2 | VdAve1 and VnAve1 selectively hydrolyze peptidoglycan of Gram-positive bacteria. VdAve1 and VnAve1 hydrolyze peptidoglycan purified from B. subtilis (a) but not from S. aureus (b) cell walls as revealed by reduced turbidity of the suspensions. Hen egg white lysozyme (HEWL) and MQ were included as positive and negative controls, respectively. Different letter labels represent statistically significant differences according to one-way ANOVA and Tukey's post-hoc test with N=5, p<0.01 (a) or $\mathrm{p}<0.05$ (b). (c) VdAve1 does not affect $S$. aureus growth in vitro. Graphs display the average $O D_{600}$ of three biological replicates \pm SD.

To corroborate the hypothesis that VdAve1 targets the $\beta-1,4$ glycosidic bonds of the glycan chain and, for instance, does not act as a protease that hydrolyses peptidoglycan crosslinks, we monitored the hydrolysis of $B$. subtilis peptidoglycan by $\operatorname{VdAve} 1$ in the presence of protease inhibitors and excess peptidoglycan pentapeptide, a precursor of the crosslinks in peptidoglycan. Neither of the treatments impaired the hydrolase activity of VdAve1 (Fig. 3a), which reinforces the suggestion that degradation of peptidoglycan by $\mathrm{VdAve} 1$ is based on hydrolysis of the $\beta-1,4$ glycosidic bonds of the glycan chains. 
Recent antimicrobial activity assays on $B$. subtilis revealed strong inhibition of VdAve1 activity by lipoteichoic acids and cationic molecules such as sodium ions (Chapter 4). To test if the hydrolytic activity of $\mathrm{VdAve} 1$ is also affected by such compounds, we quantified hydrolysis of $B$. subtilis peptidoglycan by $\mathrm{VdAve} 1$ in the presence of lipoteichoic acids and increasing sodium chloride concentrations. Indeed, supplementation of the assay solution with lipoteichoic acids almost completely abolished peptidoglycan hydrolysis by VdAve1 (Fig. 3b). Similarly, sodium chloride concentrations as low as $6.25 \mathrm{mM}$ significantly affected the hydrolytic activity of the effector protein (Fig. 3c). Thus, compounds that were previously shown to inhibit the antimicrobial activity of VdAve1 on $B$. subtilis cells, indeed affect its peptidoglycan hydrolase activity.

Besides their activity on peptidoglycan, many lysozymes, including HEWL, are also capable to hydrolyze the glycosidic bonds of the $N$-acetyl-D-glucosamine homopolymer chitin, a major constituent of fungal cell walls. Hence, some lysozymes exert antibacterial as well as antifungal activities. However, in vitro growth assays of various fungal species in the presence of $\mathrm{VdAve} 1$ revealed no antifungal activity of the effector protein, suggesting inability to hydrolyze fungal cell walls ${ }^{134}$. To confirm that VdAve1 does not exert chitinase activity, we incubated the protein with the endochitinase substrate 4-Methylumbelliferyl $\beta-D-N, N^{\prime}, N^{\prime \prime}$-triacetylchitotriose, and quantified release of the fluorophore 4-Methylumbelliferyl from the substrate as a proxy for endochitinase activity, while HEWL was used as a positive control. As anticipated, no endochitinase activity was detected for VdAve1 or for VnAve1, while HEWL displayed clear endochitinase activity (Fig. 3d).

In addition to their hydrolytic activities, several lysozymes also affect microbes through non-enzymatic activities that involve the perturbation of cell membranes ${ }^{147-149,186}$. Interestingly, the membrane disruptive activities of T4L and HEWL are not restricted to prokaryotic cell membranes, as both proteins were previously shown to lyse protoplasts obtained from the mesophyll of potato plants ${ }^{148}$. In contrast, incubation of VdAve1 with protoplasts obtained from cucumber, as one of its host plants, did not induce lysis ${ }^{134}$. However, given that VdAve1 is presumed to be horizontally acquired from plants, and that plant homologs similarly possess antimicrobial activity ${ }^{61,134}$, it is conceivable that plant cells are insensitive to VdAve1 activity. Therefore, to test if VdAve1 is able to disrupt the membranes of other types of organisms, we generated protoplasts of various microbes that do not contain peptidoglycan in their cell walls and exposed these to the effector protein. Strikingly, VdAve1 caused lysis of the protoplasts from the fungal species Trichoderma viride and Monilinia fructicola, that contain chitin as main cell wall constituent, as well as from the oomycete species Phytophthora capsici that, like plants, contains cellulose in its cell walls (Fig. 4a). Collectively, these findings indicate that VdAve1 can perturb cell membranes, and suggest that its bacteriolytic activity might not exclusively depend on cell wall degradation by peptidoglycan hydrolysis. 
a

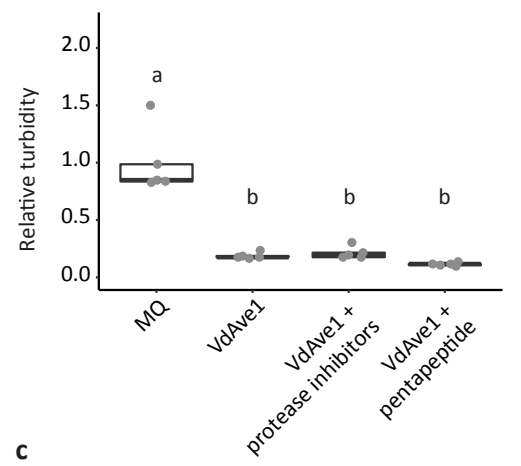

b

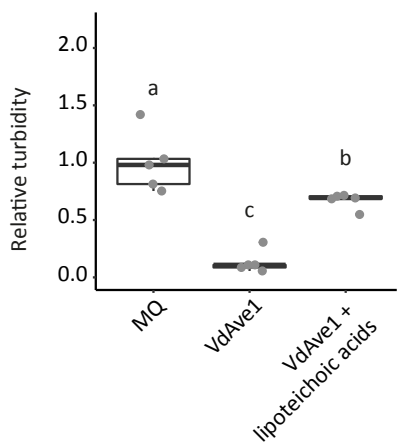

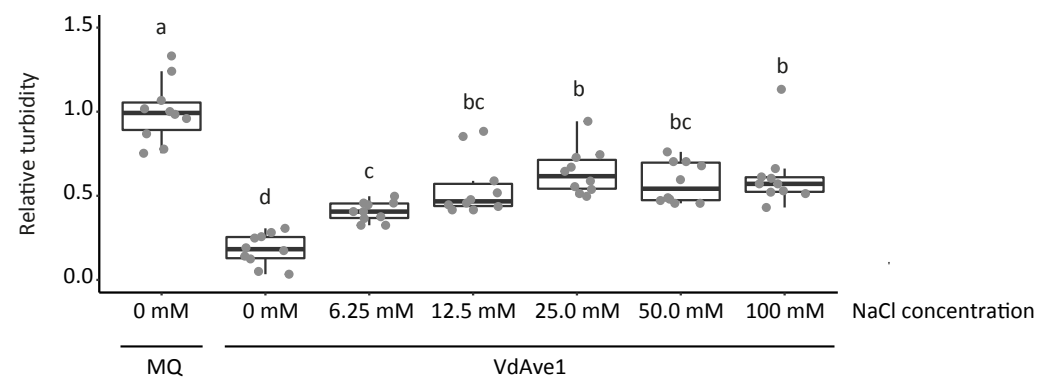

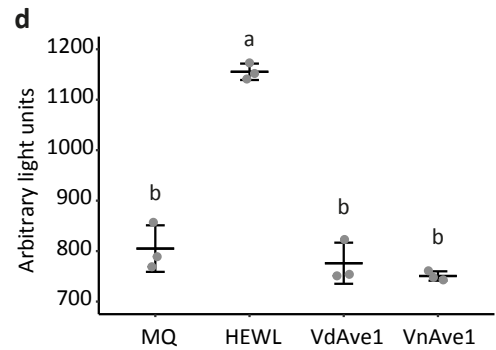

FIGURE 3 | Hydrolytic activity of VdAve1 is not based on protease or endochitinase activity and is inhibited by lipoteichoic acids and sodium chloride. (a) Hydrolysis of $B$. subtilis peptidoglycan by VdAve1 is not impaired by protease inhibitors or peptidoglycan pentapeptide, a precursor of the peptidoglycan crosslinks. Different letter labels represent statistically significant differences according to one-way ANOVA and Tukey's post-hoc test; $p<0.0001, N=5$. (b) Lipoteichoic acids purified from $B$. subtilis cell walls inhibit peptidoglycan hydrolysis by VdAve1. Different letter labels represent statistically significant differences according to one-way ANOVA and Tukey's post-hoc test; $p<0.05, N=5$. (c) The impact of sodium ions on the hydrolytic activity of $\mathrm{VdAve} 1$ as determined by the ability of the effector protein to hydrolyze $B$. subtilis peptidoglycan in the presence of increasing $\mathrm{NaCl}$ concentrations. Letters represent statistically significant differences according to one-way ANOVA and Tukey's post-hoc test; $p<0.05, N=10$. (d) Hydrolytic activities of VdAve1 and VnAve1 share no functional overlap with endochitinases as determined by the inability of the effector proteins to release the fluorophore 4-Methylumbelliferyl from the endochitinase substrate 4-Methylumbelliferyl $\beta-D-N, N^{\prime}, N^{\prime \prime}$-triacetylchitotriose. Hen egg white lysozyme (HEWL) and $M Q$ were included as positive and negative controls, respectively. Different letter labels represent statistically significant differences according to one-way ANOVA and Tukey's post-hoc test; $p<0.0001, N=3$. The plot displays the average arbitrary light units \pm SD. 
a T. viride M. fructicola P. capsici
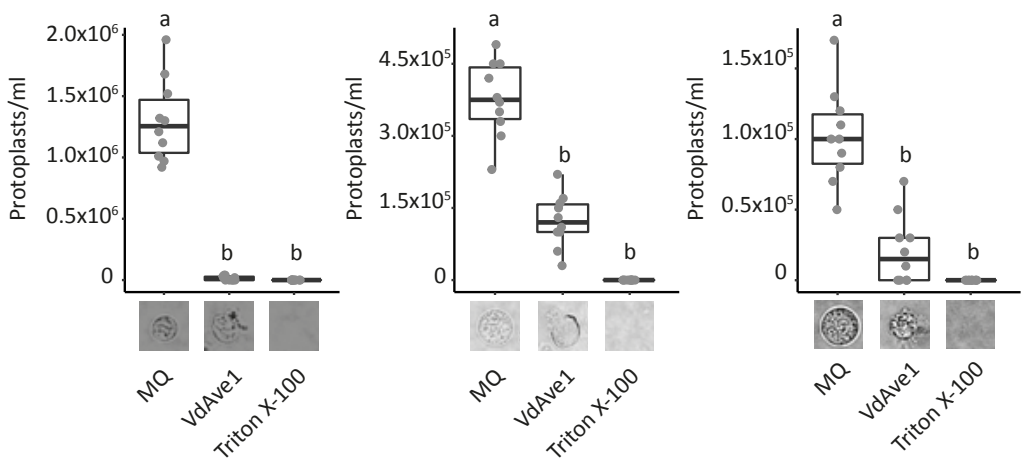

b

B. subtilis

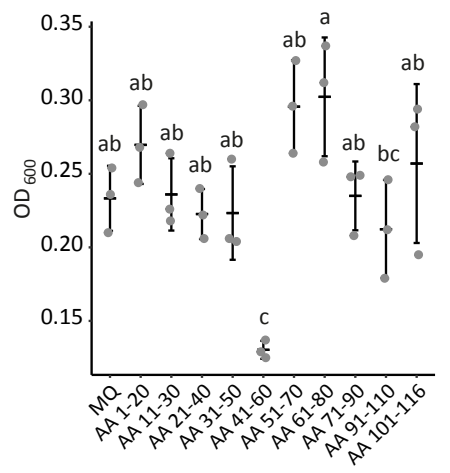

Peptide Sequence

AA 1-20 DLGTASYYNPPYLPTACGGS

AA 11-30 PYLPTACGGSNPSOFPSGNL

AA 21-40 NPSQFPSGNLFVAVSDGLWD

AA 31-50 FVAVSDGLWDNGAACGRRYR

AA 41-60 NGAACGRRYRIKCLSGARGS

AA 51-70 IKCLSGARGSCKDGMIDVRV

AA 61-80 CKDGMIDVRVVDRAKTTVTK

AA 71-90 VDRAKTTVTKAAHKATMILS

AA 91-110 QDSYDAIVNQWKGTRHKAVN

AA 101-116 WKGTRHKAVNIEFRQI

c

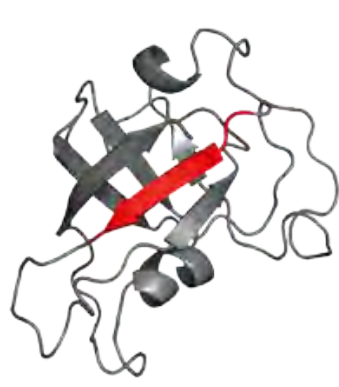

FIGURE 4 | Protoplast lysis and identification of a VdAve1-derived antimicrobial cationic peptide suggest membrane lytic activity. (a) VdAve1 disrupts fungal and oomycete protoplasts. Protoplasts were obtained from the fungi Trichoderma viride, Monilinia fructicola and the oomycete Phytophthora capsici and incubated with $8 \mu \mathrm{MVdAve} 1$. After 30 minutes, the number of protoplasts was quantified using a haemocytometer. Triton $\mathrm{X}-100$ and $M Q$ were included as positive and negative control for protoplast disruption, respectively. Representative phenotypes of the protoplasts under the various experimental conditions are displayed under the boxplots. Different letter labels represent statistically significant differences according to one-way ANOVA and Tukey's post-hoc test; $\mathrm{p}<0.0001, \mathrm{~N}=10$. (b) A single cationic VdAve1-derived peptide displays antimicrobial activity. Optical density of $B$. subtilis cultures following seven hours of in vitro cultivation in low-salt LB supplemented with $50 \mu \mathrm{M}$ of VdAve1-derived peptides generated by in vitro synthesis. Only the peptide encompassing AA 41-60 of VdAve1, comprising the arginineand lysine-rich sequence "RRYRIK" (red font) inhibits B. subtilis growth. Different letter labels represent statistically significant differences according to one-way ANOVA and Tukey's post-hoc test; $p<0.05, N=3$. The plot displays the average $\mathrm{OD}_{600} \pm \mathrm{SD}$. (c) The cationic VdAve1-derived peptide is predicted to form a surface-exposed $\beta$-sheet of the protein. The arginine- and lysine-rich sequence "RRYRIK" in the structural model of VdAve 1 is highlighted in red. Structural model was obtained using Phyre2 with a confidence score of $100 \%{ }^{103}$.

Non-enzymatic membrane lytic activities of lysozymes typically depend on cationic arginine-rich regions of the proteins ${ }^{147-149,186}$. Like lysozymes, also VdAve1 is a positively charged protein that comprises multiple arginine- and/or lysine-rich 
regions. To identify potential regions of $\mathrm{VdAve} 1$ that mediate membrane disruption, we monitored in vitro growth of $B$. subtilis in the presence of individual 20 AA VdAve1derived partially overlapping peptides, that collectively span the complete protein sequence. Strikingly, a single peptide (AA 41-60) comprising the arginine- and lysinerich sequence "RRYRIK" inhibited $B$. subtilis growth, while all the other peptides, including two partially overlapping peptides that split the arginine- and lysine-rich sequence in two halves (AA 31-50 and AA 51-70), did not display antimicrobial activity (Fig. 4b). Intriguingly, structural analysis of a high confidence protein model obtained for VdAve1 (Phyre ${ }^{2}$, confidence score $\left.100 \%\right)^{103}$, suggests that the arginine- and lysinerich sequence forms a surface-exposed $\beta$-sheet of the effector (Fig. 4c), which supports the potential involvement of these amino acids in membrane disruption.

\section{Discussion}

Lysozymes represent a widely distributed group of functionally analogous enzymes predominantly identified in animals where they act as crucial components of innate immune systems ${ }^{184}$. Recently, we described that the previously identified secreted $V$. dahliae virulence factor $\mathrm{VdAve} 1$ is an antimicrobial protein that promotes plant colonization by manipulation of host microbiome compositions ${ }^{134}$. Moreover, we demonstrated that $V$. dahliae utilizes $V d A v e 1$ to target microbial antagonists ${ }^{134}$. Recent transcriptome and forward genetic analyses revealed a striking similarity of $B$. subtilis genes involved in protection against $\mathrm{VdAve} 1$ and against lysozyme, involving a central role for the sigV regulon (Chapter 4), suggesting that $\mathrm{VdAve} 1$ possesses lysozyme activity. In this chapter we provide functional data to uncover VdAve1 as a novel type of lysozyme. Reports of fungal peptidoglycan hydrolases are extremely rare, as the only fungal peptidoglycan hydrolase described thus far is the lysozyme from Chalaropsis sp. ${ }^{183,192}$. However, VdAve1 does not display any degree of sequence similarity to this Chalaropsis sp. lysozyme.

Incubation of VdAve1 and VnAve1 with peptidoglycan purified from B. subtilis revealed that both effector proteins exert peptidoglycan hydrolase activity. Intriguingly, neither VdAve1, nor any of its homologs, carry previously described enzymatic domains or share homology with known hydrolytic enzymes. Consequently, following its identification in V. dahliae ${ }^{61}$, and even after its characterization as an antimicrobial protein ${ }^{134}, \mathrm{VdAve} 1$ was not anticipated to act as a hydrolytic enzyme. The lack of homology with previously characterized hydrolytic enzymes indicates that VdAve1 and VnAve1 constitute a completely novel class of lysozymes.

To protect themselves against pathogenic invaders, plants secrete a diversity of antimicrobials including hydrolytic enzymes ${ }^{193}$. Such hydrolases comprise, amongst others, chitinases and glucanases that aid in the suppression of eukaryotic microbial pathogens ${ }^{193-195}$. Strikingly, however, lysozyme-like hydrolytic enzymes intended for 
suppression of bacterial pathogens have not been described in the plant kingdom. Intriguingly, we previously demonstrated that $V$. dahliae acquired VdAve1 through horizontal gene transfer from plants where VdAve1 homologs, so called PNPs, are ubiquitous. Importantly, we recently observed that the closest VdAve1 homolog of A. thaliana, AtPNP-A, also displays strong antimicrobial activity on B. subtilis ${ }^{134}$. The identified peptidoglycan hydrolase activity of $\mathrm{VdAve1}$, combined with the conserved antimicrobial activity as observed for AtPNP-A, therefore strongly suggests that AtPNP-A acts as a plant lysozyme. Moreover, these observations suggest that PNPs represent a wide-spread family of plant lysozymes that have remained undescribed even long after the identification of lysozymes in many other organisms. Along these lines it is interesting to note that AtPNP-A has previously been proposed to be involved in plant immune responses ${ }^{79,196}$. Transcriptome analyses on A. thaliana revealed that AtPNP-A is coregulated with genes encoding so-called pathogenesis-related $(P R)$ proteins that include the above-mentioned chitinases and glucanases ${ }^{193,194,196}$, and that are involved in immune responses against biotic stressors ${ }^{196}$. Chitinases degrade fungal cell walls through the hydrolysis of the glycosidic bonds of the $N$-acetyl-D-glucosamine homopolymer chitin, a major constituent of fungal cell wall. Interestingly, many plant chitinases are bifunctional enzymes that also display some degree of peptidoglycan hydrolase activity ${ }^{197-199}$. It is important to note, however, that this activity is generally much weaker than their chitinase activity. Consequently, it is tempting to speculate that PNPs act as genuine lysozymes and play a much more prominent role in the protection against bacterial pathogens.

The precise target within the peptidoglycan molecule of the hydrolase activity of $\mathrm{VdAve} 1$ remains presently unknown. The highly similar defense mechanisms induced by $B$. subtilis in response to VdAve1 and lysozyme (Chapter 4), and the observation that the VdAve1mediated hydrolysis is not based on protease activity, strongly suggests that VdAve1 targets the $\beta-1,4$ glycosidic bonds of the glycan chains. Comparative analysis of HPLC elution profiles following treatment of peptidoglycan with VdAve 1 and with lysozyme, or mass spectrometry-based analyses of $\mathrm{VdAve} 1$-hydrolysed peptidoglycan fragments, can be used to reveal the exact target of the hydrolytic activity.

Besides the target, also the catalytic residues that mediate peptidoglycan hydrolysis remain enigmatic. Substrate hydrolysis by enzymes like chitinases and lysozymes is mediated by the negatively charged residues of aspartic and glutamic acids. VdAve1 contains eight aspartic acids and a single glutamic acid, some of which are strongly conserved among VdAve1 homologs, while others are unique for VdAve1 and VnAve1. Site-directed mutagenesis of these amino acids should uncover the catalytic residues responsible for the peptidoglycan hydrolase activity of $\mathrm{VdAve} 1$ and could simultaneously provide an indication for the conservation of this activity across the plant homologs. Moreover, successful mutagenesis of the catalytic residues could facilitate the characterization of the non-enzymatic antimicrobial activity of $\mathrm{VdAve} 1$ and might help to uncouple the contribution of the different activities to the inhibition of different bacterial isolates. 
Although the characterization of $\mathrm{VdAve} 1$ as a peptidoglycan hydrolase can explain the strong sensitivity of Gram-positive bacteria for the effector protein, it does not explain the previously described antimicrobial activity on Gram-negatives like Sphingomonads ${ }^{134}$. Likely, the activity of VdAve1 on Gram-negative bacteria is determined by its non-enzymatic membrane lytic activity that was uncovered following incubation of the effector protein with fungal and oomycete protoplasts. Intriguingly, the ability of VdAve1 to disrupt fungal protoplasts is in contrast to our previous observations that incubation of various fungal species with $\mathrm{VdAve} 1$ did not affect their growth ${ }^{134}$. Possibly, the fungal cell wall functions as a protective barrier that inhibits the $\mathrm{VdAve} 1$ activity and that perturbs access of the protein to fungal cell membranes. In this respect is interesting to note that, in contrast to many lysozymes ${ }^{200,201}$, VdAve1 does not exert endochitinase activity, suggesting that the chitin in fungal cell walls does not serve as a substrate for the hydrolytic activity of the effector. Nevertheless, chitin may still be bound by the active site of $\mathrm{VdAve} 1$ and in this manner it might block access to the cell membranes. Various $N$-acetyl-D-glucosamine (GlcNAc) oligomers, subunits of the fungal cell well polymer chitin, have previously been described to bind the active site of lysozymes and to therefore inhibit peptidoglycan hydrolysis ${ }^{202-204}$. The chitin in fungal cell walls might similarly inhibit VdAve1.

Like for previously characterized lysozymes ${ }^{147-149,186}$, the non-enzymatic membrane lytic activity of VdAve1 seems to depend on an arginine- and lysine-rich sequence of the protein, namely "RRYRIK". Intriguingly, this short sequence partially overlaps the highly conserved PNP domain that is found in nearly all VdAve1 homologs 61,205,206, and which is typically flanked by positively charged amino acids residues. Accordingly, the closest VdAve1 homologs in A. thaliana (AtPNP-A) and tomato (SIAve1) carry the homologous amino acid sequences "RRYRVR" and "RRYRLR", respectively. Hence, arginine- and lysine-rich sequences homologous to the one identified in VdAve1 can be anticipated to contribute to the antimicrobial activities of PNPs like AtPNP-A.

The dual functionality of VdAve1, involving a peptidoglycan hydrolase activity combined with a non-enzymatic membrane disruptive activity, shows strong similarity with the antimicrobial activity of other lysozymes. Arguably, these two activities complement each other as the peptidoglycan hydrolase activity of VdAve1 is likely to facilitate the access of the cationic peptide to the bacterial cell membrane of monoderms. Conversely, on diderms the cationic peptide might first perturb the outer cell membrane in order to expose the peptidoglycan in the periplasm. Dual functionalities have been observed in lysozymes belonging to different classes including c-type, $\mathrm{g}$-type and phage lysozymes, none of which share sequence homology with VdAve1 147-149,185,186. Interestingly, although they are not sequence related, based on their conserved secondary and tertiary structures, HEWL, goose egg white lysozyme (GEWL) and T4L are believed to have evolved from a common ancestor 207,208 . Intriguingly, whereas the secondary structures of these lysozymes predominantly comprise alpha helices ${ }^{207,208}$, 
the predicted structure of $\mathrm{VdAve} 1$ suggests it primarily consists of beta sheets and only contains two small alpha helices (Fig. 4c). Hence, it seems plausible that the similar antimicrobial activities as exerted by VdAve1 when compared with HEWL, GEWL and $\mathrm{T} 4 \mathrm{~L}$ are the result of convergent evolution, which underlines the importance of the dual functionalities as typically observed in lysozymes.

In conclusion, we here described the characterization of the effector protein VdAve1 as a novel type of fungal lysozyme secreted by the plant pathogen V. dahliae. Importantly, our findings might lead to the identification of a presently undescribed family of widely distributed plant lysozymes. Future research will have to reveal whether the VdAve1 homologs in plants, belonging to the large family of PNPs, similarly possess lysozyme activity. The potential wealth of these novel lysozymes may be exploited for the development of novel types of antimicrobials that can be applied in food preservation or for medical or veterinary purposes.

\section{Materials and methods}

Production and purification of recombinant effector proteins. Recombinant VdAve1 and VnAve1 were produced and purified as described previously ${ }^{134}$. However, to avoid the potential co-purification of trace amounts of lysozyme with the effector proteins, E. coli BL21 cells were lysed using 6M guanidine hydrochloride following protein expression.

Membrane potential measurement. Membrane potential measurements were performed as described previously ${ }^{140,189}$, on $B$. subtilis 168 cultures $\left(\mathrm{OD}_{600}=0.1\right)$ growing at $30^{\circ} \mathrm{C}$ in $0.5 x$ YT supplemented with 8,4 or $2 \mu \mathrm{MVdAve} 1$ (1, 0.5 and $0.25 \mathrm{x}$ $\mathrm{MIC}$ ) or $2 \mu \mathrm{g} / \mathrm{mL}$ Gramicidin.

Peptidoglycan hydrolase assay. To test the peptidoglycan hydrolase activity of VdAve1 and VnAve1, $10 \mu \mathrm{g}$ of peptidoglycan purified from B. subtilis or S. aureus cell walls (Sigma-Aldrich, St. Louis, MO, USA) was incubated in demineralized water with supplemented with $10 \mu \mathrm{g}$ of the effector proteins in a final volume of $100 \mu \mathrm{L}$. Following 15 minutes of incubation at room temperature, the remaining peptidoglycan was pelleted by 1 minute of centrifugation at 10,000 $\mathrm{g}$. Subsequently, the supernatants were removed and the pellets were resuspended in $100 \mu \mathrm{L}$ of demineralized water and transferred to clear 96 well flat bottom polystyrene tissue culture plates. Turbidity of the suspensions was measured at $450 \mathrm{~nm}$ using a CLARIOstar plate reader (BMG LABTECH, Ortenberg, Germany).

To determine the impact of protease inhibitors on the hydrolase activity of $\mathrm{VdAve} 1 \mathrm{a}$ $1 \times$ stock solution of the cOmplete ${ }^{\mathrm{T}}$, Mini, EDTA-free Protease Inhibitor Cocktail (Roche, Mannheim, Germany) was prepared in demineralized water and used as assay solution. To determine the impact of peptidoglycan pentapeptide (Sigma-Aldrich, St. 
Louis, MO, USA) or lipoteichoic acids purified from B. subtilis cell walls (Sigma-Aldrich, St. Louis, MO, USA) on the peptidoglycan hydrolase activity of VdAve1, the assay solution was supplemented with $1 \mathrm{mM}$ of peptidoglycan pentapeptide or $100 \mu \mathrm{g} / \mathrm{mL}$ of lipoteichoic acids.

Fluorometric endochitinase activity assay. Endochitinase activity of $\mathrm{VdAve} 1$ and VnAve1 was tested using 4-Methylumbelliferyl $\beta-D-N, N^{\prime}, N^{\prime \prime}$-triacetylchitotriose from the Fluorimetric Chitinase Assay Kit (Sigma-Aldrich, St. Louis, MO, USA). To this end, the substrate was resuspended in $10 \mathrm{mM}$ BisTris $\mathrm{pH} 6.5$ to a final concentration of 0.5 $\mathrm{mg} / \mathrm{mL}$ and supplemented with $2.5 \mu \mathrm{M}$ VdAve1, VnAve1 or hen egg white lysozyme (Sigma-Aldrich, St. Louis, MO, USA). Following one hour of incubation at room temperature, the reaction was terminated using the stop solution and release of 4-Methylumbelliferyl was measured at an excitation wavelength of $360 \mathrm{~nm}$ and an emission wavelength of $450 \mathrm{~nm}$ using a CLARIOstar ${ }^{\circledR}$ plate reader (BMG LABTECH, Ortenberg, Germany).

In vitro microbial growth assays. S. aureus strain 113 and $B$. subtilis strain 168 were grown on lysogeny broth agar (LBA) at $28^{\circ} \mathrm{C}$. Single colonies were selected and grown overnight in low salt LB (10 g/L Tryptone, $5 \mathrm{~g} / \mathrm{L}$ Yeast Extract, $0.5 \mathrm{~g} / \mathrm{L} \mathrm{NaCl}$ ) at $28^{\circ} \mathrm{C}$ while shaking at $200 \mathrm{rpm}$. Overnight cultures of $S$. aureus and $B$. subtilis were resuspended to $\mathrm{OD}_{600}=0.05$ in low salt $\mathrm{LB}$ supplemented with $16 \mu \mathrm{M}$ VdAve1, or 50 $\mu \mathrm{M}$ VdAve1-derived peptides chemically synthesized by GenScript (Piscataway, NJ, USA), respectively. Bacterial suspensions were aliquoted in clear 96 well flat bottom polystyrene tissue culture plates and bacterial growth was monitored as described previously ${ }^{134}$.

VdAve1 activity assays on microbial protoplasts. T. viride, M. fructicola and P. capsici protoplasts were obtained as described previously 209,210 . The different protoplasts were collected by 1 minute of centrifugation at $100 \times \mathrm{g}$ and carefully resuspended in $1 \mathrm{M}$ sorbitol, $10 \mathrm{mM}$ MOPS $\mathrm{pH} 6.3$, to a final concentration of $10^{5}-10^{6}$ protoplasts $/ \mathrm{mL}$. Following 30 minutes of incubation, intact protoplasts were quantified for the different treatments using a haemocytometer.

\section{Acknowledgements}

The authors thank Laura Vilanova and Weizhen Wang for technical assistance. B.P.H.J.T. acknowledges support from the Research Council Earth and Life Sciences (ALW) of the Netherlands Organization of Scientific Research (NWO) and from the Deutsche Forschungsgemeinschaft (DFG, German Research Foundation) under Germany's Excellence Strategy - EXC 2048/1 - Project ID: 390686111. 



\section{Chapter 6}

A Verticillium dahlijae defensin-like effector protein promotes microsclerotia formation tos and survival affier host decay

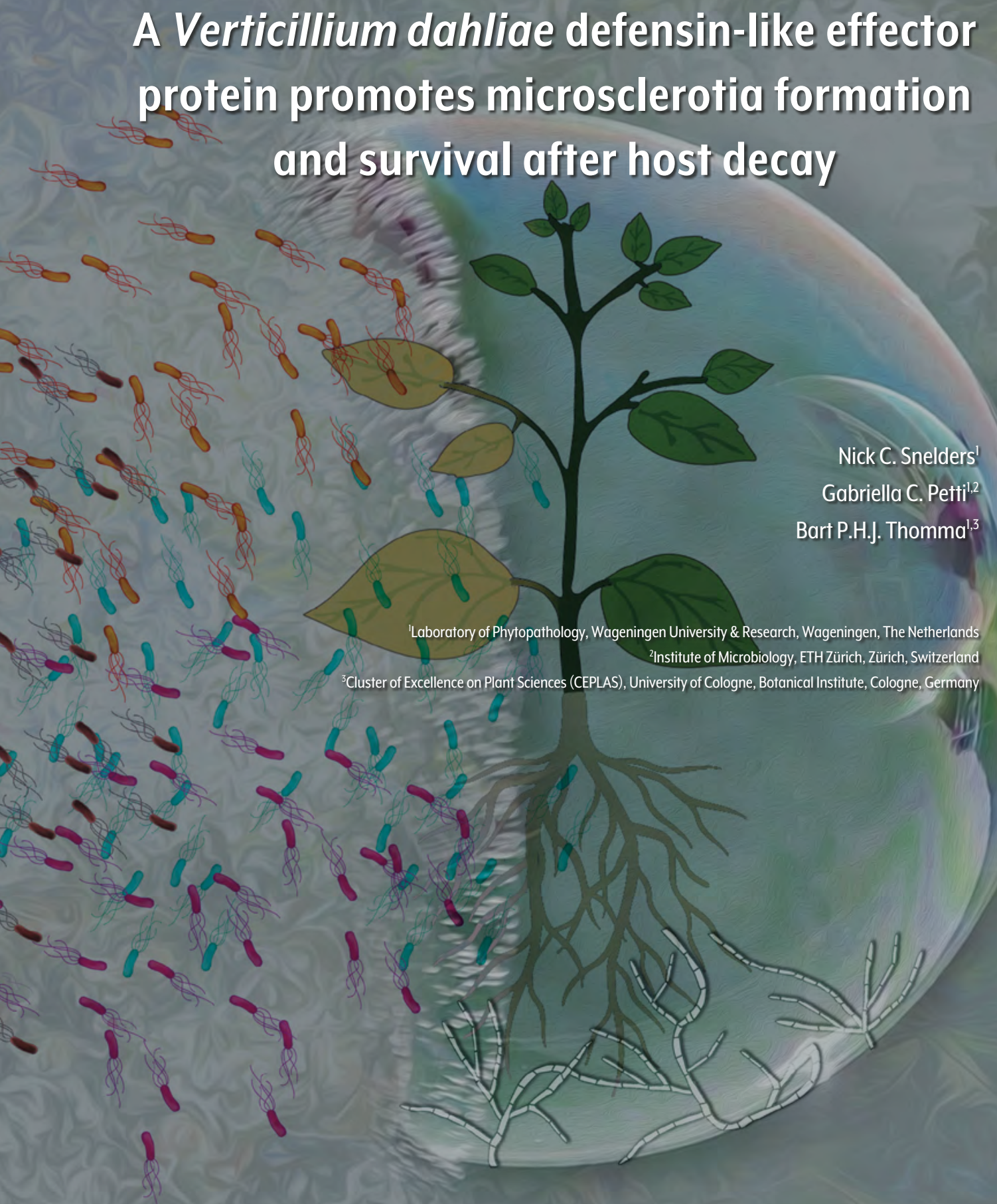




\begin{abstract}
Verticillium dahliae is a soil-borne fungal pathogen that causes vascular wilt disease on hundreds of plant species. After host tissue decomposition, the pathogen can survive in the soil for many years as persistent multicellular melanized resting structures, called microsclerotia, that protect the fungus from (a)biotic stresses. These microsclerotia are formed in the plant tissue at the end of the infection cycle when the plant tissue starts to decay. Like all microbial plant pathogens, V. dahliae secretes effector molecules to facilitate host colonization. We recently reported that $V$. dahliae utilizes part of its effector catalog for microbiome manipulation, inside the host as well as in the soil. Moreover, our data suggest that $V$. dahliae may employ different antimicrobial effector proteins at different life stages. Here we describe the identification and characterization of the defensin-like $V$. dahliae effector protein VdAMP3. We show that VdAMP3 has antimicrobial activity and is expressed during microsclerotia formation. Moreover, based on a reporter strain we reveal that VdAMP3 is specifically expressed in hyphal sections that develop into microsclerotia, suggesting that $V$. dahliae exploits VdAMP3 to protect microsclerotia formation. Accordingly, we show that VdAMP3 contributes to $V$. dahliae biomass accumulation in decaying host tissue. Collectively, our findings demonstrate that $V$. dahliae employs VdAMP3 to protect its microsclerotia and corroborate the hypothesis that $V$. dahliae exploits different antimicrobial effector proteins at different life stages.
\end{abstract}




\section{Introduction}

Verticillium dahliae is a soil-borne asexual fungus that causes vascular wilt disease on hundreds of plant species ${ }^{44}$. The fungus survives in the soil through multicellular melanized resting structures, called microsclerotia, that offer protection against (a)biotic stresses and can persist in the soil for many years ${ }^{211}$. Microsclerotia represent the major inoculum source of $V$. dahliae in nature and their germination is triggered by carbon- and nitrogen-rich exudates from plant roots ${ }^{55}$. Following microsclerotia germination, fungal hyphae grow through the soil and rhizosphere towards the roots of host plants. Next, $V$. dahliae colonizes the root cortex and crosses the endodermis, from which it invades xylem vessels. Once the fungus enters those vessels it forms conidiospores that are transported with the water flow until they get trapped, for instance by vessel end walls. This triggers germination of the conidiospores, penetration of cell walls, followed by hyphal growth and renewed sporulation, leading to systematic colonization of the plant ${ }^{47}$. Once tissue necrosis commences and plant senescence occurs, host immune responses fade and $V$. dahliae enters a saprophytic phase when it emerges from the xylem vessels to invade adjacent host tissues, which is accompanied by the production of microsclerotia. Upon decomposition of plant tissues, these microsclerotia are released in the soil ${ }^{212}$.

Throughout its entire life cycle, $V$. dahliae is exposed to a wealth of potential microbial competitors. The soil in which $V$. dahliae survives during dormant and free-living life stages is extremely rich in microbes and represents a competitive environment where a diversity of organisms compete for space in a limited availability of nutrients, in particular carbon ${ }^{12,213}$. Upon host colonization, $V$. dahliae encounters a plethora of plant-associated microbes that collectively form the plant's microbiota, which represents a key factor for plant health. Beneficial plant-associated microbes are found in and on all organs of the plant and help to mitigate (a)biotic stresses ${ }^{9-11,18,85}$. Consequently, $V$. dahliae successively encounters organ-specific microbiota in the root upon root cortex colonization, in the endosphere during xylem colonization, and in the phyllosphere during tissue necrosis following systemic colonization.

To establish disease, plant pathogenic microbes secrete a plethora of effectors, molecules of various kinds that promote host colonization and that are typically thought to mainly deregulate host immune responses ${ }^{25,214,215}$. However, we previously hypothesized that plant pathogens not only utilize effectors to target components of host immunity as well as other aspects of host physiology to support host colonization, but also to target competitor microbes that are associated with their hosts in order to establish niche colonization ${ }^{25,78}$. Moreover, we recently provided experimental evidence for this hypothesis by showing that the previously identified in planta-induced $V$. dahliae effector VdAve1 is a bactericidal protein that promotes host colonization through the selective manipulation of host microbiomes by suppressing microbial antagonists ${ }^{61,134}$. 
Moreover, when we queried the predicted $V$. dahliae secretome for candidate effectors to act in microbiome manipulation by searching for structural homologs of known antimicrobial proteins (AMPs), we identified ten additional effectors, including a putative Aerolysin-like $\beta$-pore-forming toxin, named VdAMP2. Subsequent functional analysis revealed that VdAMP2 indeed displays antibacterial activity, and that the activity spectrum differs with that of $\mathrm{VdAve} 1^{134}$. Interestingly, $\mathrm{VdAMP} 2$ is not expressed during host colonization, but was found to be induced in the soil where it facilitates niche colonization ${ }^{134}$. Collectively, these observations demonstrate that $V$. dahliae dedicates part of its catalog of effector proteins towards microbiota manipulation during various stages of its life cycle. Thus, it may be anticipated that other effectors can be found that act in microbiome manipulation, and that these display crucial functions during different life- and colonization stages of the pathogen.

One of the previously identified $V$. dahliae effector genes with a putative antimicrobial fold, here named $V d A M P 3$, is predicted to encode a cysteine-stabilized a $\beta$ (CSa $\beta$ ) defensin $(95.6 \% \text { confidence score, Phyre })^{23,103,134,216}$. CSa $\beta$ defensins represent a wide-spread and well-characterized group of antimicrobial proteins that are found in many eukaryotes, including mollusks, arachnids, insects, plants and fungi and that exert different antimicrobial activities that almost exclusively rely on their amphipathic protein structure ${ }^{116,217-219}$. Here we study the role of VdAMP3 in the infection biology of $V$. dahliae by assessing the hypothesis that VdAMP3 is used to suppress microbial competitors in host plant tissues.

\section{Results}

As a first step to determine the role of VdAMP3 in $V$. dahliae infection biology, we assessed conditions for expression of the VdAMP3 gene. Transcriptome analysis of diverse $V$. dahliae strains during colonization of a diversity of hosts did not reveal in planta expression of VdAMP3 thus far ${ }^{61,81,87,88}$. In contrast, a literature search revealed that strong induction of this effector gene was recorded during transcriptome analysis of $V$. dahliae strain XS11 grown in vitro during microsclerotia formation ${ }^{93}$. To validate this finding, we analyzed in vitro expression of VdAMP3 in $V$. dahliae strain JR2, which was previously shown to exploit VdAve1 and VdAMP2 for microbiome manipulation ${ }^{134}$. To this end, $V$. dahliae conidiospores were spread on nitrocellulose membranes placed on top of solid minimal medium and fungal material was harvested prior to microsclerotia formation, after 48 hours of incubation, and after the onset of microsclerotia formation, after 96 hours of incubation. Expression of VdAMP3 was determined at both time points with real-time PCR alongside expression of the Chr6g02430 gene that encodes a putative cytochrome P450 enzyme that acts as a marker for microsclerotia formation ${ }^{93,220}$. Consistent with the observations for $V$. dahliae strain XS1193, no VdAMP3 expression was detected at 48 hours when 
also Chr6g02430 was not expressed and no visual microsclerotia formation could be observed on the growth medium (Fig. 1a). However, induction of VdAMP3 as well as Chr6g02430 was observed after 96 hours of incubation, at which time point also the formation of microsclerotia on the growth medium became apparent (Fig. 1a). Collectively these data demonstrate that expression of VdAMP3 coincides with microsclerotia formation in vitro also for $V$. dahliae strain JR2.

a

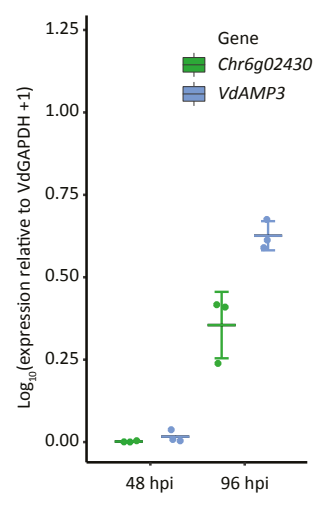

b

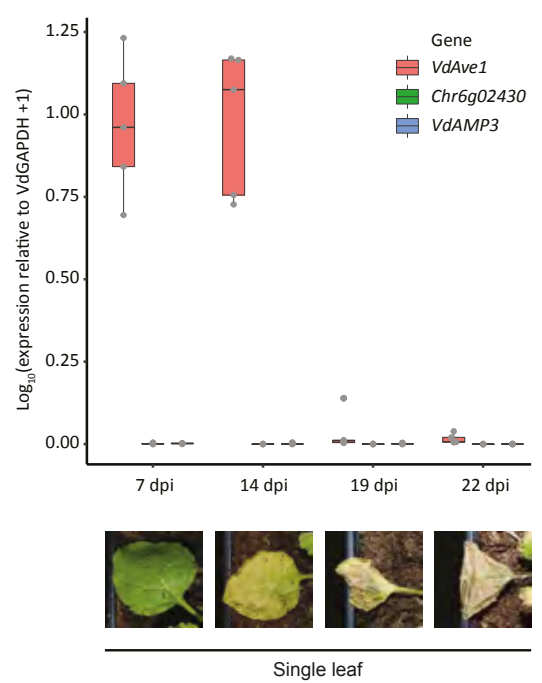

C

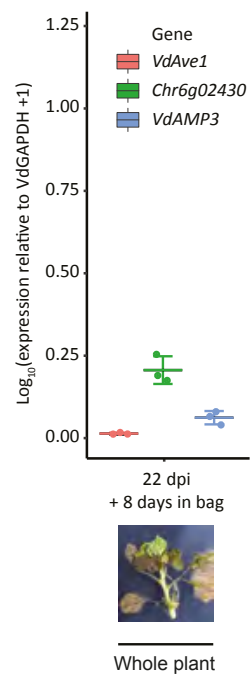

FIGURE 1 | Verticillium dahliae VdAMP3 expression coincides with microsclerotia formation in vitro and in planta. (a) Expression of VdAMP3 and the marker gene for microsclerotia development Chr6g02430, relative to the household gene VdGAPDH at 48 and 96 hours of in vitro cultivation. The plot displays the average expression of three biological replicates \pm SD. (b) Expression of VdAve1, VdAMP3 and Chr6g02430 in N. benthamiana leaves at 7, 14, 19 and 22 days post inoculation (dpi) ( $N=5)$. (c) Expression of VdAve1, VdAMP3 and Chr6g02430 in tissue of N. benthamiana plants harvested at 22 days post inoculation after 8 days of incubation in sealed plastic bags. The plot displays the average expression of three biological replicates \pm SD.

Although previous transcriptome analyses failed to detect in planta expression of VdAMP3, it needs to be realized that these analyses were predominantly performed for relatively early infection stages when the fungus is still confined to the xylem vessels and microsclerotia formation has not been initiated. Consequently, we speculated that in planta expression of VdAMP3 might have been missed. Thus, we inoculated Nicotiana benthamiana with $V$. dahliae and determined expression of VdAMP3 in leaves and petioles sampled at different time points and displaying different disease phenotypes, ranging from asymptomatic at 7 days post inoculation (dpi) to complete necrosis at $22 \mathrm{dpi}$. As expected, a strong induction of $V d A v e 1$ was detected at 7 and

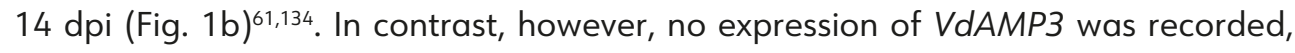


even at the latest time point when the leaf tissue had become completely necrotic (Fig. 1b). Importantly, also no Chr6g02430 expression was detected at any of these time points (Fig. 1b), suggesting that microsclerotia formation had not yet started in these tissues. Indeed, visual inspection of the necrotic plant tissue collected at $22 \mathrm{dpi}$ did not reveal microsclerotia presence. To induce microsclerotia formation, $V$. dahliaeinoculated $N$. benthamiana plants harvested at 22 dpi were incubated in sealed plastic bags and incubated in the dark to increase the relative humidity and mimic conditions that may occur during tissue decomposition in the soil. Interestingly, after 8 days of incubation, when the first microsclerotia could be observed, induction of VdAMP3 as well as Chr6g02430 was detected (Fig. 1c). Collectively, these findings suggest that in planta expression of VdAMP3 coincides with microsclerotia formation, similar to observations previously made in vitro. Moreover, our data suggest that VdAMP3 expression primarily depends on a developmental stage of $V$. dahliae rather than on a host factor such as tissue necrosis.

To determine more precisely where VdAMP3 is expressed, and to improve our understanding of how the effector may contribute to microsclerotia formation, we generated a $V$. dahliae reporter strain expressing eGFP under control of the VdAMP3 promoter. Intriguingly, microscopic analysis of the reporter strain during microsclerotia formation stages in vitro (Fig. 2a), revealed that VdAMP3 is expressed by swollen hyphal cells that act as primordia that subsequently develop into microsclerotia, but not by the adjacent hyphal cells or recently developed microsclerotia (Fig. 2b-d). This highly specific expression of VdAMP3 suggests that the effector protein, through its presumed antimicrobial activity, may facilitate the formation of microsclerotia in the decaying host tissue that is likely a microbially competitive niche due to the presence of decay organisms.

The predicted structural homology of VdAMP3 with CSaß defensins (Phyre², confidence score $95.6 \%)^{103}$ (Fig. 3a) points towards potential antimicrobial activity of the effector protein. To test if VdAMP3 indeed exerts antimicrobial activity, we attempted cloning of the VdAMP3 coding sequence into expression vectors for recombinant protein production using Escherichia coli. However, screening of E. colitransformants repeatedly and exclusively resulted in the identification of mutants carrying a single nucleotide polymorphism responsible for the replacement of one of the cysteine residues. Generally, the stability of CSaß defensins strongly depends on the presence of multiple disulfide bonds which, based on a predicted structure, is likely to be true for VdAMP3 as well (Fig. 3a). We therefore speculated that E. coli is sensitive to the VdAMP3 protein, and that the repeated identification of mutants carrying this nucleotide polymorphism as the only variant that could be cloned is not only indicative for the antibacterial activity of VdAMP3, but also suggests that the mutant encodes an inactivate variant. We subsequently attempted heterologous production using the yeast Pichia pastoris. However, although VdAMP3 could be successfully cloned for recombinant expression, 
yields were insufficient to perform further analyses. As an alternative for recombinant expression, we generated a $V$. dahliae transformant to mediate in vitro expression of VdAMP3 under control of the VdAve1 promoter (Supplementary Fig. 1a). To assess for potential antimicrobial activity, we co-cultivated wild-type V. dahliae and the VdAMP3 expression transformant with two plant endophytes, Bacillus subtilis and Pseudomonas corrugata, of which the former one has previously been characterized as antagonist of $V$. dahliae ${ }^{134}$. A strong reduction in bacterial colony forming units was observed upon 48 hours of co-cultivation with the VdAMP3 expression transformant when compared with co-cultivation with wild-type $V$. dahliae (Fig. 3b,c). Furthermore, filter-sterilized culture filtrates obtained from in vitro grown VdAMP3 expression transformant, but not from wild-type $V$. dahliae, similarly inhibited $P$. corrugata growth. In contrast, neither of the culture filtrates reduced growth of the plant-associated fungus Fusarium oxysporum or the mycoparasite Trichoderma viride. Collectively, these data suggest that VdAMP3 is an antimicrobial protein and that concentrations sufficient to inhibit bacteria do not affect fungal growth.
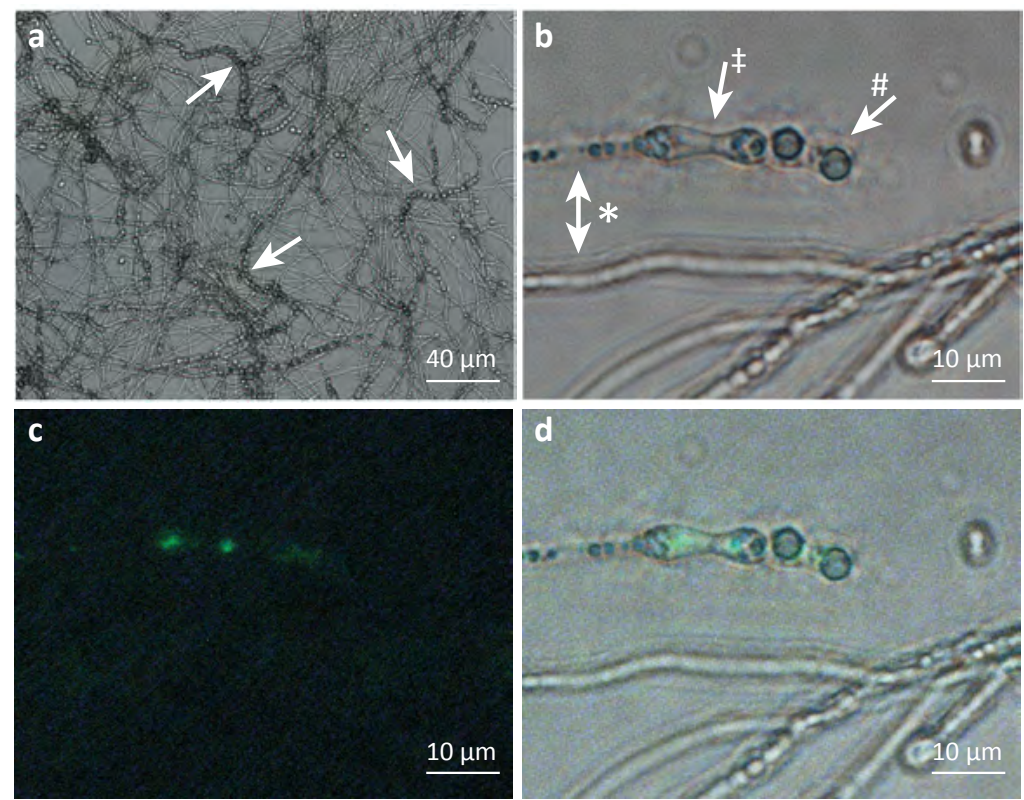

FIGURE 2 | VdAMP3 is specifically expressed in hyphal cells that develop into microsclerotia. (a) Microsclerotia formation of a $p V d A M P 3:: e G F P$ reporter mutant as detected after 7 days of cultivation in Czapek Dox medium. Typical chains of microsclerotia ${ }^{221,222}$ are indicated by arrow heads. (b) Bright field image of various $V$. dahliae cell types after 7 days of cultivation in Czapek Dox, including hyphae ( ${ }^{*}$ ), swollen hyphal cells developing into microsclerotia ( $\ddagger$ ) and mature microsclerotia (\#). (c) GFP signal for the image as shown in (b), indicative for activity of the VdAMP3 promoter, is exclusively detected in the swollen hyphal cells developing into microsclerotia. (d) Overlay of (b) and (c). 
a

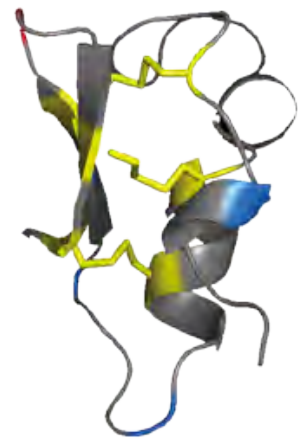

\section{C}

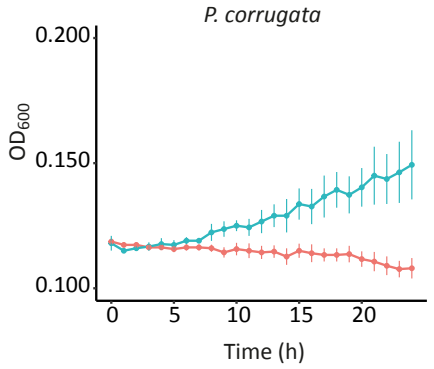

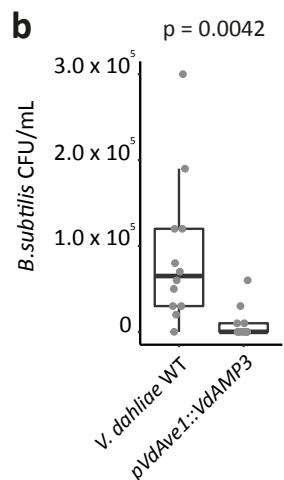

T. viride

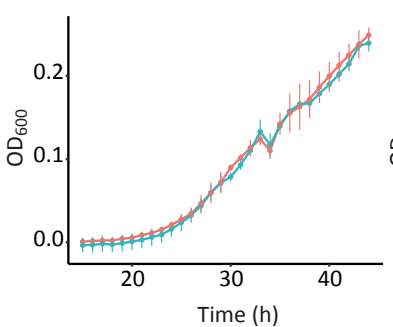

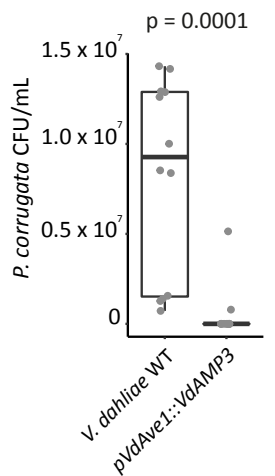

F. oxysporum

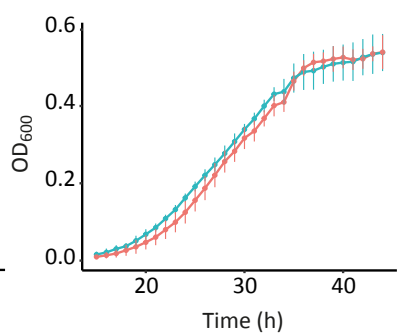

Culture filtrate: $\rightarrow-$ V. dahliae WT $\rightarrow-p$ VdAve1::VdAMP3

FIGURE 3 | VdAMP3 is an antimicrobial protein. (a) The predicted structure of VdAMP3 shares homology with cysteine-stabilized $a \beta(\mathrm{CSa} \beta)$ defensins. The disulfide bonds stabilizing the predicted antiparallel $\beta$-sheets and the a-helix are highlighted in yellow. Positively and negatively charged amino acid residues are highlighted in blue and red, respectively. (b) VdAMP3 exerts antibacterial activity. Growth of $B$. subtilis (left) and $P$. corrugata (right) in the presence of the $p V d A v e 1:: V d A M P 3$ transformant strongly reduces the number of bacterial colony forming units when compared with growth in the presence of wild type $V$. dahliae as determined by plating after 48 hours of co-cultivation (unpaired two-sided student's t-test; $N=12$ ). (c) VdAMP3 affects bacterial, but not fungal, growth. Growth of $P$. corrugata, $T$. viride and $F$. oxysporum in filter-sterilized culture filtrates from wild-type $V$. dahliae and the VdAMP3 expression transformant grown in liquid $0.2 \times \mathrm{PDB}+0.5 \times \mathrm{MS}$ medium. Graphs display the average $\mathrm{OD}_{600}$ of three biological replicates $\pm \mathrm{SD}$.

The antibacterial activity of $\mathrm{VdAMP3}$, combined with its expression during microsclerotia formation, points towards a role in microbial competition during advanced $V$. dahliae infection stages when microsclerotia formation takes place. To study the importance of VdAMP3 during and after host colonization, VdAMP3 deletion mutants were generated as well as complementation strains (Supplementary Fig. 1b). Importantly, targeted deletion of VdAMP3 did not affect in vitro growth nor microsclerotia formation (Fig. $4 a, b)$.

To determine if VdAMP3 contributes to Verticillium wilt disease development, $N$. benthamiana plants were inoculated with wild-type $V$. dahliae and the VdAMP3 deletion mutant. In line with our inability to detect expression during early infection 
stages, disease phenotypes and $V$. dahliae biomass quantification using real-time PCR did not reveal a contribution of VdAMP3 to host colonization up to two weeks after inoculation (Fig. 5a,b). To test if VdAMP3 contributes to V. dahliae niche establishment following systemic host colonization, we harvested the N. benthamiana plants and sealed them in plastic bags to induce microsclerotia formation. Interestingly, following four weeks of incubation, $V$. dahliae biomass quantification in N. benthamiana plants inoculated with the various genotypes using real-time PCR revealed a significant reduction in biomass of the VIAMP3 deletion mutant when compared with wild-type $V$. dahliae and complementation mutants (Fig $5 c, d$ ).

To investigate if the effects of VdAMP3 are limited to N. benthamiana, or whether those also extend to other hosts, we inoculated Arabidopsis thaliana plants with wildtype $V$. dahliae and the VdAMP3 deletion mutant. Consistent with our observations for $N$. benthamiana, deletion of VdAMP3 did not affect establishment of Verticillium wilt in A. thaliana (Supplementary Fig. 2a,b). However, V. dahliae biomass quantification in above-ground $A$. thaliana tissues at three weeks post inoculation revealed reduced accumulation of $V$. dahliae in the absence of VdAMP3 (Supplementary Fig. 2c). Thus, VdAMP3 contributes to $V$. dahliae niche establishment in multiple hosts.

a

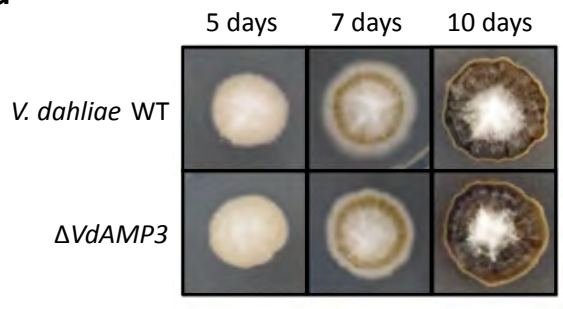

b

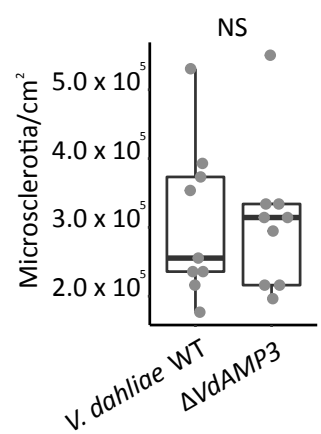

FIGURE 4 | Deletion of VdAMP3 does not affect V. dahliae microsclerotia formation in vitro. (a) Morphology of wild-type $V$. dahliae and the VdAMP3 deletion mutant following five, seven and ten days of in vitro growth on PDA. (b) Deletion of VdAMP3 does not impair microsclerotia formation. After ten days, the colonies as shown in (a) were processed and the number of microsclerotia per $\mathrm{cm}^{2}$ was determined using a haemocytometer. No significant difference in microsclerotia formation was observed (unpaired two-sided student $\mathrm{t}$-test $\mathrm{N}=9$ ). 
a

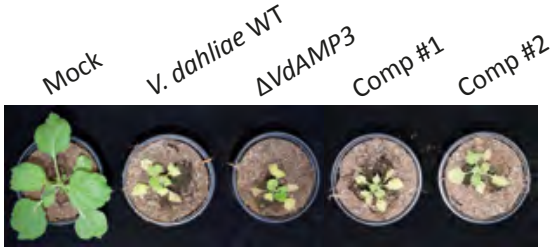

b

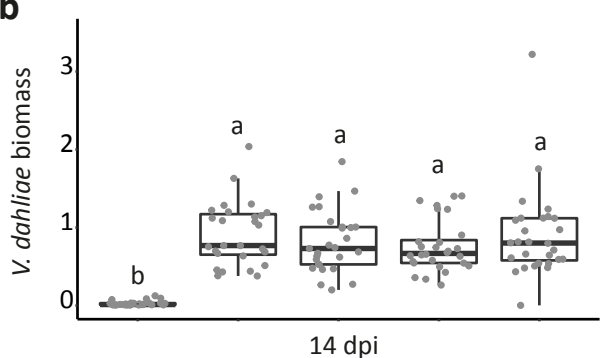

C

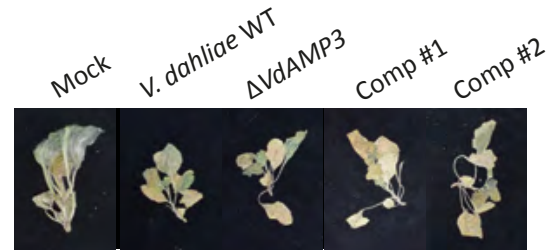

d

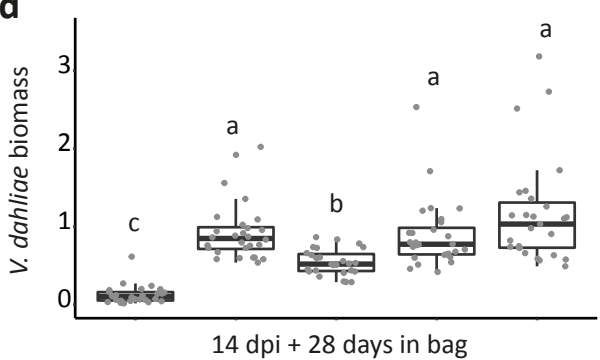

FIGURE 5 | VdAMP3 facilitates V. dahliae microsclerotia formation following systemic host colonization. (a) VdAMP3 does not contribute to establishment of Verticillium wilt disease in $N$. benthamiana. Photos display representative phenotypes of $N$. benthamiana plants infected by wild-type $V$. dahliae (WT), the VdAMP3 deletion ( $\triangle V$ dAMP3) and two complementation (Comp) mutants 14 days post inoculation. (b) Relative $V$. dahliae biomass in above-ground $N$. benthamiana tissues determined with real-time PCR. Different letter labels represent significant differences (one-way ANOVA and Tukey's posthoc test; $p<0.05 ; N \geq 27$ ) (c) Representative phenotypes of $N$. benthamiana plants as shown in (a) after 28 days of incubation in plastic bags. (d) Relative $V$. dahliae biomass in $N$. benthamiana tissues as displayed in (e). Letters represent significant differences (one-way ANOVA and Tukey's post-hoc test; $p<0.05 ; N \geq 27$ ).

\section{Discussion}

Like all plant associated microbes, $V$. dahliae secretes a multitude of effector molecules to support host colonization ${ }^{5,25,214,215}$. We recently showed that $V$. dahliae not only exploits effector proteins to promote colonization through manipulation of host immune responses or other elements of host physiology, but also to target microbial competitors in- and outside the host in a life-stage dependent manner ${ }^{134}$. More specifically, we revealed that the antibacterial effector VdAMP2 exclusively facilitates niche colonization in the soil prior to host ingress and that another antibacterial effector, $\mathrm{VdAve1}$, besides soil colonization, also promotes host colonization through the active suppression of microbial competitors encountered in the plant microbiome ${ }^{134}$. Arguably, during its infection process, V. dahliae successively colonizes distinct niches where it is likely to encounter different microbial communities. In this study, we characterized a novel effector of $V$. dahliae with antimicrobial activity, the CSaß defensin-like protein VdAMP3.

Transcriptional analyses of $V$. dahliae growing in vitro and in planta revealed that expression of $V d A M P 3$ coincides with microsclerotia formation, which pointed towards 
a role of VdAMP3 during advanced infection stages. Antimicrobial effector molecules can be anticipated to fulfill various roles during advanced infection stages. For instance, effectors might be essential to withstand the increased exposure to microbes and to facilitate niche transition from the vasculature to the host phyllosphere, where the fungus is likely to encounter opportunistic microbes that flourish on the decaying tissue as host immune responses dissipate. Consequently, V. dahliae can be anticipated to actively suppress such opportunists through antimicrobials to establish niche colonization. The successful establishment and protection of a niche during advanced infection stages is crucial to safeguard the formation of viable microsclerotia in turn, which are the propagules to establish the next generation in future. To this end, $V$. dahliae might boost the secretion of antimicrobials during or prior to microsclerotia formation. Indeed, the cell type-specific expression of VdAMP3 during microsclerotia development, combined with the identified contribution of the protein to $V$. dahliae biomass accumulation in decaying host tissue, strongly suggest that $V$. dahliae exploits VdAMP3 to safeguard the formation of microsclerotia.

Interestingly, our in planta expression analyses did not reveal simultaneous expression of VdAve 1 and VdAMP3 (Fig 1b,c). Previous V. dahliae transcriptome analyses repeatedly identified VdAve1 as one of the most highly expressed effector genes in planta and under in vitro growth conditions $\mathrm{s}^{61,81,87,88}$. Consistent with these findings, we detected high expression of VdAve 1 in asymptomatic and chlorotic $N$. benthamiana leaves at 7 and 14 days post inoculation, respectively. However, we detected an unanticipated repression of VdAve1 in necrotic N. benthamiana tissues from 19 days post inoculation onwards (Fig $1 \mathrm{~b}, \mathrm{c}$ ). During these advanced infection stages, $V$. dahliae exits the host vasculature and colonizes the decaying leaf mesophyll. This niche transition is likely accompanied by an increased exposure to microbes, as the necrotic leaf tissue has less active defense, is more nutrient-rich and more accessible to opportunistic microbes than the relatively nutrient-poor xylem vessels ${ }^{223,224}$. Possibly, the different microbial communities and abiotic conditions require different antimicrobial effector molecules from $V$. dahliae to establish itself in these divergent niches. Although the activity spectrum of VdAMP3 requires further characterization, ideally using purified effector protein, antimicrobial activity assays using the VdAMP3 expression transformant, or the culture filtrate obtained through in vitro cultivation of this transformant, revealed a strong activity of VdAMP3 on P. corrugata. In contrast, antimicrobial activity assays using purified $V \mathrm{~V} A \mathrm{ve} 1$ revealed no clear inhibition of $P$. corrugata growth ${ }^{134}$, suggesting that VdAve1 and VdAMP3 have divergent activity spectra.

Although the thick melanized cell walls of microsclerotia may provide sufficient protection against the majority of fungi and bacteria encountered in the soil225,226, antimicrobial effector molecules might also be required to fend off soil-dwelling parasites such as fungivorous nematodes or protists. Possibly, the focused expression 
of $V d A M P 3$ allows $V$. dahliae to coat or load its microsclerotia with the effector protein in order to promote their survival. Along these lines, and given the longevity of microsclerotia, it is interesting that according to its predicted structure, VdAMP3 is likely to be homologous to CSa $\beta$ defensins. Importantly, CSa $\beta$ defensins are highly stable and tightly folded proteins that can withstand adverse conditions such as high temperatures without losing their functionality or structural properties ${ }^{114,227}$. Some CSaß defensins are known to be deposited in plant seeds where they remain stable until their release upon rupture of the seed coat due to seed germination to protect the emerging germling against surrounding microbes ${ }^{115}$. Intriguingly, a 10 minute heat treatment at $100^{\circ} \mathrm{C}$ of the CSa $\beta$ defensins Rs-AFP1 and Rs-AFP2 isolated from radish seeds was previously shown to not affect the antimicrobial activity of the proteins ${ }^{114}$, which exemplifies the potential stability of proteins belonging to this family. Consequently, deposition of VdAMP3 in or on microsclerotia may enhance the survivability of $V$. dahliae during long periods of dormancy in the soil.

In conclusion, our findings demonstrate that $V$. dahliae employs VdAMP3 to protect the formation of microsclerotia, and possibly also to promote their long-term survival in the soil upon tissue decomposition. Moreover, our findings support the hypothesis that $V$. dahliae exploits different antimicrobial effector proteins at different stages of its life cycle.

\section{Materials and methods}

Gene expression analyses. In vitro cultivation of $V$. dahliae strain JR2 for analysis of VdAMP3 and Chr6g02430 expression was performed as described previously ${ }^{93}$. Additionally, for in planta expression analyses, total RNA was isolated from single leaves or complete $N$. benthamiana plants harvested at different time points after $V$. dahliae root dip inoculation. To induce microsclerotia formation, $N$. benthamiana plants were harvested at $22 \mathrm{dpi}$ and incubated in sealed plastic bags (volume $=500 \mathrm{~mL}$ ) for 8 days, prior to RNA isolation. RNA isolations were performed using the Maxwell ${ }^{\odot}$ 16 LEV Plant RNA Kit (Promega, Madison, USA). Real-time PCR was performed as described previously using the primers listed in supplementary Table ${ }^{161}$.

Generation of $\boldsymbol{V}$. dahliae mutants. The VdAMP3 deletion and expression mutants, as well as the eGFP expression mutant, were generated as described previously using the primers listed in supplementary Table $1^{134}$. To generate the VdAMP3 complementation construct, the VdAMP3 coding sequence was amplified with flanking sequences ( 0.9 $\mathrm{kb}$ upstream and $\sim 0.8 \mathrm{~kb}$ downstream) and cloned into $\mathrm{pCG}^{228}$. Finally, the construct was used for Agrobacterium tumefaciens-mediated transformation of $V$. dahliae as described previously ${ }^{96}$. In vitro growth and microsclerotia production of the various $V$. dahliae mutants was tested and quantified as described previously ${ }^{134}$. 
In vitro microbial growth assays and co-cultivation assays. In vitro microbial growth assays were performed using culture filtrates obtained from $V$. dahliae strain JR2 and the VdAMP3 expression mutant as described previously ${ }^{134}$. For the microbial cocultivation assays, conidiospores of $V$. dahliae strain JR2 and the VdAMP3 expression mutant were harvested from PDA plate and diluted to a final concentration of $10^{4}$ conidiospores $/ \mathrm{mL}$ in $0.2 \times$ PDB $+0.5 \times$ Murashige \& Skoog medium (Duchefa, Haarlem, The Netherlands). Subsequently, $2 \mathrm{~mL}$ of the conidiospore suspensions was aliquoted in clear 12 well flat bottom polystyrene tissue culture plates and incubated at $22^{\circ} \mathrm{C}$ and $120 \mathrm{rpm}$ for 64 hours. Next, overnight cultures of the bacterial strains $B$. subtilis AC95 and $P$. corrugata $C 26^{134}$ were added to wells to a final $\mathrm{OD}_{600}=0.05$. After 48 hours of incubation at $22^{\circ} \mathrm{C}$ and $120 \mathrm{rpm}$, the microbial suspensions were diluted and plated on LB to quantify the bacterial colony forming units per $\mathrm{mL}$.

Disease assays. Three-week-old N. benthamiana seedlings grown in the greenhouse at $21^{\circ} \mathrm{C} / 19^{\circ} \mathrm{C}$ during $16 \mathrm{~h} / 8 \mathrm{~h}$ day/night periods, respectively, with $70 \%$ relative humidity, were inoculated with $V$. dahliae through root-dip inoculation as described previously ${ }^{59}$. After 14 days, above-ground parts of the $N$. benthamiana plants were harvested and stored at $-20^{\circ} \mathrm{C}$. Alternatively, above-ground parts were collected and transferred to plastic bags (volume $=500 \mathrm{~mL}$ ) and incubated for four weeks at room temperature. Next, all N. benthamiana samples were ground using mortar and pestle. Subsequent genomic DNA isolation and $V$. dahliae biomass quantification was performed as previously described using the primers listed in Supplementary Table $1^{229}$.

Fluorescence microscopy. Spores of the pVdAMP3::eGFP reporter strain were harvested from a PDA plate and diluted to a final concentration of $10^{5}$ conidiospores/ $\mathrm{mL}$ in $0.1 x$ Czapek Dox medium. The suspension was incubated for one week at room temperature to allow hyphae to grow and microsclerotia to form. Finally, eGFP accumulating in the fungal cells was detected using a Nikon ECLIPSE 90i microscope.

\section{Acknowledgements}

B.P.H.J.T. acknowledges support from the Research Council Earth and Life Sciences (ALW) of the Netherlands Organization of Scientific Research (NWO) and from the Deutsche Forschungsgemeinschaft (DFG, German Research Foundation) under Germany's Excellence Strategy - EXC 2048/1 - Project ID: 390686111. 


\section{Supplementary data}

a

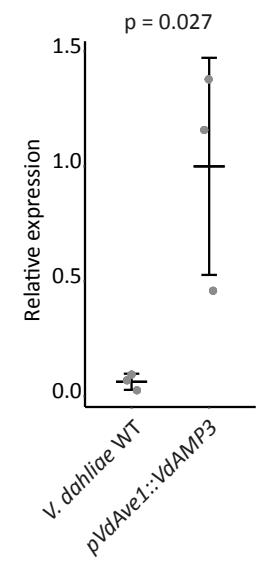

b

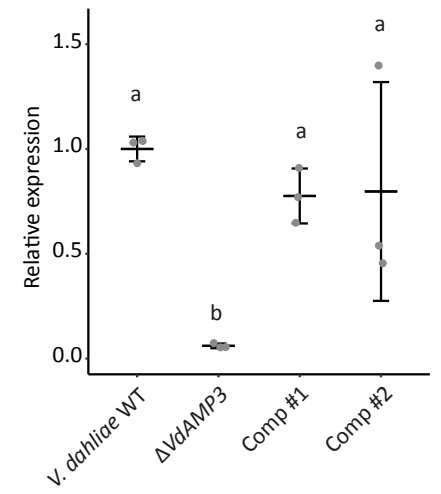

SUPPLEMENTARY FIGURE 1 | Expression of VdAMP3 in V. dahliae mutants. (a) Expression of VdAMP3 relative to VdGAPDH in wild-type $V$. dahliae and the $p V d A v e 1:: V d A M P 3$ transformant after five days of cultivation in $0.2 \times$ PDB $+0.5 \times$ Murashige \& Skoog (MS) medium (unpaired two-sided student's t-test; $N=3$ ). The plot displays the average expression \pm SD. (b) Expression of VdAMP3 relative to VdGAPDH during microsclerotia formation by $V$. dahliae WT and the VdAMP3 deletion and complementation mutants after 7 days of cultivation in Czapek Dox medium. Letters represent statistically significant differences (one-way ANOVA and Tukey's post-hoc test; $p<0.05 ; \mathrm{N}=3$ ). The plot displays the average expression \pm SD. 
a

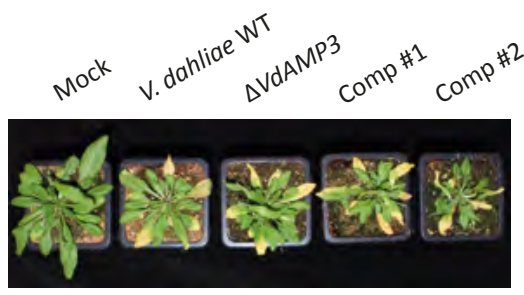

b

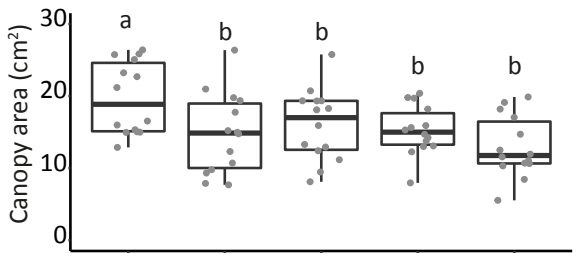

C

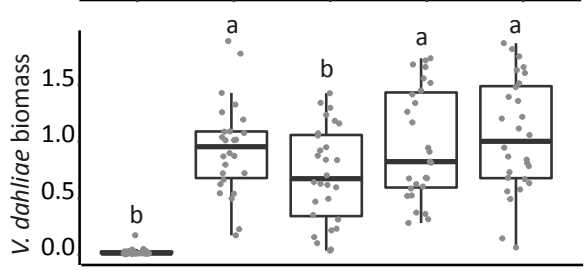

SUPPLEMENTARY FIGURE 2 | VdAMP3 contributes to V. dahliae biomass accumulation in Arabidopsis thaliana but does not influence development of disease symptoms. (a) Deletion of VdAMP3 does not affect establishment of Verticillium wilt disease in A. thaliana. Photos display representative phenotypes of A. thaliana plants 21 days post inoculation with $V$. dahliae WT and VdAMP3 deletion and complementation mutants. (b) Canopy area of $A$. thaliana plants inoculated by the different $V$. dahliae genotypes. Letter labels represent statistically significant differences when compared with $V$. dahliae WT (unpaired two-sided student's t-test; $N=14$ ). (c) Relative $V$. dahliae biomass in above-ground $A$. thaliana tissues determined with real-time PCR. Letter labels represent statistically significant differences when compared with $V$. dahliae WT (unpaired two-sided student's t-test; $\mathrm{p}<0.05 ; \mathrm{N} \geq 26$ ). 
SUPPLEMENTARY TABLE 1 | Primers used in this study.

\begin{tabular}{|c|c|c|}
\hline Name & Sequence $\left(5^{\prime}-->3^{\prime}\right)$ & Application \\
\hline VdAve1_qPCR_Fw & TGTTACCAAAGCAGCACACAAGG & Real-time PCR \\
\hline VdAve1_qPCR_Rv & CCTTATGCCTCGTTCCCTTCCAC & Real-time PCR \\
\hline VdGAPDH_Fw & CGAGTCCACTGGTGTCTTCA & Real-time PCR \\
\hline VdGAPDH_Rv & CCCTCAACGATGGTGAACTT & Real-time PCR \\
\hline VdAMP3_qPCR_Fw & ATGAAGCTCATTTCTGC & Real-time PCR \\
\hline VdAMP3_qPCR_Rv & CTAGTTGCAAATGCACAC & Real-time PCR \\
\hline Chr6g02430_qPCR_Fw & CAGAGCACCACTCACCACAT & Real-time PCR \\
\hline Chr6g02430_qPCR_Rv & ATCAGGAGTGGCGTGAAGTC & Real-time PCR \\
\hline ITS1-Fw & AAAGTTTTAATGGTTCGCTAAGA & Real-time PCR \\
\hline St-Ve1-Rv & CTTGGTCATTTAGAGGAAGTAA & Real-time PCR \\
\hline NbRUB_Fw & TCCGGGTATTAGCAAAAGCGT & Real-time PCR \\
\hline NbRUB_Rv & CCCAAGATCTCGGTCAGAGC & Real-time PCR \\
\hline AtRUB_Fw & GCAAGTGTTGGGTTCAAAGCTGGTG & Real-time PCR \\
\hline AtRUB_Rv & $\begin{array}{l}\text { CCAGGTTGAGGAGTTACTCGGAAT- } \\
\text { GCTG }\end{array}$ & Real-time PCR \\
\hline Pacl_VdAMP3_FT_Fw & $\begin{array}{l}\text { CGGTATTTAATTAAATGAAGCTCATTT } \\
\text { CTGCAACTAGC }\end{array}$ & $\begin{array}{l}\text { To generate pVdAve1::VdAMP3 } \\
\text { transformant }\end{array}$ \\
\hline VdAMP3_Notl_RV & $\begin{array}{l}\text { CGTCTAGCGGCCGCCTAGTTGCAA } \\
\text { ATGCACACAG }\end{array}$ & $\begin{array}{l}\text { To generate pVdAve } 1:: \text { VdAMP3 } \\
\text { transformant }\end{array}$ \\
\hline JR2_VdAMP3_LB_Fw & $\begin{array}{l}\text { GGTCTTAAUTTTGAGGGGTTCAGC } \\
\text { CGATG }\end{array}$ & To generate VdAMP3 deletion mutant \\
\hline JR2_VdAMP3_LB_Rv & $\begin{array}{l}\text { GGCATTAAUGACGATATGAGTGCTT } \\
\text { GCGG }\end{array}$ & To generate VdAMP3 deletion mutant \\
\hline JR2_VdAMP3_RB_Fw & $\begin{array}{l}\text { GGACTTAAUAATGCTTGAGATGAC } \\
\text { GACGC }\end{array}$ & To generate VdAMP3 deletion mutant \\
\hline JR2_VdAMP3_RB_Rv & $\begin{array}{l}\text { GGGTTTAAUCTGCTCACCAAGCCT } \\
\text { CCTTC }\end{array}$ & To generate VdAMP3 deletion mutant \\
\hline VdAMP3_Comp_Fw & $\begin{array}{l}\text { GGGGACAGCTTTCTTGTACAAAGTG } \\
\text { GTTTGAGGGGTTCAGCCGATG }\end{array}$ & $\begin{array}{l}\text { To generate VdAMP3 complementation } \\
\text { mutant }\end{array}$ \\
\hline VdAMP3_Comp_Rv & $\begin{array}{l}\text { GGGGACAACTTTGTATAATAAAGTT } \\
\text { GCTGCTCACCAAGCCTCCTTC }\end{array}$ & $\begin{array}{l}\text { To generate VdAMP3 complementation } \\
\text { mutant }\end{array}$ \\
\hline Promoter_VdAMP3_Fw & $\begin{array}{l}\text { CTCGGAATTAACCCTCACTAAAGGGA } \\
\text { ACAAAAGCTGGAGCTCACACAACAT } \\
\text { CTATGCTTCAGAAGGTGGCAAAAG } \\
\text { TG }\end{array}$ & $\begin{array}{l}\text { To generate pVdAMP3::eGFP } \\
\text { transformant }\end{array}$ \\
\hline Promoter_VdAMP3_Rv & $\begin{array}{l}\text { ATGATGGCCATGTTATCCTCCTCGCC } \\
\text { CTTGCTCACCATATTAATTAAGATTGAT } \\
\text { GGTGTCAAGAGGGTCTGGGATATG } \\
\text { ATTG }\end{array}$ & $\begin{array}{l}\text { To generate pVdAMP3::eGFP } \\
\text { transformant }\end{array}$ \\
\hline
\end{tabular}




\section{Chapter 7}

General discussion

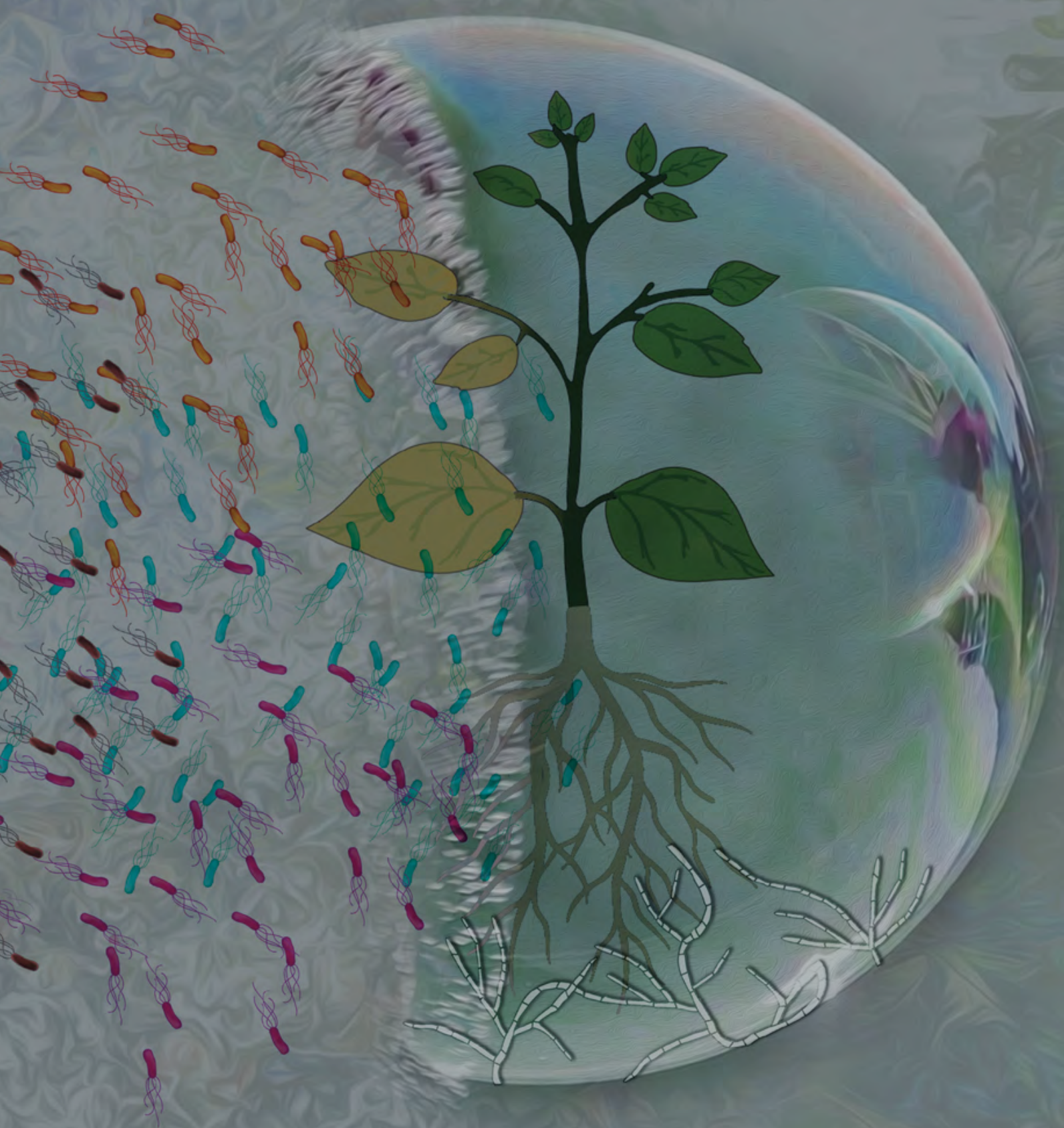




\section{Introduction}

Since the beginning of this century, the general picture has emerged that plant pathogenic microbes secrete a plethora of effector molecules to promote colonization of their hosts ${ }^{2}$. Depending on the scientist whom one asks and the definition (s)he maintains, plant pathogen effector catalogs comprise molecules of various kinds that are involved in a broad-range of processes. However, everyone will acknowledge that effectors at least comprise small cysteine-rich in planta-secreted proteins involved in the subversion of host immune systems $s^{5,25,214,215}$. So far, the study of effector proteins has largely been confined to the binary interactions between pathogens and their hosts. However, plants associate with countless microbes, collectively termed their microbiota, which represents an important determinant for their well-being. Aboveground as well as below-ground plant organs are colonized by beneficial microbes that aid in the alleviation of various stresses ${ }^{11,21,85,230,231}$. Importantly, plants are capable to define their own microbiota compositions and recruit beneficial microbes that suppress pathogenic invaders ${ }^{15,20}$. Hence, the plant microbiome, i.e. the microbes, their genomes and their environment ${ }^{6}$, can be considered an inherent, exogenous, layer that extends the endogenous innate immune system. Considering the protective role of beneficial microbiomes, we speculated at the onset of this $\mathrm{PhD}$ research project that disease establishment by a plant pathogenic microbe can be anticipated to be the result of a successful interaction with its host as well as with the host-associated microbiota (Chapters $1 \& 2)^{78}$, collectively termed the plant holobiont. To this end, we further hypothesized that plant pathogens exploit effector molecules also for the manipulation of host microbiome compositions in order to facilitate disease establishment (Chapter 2 ) $^{78}$. In this chapter, I discuss the results obtained in this thesis, and the presently described and the anticipated roles of fungal plant pathogen effector proteins in microbiome manipulation in a broader context.

\section{Effector-mediated microbiome manipulation by soil-borne pathogens}

The soils in which plants grow represent some of the most biologically diverse and microbe-rich environments on our planet. Soil-dwelling microbes are continuously engaged in fierce competition with each other for limited nutrients and space ${ }^{12}$. Consequently, the ability to establish a niche in the microbially competitive soil can be anticipated to largely depend on molecular mechanisms involved in the suppression of microbial co-inhabitants on the one hand, and the protection from antimicrobials secreted by these microbes on the other hand. Recent metagenome analyses of socalled "topsoils" collected across the globe indeed provided evidence that microbial antagonism is ubiquitous in soil ${ }^{213}$. 
While many soil-inhabiting microbes display no host-associated lifestyles, and therefore predominantly encounter other free-living microbes, soil-borne plant pathogens are additionally exposed to a wealth of plant-associated microbes. Especially the rhizosphere, the narrow zone of soil in close proximity to the roots where plants deposit their carbon- and nitrogen-rich exudates, represents a hostile environment with high microbial densities ${ }^{10,13}$. Importantly, plants define their root microbiome compositions using these exudates, and recruit beneficial microbial communities to suppress pathogen invasion $15,20,21,230$. Accordingly, we hypothesized that soil-borne plant pathogens dedicate part of their effector repertoires to the manipulation of such beneficial microbial communities in order to facilitate disease establishment (Chapter 2$)^{78}$. In this thesis research we provided the first proof-of-concept evidence for this hypothesis, as we showed that the previously identified virulence effector VdAve1 that is secreted by the soil-borne xylem invading plant pathogenic fungus $V$. dahliae is an antibacterial protein that impacts host microbiome compositions to promote host colonization ${ }^{61,134}$ (Fig. 1a,b) (Chapter 3). More specifically, we demonstrated that secretion of VdAve1 by $V$. dahliae suppresses the proliferation of antagonistic bacteria in planta, including Sphingomonads, and hence promotes Verticillium wilt disease development (Chapter $3)^{134}$. Moreover, we showed that $\mathrm{VdAve} 1$ is also highly expressed outside the host and contributes to $V$. dahliae soil colonization (Chapter 3$)^{134}$. Furthermore, when we probed the predicted $V$. dahliae secretome for proteins with putative structural homology to known antimicrobial proteins (AMPs), we identified ten additional effector candidates that are potentially involved in microbiome manipulation (Chapter 3$)^{134}$. Thus far, two of these candidates, named VdAMP2 and VdAMP3, have been characterized as AMPs aiding in the microbial competition of $V$. dahliae with the various microbial communities that are encountered during its life cycle (Chapters $3 \& 6$ ). VdAMP2 shares structural homology with the amphipathic $\beta$-hairpins of aerolysin-type $\beta$-pore forming toxins ( $\beta$-PFTs) and is exclusively expressed in the soil where it facilitates niche colonization together with VdAve1 (Chapter 3) ${ }^{134}$ (Fig. 1c). VdAMP2 and VdAve1 display divergent activity spectra, suggesting that their activities complement each other for optimal $V$. dahliae soil colonization (Chapters 3 ). While the mode of action of VdAMP2 remains presently unknown, in-depth characterization uncovered VdAve1 as a novel lysozyme (Chapter 4 \& 5). Finally, VdAMP3 is a defensin-like antimicrobial effector protein that was shown to safeguard the formation of $V$. dahliae resting structures, so-called microsclerotia, in the decaying host phyllosphere following systemic colonization and was speculated to promote the long-term survival of the resting structures in the soil (Chapter 6) (Fig. 1d). Importantly, thus far, the role of these microbiome-manipulating $V$. dahliae effectors has been exclusively studied under controlled greenhouse conditions in plants grown on potting soil, and consequently the relevance of these effectors in ecologically more relevant contexts remain to be demonstrated. Nevertheless, the proof-of-concept findings presented in this thesis strongly suggest that $V$. dahliae exploits these effector proteins to target microbiomes that it encounters in nature. 
Most likely, the three effector proteins characterized in this thesis research only represent a small proportion of all $V$. dahliae effectors intended for microbiome manipulation. Eight of the effector proteins that are predicted to share structural homology with known AMPs have remained uncharacterized till date, but can be anticipated to comprise additional effectors involved in microbial interactions. Additionally, the secretome of $V$. dahliae is likely to comprise proteins with novel antimicrobial folds or domains, are thus far likely disqualified as candidates based on their structural models. It is important to note that also VdAve1 shares no sequence or structural homology with previously described AMPs, and thus the effector protein was initially not anticipated to exert antimicrobial activity. However, its ubiquitous expression combined with our inability to purify the protein following expression in E. coli prompted us to address its potential antimicrobial activity, which eventually resulted in its characterization as a novel type of lysozyme (Chapter 5).

Given the microbially competitive nature of soils and root microbiota, it can be stated beyond doubt that $V$. dahliae will not be the only soil-borne pathogen that exploits its effector protein catalog for microbiome manipulation. Several metagenome analyses have revealed an impact on root microbiome compositions upon colonization by soil-borne pathogens ${ }^{21,232,233}$. However, such community structure alterations have predominantly been assessed in the light of enrichment for beneficial microbes that may be involved in disease suppression. Presently, evidence for the involvement of effector proteins in shaping these microbial interactions, other than the ones characterized for $V$. dahliae, is exclusively based on transcriptional analysis of genes that are hypothesized to play roles in intermicrobial interactions. For instance, transcriptome analyses performed upon confrontation of the cereal pathogen Bipolaris sorokiniana with the beneficial root endophyte Serendipita vermifera in soil and in planta revealed that both fungi express multiple genes encoding potential microbe-targeting effectors, including putative chitinases ${ }^{234}$. Future research is needed to provide insight in the conservation of effector-mediated host microbiome manipulation as a strategy of soil-borne plant pathogens to promote host colonization. 


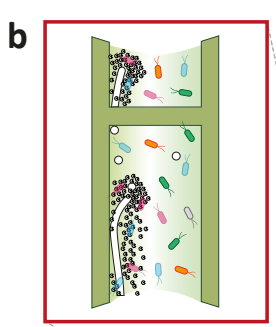

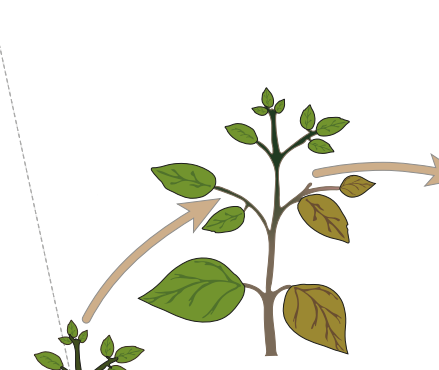

III a

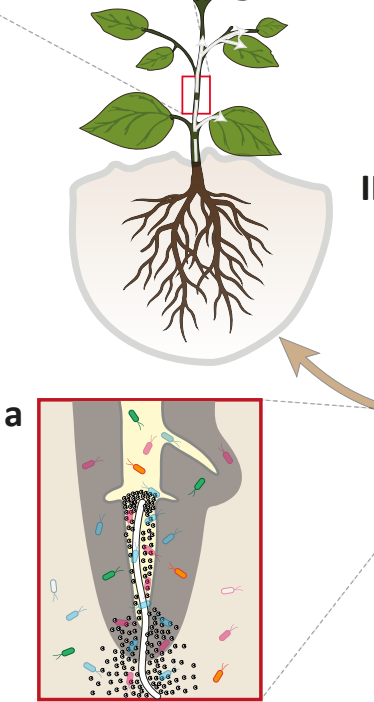

d

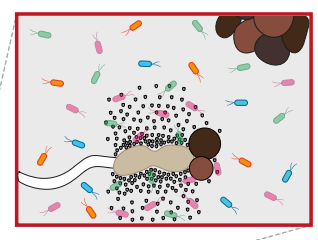

II

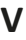

IV
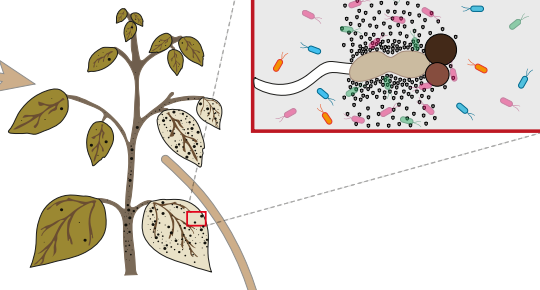

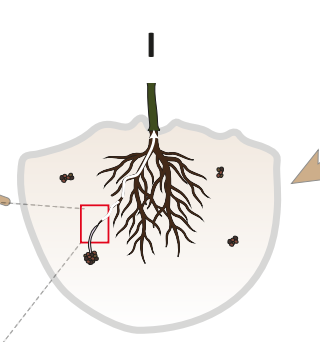

C

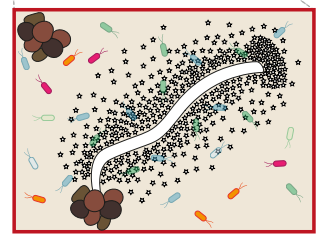

Figure 1. Life stage-specific effector-mediated microbiome manipulation by the soil-borne broad host-range fungal plant pathogen Verticillium dahliae. The fungus survives in the soil through multicellular melanized resting structures named microsclerotia. Germination of these microsclerotia is stimulated by plant root exudates, after which the emerging hyphae grow saprophytically through the soil and rhizosphere to penetrate plant roots (I). Next, the fungus crosses the root cortex and enters the xylem vessels where sporulation occurs (II). The conidiospores are transported with the sap stream in the vasculature to distal plant tissues. Once conidiospores get trapped germination occurs, after which the fungus penetrates into new xylem vessels where sporulation re-occurs. This systemic colonization is accompanied by typical Verticillium wilt symptoms, including chlorosis, necrosis, and wilting (III). Once the tissue starts to senesce, $V$. dahliae emerges from the vasculature to colonize decaying host tissues where new microsclerotia are produced (IV). These microsclerotia are released into the soil upon tissue decomposition (V). V. dahliae secretes a plethora of effectors to promote host colonization, several of which target the various microbiota that it encounters during its life cycle. VdAve1 is a ubiquitously expressed lysozyme that promotes colonization of the soil (a) as well as the roots (a) and xylem vessels (b) of its hosts through selective suppression of antagonistic bacteria (see Chapter 3-5 for details). (c) VdAMP2 is an antibacterial effector that is exclusively expressed in the soil where it complements the activity spectrum of $\mathrm{VdAve} 1$ to mediate niche colonization (see Chapter 3 for details). (d) Finally, VdAMP3 is a defensin-like antibacterial effector protein that is specifically expressed in the hyphal sections that develop into microsclerotia to mediate the formation of these resting structures (see Chapter 6 for details). 


\section{Effector-mediated microbiome manipulation in the host phyllosphere}

The phyllosphere comprises all aerial parts of a plant that, similar to the roots, are colonized by a diversity of microbes ${ }^{7,85}$. Although the influence of plants on the community structure of phyllosphere microbiota is presently less clear, it has become evident that phyllosphere inhabitants positively impact plant health and, for instance, contribute to disease suppression ${ }^{7,8,231,235-238}$. Irrespective of their lifestyles, disease establishment by fungal pathogens in the phyllosphere of compatible host plants is typically initiated by individual spores that land on these host tissues following their dispersal. Next, these spores germinate and the emerging hyphae start to colonize the exterior of their hosts, the so-called episphere. Arguably, antimicrobial effectors might a play a crucial role both during, and right after, spore germination as they could boost initial niche colonization in the established microbial communities of the phyllosphere through the suppression of epiphytes in the immediate environment. Accordingly, the fungal wheat pathogen Zymoseptoria tritici was previously shown to induce the expression of the effector gene $Z t 6$, encoding a phytotoxic and antimicrobial secreted ribonuclease, during spore germination on the surface of wheat leaves ${ }^{42}$. Hence, $\mathrm{Zt} 6$ was speculated to clear the immediate surroundings of the germinating spore from microbial competitors to safeguard initial leaf colonization. However, targeted deletion of $Z t 6$ did not affect $Z$. tritici disease establishment, and the role of $Z \mathrm{t} 6$ in microbial inhibition in planta has remained unclear. Nevertheless, is seems likely that pathogens exploit microbe-targeting effectors to establish colonization of the episphere.

Following epiphytic colonization of host tissues in the phyllosphere, plant pathogen infection typically continues in the apoplast where, depending on the lifestyle, plant pathogens adopt different strategies to acquire nutrients from their hosts. While biotrophs only obtain nutrients from living plant tissue, necrotrophs actively kill host cells for nutrient acquisition. However, most pathogens can be placed somewhere in the continuum in between these lifestyles and are classified as hemibiotrophs ${ }^{239}$ that initially establish a biotrophic interaction with their hosts that is succeeded at some point in time, for some sooner and for others later, by a necrotrophic phase. Importantly, extensive colonization of the apoplast is predominantly restricted to specialized microbes, including pathogens, with the ability to subvert host immunity using effector molecules ${ }^{5,25}$. As a consequence, the microbial densities encountered by phyllosphere-colonizing plant pathogens in the apoplast are generally much lower than in the episphere ${ }^{17,240}$. Consequently, the potential importance of antimicrobial effectors for the suppression of niche competitors in the apoplast might be limited. Nevertheless, mass spectrometry analyses on the apoplastic fluid obtained from tomato plants infected with the biotrophic fungal pathogen Cladosporium fulvum revealed that $10 \%$ (7 out of 70 ) of the identified in planta-secreted small proteins are predicted as structural homologs of known antimicrobial proteins ${ }^{241}$. Importantly, 
it needs to be noted that proteins with canonical effector characteristics, such as small size and high cysteine content, are likely to adopt tight folds that resemble confirmations as observed for toxins and defensins, which could make them prone to misclassification as AMPs. Nevertheless, the observation as made for $C$. fulvum could point towards the potential involvement of pathogen effector proteins in microbial competition in the leaf apoplast.

During necrotrophic infection stages, plant pathogens actively induce plant tissue necrosis, which is accompanied by the dissipation of host immune responses, which makes the plant tissue an attractive niche for opportunistic microbes. Hence, microbial competition for pathogenic microbes is likely to increase during the transition from biotrophy to necrotrophy. Consequently, plant pathogens with necrotrophic life stages can be anticipated to exploit antimicrobial effector proteins to protect their niche from the suite of new competitors that emerge once host immunity fades. Nevertheless, experimental evidence for this hypothesis has been lacking. However, our findings on the role of the $V$. dahliae effector VdAMP3 in the protection of microsclerotia formation in the decaying host phyllosphere underpin the relevance of the exploitation of antimicrobial effectors in necrotic host tissues (Chapter 6).

\section{Microbiome-manipulating effector proteins: specialists rather than generalists?}

A multitude of strategies, including proteomics, transcriptomics and comparative genomics, have proven to be successful for the identification of important plant pathogen effectors that are involved in the typical modulation of host physiology, with a particular focus on host immune responses ${ }^{242-244}$. Obviously, at present, many approaches that could potentially facilitate the identification of microbiomemanipulating effectors have remained unexplored. Recently, we proposed that transcriptional analysis of plant pathogens undergoing microbial competition in vitro might reveal the induction of effector genes potentially involved in microbial interactions ${ }^{78}$. However, transcriptome analyses following in vitro cultivation of $V$. dahliae in the presence of other microbes, or in the presence of the bacterial cell wall polymer peptidoglycan, did not reveal induction of any of the effector candidates that are predicted to share structural homology with known $\mathrm{AMPs}^{134}$. Instead, we discovered that $V d A v e 1, V d A M P 2$ and $V d A M P 3$ are expressed in a life stagedependent manner which does not require microbial exposure per se ${ }^{134}$ (Chapter 3 , Chapter 6). For instance, VdAve1 was not only shown to be highly expressed during $V$. dahliae host colonization, but also during in vitro cultivation in the absence of microbes. Additionally, VdAMP2 was shown to be induced upon in vitro cultivation of $V$. dahliae in sterile soil extract ${ }^{134}$, whereas $V d A M P 3$ is strongly induced during $V$. dahliae microsclerotia formation in planta as well as under sterile conditions in vitro. 
The continuous microbial exposure of plant pathogens in all the different niches that they colonize as part of their life cycle is likely to require a constant secretion of antimicrobials to promote niche protection or establishment. Hence, in retrospect, it is conceivable that more sophisticated factors than simply microbial exposure determine antimicrobial effector gene expression.

Arguably, distinct niches with divergent microbial communities and abiotic conditions require AMPs with specialized activities that are tailored for these specific environments. Consequently, like V. dahliae, other plant pathogens might tailor the expression of their microbiome-manipulating effectors according to their life stages and the corresponding niche that they aim to colonize at that stage. A factor that could require tight microbiome-manipulating effector gene expression is the potential impact of an AMP on host tissues. Previously, we suggested that effector proteins could be broadly classified into three groups: plant-targeting effectors, microbe-targeting effectors and multifunctional effectors targeting plants and microbes ${ }^{78}$. Arguably, effectors from the latter group exhibiting phytotoxic and antimicrobial activity would represent exquisite tools for necrotrophs or hemibiotrophs to simultaneously induce host cell death and to suppress microbial competitors. In contrast, however, the expression of such effectors during nonnecrotrophic life stages or by pathogens that do not exert necrotrophy could hamper host colonization as damage to host cells may betray attempted pathogen ingress. Transcriptional analyses revealed that VdAMP2 is expressed during $V$. dahliae soil colonization, but not during colonization of roots (Chapter 3 ). Intriguingly, when we attempted transient expression of VdAMP2 in N. benthamiania leaves, clear tissue necrosis was observed, suggesting phytotoxicity of the effector (Snelders and Thomma, unpublished data). Hence, $V$. dahliae is likely to repress VdAMP2 expression in the roots to limit host cell damage to not compromise colonization, which again supports the notion that antimicrobial effector proteins secreted by plant pathogens act as specialists in particular niches and do not serve as generalists that are being exploited throughout entire life cycles upon microbial exposure.

\section{Effector-mediated associations with microbial co-operators}

Studies of microbial interactions in the plant holobiont have predominantly addressed antagonism and competition. Nevertheless, plant-associated microbes also engage in commensalistic and mutualistic interactions with microbial co-inhabitants that colonize the outside as well as the inside of these tissues ${ }^{245,246}$. Fungal hyphae and their immediate surroundings, the so-called "hyphosphere" ${ }^{247}$, form a microhabitat that can be colonized by specialized microbial communities ${ }^{248-251}$. Thus far, the microbial communities surrounding the mycelial networks formed by arbuscular mycorrhizal fungi have predominantly been studied ${ }^{252}$. Intriguingly, analogous to the role of plant 
root exudates in the assembly of root microbiomes, multiple in vitro experiments provided evidence that (carbon-rich) fungal exudates can stimulate the growth of specific bacteria and impact microbial community structures $250,253,254$. Importantly, bacterial symbionts can fulfill beneficial activities for fungi and, for instance, have been implicated in the establishment of symbiosis between arbuscular mycorrhizal fungi and plants, as well as in the protection of soil-borne fungi from antifungal compounds ${ }^{255-258}$. Although evidence for intimate hyphosphere interactions between bacteria and fungi in the context of the plant holobiont is presently largely limited to arbuscular mycorrhizal fungi and their ectosymbionts, fungal plant pathogens can similarly be anticipated to establish intimate associations with microbial co-inhabitants. Fungal hyphae have been demonstrated to act as vectors that facilitate migration of bacteria ${ }^{259-262}$. Hence, fungal plant pathogens might recruit cooperative ectosymbiotic bacteria to migrate along their hyphae and to serve in the colonization of the various niches that are encountered during their life cycle. For instance, bacterial symbionts could aid in the suppression of antagonists, or might confer protection against antimicrobials secreted by plants hosts or microbial coinhabitants. Alternatively, bacterial symbionts might even contribute to direct host manipulation. Although not immediately addressing a bacterial ectosymbiont, the fungal plant pathogen Rhizopus microsporus, causal agent of Rice Seedling Blight, carries an endosymbiotic Burkholderia strain that is responsible for the synthesis of the phytotoxic metabolite Rhizoxin, which acts as a crucial virulence factor for rice colonization by the fungus ${ }^{245,263}$. Similarly, infection of several fungal plant pathogens by viruses was shown to promote their virulence 264,265 . Hence, the recruitment of microbial symbionts can extend the virulence potential of fungal pathogens. Possibly, effector proteins could play a role in such a recruitment. Although the initial attraction of bacteria to the hyphosphere might in part be based on exudates released by the fungus, effector proteins may aid in shaping the attracted community and could facilitate the accommodation of specific microbes. For instance, the secretion of antimicrobial effector proteins could enrich for beneficial symbionts that are tolerant to the AMP and therefore benefit from a competitive advantage over other microbes. Alternatively, effector proteins could aid in the establishment of physical interactions between plant pathogens and their symbionts.

\section{Implications and applications}

Ever since their first characterization as virulence factors, the study of effector proteins has been strongly focused on their importance for direct manipulation of host physiology, with a strong focus on host immune responses. However, over time, the emergence of next generation sequencing technologies has spurred the characterization of plant microbiomes, which greatly enhanced our understanding 
of their importance for plant health in turn. Our work describing effector-mediated microbiome manipulation by the soil-borne fungal pathogen $V$. dahliae has uncovered that the impact of effector proteins extends beyond the direct interactions between a pathogen and its host. As a consequence, in some cases, the field of effector biology might need to refocus its view on the role of effector proteins from binary plant-pathogen interactions to the more complex interactions in the plant holobiont. Importantly, such a new view also offers new perspectives for disease control.

Since the advent of fungi on earth preceded land plant evolution, a substantial part of the microbiome-manipulating effector genes can be speculated to be the remnants of genes that initially aided in microbial competition in the soil. Nevertheless, the functional characterization of the $V$. dahliae effector VdAve1, which was horizontally acquired from plants, indicates that plant pathogens also evolved effector proteins more recently to impact host microbiomes. Importantly, as evident from the recognition of $\mathrm{VdAve} 1$ by its immune receptor $\mathrm{Ve} 1^{161}$, plants in turn evolved to recognize such microbiome-manipulating effectors. As a consequence, plant pathogens may mutate or lose their microbiome-manipulating effectors to evade recognition, leading to pathogen races with different suites of microbe-targeting effectors. Accordingly, the identification and characterization of microbiome-manipulating effectors could aid in the development of more targeted biocontrol strategies. A possibility for more effective use of microbial biocontrol agents would be to base their selection on the genotype of a plant pathogen, for instance by selecting antagonists that are insensitive for the activity of a particular effector. Conversely, in case a resistance gene has been described to recognize a microbiome-manipulating effector protein, the application of a strong antagonistic biocontrol agent that is sensitive for the effector activity could be considered. In this manner, a strong selection pressure might be exerted on the retainment of that particular effector gene, which in turn could contribute to enhanced durability of a resistance gene. Additionally, the identification of microbial symbionts, and the characterization of the mechanisms underlying the interaction with a plant pathogen, could open up new possibilities for pathogen control. One possibility for improved biological control of plant pathogens would be to select for, or to even engineer, biocontrol agents that can be applied in agricultural settings to act as a decoy to "trap" plant pathogens in destructive interactions that impair their virulence.

Intermicrobial interactions occur in a wide diversity of niches as they are found on our planet. Molecules secreted by microbes play pivotal roles in these interactions as these can be exploited to exert positive or negative impact on microbial co-inhabitants. An extensive array of microbially-secreted molecules has been implicated in microbial competition, including secondary metabolites, hydrolytic enzymes and AMPs such as defensins and toxins. Some Gram-negative bacteria even employ specialized 
secretion machinery, such as the type VI secretion system, to directly deliver antimicrobial effectors into neighboring microbes ${ }^{90}$. Antimicrobials secreted by microbes represent a valuable resource of molecules that can be applied for various purposes, including food preservation or treatment of infections in animals and in humans ${ }^{129,266}$. For instance, soil-dwelling microbes, predominantly Actinobacteria, are a primary source for clinically used antibiotics ${ }^{128,129}$. Bacterial antibiotic resistance is a continuously increasing problem. Based on our discovery that the effector protein repertoire of $V$. dahliae comprises antimicrobials intended for microbial competition, we propose that plant pathogen effectors represent a new category of microbially-secreted molecules that can be mined for novel antimicrobials, such as VdAve1 (Chapter 3-5). It needs to be noted that the antimicrobial in plantasecreted $V$. dahliae effectors described in this thesis, VdAve1 and VdAMP3, display resemblance with antimicrobials categorized as hydrolytic enzymes or defensins. At the same time, both proteins satisfy the canonical characteristics as typically used to categorize pathogen effectors, namely being small cysteine-rich and secreted in planta to promote colonization. Hence, the distinction between effector proteins and known antimicrobials from non-pathogenic microbes might be blurred in particular cases, which supports our suggestion to define effectors as microbiallysecreted molecules that act in niche colonization, and not in host colonization per se (Chapters $1 \& 2)^{25}$. A potential advantage of mining effector repertoires for antimicrobials is the fact that plant pathogens occupy different niches when compared with most producers of antimicrobial compounds that have been identified thus far. As a consequence, plant pathogens encounter different microbial communities under different conditions. Hence, depending on the niche in which they are secreted, antimicrobial effector proteins of pathogenic fungi might have evolved different activity spectra than antimicrobials secreted by microbes from other niches. Consequently, microbiome-manipulating effectors could complement the activities of presently described antimicrobials.

In conclusion, our findings as described in this thesis establish a role for plant pathogen effector proteins in microbiome manipulation, offer novel perspectives for plant disease control, and may facilitate the identification and development of novel antibiotics. 



\section{References}

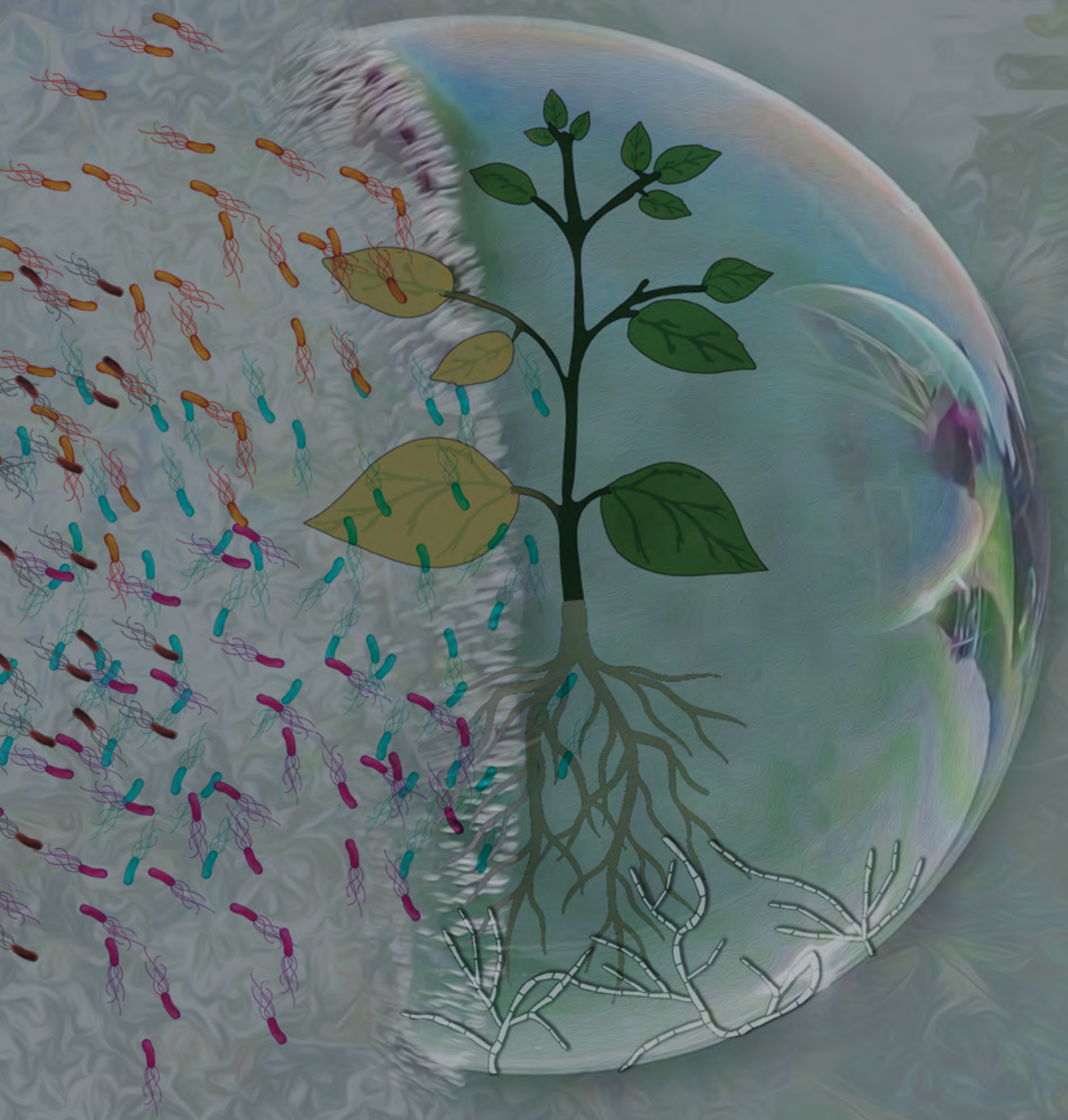


1. Dodds, P. N. \& Rathjen, J. P. Plant immunity: towards an integrated view of plant-pathogen interactions. Nat. Rev. Genet. 11, 539-548 (2010).

2. Jones, J. D. G. \& Dangl, J. L. The plant immune system. Nature 444, 323-329 (2006).

3. Pritchard, L. \& Birch, P. R. The zigzag model of plant-microbe interactions: is it time to move on? Mol. Plant Pathol. 15, 865-870 (2014).

4. Thomma, B. P. H. J., Nürnberger, T. \& Joosten, M. H. A. J. Of PAMPs and effectors: the blurred PTI-ETI dichotomy. Plant Cell 23, 4-15 (2011).

5. Cook, D. E., Mesarich, C. H. \& Thomma, B. P. H. J. Understanding plant immunity as a surveillance system to detect invasion. Annu. Rev. Phytopathol. 53, 541-563 (2015).

6. Marchesi, J. R. \& Ravel, J. The vocabulary of microbiome research: a proposal. (2015).

7. Vorholt, J. A. Microbial life in the phyllosphere. Nat. Rev. Microbiol. 10, 828-840 (2012).

8. Innerebner, G., Knief, C. \& Vorholt, J. A. Protection of Arabidopsis thaliana against leaf-pathogenic Pseudomonas syringae by Sphingomonas strains in a controlled model system. Appl. Environ. Microbiol. 77, 3202-3210 (2011).

9. Dimkpa, C., Weinand, T. \& Asch, F. Plant-rhizobacteria interactions alleviate abiotic stress conditions. Plant. Cell Environ. 32, 1682-1694 (2009).

10. Berendsen, R. L., Pieterse, C. M. J. \& Bakker, P. A. H. M. The rhizosphere microbiome and plant health. Trends Plant Sci. 17, 478-486 (2012).

11. Fitzpatrick, C. R. et al. Assembly and ecological function of the root microbiome across angiosperm plant species. Proc. Natl. Acad. Sci. 115, E1157-E1165 (2018).

12. Doornbos, R. F., van Loon, L. C. \& Bakker, P. A. H. M. Impact of root exudates and plant defense signaling on bacterial communities in the rhizosphere. A review. Agron. Sustain. Dev. 32, 227-243 (2012).

13. Bais, H. P., Weir, T. L., Perry, L. G., Gilroy, S. \& Vivanco, J. M. The role of root exudates in rhizosphere interactions with plants and other organisms. Annu. Rev. Plant Biol. 57, 233-266 (2006).

14. Turner, T. R., James, E. K. \& Poole, P. S. The plant microbiome. Genome Biol. 14, 209 (2013).

15. Berendsen, R. L. et al. Disease-induced assemblage of a plant-beneficial bacterial consortium. ISME J. 12, 1496-1507 (2018).

16. Koprivova, A. et al. Root-specific camalexin biosynthesis controls the plant growth-promoting effects of multiple bacterial strains. Proc. Natl. Acad. Sci. 116, 15735-15744 (2019).

17. Huang, A. C. et al. A specialized metabolic network selectively modulates Arabidopsis root microbiota. Science. 364, eaau6389 (2019).

18. Castrillo, G. et al. Root microbiota drive direct integration of phosphate stress and immunity. Nature 543, 513-518 (2017).

19. Mendes, R. et al. Deciphering the rhizosphere microbiome for disease-suppressive bacteria. Science. 332, 1097-1100 (2011).

20. Rudrappa, T., Czymmek, K. J., Paré, P. W. \& Bais, H. P. Root-secreted malic acid recruits beneficial soil bacteria. Plant Physiol. 148, 1547-1556 (2008).

21. Carrión, V. J. et al. Pathogen-induced activation of disease-suppressive functions in the endophytic root microbiome. Science. 366, 606-612 (2019).

22. Compant, S., Duffy, B., Nowak, J., Clément, C. \& Barka, E. A. Use of plant growth-promoting bacteria for biocontrol of plant diseases: principles, mechanisms of action, and future prospects. Appl. Environ. Microbiol. 71, 4951-4959 (2005).

23. Pieterse, C. M. J. et al. Induced systemic resistance by beneficial microbes. Annu. Rev. Phytopathol. 52, 347-375 (2014).

24. Dini-Andreote, F. Endophytes: The Second Layer of Plant Defense. Trends Plant Sci. 25, 319-322 (2020).

25. Rovenich, H., Boshoven, J. C. \& Thomma, B. P. H. J. Filamentous pathogen effector functions: of pathogens, hosts and microbiomes. Curr. Opin. Plant Biol. 20, 96-103 (2014).

26. Rodriguez-Moreno, L., Ebert, M. K., Bolton, M. D. \& Thomma, B. P. H. J. Tools of the crook-infection strategies of fungal plant pathogens. Plant J. 93, 664-674 (2018). 
27. Weiberg, A. et al. Fungal small RNAs suppress plant immunity by hijacking host RNA interference pathways. Science. 342, 118-123 (2013).

28. Wolpert, T. J., Dunkle, L. D. \& Ciuffetti, L. M. Host-selective toxins and avirulence determinants: what's in a name? Annu. Rev. Phytopathol. 40, 251-285 (2002).

29. Cox, K. L. et al. TAL effector driven induction of a SWEET gene confers susceptibility to bacterial blight of cotton. Nat. Commun. 8, 1-14 (2017).

30. De Jonge, R. et al. Conserved fungal LysM effector Ecp6 prevents chitin-triggered immunity in plants. Science. 329, 953-955 (2010).

31. Maillet, F. et al. Fungal lipochitooligosaccharide symbiotic signals in arbuscular mycorrhiza. Nature 469, 58-63 (2011).

32. Zeng, T. et al. A lysin motif effector subverts chitin-triggered immunity to facilitate arbuscular mycorrhizal symbiosis. New Phytol. 225, 448-460 (2020).

33. Wawra, S. et al. The fungal-specific $\beta$-glucan-binding lectin FGB1 alters cell-wall composition and suppresses glucan-triggered immunity in plants. Nat. Commun. 7, 1-11 (2016).

34. De Jonge, R. \& Thomma, B. P. H. J. Fungal LysM effectors: extinguishers of host immunity? Trends Microbiol. 17, 151-157 (2009).

35. Sanchez-Vallet, A., Mesters, J. R. \& Thomma, B. P. H. J. The battle for chitin recognition in plantmicrobe interactions. FEMS Microbiol. Rev. 39, 171-183 (2015).

36. Kombrink, A. et al. Verticillium dahliae LysM effectors differentially contribute to virulence on plant hosts. Mol. Plant Pathol. 18, 596-608 (2017).

37. Marshall, R. et al. Analysis of two in planta expressed LysM effector homologs from the fungus Mycosphaerella graminicola reveals novel functional properties and varying contributions to virulence on wheat. Plant Physiol. 156, 756-769 (2011).

38. Gijzen, M. \& Nürnberger, T. Nep1-like proteins from plant pathogens: recruitment and diversification of the NPP1 domain across taxa. Phytochemistry 67, 1800-1807 (2006).

39. Pemberton, C. L. \& Salmond, G. P. C. The Nep1-like proteins-a growing family of microbial elicitors of plant necrosis. Mol. Plant Pathol. 5, 353-359 (2004).

40. Seidl, M. F. \& Van den Ackerveken, G. Activity and phylogenetics of the broadly occurring family of microbial Nep1-like proteins. Annu. Rev. Phytopathol. 57, 367-386 (2019).

41. Levy, A. et al. Genomic features of bacterial adaptation to plants. Nat. Genet. 50, 138-150 (2018).

42. Kettles, G. J. et al. Characterization of an antimicrobial and phytotoxic ribonuclease secreted by the fungal wheat pathogen Zymoseptoria tritici. New Phytol. 217, 320-331 (2018).

43. Kombrink, A. \& Thomma, B. P. H. J. LysM effectors: secreted proteins supporting fungal life. PLoS Pathog. 9, (2013).

44. Inderbitzin, P. et al. Phylogenetics and taxonomy of the fungal vascular wilt pathogen Verticillium, with the descriptions of five new species. PLoS One 6, (2011).

45. Qin, Q.-M., Vallad, G. E. \& Subbarao, K. V. Characterization of Verticillium dahliae and V. tricorpus isolates from lettuce and artichoke. Plant Dis. 92, 69-77 (2008).

46. Seidl, M. F. et al. The genome of the saprophytic fungus Verticillium tricorpus reveals a complex effector repertoire resembling that of its pathogenic relatives. Mol. Plant-Microbe Interact. 28, 362373 (2015).

47. Klosterman, S. J., Atallah, Z. K., Vallad, G. E. \& Subbarao, K. V. Diversity, pathogenicity, and management of Verticillium species. Annu. Rev. Phytopathol. 47, 39-62 (2009).

48. Fradin, E. F. \& Thomma, B. P. H. J. Physiology and molecular aspects of Verticillium wilt diseases caused by V. dahliae and V. albo-atrum. Mol. Plant Pathol. 7, 71-86 (2006).

49. Depotter, J. R. L. et al. Verticillium longisporum, the invisible threat to oilseed rape and other brassicaceous plant hosts. Mol. Plant Pathol. 17, 1004-1016 (2016).

50. Flajsman, M. et al. Identification of novel virulence-associated proteins secreted to xylem by Verticillium nonalfalfae during colonization of hop plants. Mol. Plant-Microbe Interact. 29, 362-373 (2016). 
51. Inderbitzin, P., Davis, R. M., Bostock, R. M. \& Subbarao, K. V. The ascomycete Verticillium longisporum is a hybrid and a plant pathogen with an expanded host range. PLoS One 6, (2011).

52. Inderbitzin, P. \& Subbarao, K. V. Verticillium systematics and evolution: how confusion impedes Verticillium wilt management and how to resolve it. Phytopathology 104, 564-574 (2014).

53. Wilhelm, S. Longevity of the Verticillium wilt fungus in the laboratory and field. Phytopathology 45, 180-181 (1955).

54. Olsson, S. \& Nordbring-Hertz, B. Microsclerotial germination of Verticillium dahliae as affected by rape rhizosphere. FEMS Microbiol. Ecol. 1, 293-299 (1985).

55. Mol, L. \& Van Riessen, H. W. Effect of plant roots on the germination of microsclerotia of Verticillum dahliae. Eur. J. plant Pathol. 101, 673-678 (1995).

56. Huisman, O. C. \& Ashworth Jr, L. J. Influence of crop rotation on survival of Verticillium albo-atrum in soils. Phytopathology 66, 978-981 (1976).

57. Diwan, N., Fluhr, R., Eshed, Y., Zamir, D. \& Tanksley, S. D. Mapping of Ve in tomato: a gene conferring resistance to the broad-spectrum pathogen, Verticillium dahliae race 1. Theor. Appl. Genet. 98, 315319 (1999).

58. Schaible, L., Cannon, O. S. \& Waddoups, V. Inheritance of resistance to Verticillium wilt in a tomato cross. Phytopathology 41, (1951).

59. Fradin, E. F. et al. Genetic dissection of Verticillium wilt resistance mediated by tomato Ve1. Plant Physiol. 150, 320-332 (2009).

60. Alexander, L. J. Susceptibility of certain Verticillium-resistant tomato varieties to an Ohio isolate of the pathogen. Phytopathology 52, 998-1000 (1962).

61. de Jonge, R. et al. Tomato immune receptor Ve1 recognizes effector of multiple fungal pathogens uncovered by genome and RNA sequencing. Proc. Natl. Acad. Sci. 109, 5110-5115 (2012).

62. de Jonge, R. et al. Extensive chromosomal reshuffling drives evolution of virulence in an asexual pathogen. Genome Res. 23, 1271-1282 (2013).

63. Song, Y. et al. Broad taxonomic characterization of Verticillium wilt resistance genes reveals an ancient origin of the tomato Ve1 immune receptor. Mol. Plant Pathol. 18, 195-209 (2017).

64. Dobinson, K. F., Tenuta, G. K. \& Lazarovits, G. Occurrence of race 2 of Verticillium dahliae in processing tomato fields in southwestern Ontario. Can. J. Plant Pathol. 18, 55-58 (1996).

65. Usami, T. et al. Race 2 of Verticillium dahliae infecting tomato in Japan can be split into two races with differential pathogenicity on resistant rootstocks. Plant Pathol. 66, 230-238 (2017).

66. Chavarro-Carrero, E. et al. Comparative genomics of Verticillium dahliae isolates reveals the in planta-secreted effector protein recognized in V2 tomato plants. BioRxiv (2020).

67. Deketelaere, S., Tyvaert, L., França, S. C. \& Höfte, M. Desirable traits of a good biocontrol agent against Verticillium wilt. Front. Microbiol. 8, 1186 (2017).

68. Mercado-Blanco, J., Rodrıguez-Jurado, D., Hervás, A. \& Jiménez-Dıaz, R. M. Suppression of Verticillium wilt in olive planting stocks by root-associated fluorescent Pseudomonas spp. Biol. Control 30, 474486 (2004).

69. Antonopoulos, D. F., Tjamos, S. E., Antoniou, P. P., Rafeletos, P. \& Tjamos, E. C. Effect of Paenibacillus alvei, strain $\mathrm{K} 165$, on the germination of Verticillium dahliae microsclerotia in planta. Biol. Control 46, 166-170 (2008).

70. Tyvaert, L., França, S. C., Debode, J. \& Höfte, M. The endophyte Verticillium Vt305 protects cauliflower against Verticillium wilt. J. Appl. Microbiol. 116, 1563-1571 (2014).

71. Fatima, U. \& Senthil-Kumar, M. Plant and pathogen nutrient acquisition strategies. Front. Plant Sci. 6, 750 (2015).

72. Wang, M. et al. Bidirectional cross-kingdom RNAi and fungal uptake of external RNAs confer plant protection. Nat. plants 2, 1-10 (2016).

73. Liu, Z. et al. SnTox1, a Parastagonospora nodorum necrotrophic effector, is a dual-function protein that facilitates infection while protecting from wheat-produced chitinases. New Phytol. 211, 10521064 (2016). 
74. Stergiopoulos, I. \& de Wit, P. J. G. M. Fungal effector proteins. Annu. Rev. Phytopathol. 47, 233-263 (2009).

75. Huttenhower, C. et al. Structure, function and diversity of the healthy human microbiome. Nature 486, 207 (2012).

76. Bulgarelli, D. et al. Revealing structure and assembly cues for Arabidopsis root-inhabiting bacterial microbiota. Nature 488, 91-95 (2012).

77. Lundberg, D. S. et al. Defining the core Arabidopsis thaliana root microbiome. Nature 488, 86-90 (2012).

78. Snelders, N. C., Kettles, G. J., Rudd, J. J. \& Thomma, B. P. H. J. Plant pathogen effector proteins as manipulators of host microbiomes? Mol. Plant Pathol. 19, 257 (2018).

79. Ficarra, F. A., Grandellis, C., Garavaglia, B. S., Gottig, N. \& Ottado, J. Bacterial and plant natriuretic peptides improve plant defence responses against pathogens. Mol. Plant Pathol. 19, 801-811 (2018).

80. Gehring, C. A. \& Irving, H. R. Natriuretic peptides-a class of heterologous molecules in plants. Int. J. Biochem. Cell Biol. 35, 1318-1322 (2003).

81. Faino, L., de Jonge, R. \& Thomma, B. P. H. J. The transcriptome of Verticillium dahliae infected Nicotiana benthamiana determined by deep RNA sequencing. Plant Signal. Behav. 7, 1065-1069 (2012).

82. Ingham, A. B. \& Moore, R. J. Recombinant production of antimicrobial peptides in heterologous microbial systems. Biotechnol. Appl. Biochem. 47, 1-9 (2007).

83. Takeuchi, M., Hamana, K. \& Hiraishi, A. Proposal of the genus Sphingomonas sensu stricto and three new genera, Sphingobium, Novosphingobium and Sphingopyxis, on the basis of phylogenetic and chemotaxonomic analyses. Int. J. Syst. Evol. Microbiol. 51, 1405-1417 (2001).

84. Aylward, F. O. et al. Comparison of 26 sphingomonad genomes reveals diverse environmental adaptations and biodegradative capabilities. Appl. Environ. Microbiol. 79, 3724-3733 (2013).

85. Bai, Y. et al. Functional overlap of the Arabidopsis leaf and root microbiota. Nature 528, 364-369 (2015).

86. Faino, L. et al. Single-molecule real-time sequencing combined with optical mapping yields completely finished fungal genome. MBio 6, (2015).

87. Depotter, J. et al. Homogenization of sub-genome secretome gene expression patterns in the allodiploid fungus Verticillium longisporum. BioRxiv 341636 (2018).

88. Gibriel, H., Li, J., Zhu, L., Seidl, M. F. \& Thomma, B. P. H. J. Verticillium dahliae strains that infect the same host plant display highly divergent effector catalogs. bioRxiv 528729 (2019).

89. Dal Peraro, M. \& Van Der Goot, F. G. Pore-forming toxins: ancient, but never really out of fashion. Nat. Rev. Microbiol. 14, 77-92 (2016).

90. Coulthurst, S. The Type VI secretion system: a versatile bacterial weapon. Microbiology 165, 503-515 (2019).

91. Zhao, W., Caro, F., Robins, W. \& Mekalanos, J. J. Antagonism toward the intestinal microbiota and its effect on Vibrio cholerae virulence. Science. 359, 210-213 (2018).

92. Alfano, J. R. \& Collmer, A. Type III secretion system effector proteins: double agents in bacterial disease and plant defense. Annu. Rev. Phytopathol. 42, 385-414 (2004).

93. Xiong, D. et al. Deep mRNA sequencing reveals stage-specific transcriptome alterations during microsclerotia development in the smoke tree vascular wilt pathogen, Verticillium dahliae. BMC Genomics 15, 324 (2014).

94. Zhang, Z. et al. Optimized agroinfiltration and virus-induced gene silencing to study Ve1-mediated Verticillium resistance in tobacco. Mol. plant-microbe Interact. 26, 182-190 (2013).

95. Frandsen, R. J. N., Andersson, J. A., Kristensen, M. B. \& Giese, H. Efficient four fragment cloning for the construction of vectors for targeted gene replacement in filamentous fungi. BMC Mol. Biol. 9, 70 (2008).

96. Santhanam, P. Random insertional mutagenesis in fungal genomes to identify virulence factors. in Plant Fungal Pathogens 509-517 (Springer, 2012). 
97. Bozzola, J. J. Conventional specimen preparation techniques for scanning electron microscopy of biological specimens. in Electron Microscopy 133-150 (Springer, 2014).

98. Callahan, B. J., Sankaran, K., Fukuyama, J. A., McMurdie, P. J. \& Holmes, S. P. Bioconductor workflow for microbiome data analysis: from raw reads to community analyses. F1000Research 5, (2016).

99. Callahan, B. J. et al. DADA2: high-resolution sample inference from Illumina amplicon data. Nat. Methods 13, 581-583 (2016).

100. McMurdie, P. J. \& Holmes, S. phyloseq: an R package for reproducible interactive analysis and graphics of microbiome census data. PLoS One 8, e61217 (2013).

101. Love, M. I., Huber, W. \& Anders, S. Moderated estimation of fold change and dispersion for RNA-seq data with DESeq2. Genome Biol. 15, 550 (2014).

102. Depotter, J. R. L., Thomma, B. P. H. J. \& Wood, T. A. Measuring the impact of Verticillium longisporum on oilseed rape (Brassica napus) yield in field trials in the United Kingdom. Eur. J. plant Pathol. 153, 321-326 (2019).

103. Kelley, L. A., Mezulis, S., Yates, C. M., Wass, M. N. \& Sternberg, M. J. E. The Phyre2 web portal for protein modeling, prediction and analysis. Nat. Protoc. 10, 845 (2015).

104. Schneider, J. J., Unholzer, A., Schaller, M., Schäfer-Korting, M. \& Korting, H. C. Human defensins. J. Mol. Med. 83, 587-595 (2005).

105. Reichling, J. Plant-microbe interactions and secondary metabolites with antibacterial, antifungal and antiviral properties. Annu. Plant Rev. online 214-347 (2018).

106. Ganz, T. Defensins: antimicrobial peptides of innate immunity. Nat. Rev. Immunol. 3, 710-720 (2003).

107. Sato, H. \& Feix, J. B. Peptide-membrane interactions and mechanisms of membrane destruction by amphipathic a-helical antimicrobial peptides. Biochim. Biophys. Acta (BBA)-Biomembranes 1758, 1245-1256 (2006).

108. Epand, R. M. \& Vogel, H. J. Diversity of antimicrobial peptides and their mechanisms of action. Biochim. Biophys. Acta (BBA)-Biomembranes 1462, 11-28 (1999).

109. Nguyen, L. T., Haney, E. F. \& Vogel, H. J. The expanding scope of antimicrobial peptide structures and their modes of action. Trends Biotechnol. 55, 27-55 (2011).

110. Yeaman, M. R. \& Yount, N. Y. Mechanisms of antimicrobial peptide action and resistance. Pharmacol. Rev. 55, 27-55 (2003).

111. Shafee, T. \& Anderson, M. A. A quantitative map of protein sequence space for the cis-defensin superfamily. Bioinformatics 35, 743-752 (2019).

112. Zhu, S. Discovery of six families of fungal defensin-like peptides provides insights into origin and evolution of the CSaß defensins. Mol. Immunol. 45, 828-838 (2008).

113. Aerts, A. M., François, I., Cammue, B. P. A. \& Thevissen, K. The mode of antifungal action of plant, insect and human defensins. Cell. Mol. life Sci. 65, 2069-2079 (2008).

114. Terras, F. R. et al. Analysis of two novel classes of plant antifungal proteins from radish (Raphanus sativus L.) seeds. J. Biol. Chem. 267, 15301-15309 (1992).

115. Terras, F. R. et al. Small cysteine-rich antifungal proteins from radish: their role in host defense. Plant Cell 7, 573-588 (1995).

116. Thomma, B. P. H. J., Cammue, B. P. \& Thevissen, K. Plant defensins. Planta 216, 193-202 (2002).

117. Terras, F. R. G., Penninckx, I. A. M. A., Goderis, I. J. \& Broekaert, W. F. Evidence that the role of plant defensins in radish defense responses is independent of salicylic acid. Planta 206, 117-124 (1998).

118. Berrocal-Lobo, M. et al. Snakin-2, an antimicrobial peptide from potato whose gene is locally induced by wounding and responds to pathogen infection. Plant Physiol. 128, 951-961 (2002).

119. Do, H. M., Lee, S. C., Jung, H. W., Sohn, K. H. \& Hwang, B. K. Differential expression and in situ localization of a pepper defensin (CADEF1) gene in response to pathogen infection, abiotic elicitors and environmental stresses in Capsicum annuum. Plant Sci. 166, 1297-1305 (2004).

120. Penninckx, I. A. M. A., Thomma, B. P. H. J., Buchala, A., Métraux, J.-P. \& Broekaert, W. F. Concomitant activation of jasmonate and ethylene response pathways is required for induction of a plant defensin gene in Arabidopsis. Plant Cell 10, 2103-2113 (1998). 
121. Thomma, B. P. H. J. et al. Separate jasmonate-dependent and salicylate-dependent defenseresponse pathways in Arabidopsis are essential for resistance to distinct microbial pathogens. Proc. Natl. Acad. Sci. 95, 15107-15111 (1998).

122. González-Lamothe, R. et al. Plant antimicrobial agents and their effects on plant and human pathogens. Int. J. Mol. Sci. 10, 3400-3419 (2009).

123. Ahuja, I., Kissen, R. \& Bones, A. M. Phytoalexins in defense against pathogens. Trends Plant Sci. 17, 73-90 (2012).

124. Hibbing, M. E., Fuqua, C., Parsek, M. R. \& Peterson, S. B. Bacterial competition: surviving and thriving in the microbial jungle. Nat. Rev. Microbiol. 8, 15-25 (2010).

125. Ghoul, M. \& Mitri, S. The ecology and evolution of microbial competition. Trends Microbiol. 24, 833845 (2016).

126. García-Bayona, L. \& Comstock, L. E. Bacterial antagonism in host-associated microbial communities. Science. 361, eaat2456 (2018).

127. O'Brien, J. \& Wright, G. D. An ecological perspective of microbial secondary metabolism. Curr. Opin. Biotechnol. 22, 552-558 (2011).

128. Baltz, R. H. Renaissance in antibacterial discovery from actinomycetes. Curr. Opin. Pharmacol. 8, 557563 (2008).

129. Van der Meij, A., Worsley, S. F., Hutchings, M. I. \& van Wezel, G. P. Chemical ecology of antibiotic production by actinomycetes. FEMS Microbiol. Rev. 41, 392-416 (2017).

130. Toruño, T. Y., Stergiopoulos, I. \& Coaker, G. Plant-pathogen effectors: cellular probes interfering with plant defenses in spatial and temporal manners. Annu. Rev. Phytopathol. 54, 419-441 (2016).

131. Varden, F. A., De la Concepcion, J. C., Maidment, J. H. R. \& Banfield, M. J. Taking the stage: effectors in the spotlight. Curr. Opin. Plant Biol. 38, 25-33 (2017).

132. Lee, H.-J., Jo, E.-J., Kim, N.-H., Chae, Y. \& Lee, S.-W. Disease responses of tomato pure lines against Ralstonia solanacearum strains from Korea and susceptibility at high temperature. Res. Plant Dis. 17, 326-333 (2011).

133. Song, Y. et al. Tomato immune receptor Ve1 recognizes surface-exposed co-localized $\mathrm{N}$-and C-termini of Verticillium dahliae effector Ave1. BioRxiv 103473 (2017).

134. Snelders, N. C. et al. Microbiome manipulation by a soil-borne fungal plant pathogen using effector proteins. Nature Plants (2020) in press.

135. Ruzvidzo, O., Donaldson, L., Valentine, A. \& Gehring, C. The Arabidopsis thaliana natriuretic peptide AtPNP-A is a systemic regulator of leaf dark respiration and signals via the phloem. J. Plant Physiol. 168, 1710-1714 (2011).

136. Marston, A. L., Thomaides, H. B., Edwards, D. H., Sharpe, M. E. \& Errington, J. Polar localization of the MinD protein of Bacillus subtilis and its role in selection of the mid-cell division site. Genes Dev. 12, 3419-3430 (1998).

137. Szeto, T. H., Rowland, S. L., Habrukowich, C. L. \& King, G. F. The MinD membrane targeting sequence is a transplantable lipid-binding helix. J. Biol. Chem. 278, 40050-40056 (2003).

138. Strahl, H. \& Hamoen, L. W. Membrane potential is important for bacterial cell division. Proc. Natl. Acad. Sci. 107, 12281-12286 (2010).

139. Müller, A. et al. Daptomycin inhibits cell envelope synthesis by interfering with fluid membrane microdomains. Proc. Natl. Acad. Sci. 113, E7077-E7086 (2016).

140. Saeloh, D. et al. The novel antibiotic rhodomyrtone traps membrane proteins in vesicles with increased fluidity. PLoS Pathog. 14, e1006876 (2018).

141. Li, J. Identification of host-specific effectors mediating pathogenicity of the vascular wilt pathogen Verticillium dahliae. (Wageningen university \& Research, 2019).

142. García, J.-R. C. et al. Human $\beta$-defensin 4: a novel inducible peptide with a specific salt-sensitive spectrum of antimicrobial activity. FASEB J. 15, 1819-1821 (2001).

143. Mukherjee, S. et al. Antibacterial membrane attack by a pore-forming intestinal C-type lectin. Nature 505, 103-107 (2014). 
144. Helmann, J. D. Bacillus subtilis extracytoplasmic function (ECF) sigma factors and defense of the cell envelope. Curr. Opin. Microbiol. 30, 122-132 (2016).

145. Ho, T. D., Hastie, J. L., Intile, P. J. \& Ellermeier, C. D. The Bacillus subtilis extracytoplasmic function $\sigma$ factor $\sigma \mathrm{V}$ is induced by lysozyme and provides resistance to lysozyme. J. Bacteriol. 193, 6215-6222 (2011).

146. Guariglia-Oropeza, V. \& Helmann, J. D. Bacillus subtilis $\sigma$ V confers lysozyme resistance by activation of two cell wall modification pathways, peptidoglycan O-acetylation and D-alanylation of teichoic acids. J. Bacteriol. 193, 6223-6232 (2011).

147. Herbert, S. et al. Molecular basis of resistance to muramidase and cationic antimicrobial peptide activity of lysozyme in staphylococci. PLoS Pathog. 3, (2007).

148. Düring, K., Porsch, P., Mahn, A., Brinkmann, O. \& Gieffers, W. The non-enzymatic microbicidal activity of lysozymes. FEBS Lett. 449, 93-100 (1999).

149. Ibrahim, H. R., Matsuzaki, T. \& Aoki, T. Genetic evidence that antibacterial activity of lysozyme is independent of its catalytic function. FEBS Lett. 506, 27-32 (2001).

150. Bera, A., Herbert, S., Jakob, A., Vollmer, W. \& Götz, F. Why are pathogenic staphylococci so lysozyme resistant? The peptidoglycan O-acetyltransferase OatA is the major determinant for lysozyme resistance of Staphylococcus aureus. Mol. Microbiol. 55, 778-787 (2005).

151. Neuhaus, F. C. \& Baddiley, J. A continuum of anionic charge: structures and functions of D-alanylteichoic acids in gram-positive bacteria. Microbiol. Mol. Biol. Rev. 67, 686-723 (2003).

152. Campbell, J. et al. Synthetic lethal compound combinations reveal a fundamental connection between wall teichoic acid and peptidoglycan biosyntheses in Staphylococcus aureus. ACS Chem. Biol. 6, 106-116 (2011).

153. Reichmann, N. T. \& Gründling, A. Location, synthesis and function of glycolipids and polyglycerolphosphate lipoteichoic acid in Gram-positive bacteria of the phylum Firmicutes. FEMS Microbiol. Lett. 319, 97-105 (2011).

154. Brown, S. et al. Methicillin resistance in Staphylococcus aureus requires glycosylated wall teichoic acids. Proc. Natl. Acad. Sci. 109, 18909-18914 (2012).

155. Kovács, $M$. et al. A functional dlt operon, encoding proteins required for incorporation of $d$-alanine in teichoic acids in gram-positive bacteria, confers resistance to cationic antimicrobial peptides in Streptococcus pneumoniae. J. Bacteriol. 188, 5797-5805 (2006).

156. Kristian, S. A. et al. D-alanylation of teichoic acids promotes group a Streptococcus antimicrobial peptide resistance, neutrophil survival, and epithelial cell invasion. J. Bacteriol. 187, 6719-6725 (2005).

157. Peschel, A. et al. Inactivation of the dlt operon in Staphylococcus aureus confers sensitivity to defensins, protegrins, and other antimicrobial peptides. J. Biol. Chem. 274, 8405-8410 (1999).

158. Van Opijnen, T., Bodi, K. L. \& Camilli, A. Tn-seq: high-throughput parallel sequencing for fitness and genetic interaction studies in microorganisms. Nat. Methods 6, 767 (2009).

159. Wu, S.-C. et al. Functional production and characterization of a fibrin-specific single-chain antibody fragment from Bacillus subtilis: effects of molecular chaperones and a wall-bound protease on antibody fragment production. Appl. Environ. Microbiol. 68, 3261-3269 (2002).

160. Kobayashi, K. et al. Essential Bacillus subtilis genes. Proc. Natl. Acad. Sci. 100, 4678-4683 (2003).

161. D'Elia, M. A., Henderson, J. A., Beveridge, T. J., Heinrichs, D. E. \& Brown, E. D. The N-acetylmannosamine transferase catalyzes the first committed step of teichoic acid assembly in Bacillus subtilis and Staphylococcus aureus. J. Bacteriol. 191, 4030-4034 (2009).

162. Brown, S., Santa Maria Jr, J. P. \& Walker, S. Wall teichoic acids of gram-positive bacteria. Annu. Rev. Microbiol. 67, 313-336 (2013).

163. Bera, A. et al. Influence of wall teichoic acid on lysozyme resistance in Staphylococcus aureus. J. Bacteriol. 189, 280-283 (2007).

164. Yamaguchi, H., Furuhata, K., Fukushima, T., Yamamoto, H. \& Sekiguchi, J. Characterization of a new Bacillus subtilis peptidoglycan hydrolase gene, yvcE (named cwlO), and the enzymatic properties of its encoded protein. J. Biosci. Bioeng. 98, 174-181 (2004). 
165. Margot, P., Wahlen, M., Gholamhuseinian, A., Piggot, P. \& Karamata, D. The lytE gene of Bacillus subtilis 168 encodes a cell wall hydrolase. J. Bacteriol. 180, 749-752 (1998).

166. Davies, R. C., Neuberger, A. \& Wilson, B. M. The dependence of lysozyme activity on pH and ionic strength. Biochim. Biophys. Acta (BBA)-Enzymology 178, 294-305 (1969).

167. van Opijnen, T., Lazinski, D. W. \& Camilli, A. Genome-wide fitness and genetic interactions determined by Tn-seq, a high-throughput massively parallel sequencing method for microorganisms. Curr. Protoc. Microbiol. 36, $1 \mathrm{E}-3$ (2017).

168. Johnson, C. M. \& Grossman, A. D. Identification of host genes that affect acquisition of an integrative and conjugative element in Bacillus subtilis. Mol. Microbiol. (2014) doi:10.1111/mmi.12736.

169. Bolger, A. M., Lohse, M. \& Usadel, B. Trimmomatic: a flexible trimmer for Illumina sequence data. Bioinformatics 30, 2114-2120 (2014).

170. Martin, M. Cutadapt removes adapter sequences from high-throughput sequencing reads. EMBnet. J. 17, 10-12 (2011).

171. Langmead, B. \& Salzberg, S. L. Fast gapped-read alignment with Bowtie 2. Nat. Methods 9, 357 (2012).

172. Koren, S. et al. Canu: scalable and accurate long-read assembly via adaptive k-mer weighting and repeat separation. Genome Res. 27, 722-736 (2017).

173. Kolmogorov, M., Yuan, J., Lin, Y. \& Pevzner, P. A. Assembly of long, error-prone reads using repeat graphs. Nat. Biotechnol. 37, 540-546 (2019).

174. Kurtz, S. et al. Versatile and open software for comparing large genomes. Genome Biol. 5, 12 (2004).

175. Anagnostopoulos, C. \& Spizizen, J. Requirements for transformation in Bacillus subtilis. J. Bacteriol. 81, 741 (1961).

176. Murray, H. \& Koh, A. Multiple regulatory systems coordinate DNA replication with cell growth in Bacillus subtilis. PLoS Genet. 10, (2014).

177. Koo, B.-M. et al. Construction and analysis of two genome-scale deletion libraries for Bacillus subtilis. Cell Syst. 4, 291-305 (2017).

178. Silhavy, T. J., Kahne, D. \& Walker, S. The bacterial cell envelope. Cold Spring Harb. Perspect. Biol. 2, a000414 (2010).

179. Vollmer, W., Blanot, D. \& De Pedro, M. A. Peptidoglycan structure and architecture. FEMS Microbiol. Rev. 32, 149-167 (2008).

180. Egan, A. J. F., Errington, J. \& Vollmer, W. Regulation of peptidoglycan synthesis and remodelling. Nat. Rev. Microbiol. 1-15 (2020).

181. Tsugita, A., Inouye, M., Terzaghi, E. \& Streisinger, G. Purification of bacteriophage T4 lysozyme. J. Biol. Chem. 243, 391-397 (1968).

182. Yokogawa, K., Kawata, S., Nishimura, S., Ikeda, Y. \& Yoshimura, Y. Mutanolysin, bacteriolytic agent for cariogenic streptococci: partial purification and properties. Antimicrob. Agents Chemother. 6, 156-165 (1974).

183. Felch, J. W., Inagami, T. \& Hash, J. H. The N, O-diacetylmuramidase of Chalaropsis species. V. The complete amino acid sequence. J. Biol. Chem. 250, 3713-3720 (1975).

184. Callewaert, L. \& Michiels, C. W. Lysozymes in the animal kingdom. J. Biosci. 35, 127-160 (2010).

185. Thammasirirak, S. et al. Antimicrobial peptides derived from goose egg white lysozyme. Comp. Biochem. Physiol. Part C Toxicol. Pharmacol. 151, 84-91 (2010).

186. Laible, N. J. \& Germaine, G. R. Bactericidal activity of human lysozyme, muramidase-inactive lysozyme, and cationic polypeptides against Streptococcus sanguis and Streptococcus faecalis: inhibition by chitin oligosaccharides. Infect. Immun. 48, 720-728 (1985).

187. Helmann, J. D. The extracytoplasmic function (ECF) sigma factors. Adv. Microb. Physiol. 46, 48-111 (2002).

188. Stringlis, I. A. et al. MYB72-dependent coumarin exudation shapes root microbiome assembly to promote plant health. Proc. Natl. Acad. Sci. 115, E5213-E5222 (2018).

189. te Winkel, J. D., Gray, D. A., Seistrup, K. H., Hamoen, L. W. \& Strahl, H. Analysis of antimicrobialtriggered membrane depolarization using voltage sensitive dyes. Front. cell Dev. Biol. 4, 29 (2016). 
190. Koeppe, R. E. \& Anderson, O. S. Engineering the gramicidin channel. Annu. Rev. Biophys. Biomol. Struct. 25, 231-258 (1996).

191. Bera, A., Biswas, R., Herbert, S. \& Götz, F. The presence of peptidoglycan O-acetyltransferase in various staphylococcal species correlates with lysozyme resistance and pathogenicity. Infect. Immun. 74, 4598-4604 (2006).

192. Hash, J. H. Purification and properties of staphylolytic enzymes from Chalaropsis sp. Arch. Biochem. Biophys. 102, 379-388 (1963).

193. Linthorst, H. J. M. \& Van Loon, L. C. Pathogenesis-related proteins of plants. CRC. Crit. Rev. Plant Sci. 10, 123-150 (1991).

194. Boller, T. Antimicrobial functions of the plant hydrolases, chitinase and B-1, 3-glucanase. in Mechanisms of plant defense responses 391-400 (Springer, 1993).

195. Grover, A. Plant chitinases: genetic diversity and physiological roles. CRC. Crit. Rev. Plant Sci. 31, 5773 (2012).

196. Meier, S. et al. Co-expression and promoter content analyses assign a role in biotic and abiotic stress responses to plant natriuretic peptides. BMC Plant Biol. 8, 24 (2008).

197. Liu, X. et al. Host-induced bacterial cell wall decomposition mediates pattern-triggered immunity in Arabidopsis. Elife 3, e01990 (2014).

198. Brunner, F., Stintzi, A., Fritig, B. \& Legrand, M. Substrate specificities of tobacco chitinases. Plant J. 14, 225-234 (1998).

199. van Scheltinga, A. C. T., Kalk, K. H., Beintema, J. J. \& Dijkstra, B. W. Crystal structures of hevamine, a plant defence protein with chitinase and lysozyme activity, and its complex with an inhibitor. Structure 2,1181-1189 (1994).

200. Trudel, J. \& Asselin, A. Detection of chitinase activity after polyacrylamide gel electrophoresis. Anal. Biochem. 178, 362-366 (1989).

201. Berger, L. R. \& Weiser, R. S. The $\beta$-glucosaminidase activity of egg-white lysozyme. Biochim. Biophys. Acta 26, 517-521 (1957).

202. Neuberger, A. \& Wilson, B. M. Inhibition of lysozyme by N-acyl-D-glucosamine derivatives. Nature 215, 524-525 (1967).

203. Thomas, E. W., McKelvy, J. F. \& Sharon, N. Specific and irreversible inhibition of lysozyme by 2', 3'-epoxypropyl $\beta$-glycosides of $N$-acetyl-D-glucosamine oligomers. Nature 222, 485-486 (1969).

204. Bjurulf, C. \& Wadsö, I. Thermochemistry of Lysozyme-Inhibitor Binding. Eur. J. Biochem. 31, 95-102 (1972).

205. Gottig, N. et al. Xanthomonas axonopodis pv. citri uses a plant natriuretic peptide-like protein to modify host homeostasis. Proc. Natl. Acad. Sci. 105, 18631-18636 (2008).

206. Morse, M., Pironcheva, G. \& Gehring, C. AtPNP-A is a systemically mobile natriuretic peptide immunoanalogue with a role in Arabidopsis thaliana cell volume regulation. FEBS Lett. 556, 99-103 (2004).

207. Matthews, B. W., Grütter, M. G., Anderson, W. F. \& Remington, S. J. Common precursor of lysozymes of hen egg-white and bacteriophage T4. Nature 290, 334-335 (1981).

208. Grütter, M. G., Weaver, L. H. \& Matthews, B. W. Goose lysozyme structure: an evolutionary link between hen and bacteriophage lysozymes? Nature 303, 828-831 (1983).

209. Wang, W. et al. PcMuORP1, an oxathiapiprolin-resistance gene, functions as a novel selection marker for Phytophthora transformation and CRISPR/Cas9 mediated genome editing. Front. Microbiol. 10, 2402 (2019).

210. Leisen, T. et al. CRISPR/Cas with ribonucleoprotein complexes and transiently selected telomere vectors allows highly efficient marker-free and multiple genome editing in Botrytis cinerea. bioRxiv (2020).

211. Klosterman, S. J. et al. Comparative genomics yields insights into niche adaptation of plant vascular wilt pathogens. PLoS Pathog. 7, (2011).

212. Schnathorst, W. C. Life cycle and epidemiology of Verticillium. Fungal wilt Dis. plants 82, (1981). 
213. Bahram, M. et al. Structure and function of the global topsoil microbiome. Nature 560, 233-237 (2018).

214. Giraldo, M. C. \& Valent, B. Filamentous plant pathogen effectors in action. Nat. Rev. Microbiol. 11, 800-814 (2013).

215. Lo Presti, L. et al. Fungal effectors and plant susceptibility. Annu. Rev. Plant Biol. 66, 513-545 (2015).

216. de Oliveira Dias, R. \& Franco, O. L. Cysteine-stabilized $a \beta$ defensins: from a common fold to antibacterial activity. Peptides 72, 64-72 (2015).

217. Hubert, F., Noël, T. \& Roch, P. A member of the arthropod defensin family from edible Mediterranean mussels (Mytilus galloprovincialis). Eur. J. Biochem. 240, 302-306 (1996).

218. Bontems, F., Roumestand, C., Gilquin, B., Menez, A. \& Toma, F. Refined structure of charybdotoxin: common motifs in scorpion toxins and insect defensins. Science. 254, 1521-1523 (1991).

219. Schneider, T. et al. Plectasin, a fungal defensin, targets the bacterial cell wall precursor Lipid II. Science. 328, 1168-1172 (2010).

220. Duressa, D. et al. RNA-seq analyses of gene expression in the microsclerotia of Verticillium dahliae. BMC Genomics 14, 607 (2013).

221. Klimes, A. \& Dobinson, K. F. A hydrophobin gene, VDH1, is involved in microsclerotial development and spore viability in the plant pathogen Verticillium dahliae. Fungal Genet. Biol. 43, 283-294 (2006).

222. Smith, H. C. The morphology of Verticillium albo-atrum, V. dahliae, and V. tricorpus. New Zeal. J. Agric. Res. 8, 450-478 (1965).

223. Krishnan, H. B., Natarajan, S. S., Bennett, J. O. \& Sicher, R. C. Protein and metabolite composition of xylem sap from field-grown soybeans (Glycine max). Planta 233, 921-931 (2011).

224. Yadeta, K. \& Thomma, B. P. H. J. The xylem as battleground for plant hosts and vascular wilt pathogens. Front. Plant Sci. 4, 97 (2013).

225. Pegg, G. F. \& Brady, B. L. Verticillium wilts. (CABI, 2002).

226. Bell, A. A. \& Wheeler, M. H. Biosynthesis and functions of fungal melanins. Annu. Rev. Phytopathol. 24, 411-451 (1986).

227. Caldwell, J. E. et al. Solution structure of the thermostable sweet-tasting protein brazzein. Nat. Struct. Biol. 5, 427-431 (1998).

228. Zhou, L., Zhao, J., Guo, W. \& Zhang, T. Functional analysis of autophagy genes via Agrobacteriummediated transformation in the vascular wilt fungus Verticillium dahliae. J. Genet. genomics 40, 421431 (2013).

229. Song, Y. et al. Transfer of tomato immune receptor Ve1 confers Ave1-dependent Verticillium resistance in tobacco and cotton. Plant Biotechnol. J. 16, 638-648 (2018).

230. Kwak, M.-J. et al. Rhizosphere microbiome structure alters to enable wilt resistance in tomato. Nat. Biotechnol. 36, 1100-1109 (2018).

231. Chen, Y. et al. Wheat microbiome bacteria can reduce virulence of a plant pathogenic fungus by altering histone acetylation. Nat. Commun. 9, 1-14 (2018).

232. Chapelle, E., Mendes, R., Bakker, P. A. H. M. \& Raaijmakers, J. M. Fungal invasion of the rhizosphere microbiome. ISME J. 10, 265-268 (2016).

233. Klein, E., Ofek, M., Katan, J., Minz, D. \& Gamliel, A. Soil suppressiveness to Fusarium disease: shifts in root microbiome associated with reduction of pathogen root colonization. Phytopathology 103, 23-33 (2013).

234. Sarkar, D. et al. The inconspicuous gatekeeper: endophytic Serendipita vermifera acts as extended plant protection barrier in the rhizosphere. New Phytol. 224, 886-901 (2019).

235. Kim, Y. C. et al. The multifactorial basis for plant health promotion by plant-associated bacteria. Appl. Environ. Microbiol. 77, 1548-1555 (2011).

236. Stone, B. W. G., Weingarten, E. A. \& Jackson, C. R. The role of the phyllosphere microbiome in plant health and function. Annu. Plant Rev. online 533-556 (2018).

237. Ritpitakphong, U. et al. The microbiome of the leaf surface of Arabidopsis protects against a fungal pathogen. New Phytol. 210, 1033-1043 (2016). 
238. Berg, M. \& Koskella, B. Nutrient-and dose-dependent microbiome-mediated protection against a plant pathogen. Curr. Biol. 28, 2487-2492 (2018).

239. Spanu, P. D. The genomics of obligate (and nonobligate) biotrophs. Annu. Rev. Phytopathol. 50, 91109 (2012).

240. Hunter, P. J., Hand, P., Pink, D., Whipps, J. M. \& Bending, G. D. Both leaf properties and microbemicrobe interactions influence within-species variation in bacterial population diversity and structure in the lettuce (Lactuca species) phyllosphere. Appl. Environ. Microbiol. 76, 8117-8125 (2010).

241. Mesarich, C. H. et al. Specific hypersensitive response-associated recognition of new apoplastic effectors from Cladosporium fulvum in wild tomato. Mol. plant-microbe Interact. 31, 145-162 (2018).

242. Alfano, J. R. Roadmap for future research on plant pathogen effectors. Mol. Plant Pathol. 10, 805813 (2009).

243. Gibriel, H. A. Y., Thomma, B. P. H. J. \& Seidl, M. F. The age of effectors: genome-based discovery and applications. Phytopathology 106, 1206-1212 (2016).

244. Sperschneider, J., Dodds, P. N., Gardiner, D. M., Singh, K. B. \& Taylor, J. M. Improved prediction of fungal effector proteins from secretomes with EffectorP 2.0. Mol. Plant Pathol. 19, 2094-2110 (2018).

245. Partida-Martinez, L. P. \& Hertweck, C. Pathogenic fungus harbours endosymbiotic bacteria for toxin production. Nature 437, 884-888 (2005).

246. Arendt, K. R., Hockett, K. L., Araldi-Brondolo, S. J., Baltrus, D. A. \& Arnold, A. E. Isolation of endohyphal bacteria from foliar Ascomycota and in vitro establishment of their symbiotic associations. Appl. Environ. Microbiol. 82, 2943-2949 (2016).

247. Deveau, A. et al. Bacterial-fungal interactions: ecology, mechanisms and challenges. FEMS Microbiol. Rev. 42, 335-352 (2018).

248. Ghodsalavi, B. et al. A novel baiting microcosm approach used to identify the bacterial community associated with Penicillium bilaii hyphae in soil. PLoS One 12, e0187116 (2017).

249. Schulz-Bohm, K. et al. Fungus-associated bacteriome in charge of their host behavior. Fungal Genet. Biol. 102, 38-48 (2017).

250. Warmink, J. A., Nazir, R. \& Van Elsas, J. D. Universal and species-specific bacterial 'fungiphiles' in the mycospheres of different basidiomycetous fungi. Environ. Microbiol. 11, 300-312 (2009).

251. Stopnisek, N. et al. Molecular mechanisms underlying the close association between soil Burkholderia and fungi. ISME J. 10, 253-264 (2016).

252. Scheublin, T. R., Sanders, I. R., Keel, C. \& Van Der Meer, J. R. Characterisation of microbial communities colonising the hyphal surfaces of arbuscular mycorrhizal fungi. ISME J. 4, 752-763 (2010).

253. Filion, M., St-Arnaud, M. \& Fortin, J. A. Direct interaction between the arbuscular mycorrhizal fungus Glomus intraradices and different rhizosphere microorganisms. New Phytol. 141, 525-533 (1999).

254. Toljander, J. F., Lindahl, B. D., Paul, L. R., Elfstrand, M. \& Finlay, R. D. Influence of arbuscular mycorrhizal mycelial exudates on soil bacterial growth and community structure. FEMS Microbiol. Ecol. 61, 295304 (2007).

255. Nazir, R., Tazetdinova, D. I. \& van Elsas, J. D. Burkholderia terrae BS001 migrates proficiently with diverse fungal hosts through soil and provides protection from antifungal agents. Front. Microbiol. 5 , 598 (2014).

256. Frey-Klett, P., Garbaye, J. and \& Tarkka, M. The mycorrhiza helper bacteria revisited. New Phytol. 176, 22-36 (2007).

257. Founoune, H. et al. Mycorrhiza helper bacteria stimulate ectomycorrhizal symbiosis of Acacia holosericea with Pisolithus alba. New Phytol. 81-89 (2002).

258. Deveau, A. \& Labbé, J. Mycorrhiza helper bacteria. Mol. mycorrhizal symbiosis 437-440 (2017).

259. Kohlmeier, S. et al. Taking the fungal highway: mobilization of pollutant-degrading bacteria by fungi. Environ. Sci. Technol. 39, 4640-4646 (2005).

260. Simon, A. et al. Exploiting the fungal highway: development of a novel tool for the in situ isolation of bacteria migrating along fungal mycelium. FEMS Microbiol. Ecol. 91, fiv116 (2015). 
261. Nazir, R., Zhang, M., de Boer, W. \& van Elsas, J. D. The capacity to comigrate with Lyophyllum sp. strain Karsten through different soils is spread among several phylogenetic groups within the genus Burkholderia. Soil Biol. Biochem. 50, 221-233 (2012).

262. Furuno, S., Remer, R., Chatzinotas, A., Harms, H. \& Wick, L. Y. Use of mycelia as paths for the isolation of contaminant-degrading bacteria from soil. Microb. Biotechnol. 5, 142-148 (2012).

263. Lackner, G., Moebius, N., Partida-Martinez, L. \& Hertweck, C. Complete genome sequence of Burkholderia rhizoxinica, an endosymbiont of Rhizopus microsporus. J. Bacteriol. 193, 783-784 (2011).

264. Ahn, I.-P. \& Lee, Y.-H. A viral double-stranded RNA up regulates the fungal virulence of Nectria radicicola. Mol. Plant-Microbe Interact. 14, 496-507 (2001).

265. Okada, R. et al. Molecular characterization of a novel mycovirus in Alternaria alternata manifesting two-sided effects: Down-regulation of host growth and up-regulation of host plant pathogenicity. Virology 519, 23-32 (2018).

266. Delves-Broughton, J., Blackburn, P., Evans, R. J. \& Hugenholtz, J. Applications of the bacteriocin, nisin. Antonie Van Leeuwenhoek 69, 193-202 (1996). 



\section{Summary}

To facilitate disease establishment, plant pathogenic microbes secrete a wide diversity of effectors that promote host colonization through a multitude of mechanisms. Typically, effectors are considered to be small cysteine-rich in planta-secreted proteins, most of which are thought to be involved in the deregulation of host immune responses or in the manipulation of other aspects of host physiology. Consequently, effector proteins are almost exclusively studied in the context of binary plant-pathogen interactions. However, plants associate with numerous microbes that collectively form their microbiota. It is becoming increasingly evident that plant microbiomes, i.e. the microbes and their genomes in their environment, are an important determinant for plant health. Moreover, plants actively shape their microbiome compositions to suppress potential pathogens.

In Chapter 1 we hypothesize that microbial plant pathogens manipulate plant microbiomes through the secretion of particular effector proteins with antimicrobial activity to promote disease establishment on their hosts. Furthermore, the organism that was studied to address this hypothesis, namely the soil-borne broad host-range fungal plant pathogen Verticillium dahliae, is introduced.

Chapter 2 provides an opinion manuscript in which we elaborate on the hypothesis that plant pathogens secrete effector proteins to manipulate host microbiomes. Additionally, we propose a number of strategies that can be exploited to identify such effector proteins.

In Chapter $\mathbf{3}$ we show that the previously identified $V$. dahliae virulence effector VdAve1 is a protein with selective antibacterial activity that facilitates colonization of tomato and cotton through the manipulation of their microbiomes by suppressing bacteria with antagonistic activity towards $V$. dahliae. Moreover, we show that VdAve1, and also the newly identified antimicrobial effector VdAMP2, are exploited for microbiome manipulation in the soil, where the fungus resides in absence of a host. Thus, we provide evidence for the hypothesis that fungal plant pathogens utilize effector proteins to modulate microbiome compositions inside and outside the host, and propose that pathogen effector catalogs represent an untapped resource for novel antibiotics.

In vitro antimicrobial activity assays uncovered that $\mathrm{VdAve} 1$ inhibits growth of various bacterial species, including the Gram-positive bacterium Bacillus subtilis. By subjecting $B$. subtilis to transcriptome profiling and forward genetic analyses, we reveal in Chapter 4 that similar processes operate in $B$. subtilis in response to VdAve1 as in defense against lysozyme. Furthermore, we show that teichoic acids play a prominent role in tolerance against the detrimental activity of the VdAve1 effector protein. Collectively, the data in this chapter suggest that $\mathrm{VdAve} 1$ may act as a lysozyme. 
Lysozymes are antimicrobial enzymes that target the bacterial cell wall polymer peptidoglycan by hydrolyzing the $\beta-1,4$ glycosidic bonds between the $N$-acetylmuramic acid and $N$-acetylglucosamine subunits. Although lysozymes are ubiquitous in a diversity of organisms, as they are found in animals and in viruses, they are extremely rare in fungi and have not been described in plants. VdAve1 shares no homology with known hydrolytic enzymes and is not predicted to carry any enzymatic domain. In Chapter 5, we show that VdAve1 is able to hydrolyze peptidoglycan, thereby uncovering the effector as a novel type of lysozyme. In addition to its hydrolytic activity, we show that $\mathrm{VdAve1}$, like many previously described lysozymes, also exerts non-enzymatic antimicrobial activity that involves direct cell membrane perturbation, which is mediated by an arginine- and lysine-rich peptide that is embedded within the protein. Likely, these two activities complement each other as the peptidoglycan hydrolase activity of VdAve 1 is likely to facilitate the access of the cationic peptide to the bacterial cell membrane. Importantly, V. dahliae originally acquired VdAve 1 through horizontal gene transfer from plants, where $V d A v e 1$ homologs are ubiquitous and annotated as plant natriuretic peptides (PNPs), several members of which have been characterized as stress-related proteins with homeostatic roles in the regulation of ion fluxes, stomatal movement and fluid circulation affecting plant biological activities such as photosynthesis and respiration. Based on our findings, we propose that these PNPs are the plant lysozymes that have remained enigmatic thus far.

Following systemic host colonization, $V$. dahliae produces multicellular melanized resting structures, called microsclerotia, in the decaying tissues of its hosts. After host tissue decomposition, these resting structures are released into the soil where the pathogen can survive for many years. In Chapter $\mathbf{6}$ we describe the identification and characterization of the defensin-like $V$. dahliae effector protein VdAMP3. We show that VdAMP3 has antimicrobial activity and that VdAMP3 is specifically expressed in hyphal sections that develop into microsclerotia, suggesting that $V$. dahliae exploits VdAMP3 to protect microsclerotia formation. Accordingly, we show that VdAMP3 contributes to $V$. dahliae biomass accumulation in decaying host tissue. Hence, our findings demonstrate that $V$. dahliae employs VdAMP3 to protect its microsclerotia and corroborate the hypothesis that $V$. dahliae exploits different antimicrobial effector proteins at different stages of its life cycle.

Finally, Chapter $\mathbf{7}$ discusses the results obtained in this thesis and provides an outlook for the anticipated roles of fungal plant pathogen effector proteins in microbiome manipulation in a broader context. Moreover, potential implications and applications of our finding that plant pathogens exploit effectors for microbiome manipulation are discussed with respect to plant disease control and the development of novel antibiotics. 


\section{Acknowledgements}

It's done, my PhD thesis has been completed! It still feels like yesterday that I joined the Laboratory of Phytopathology for my MSc thesis, but in reality already five years have passed and the MSc thesis only proved to be a stepping stone towards the start of a $\mathrm{PhD}$ trajectory. Along these years I have got to enjoy tremendous support from many people, without whom I would not have been able to put together this booklet. Here, I would like to take the opportunity to express my gratitude to these people.

First and foremost I would like to thank my supervisor. Bart, I am not quite sure what words do justice to how grateful I am for your excellent guidance. You very well understand what it takes to make me feel comfortable in a PhD trajectory full with challenges. Without your skills as a true people manager this booklet would never have been completed! Thanks for all your patience and for the times you helped me back on my feet after I thought I hit rock-bottom once again. Finally, thank you so much for your trust in my abilities and for the corresponding freedom that you offered me to perform my research, I could not be more excited to continue this work as part of your renewed team!

I would like to continue by thanking two people that played an important role mostly, but not exclusively, during my MSc theses and who through their input and supervision positively impacted my $\mathrm{PhD}$ project at length. Hanna, thanks for providing me a crash course effector biology at Phyto. The diversity of techniques that we covered in a relatively short period was invaluable to kickstart my own projects. Your professionalism has always continued to serve as an example to me. It frustrates me that we have only completed part of the work on VdAve1 thus far, but I am confident we will fix this in Cologne. Jeroen, thank you for welcoming me in Lübeck and for your invaluable input on the purification of $\mathrm{VdAve1}$. Although we did not yet realize it at that time, your definition of VdAve1 as "the killer protein" proved to be spot on in retrospect.

Next, I would like to thank two outstanding students I was privileged to supervise during my PhD; Mercedes and Gabriella. Merce, it did not take long for me to realize that you were a very talented student. Your eagerness to learn combined with your uninhibited attitude when performing your experiments greatly accelerated the progress of our studies. The findings described in your MSc thesis have provided me a very solid basis to build on. Gabriella, it took you a while before you decided to join me for your thesis, but I am so glad you eventually did. Although you tried really hard to downplay the expectations by convincing me you were likely to screw up your project, it quickly became obvious that you were going to do a great job. Indeed, not quite unexpected, your hard work resulted in multiple exciting discoveries, most of which have been included in this thesis. Merce and Gabriella, thanks for sharing your contagious enthusiasm with me, the synergy I experienced during the time I got to collaborate with each of you I consider the highlight of my time as a $\mathrm{PhD}$ candidate. 
In addition to the people mentioned above, many others have made significant contributions to the work presented in this booklet. Michael, thanks for helping me out with all my bioinformatics related questions and especially for your support on the $16 \mathrm{~s}$ profiling analyses. I look forward to an intensified collaboration! Grardy, thank you for keeping the Verticillium lab running at all times, and certainly also for your recent efforts that contributed to the completion of Chapter 3. Gabriel, thanks for all those times we teamed up to streamline our protein purifications, V. dahliae transformations, xylem fluid isolations and many other things. It is always a pleasure working with you! Edgar, thank you for introducing me to the world of Nanopore sequencing, I very much enjoyed sequencing the $B$. subtilis isolates with you. Bert, I am so grateful for your craftsmanship! Good plant care is invaluable for $V$. dahliae disease assays, and I am pretty sure that if it wasn't for you, I was still performing experiments in Unifarm at this moment. Reindert, rarely a single week passed during which I did not use any of your bacterial endophytes, so thanks a lot for sharing them with me. Also many thanks for putting us in contact with Leendert, it proved to be a gamechanger for the characterization of VdAve1. Leendert, you and your team have provided me with a warm welcome at the UvA, which I am really grateful for. It was nice to experience what a bacteriology lab is like. Biwen, I appreciate your open attitude and I very much enjoyed our shared project. Your detailed and accurate protocols for the RNA-seq and Tn-seq library preparation made my life very easy. I'm glad our collaborative efforts have paid off! Finally, Tjalling, thanks for your technical assistance on the fluorescence microscopy as well as the DiSC assay.

I also would like to acknowledge everyone who provided me with valuable input during work discussions, seminars or coffee breaks. I especially would like to thank Jan, David, Mireille, Luis and Malaika for the many times they provided me food for thought. Damn I miss our brainstorm sessions in the lab, Malaika! Too bad we only got to be lab buddies for two years because there was plenty of Friday afternoon experiments left to run on "Roji". Also many thanks to all our former and present staff members. I especially would like to express my gratitude to Grardy, Ali, Ester, Laurens and Gert for their efforts that secured the daily routine in the lab. Additionally, I want to thank all fellow PhDs and Postdocs at Phyto for any kind of support and for the fun moments we have shared; special thanks to Nelia, David, Xiaoqian, Jasper V., Jasper D., Sander, Laura, Maikel and Si.

Along the course of my PhD many people have come and gone. However, I was also privileged to share the whole trajectory with some great fellow students. Katharina, the simultaneous completion of our PhDs marks the end of a six-year companionship at Wageningen University. I am really grateful for the friendship we have built over these years, and I cannot thank you enough for all your support! I am flattered you asked me to be your paranymph and I cannot wait to see you rock the stage in December. Martin, I believe our personalities differ just as much as Friesland and Brabant are 
apart, for Dutch standards. I nevertheless very much enjoyed the time we have shared at Phyto, initially next to each other in the lab, and later together with Hui in the writing office. I will never forget when the three of us went to visit Malaika in Cologne and that (after a couple of beers) you decided to present my work discussion for us. I have to say, you did a pretty good job! Hui, you are one of the kindest persons I know! Thank you for flooding our laboratory with happiness and good vibes, with you around it was always a lovely place to work. You are always willing to help, Lotte and I could even call you when we were stuck on the Chinese countryside and when our nonverbal communication proved insufficient to express ourselves to the locals. Finally, many thanks for all those times you have spoiled me and other colleagues with your dinners. Even after having tasted some amazing food in China, I still believe the best Chinese food is served at your place.

I would like to conclude by acknowledging the most important people in my life.

Britt, my lovely sister, I realize "all this science stuff" is not your cup of tea, but you nevertheless always expressed your sincere appreciation for the career path I have chosen. Knowing that my little sister is proud of her big brother means the world to me. I hope you have finally come to realize that science is not only for geeks. Science is quite fun you know, and so are scientists, I would definitely consider dating one if I were you.

Pap and mam, thank you so much for your unconditional support! You have always offered Britt and me such a loving home. For me the Schepenstraat still represents a place to which I can come home to from time to time, for instance to put my mind at ease during times of stress. More than once, a short weekend visit to Bladel was inevitable for me to find the courage to continue. The fact that you will always have my back makes everything just so much easier for me, thanks for that!

Finally, Lotte, sweet sweet Lotte, I am so happy to have you by my side! More than anyone you have seen (or should I say experienced?) how much I had to fight my insecurities to get this job done. Luckily, you were always there to pull me through and to reassure me I could do it. Life is so much fun with you! Although it is the thing I enjoy doing most, it does not necessarily take a trip around the world to have a great time with you, we even managed to turn my first paper rejection into a celebration. Soon you will compile your own $\mathrm{PhD}$ thesis and that for sure will become a beautiful booklet, which makes me super proud. You can do it! I love you! 


\section{About the author}

Nick C. Snelders was born in Bladel, The Netherlands, on June $24^{\text {th }} 1992$. Following the completion of the Atheneum at the Pius X-College in Bladel, in 2010 he joined Fontys Hogenscholen in Eindhoven for his BSc studies in Applied Science where he specialized in molecular biology. In 2012, he joined the laboratory of Prof. Christopher Franco at Flinders University in Adelaide, Australia, for his BSc thesis on the biological control of soil-borne potato pathogens using Streptomyces spp.. During this period Nick

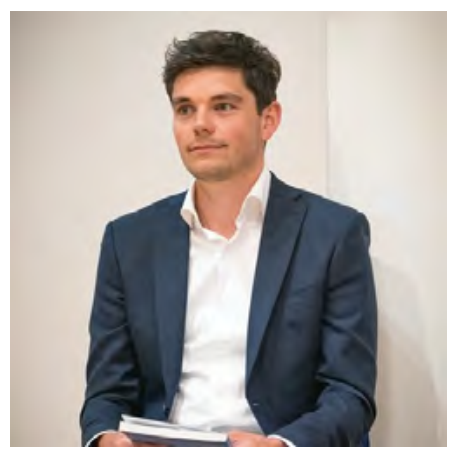
got inspired by plant sciences and phytopathology, which eventually drove him to Wageningen University in 2014 for his MSc studies on plant biotechnology. During his second year in Wageningen, Nick joined the group of Prof. Bart Thomma for his MSc thesis to study the effector protein VdAve1 that is secreted by the soil-borne fungal plant pathogen Verticillium dahliae. Following the completion of his thesis, Nick was offered a PhD position in Prof. Bart Thomma's group, which he gratefully accepted. Prior to the initiation of his PhD project, Nick joined Dr. Jeroen Mesters at the Department of Biochemistry at the University of Lübeck, Germany, with whom he optimized the heterologous production and purification of VdAve1, to facilitate the functional characterization of this effector protein. In September 2016, Nick rejoined the group of Prof. Bart Thomma at the Laboratory of Phytopathology in Wageningen as a PhD candidate to study how $V$. dahliae exploits effector proteins, including VdAve1, to promote niche colonization through the manipulation of microbiomes. Since October 2020, Nick is a postdoctoral researcher in the newly established Evolutionary Microbiology Lab of Prof. Bart Thomma at the University of Cologne in a collaborative project with Dr. Michael Seidl at the Theoretical Biology \& Bioinformatics department of Utrecht University. 


\section{List of publications}

Snelders N.C.\#, Kettles G.J.\#, Rudd J.J., Thomma B.P.H.J. Plant pathogen effector proteins as manipulators of host microbiomes? Mol. Plant Pathol. 19, 257 (2018)

Snelders N.C., Rovenich H.\#, Petti G.C.\#, Rocafort M., van den Berg G.C.M., Vorholt J.A., Mesters J.R., Seidl M.F., Nijland R., Thomma B.P.H.J. Microbiome manipulation by a soil-borne fungal plant pathogen using effector proteins. Nature Plants (2020) in press.

Snelders N.C., Wang B., Chavarro-Carrero E.A., Siersma T., Hamoen L.W.\#, Thomma B.P.H.J." Teichoic acids protect Bacillus subtilis against the antimicrobial Verticillium dahliae effector VdAve1. In preparation.

Snelders N.C., Siersma T., Hamoen L.W., Thomma B.P.H.J. Functional characterization uncovers the antimicrobial Verticillium dahliae effector VdAve1 as a novel type of lysozyme. In preparation.

Snelders N.C., Petti G.C., Thomma B.P.H.J. A Verticillium dahliae defensin-like effector protein promotes microsclerotia formation and survival after host decay. In preparation.

\# Equal contribution 

The research described in this thesis was financially supported by the Research Council Earth and Life Sciences (ALW) of the Netherlands Organization of Scientific Research (NWO). Financial support from Wageningen University for printing this thesis is gratefully acknowledged.

Cover \& layout design: Iliana Boshoven-Gkini | AgileColor.com Printed by GVO drukkers \& vormgevers B.V. | gvo.nl 
